WSRC-TR-2001-00559, Rev. 1

\title{
Feed Basis for Processing Relatively Low Radioactivity Waste Tanks
}

J. A. Pike

M. D. Drumm

S. G. Subosits

J. L. Statton

F. A. Washburn

Westinghouse Savannah River Company

Savannah River Site

Aiken, South Carolina 29808

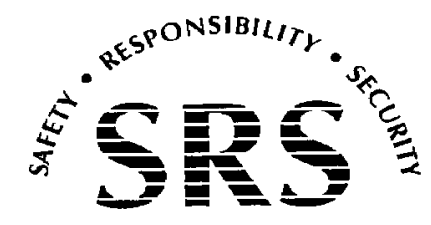


This document was prepared in conjunction with work accomplished under Contract No. DE-AC09-96SR18500 with the U. S. Department of Energy.

\section{DISCLAIMER}

This report was prepared as an account of work sponsored by an agency of the United States Government. Neither the United States Government nor any agency thereof, nor any of their employees, makes any warranty, express or implied, or assumes any legal liability or responsibility for the accuracy, completeness, or usefulness of any information, apparatus, product or process disclosed, or represents that its use would not infringe privately owned rights. Reference herein to any specific commercial product, process or service by trade name, trademark, manufacturer, or otherwise does not necessarily constitute or imply its endorsement, recommendation, or favoring by the United States Government or any agency thereof. The views and opinions of authors expressed herein do not necessarily state or reflect those of the United States Government or any agency thereof.

This report has been reproduced directly from the best available copy.

Available for sale to the public, in paper, from: U.S. Department of Commerce, National Technical Information Service, 5285 Port Royal Road, Springfield, VA 22161, phone: (800) 553-6847, fax: (703) 605-6900

email: orders@ntis.fedworld.gov

online ordering: http://www.ntis.gov/help/index.asp

Available electronically at http://www.osti.gov/bridge

Available for a processing fee to U.S. Department of Energy and its contractors, in paper, from: U.S. Department of Energy, Office of Scientific and Technical Information, P.O. Box 62, Oak Ridge, TN 37831-0062,

phone: (865)576-8401,

fax: (865)576-5728

email: $\underline{\text { reports@ adonis.osti.gov }}$ 
WSRC-TR-2001-00559, Rev. 1 Keywords: Salt Processing, Waste

Characterization

Retention: Permanent

Publication Date: February 25, 2002

\section{Feed Basis for Processing Relatively Low Radioactivity Waste Tanks}

J. A. Pike

M. D. Drumm

S. G. Subosits

J. L. Statton

F. A. Washburn

Westinghouse Savannah River Company

Savannah River Site

Aiken, South Carolina 29808

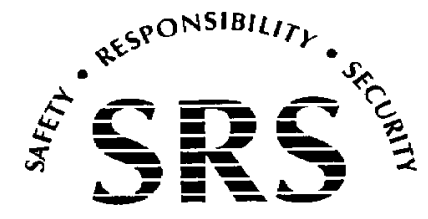


WSRC-TR-2001-00559, Rev. 1

This page intentionally left blank. 


\section{Feed Basis for Processing Relatively Low Radioactivity Waste Tanks}

\section{Authors}

Juph Pche

J. A. Pike, High-Level Waste Process Engineering

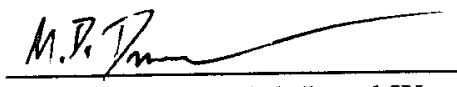

M. D. Drumm, High-Level Waste Process Engineering

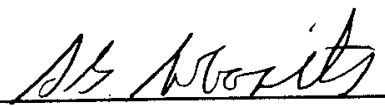

S. G. Subosits, High-Level Waste Process Engineering

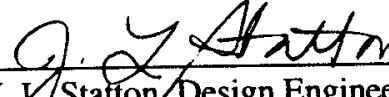

J. Y. Statton, Design Engineering Salt Waste Processing Facility

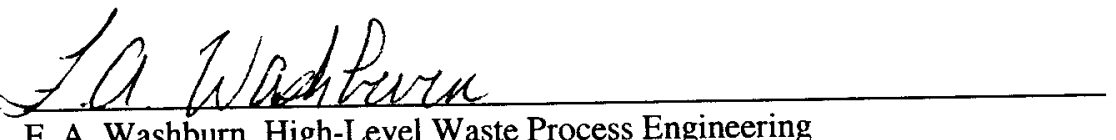

F. A. Washburn, High-Level Waste Process Engineering

\section{Approvals}
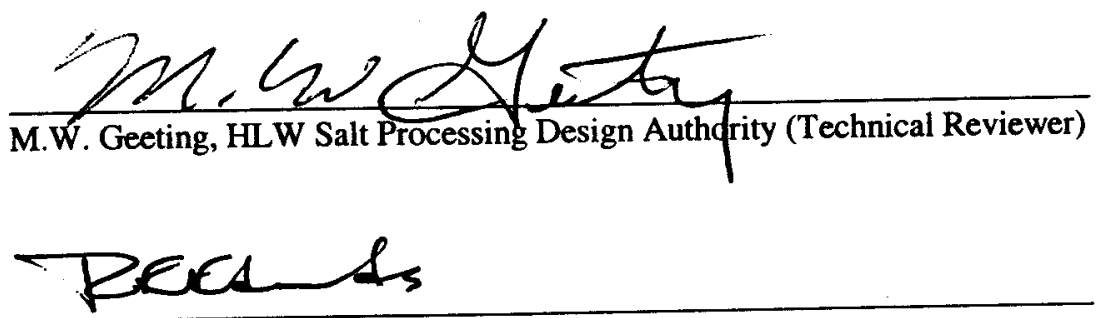

R. E. Edwards, Manager, HLW Processing Engineering $\frac{5 / 7 / 02}{\text { Date }}$

$\frac{4 / 23 / 02}{\text { Date }}$

$\frac{4 / 23 / 02}{\text { Date }}$

$4-23-02$

Date

$4 / 23 / 02$

Date

$\frac{4 / 29 / 02}{\text { Date }}$

$\frac{6 / 10 / 02}{\text { Date }}$ 
WSRC-TR-2001-00559, Rev. 1

\begin{abstract}
This paper presents the characterization of potential feed for processing relatively low radioactive waste tanks. The feed characterization is based on waste characterization data extracted from the waste characterization system. This data is compared to salt cake sample results from Tanks 37, 38 and 41.
\end{abstract}


WSRC-TR-2001-00559, Rev. 1

\section{CONTENTS}

$\begin{array}{lr}\text { Introduction } & 1 \\ \text { Approach } & 1 \\ \text { Salt Cake and Feed Solution Characterization } & 2 \\ \text { Real Waste Salt Cake Samples } & 35 \\ \text { References } & 40 \\ \text { Appendix A } & 41\end{array}$

\section{LIST OF TABLES}

Table 1: Salt Cake Composition 4

Table 2: Supernate Composition $\quad 5$

Table 3: Average Radionuclide Concentration in Sludge 7

Table 4: Projected Feed Composition After Dissolution and Dilution to 6.44 M Total

Sodium Concentration $600 \mathrm{mg} / \mathrm{L}$ sludge; $30 \%$ of interstitial supernate remaining $\quad 8$

Table 5: Projected Feed Composition After Dissolution and Dilution to 6.44 M Total

Sodium Concentration $350 \mathrm{mg} / \mathrm{L}$ sludge; $30 \%$ of interstitial supernate remaining

Table 6: Projected Feed Composition After Dissolution and Dilution to 6.44 M Total

Sodium Concentration $100 \mathrm{mg} / \mathrm{L}$ sludge; $30 \%$ of interstitial supernate remaining

1

Table 7: Projected Feed Composition After Dissolution and Dilution to 6.44 M Total Sodium Concentration no sludge; $30 \%$ of interstitial supernate remaining

Table 8: Projected Feed Composition After Dissolution and Dilution to 6.44 M Total

Sodium Concentration $600 \mathrm{mg} / \mathrm{L}$ sludge; $20 \%$ of interstitial supernate remaining

Table 9: Projected Feed Composition After Dissolution and Dilution to 6.44 M Total

Sodium Concentration $350 \mathrm{mg} / \mathrm{L}$ sludge; $20 \%$ of interstitial supernate remaining

Table 10: Projected Feed Composition After Dissolution and Dilution to 6.44 M Total

Sodium Concentration $100 \mathrm{mg} / \mathrm{L}$ sludge; $20 \%$ of interstitial supernate remaining

Table 11: Projected Feed Composition After Dissolution and Dilution to 6.44 M Total

Sodium Concentration no sludge; $20 \%$ of interstitial supernate remaining

Table 12: Projected Feed Composition After Dissolution and Dilution to 6.44 M Total

Sodium Concentration $600 \mathrm{mg} / \mathrm{L}$ sludge; $10 \%$ of interstitial supernate remaining

Table 13: Projected Feed Composition After Dissolution and Dilution to 6.44 M Total

Sodium Concentration $350 \mathrm{mg} / \mathrm{L}$ sludge; $10 \%$ of interstitial supernate remaining

Table 14: Projected Feed Composition After Dissolution and Dilution to 6.44 M Total

Sodium Concentration $100 \mathrm{mg} / \mathrm{L}$ sludge; $10 \%$ of interstitial supernate remaining

Table 15: Projected Feed Composition After Dissolution and Dilution to 6.44 M Total

Sodium Concentration no sludge ; $10 \%$ of interstitial supernate remaining

Table 16: Projected Feed Volumes After Dissolution and Dilution to 6.44 M Total

Sodium Concentration: $30 \%$ interstitial supernate remaining

Table 17: Projected Feed Volumes After Dissolution and Dilution to 6.44 M Total

Sodium Concentration: $20 \%$ interstitial supernate remaining

Table 18: Projected Feed Volumes After Dissolution and Dilution to 6.44 M Total

Sodium Concentration: $10 \%$ interstitial supernate remaining

Table 19: Comparison of Real Salt Cake Sample Results with Predicted Compositions 
WSRC-TR-2001-00559, Rev. 1

This page intentionally left blank 


\title{
Feed Basis for Processing Relatively Low Radioactivity Waste Tanks
}

\author{
By J. A. Pike, M. D. Drumm, S. G. Subosits, J. L. Statton, and F. A. Washburn
}

\section{Introduction}

To prepare cesium (Cs) for final disposal, it is necessary to separate Cs and other soluble transuranic material from the waste stream. The original baseline salt processing step at SRS, known as In-Tank Precipitation (ITP), initiated operation in September 1995. During operations, benzene (a flammable gas) evolved from the process at higher rates than expected.

In August 1996, the Defense Nuclear Facilities Safety Board (DNFSB) issued Recommendation 96-1 that operations and tests in the ITP Facility not proceed without an improved understanding of the mechanisms of benzene generation, retention, and release. Extensive laboratory studies conducted from December 1995 through January 1998 ultimately concluded that production goals and safety requirements could not be simultaneously met in the ITP Facility, as configured. This resulted in a Westinghouse Savannah River Company (WSRC) recommendation to the Department of Energy in January 1998 to suspend ITP operations and conduct a systems engineering evaluation of salt disposition options and to recommend the preferred alternatives.

DOE designated a preferred alternative, Caustic Side Solvent Extraction, in October 2001.As described in the Salt Processing EIS Record of Decision (ROD), DOE plans to pursue a variety of methods to disposition the salt waste. These are:

- $\quad$ Low Curie Salt Waste Direct to Saltstone (no treatment)

- Actinide Removal from Low Curie Waste followed by Disposal in Saltstone

- Actinide Removal followed by future treatment of the resulting stream rich in Cs

- Caustic Side Solvent Extraction

This paper presents the characterization of potential feed for processing relatively low radioactive waste tanks. The feed characterization is based on waste characterization data extracted from the Waste Characterization System (WCS). This data is compared to salt cake sample results from Tanks 37, 38 and 41. Any revisions to the expected feed will be represented by revisions to this document.

\section{Approach}

Salt cake in High Level Waste (HLW) tanks consists of crystallized salts with interstitial space and entrained insoluble solids assumed to be sludge solids. In order to determine the composition of the salt cake, the individual parts are first identified and then combined in the appropriate ratios to formulate the final characterization. The feed material for processing relatively low radioactivity waste tanks consists of the salt cake dissolved to an assumed 6.44 M total sodium concentration.

The interstitial space is assumed to consist of gas and supernate. Prior to dissolving the salt cake, the interstitial space is assumed to be drained such that less than $30 \%$ liquid supernate remains. Such drainage is possible in a laboratory test on simulated salt cake. ${ }^{1}$ Hydraulic modeling of a full-scale tank indicates the same may be possible at full-scale. ${ }^{2}$ The total interstitial liquid volume is assumed to be $22 \%$. $^{3}$

Chemical and Radionuclide concentrations for salt cake and supernate in each waste tank with salt cake are extracted from the HLW Waste Characterization System (WCS) ${ }^{3}$ or calculated from the information given in the WCS. Tanks with salt cake and no free supernate data are not included. WCS indicates radionuclides are assumed to be in the interstitial supernate and entrained insoluble solids and not in the salt cake solids. 
The radionuclide and chemical data are adjusted to solutions at $6.44 \mathrm{M}$ total sodium concentration by simulating dissolution and dilution with water. The sodium concentration in actual material transferred as feed will change from existing concentrations due to in-transfer dilution.

The radionuclide contribution of the insoluble solids is estimated from the average distribution of radionuclides in HLW sludge, which is extracted from the WCS. The radionuclide contribution range of sludge is then calculated by multiplying the mass-based distribution by $0,100,350$ and $600 \mathrm{mg}$ of sludge solids per liter of final solution. ${ }^{3}$ The sludge contribution is then added to the supernate contribution estimated above.

\section{Salt Cake and Feed Solution Characterization}

Waste tanks with Salt cake include Tanks 1, 2, 3, 9, 10, 25, 27, 28, 29, 31, 33, 34, 36, 37, 38, 41, 44, 45, 46, and 47. Tanks $1,2,3,9$, and 10 do not have transferable free supernate. Without adequate supernate, no supernate sample data exists from the last 15 years to adequately characterize the radionuclide inventory, therefore, these tanks were eliminated from further analysis.

Table 1 shows the salt cake composition as calculated from the information given in the WCS. WCS lists only two compositions for salt cake, one for F-Area salt waste and one for H-Area salt waste, thus, Table 1: Salt Cake Composition shows Tanks in F-Area have the same composition and the tanks in H-Area have the same composition. Table 2 shows the supernate composition per tank extracted from WCS and converted to a consistent set of units. Appendix A contains total gamma data from the Tank Chemistry Database, which provides a comparison of current cesium values in WCS to historical analysis. Table 3 shows the average composition of sludge from WCS. Average sludge composition is estimated by dividing the total of each component by the total mass of sludge in both tank farms as reported by WCS. Table 4 - Table 7 show estimated composition of the feed material produced after draining $70 \%$ of the supernate from the salt cake and with variable amounts of entrained insoluble solids. Table 8 Table 15 shows additional estimates of feed material composition assuming interstitial liquid drainage of $80 \%$ and $90 \%$. All feed compositions are projected by dissolving the salt to $6.44 \mathrm{M}$ total sodium concentration with water.

Tables 16, 17, and 18 show the total estimated volume of salt solution produced after dissolution and dilution of each waste tank with salt cake at 70\%, $80 \%$ and $90 \%$ supernate drainage respectively. To account for the non-ideal solution, the specific gravity of the dissolved salt solution was calculated based on the sodium concentration. ${ }^{8}$ The amount of dilution water required to give a final dissolved salt solution sodium concentration of $6.44 \mathrm{M}$ was then determined algebraically.

$\rho_{\mathrm{ss}}=\left(1.009+.04454 *[\mathrm{Na}]_{\mathrm{ss}}\right) * 8.34$

$\rho_{\mathrm{ss}}=\mathrm{m}_{\mathrm{ss}} / \mathrm{V}_{\mathrm{ss}}$

$\mathrm{m}_{\mathrm{ss}}=\mathrm{m}_{\mathrm{ds}}+\mathrm{m}_{\mathrm{H} 2 \mathrm{O}}$

Combining equations 2 and 3 and equating them to equation 1 , yields

$\left(\mathrm{m}_{\mathrm{ds}}+\mathrm{m}_{\mathrm{H} 2 \mathrm{O}}\right) / \mathrm{V}_{\mathrm{ss}}=\left(1.009+.04454 *[\mathrm{Na}]_{\mathrm{ss}}\right) * 8.34$

$\mathrm{m}_{\mathrm{H} 2 \mathrm{O}}=\mathrm{V}_{\mathrm{ss}} *\left[\left(1.009+.04454 *[\mathrm{Na}]_{\mathrm{ss}}\right) * 8.34\right]-\mathrm{m}_{\mathrm{ds}}$

$\mathrm{V}_{\mathrm{ss}}=[\mathrm{Na}]_{\mathrm{ds}} /[\mathrm{Na}]_{\mathrm{ss}} * \mathrm{~V}_{\mathrm{ds}}$

Inserting equation 6 into equation 5 yields

$\mathrm{m}_{\mathrm{H} 2 \mathrm{O}}=[\mathrm{Na}]_{\mathrm{ds}} /[\mathrm{Na}]_{\mathrm{ss}} * \mathrm{~V}_{\mathrm{ds}} *\left[\left(1.009+.04454 *[\mathrm{Na}]_{\mathrm{ss}}\right) * 8.34\right]-\mathrm{m}_{\mathrm{ds}}$

$\mathrm{V}_{\mathrm{H} 20}=\mathrm{m}_{\mathrm{H} 2 \mathrm{O}} / \rho_{\mathrm{H} 2 \mathrm{O}}$ 
Combining equations 7 and 8 yield

$\mathrm{V}_{\mathrm{H} 2 \mathrm{O}}=\left\{[\mathrm{Na}]_{\mathrm{ds}} /[\mathrm{Na}]_{\mathrm{ss}} * \mathrm{~V}_{\mathrm{ds}} *\left[\left(1.009+.04454 *[\mathrm{Na}]_{\mathrm{ss}}\right) * 8.34\right]-\mathrm{m}_{\mathrm{ds}}\right\} / \rho_{\mathrm{H} 2 \mathrm{O}}$

ss $=$ salt solution after dilution

ds $=$ drained salt

$\mathrm{H} 2 \mathrm{O}=$ dilution water

$\rho=$ density $(\mathrm{lb} / \mathrm{gal})$

$[\mathrm{Na}]=$ sodium concentration $(\mathrm{M})$

$\mathrm{V}=$ volume $(\mathrm{gal})$

$\mathrm{m}=\operatorname{mass}(\mathrm{lb})$ 


\section{Table 1: Salt Cake Composition}

\begin{tabular}{|c|c|c|c|c|c|c|c|c|c|c|c|c|c|c|c|}
\hline \multicolumn{16}{|c|}{ Salt Composition (M) } \\
\hline & Tank 25 & Tank 27 & Tank 28 & Tank 29 & Tank 31 & Tank 33 & Tank 34 & Tank 36 & Tank 37 & Tank 38 & Tank 41 & Tank 44 & Tank 45 & Tank 46 & Tank 47 \\
\hline $\mathrm{Na}$ & $1.90 \mathrm{E}+01$ & $1.90 \mathrm{E}+01$ & $1.90 \mathrm{E}+01$ & $2.06 \mathrm{E}+01$ & $2.06 \mathrm{E}+01$ & $1.90 \mathrm{E}+01$ & $1.90 \mathrm{E}+01$ & $2.06 \mathrm{E}+01$ & $2.06 \mathrm{E}+01$ & $2.06 \mathrm{E}+01$ & $2.06 \mathrm{E}+01$ & $1.90 \mathrm{E}+01$ & $1.90 \mathrm{E}+01$ & $1.90 \mathrm{E}+01$ & $1.90 \mathrm{E}+01$ \\
\hline $\mathrm{Al}(\mathrm{OH}) 4$ & & 9.52E-01 & & $28 \mathrm{E}+00$ & & & & $1.28 \mathrm{E}+00$ & & & & & & & \\
\hline $\mathrm{CO3}$ & & & & & & & & -01 & & & & & & & \\
\hline C2O4 & $6.67 \mathrm{E}-02$ & $6.67 \mathrm{E}-02$ & $6.67 \mathrm{E}-02$ & $4.54 \mathrm{E}-02$ & $4.54 \mathrm{E}-02$ & $6.67 \mathrm{E}-02$ & & $4.54 \mathrm{E}-02$ & $4.54 \mathrm{E}-02$ & $4.54 \mathrm{E}-02$ & $4.54 \mathrm{E}-02$ & $6.67 \mathrm{E}-02$ & $6.67 \mathrm{E}-02$ & $6.67 \mathrm{E}-02$ & $6.67 \mathrm{E}-02$ \\
\hline NO2 & $3.03 \mathrm{E}-01$ & $3.03 \mathrm{E}-01$ & $3.03 \mathrm{E}-01$ & $2.37 \mathrm{E}+00$ & $2.37 \mathrm{E}+00$ & $3.03 \mathrm{E}-01$ & & & & $2.37 \mathrm{E}+00$ & $2.37 \mathrm{E}+00$ & $3.03 \mathrm{E}-01$ & $3.03 \mathrm{E}-01$ & $3.03 \mathrm{E}-01$ & $3.03 \mathrm{E}-01$ \\
\hline & & +01 & +01 & +00 & +00 & & & & & $E+00$ & $E+00$ & +01 & +01 & $E+01$ & +01 \\
\hline & & 2.68 & +00 & $5.91 \mathrm{E}+00$ & +00 & & & & +00 & $E+00$ & +00 & +00 & +00 & $E+00$ & \\
\hline PO4 & & & & -02 & -02 & & & & & E-02 & E-02 & E-03 & E-03 & $\mathrm{E}-03$ & E-03 \\
\hline SO4 & $9.74 \mathrm{E}-01$ & $9.74 \mathrm{E}-01$ & $9.74 \mathrm{E}-01$ & $5.92 \mathrm{E}-01$ & $5.92 \mathrm{E}-01$ & $9.74 \mathrm{E}-01$ & $9.74 \mathrm{E}-01$ & $5.92 \mathrm{E}-01$ & $5.92 \mathrm{E}-01$ & $5.92 \mathrm{E}-01$ & $5.92 \mathrm{E}-01$ & $9.74 \mathrm{E}-01$ & $9.74 \mathrm{E}-01$ & $9.74 \mathrm{E}-01$ & $9.74 \mathrm{E}-01$ \\
\hline $\mathbf{C a}$ & $51 \mathrm{E}-04$ & $1.51 \mathrm{E}-04$ & & $1.00 \mathrm{E}-04$ & $1.00 \mathrm{E}-04$ & $1 \mathrm{E}-04$ & & & & $0 \mathrm{E}-04$ & $1.00 \mathrm{E}+00$ & $1 \mathrm{E}-04$ & & $51 \mathrm{E}-04$ & $1.51 \mathrm{E}-04$ \\
\hline $\mathrm{Cl}$ & & & & & & & & & & & & & & $E-01$ & \\
\hline $\mathbf{F}$ & & & & & & & & & & & $E-01$ & & & E-01 & 01 \\
\hline $\mathrm{K}$ & $43 \mathrm{E}-02$ & $1.43 \mathrm{E}-02$ & $1.43 \mathrm{E}-02$ & -02 & -02 & $1.43 \mathrm{E}-02$ & -02 & & & -02 & E-02 & $1.43 \mathrm{E}-02$ & 1.43E-02 & $1.43 \mathrm{E}-02$ & $1.43 \mathrm{E}-02$ \\
\hline NH4 & --- & --- & --- & $4.53 \mathrm{E}-03$ & $4.53 \mathrm{E}-03$ & --- & --- & $4.53 \mathrm{E}-03$ & $4.53 \mathrm{E}-03$ & $4.53 \mathrm{E}-03$ & $4.53 \mathrm{E}-03$ & --- & --- & --- & --- \\
\hline
\end{tabular}


Table 2: Supernate Composition

\begin{tabular}{|c|c|c|c|c|c|c|c|c|c|c|c|c|c|c|c|}
\hline \multicolumn{16}{|c|}{ Supernate Composition (M) } \\
\hline & Tank 25 & Tank 27 & Tank 28 & Tank 29 & Tank 31 & Tank 33 & \begin{tabular}{|l|} 
Tank 34 \\
\end{tabular} & Tank 36 & Tank 37 & Tank 38 & Tank 41 & Tank 44 & Tank 45 & Tank 46 & Tank 47 \\
\hline $\mathrm{Na}$ & $1.19 \mathrm{E}+01$ & $1.30 \mathrm{E}+01$ & $1.24 \mathrm{E}+01$ & $4.87 \mathrm{E}+00$ & $1.27 \mathrm{E}+01$ & $3.07 \mathrm{E}+00$ & $1.08 \mathrm{E}+01$ & $1.27 \mathrm{E}+01$ & $1.27 \mathrm{E}+01$ & $1.48 \mathrm{E}+00$ & $9.94 \mathrm{E}+00$ & $1.41 \mathrm{E}+01$ & $1.33 \mathrm{E}+01$ & $1.50 \mathrm{E}+01$ & $8.56 \mathrm{E}+00$ \\
\hline$\overline{\mathrm{Al}(\mathrm{OH}) 4}$ & $5.00 \mathrm{E}-01$ & $1.08 \mathrm{E}+00$ & $5.10 \mathrm{E}-01$ & $3.20 \mathrm{E}-01$ & $8.60 \mathrm{E}-01$ & $1.00 \mathrm{E}-02$ & $2.90 \mathrm{E}-01$ & $1.00 \mathrm{E}-02$ & $4.80 \mathrm{E}-01$ & $1.60 \mathrm{E}-01$ & $2.00 \mathrm{E}-01$ & $3.80 \mathrm{E}-01$ & $2.20 \mathrm{E}-01$ & $7.00 \mathrm{E}-02$ & $4.00 \mathrm{E}-01$ \\
\hline $\mathrm{CO3}$ & $1.30 \mathrm{E}-01$ & $1.20 \mathrm{E}-01$ & $4.00 \mathrm{E}-02$ & $1.00 \mathrm{E}-02$ & $5.00 \mathrm{E}-02$ & $1.00 \mathrm{E}-02$ & $5.30 \mathrm{E}-01$ & $8.00 \mathrm{E}-02$ & $6.00 \mathrm{E}-02$ & $1.00 \mathrm{E}-01$ & $2.60 \mathrm{E}-01$ & $2.00 \mathrm{E}-01$ & $2.90 \mathrm{E}-01$ & $1.00 \mathrm{E}-02$ & $2.80 \mathrm{E}-01$ \\
\hline $\mathrm{C2O4}$ & $6.80 \mathrm{E}-03$ & $6.70 \mathrm{E}-03$ & $6.80 \mathrm{E}-03$ & $6.40 \mathrm{E}-03$ & $6.80 \mathrm{E}-03$ & $5.70 \mathrm{E}-03$ & $7.40 \mathrm{E}-03$ & $6.80 \mathrm{E}-03$ & $1.70 \mathrm{E}-02$ & $5.90 \mathrm{E}-03$ & $5.70 \mathrm{E}-03$ & $6.80 \mathrm{E}-03$ & $6.70 \mathrm{E}-03$ & $5.70 \mathrm{E}-03$ & $6.80 \mathrm{E}-03$ \\
\hline NO2 & $1.30 \mathrm{E}+00$ & $1.42 \mathrm{E}+00$ & $1.72 \mathrm{E}+00$ & $8.79 \mathrm{E}-01$ & $2.50 \mathrm{E}+00$ & $4.70 \mathrm{E}-02$ & $1.16 \mathrm{E}+00$ & $2.24 \mathrm{E}+00$ & $1.96 \mathrm{E}+00$ & $3.42 \mathrm{E}-01$ & $9.89 \mathrm{E}-01$ & $1.52 \mathrm{E}+00$ & $1.70 \mathrm{E}+00$ & $1.58 \mathrm{E}+00$ & $6.20 \mathrm{E}-01$ \\
\hline NO3 & $1.34 \mathrm{E}+00$ & $1.71 \mathrm{E}+00$ & $2.10 \mathrm{E}+00$ & $8.49 \mathrm{E}-01$ & $2.39 \mathrm{E}+00$ & $1.34 \mathrm{E}+00$ & $2.47 \mathrm{E}+00$ & $1.66 \mathrm{E}+00$ & $2.29 \mathrm{E}+00$ & $3.59 \mathrm{E}-01$ & $3.30 \mathrm{E}+00$ & $1.13 \mathrm{E}+00$ & $1.22 \mathrm{E}+00$ & $1.73 \mathrm{E}+00$ & $2.37 \mathrm{E}+00$ \\
\hline $\mathrm{OH}$ & $9.22 \mathrm{E}+00$ & $9.54 \mathrm{E}+00$ & $7.04 \mathrm{E}+00$ & $1.97 \mathrm{E}+00$ & $5.22 \mathrm{E}+00$ & $1.22 \mathrm{E}+00$ & $6.16 \mathrm{E}+00$ & $1.05 \mathrm{E}+01$ & $7.00 \mathrm{E}+00$ & $1.71 \mathrm{E}+00$ & $3.95 \mathrm{E}+00$ & $1.18 \mathrm{E}+01$ & $1.31 \mathrm{E}+01$ & $6.39 \mathrm{E}+00$ & $3.76 \mathrm{E}+00$ \\
\hline $\mathrm{PO4}$ & $1.80 \mathrm{E}-02$ & $1.80 \mathrm{E}-02$ & $2.40 \mathrm{E}-02$ & $7.30 \mathrm{E}-03$ & $2.20 \mathrm{E}-02$ & $5.30 \mathrm{E}-03$ & $6.90 \mathrm{E}-03$ & $3.10 \mathrm{E}-02$ & $1.60 \mathrm{E}-02$ & $5.50 \mathrm{E}-03$ & $2.63 \mathrm{E}-02$ & $1.20 \mathrm{E}-02$ & $1.30 \mathrm{E}-02$ & $1.76 \mathrm{E}-02$ & $6.30 \mathrm{E}-03$ \\
\hline SO4 & $5.00 \mathrm{E}-03$ & $8.60 \mathrm{E}-03$ & $7.60 \mathrm{E}-03$ & $9.70 \mathrm{E}-03$ & $1.60 \mathrm{E}-02$ & $5.15 \mathrm{E}-02$ & $3.00 \mathrm{E}-02$ & $4.20 \mathrm{E}-03$ & $1.50 \mathrm{E}-02$ & $8.60 \mathrm{E}-03$ & $4.86 \mathrm{E}-02$ & $4.20 \mathrm{E}-03$ & $5.00 \mathrm{E}-03$ & $1.87 \mathrm{E}-02$ & $3.90 \mathrm{E}-02$ \\
\hline $\mathbf{A g}$ & $8.34 \mathrm{E}-07$ & $4.91 \mathrm{E}-06$ & $3.62 \mathrm{E}-06$ & $1.95 \mathrm{E}-06$ & $1.69 \mathrm{E}-06$ & $1.69 \mathrm{E}-06$ & $1.69 \mathrm{E}-06$ & $1.69 \mathrm{E}-06$ & $1.69 \mathrm{E}-06$ & $2.13 \mathrm{E}-06$ & $1.69 \mathrm{E}-06$ & $1.69 \mathrm{E}-06$ & $1.69 \mathrm{E}-06$ & $1.69 \mathrm{E}-06$ & $1.69 \mathrm{E}-06$ \\
\hline As & $1.47 \mathrm{E}-04$ & $1.33 \mathrm{E}-04$ & $1.74 \mathrm{E}-04$ & $2.54 \mathrm{E}-04$ & $2.66 \mathrm{E}-03$ & $1.16 \mathrm{E}-03$ & $3.01 \mathrm{E}-03$ & $4.61 \mathrm{E}-03$ & $3.32 \mathrm{E}-03$ & $1.74 \mathrm{E}-04$ & $2.18 \mathrm{E}-03$ & $5.12 \mathrm{E}-03$ & $5.60 \mathrm{E}-03$ & $3.09 \mathrm{E}-03$ & $2.11 \mathrm{E}-03$ \\
\hline $\mathrm{Ba}$ & $1.46 \mathrm{E}-07$ & $1.46 \mathrm{E}-06$ & $1.09 \mathrm{E}-06$ & $1.02 \mathrm{E}-05$ & \begin{tabular}{|l|}
--- \\
\end{tabular} & ---- & \begin{tabular}{|l|}
--- \\
\end{tabular} & \begin{tabular}{|l|}
--- \\
\end{tabular} & \begin{tabular}{|l|}
--- \\
\end{tabular} & $3.64 \mathrm{E}-07$ & --- & \begin{tabular}{|l|}
--- \\
\end{tabular} & -- & - & --- \\
\hline $\mathrm{Ca}$ & $-\overline{-1}$ & $\mid---$ & \begin{tabular}{|l|}
---- \\
\end{tabular} & $5.29 \mathrm{E}-05$ & --- & 6.91E-05 & --- & --- & --- & $5.85 \mathrm{E}-05$ & \begin{tabular}{|c|}
$9.97 \mathrm{E}-06$ \\
\end{tabular} & ---- & --- & --- & $1.41 \mathrm{E}-05$ \\
\hline Cd & $4.00 \mathrm{E}-06$ & $3.83 \mathrm{E}-06$ & $5.07 \mathrm{E}-06$ & $6.67 \mathrm{E}-06$ & \begin{tabular}{|c|}
$7.15 \mathrm{E}-05$ \\
\end{tabular} & $1.39 \mathrm{E}-05$ & \begin{tabular}{|r|}
$81 \mathrm{E}-05$ \\
\end{tabular} & $1.47 \mathrm{E}-04$ & \begin{tabular}{|c|}
$9.72 \mathrm{E}-05$ \\
\end{tabular} & $3.56 \mathrm{E}-06$ & $5.32 \mathrm{E}-05$ & $1.67 \mathrm{E}-04$ & $1.85 \mathrm{E}-04$ & $8.84 \mathrm{E}-05$ & $5 \mathrm{E}-05$ \\
\hline $\mathrm{Cl}$ & $2.00 \mathrm{E}-02$ & $2.10 \mathrm{E}-02$ & $2.10 \mathrm{E}-02$ & $5.30 \mathrm{E}-03$ & $9.80 \mathrm{E}-03$ & $2.80 \mathrm{E}-03$ & $1.60 \mathrm{E}-02$ & $1.90 \mathrm{E}-02$ & $1.30 \mathrm{E}-02$ & $2.90 \mathrm{E}-03$ & $1.16 \mathrm{E}-02$ & $2.40 \mathrm{E}-02$ & $2.80 \mathrm{E}-02$ & $2.24 \mathrm{E}-02$ & $0 \mathrm{E}-03$ \\
\hline Co & $2.13 \mathrm{E}-05$ & $2.18 \mathrm{E}-05$ & $1.79 \mathrm{E}-05$ & $1.01 \mathrm{E}-05$ & $1.51 \mathrm{E}-05$ & $8.94 \mathrm{E}-06$ & $1.66 \mathrm{E}-05$ & $2.32 \mathrm{E}-05$ & $1.79 \mathrm{E}-05$ & $9.70 \mathrm{E}-06$ & $1.32 \mathrm{E}-05$ & $2.53 \mathrm{E}-05$ & $2.73 \mathrm{E}-05$ & $9 \mathrm{E}-05$ & $9 \mathrm{E}-05$ \\
\hline $\mathrm{Cr}$ & 8.85E-04 & $5.39 \mathrm{E}-04$ & $1.79 \mathrm{E}-03$ & $7.83 \mathrm{E}-03$ & $1.06 \mathrm{E}-02$ & $1.18 \mathrm{E}-02$ & $1.03 \mathrm{E}-02$ & $9.02 \mathrm{E}-03$ & $1.01 \mathrm{E}-02$ & $9.79 \mathrm{E}-03$ & $1.10 \mathrm{E}-02$ & $8.61 \mathrm{E}-03$ & $8.22 \mathrm{E}-03$ & $1.03 \mathrm{E}-02$ & $1 \mathrm{E}-02$ \\
\hline $\mathrm{Cu}$ & $7.66 \mathrm{E}-03$ & $7.93 \mathrm{E}-03$ & $5.80 \mathrm{E}-03$ & $1.47 \mathrm{E}-03$ & $4.24 \mathrm{E}-03$ & $8.32 \mathrm{E}-04$ & 55E-03 & $8.72 \mathrm{E}-03$ & EE-03 & $1.25 \mathrm{E}-03$ & 6E-03 & $9.87 \mathrm{E}-03$ & 0E-02 & $5.24 \mathrm{E}-03$ & $0 \mathrm{E}-03$ \\
\hline $\mathrm{Fe}$ & E-05 & 6.4 & E-05 & E-05 & E-04 & $\mathrm{E}-05$ & E-04 & E-04 & E-04 & E-05 & E-04 & $2 \mathrm{E}-04$ & E-04 & $2.37 \mathrm{E}-04$ & $8 \mathrm{E}-04$ \\
\hline $\bar{F}$ & $1.30 \mathrm{E}-02$ & $1.30 \mathrm{E}-02$ & $1.30 \mathrm{E}-02$ & $5.90 \mathrm{E}-03$ & $1.30 \mathrm{E}-02$ & $5.90 \mathrm{E}-03$ & $1.30 \mathrm{E}-02$ & $1.30 \mathrm{E}-02$ & $1.30 \mathrm{E}-02$ & $5.50 \mathrm{E}-03$ & $5.20 \mathrm{E}-03$ & $1.30 \mathrm{E}-02$ & $1.30 \mathrm{E}-02$ & $7.80 \mathrm{E}-03$ & $\overline{\mathrm{EE}-02}$ \\
\hline $\mathrm{Hg}$ & $4.99 \mathrm{E}-05$ & $4.99 \mathrm{E}-05$ & $4.99 \mathrm{E}-05$ & $9.97 \mathrm{E}-05$ & $6.13 \mathrm{E}-04$ & $5.82 \mathrm{E}-04$ & $6.20 \mathrm{E}-04$ & $6.53 \mathrm{E}-04$ & E-04 & $4.99 \mathrm{E}-05$ & 3E-04 & 3E-04 & $3 \mathrm{E}-04$ & $6.22 \mathrm{E}-04$ & $2 \mathrm{E}-04$ \\
\hline $\mathbf{K}$ & $1.30 \mathrm{E}-01$ & $1.40 \mathrm{E}-01$ & $1.30 \mathrm{E}-01$ & $4.10 \mathrm{E}-02$ & $7.60 \mathrm{E}-02$ & $1.30 \mathrm{E}-02$ & $7.60 \mathrm{E}-02$ & $1.70 \mathrm{E}-01$ & $8.70 \mathrm{E}-02$ & $1.33 \mathrm{E}-02$ & $3.70 \mathrm{E}-02$ & $1.60 \mathrm{E}-01$ & $1.80 \mathrm{E}-01$ & $8.60 \mathrm{E}-02$ & $5.40 \mathrm{E}-02$ \\
\hline $\mathrm{Mg}$ & \begin{tabular}{|l|}
---- \\
\end{tabular} & ---- & $4.70 \mathrm{E}-07$ & $6.44 \mathrm{E}-06$ & $2.61 \mathrm{E}-06$ & $7.32 \mathrm{E}-06$ & $1.51 \mathrm{E}-06$ & ---- & $5.17 \mathrm{E}-07$ & $6.74 \mathrm{E}-06$ & $4.11 \mathrm{E}-06$ & \begin{tabular}{|l|}
--- \\
\end{tabular} & ---- & $1.23 \mathrm{E}-06$ & $4.33 \mathrm{E}-06$ \\
\hline Mn & $2.73 \mathrm{E}-06$ & $3.28 \mathrm{E}-06$ & $4.37 \mathrm{E}-06$ & $5.46 \mathrm{E}-06$ & $9.99 \mathrm{E}-06$ & $4.73 \mathrm{E}-06$ & $1.12 \mathrm{E}-05$ & $1.69 \mathrm{E}-05$ & $1.23 \mathrm{E}-05$ & $2.91 \mathrm{E}-06$ & $8.32 \mathrm{E}-06$ & $1.87 \mathrm{E}-05$ & 4E-05 & $1.15 \mathrm{E}-05$ & $8.07 \mathrm{E}-06$ \\
\hline Nd & --- & ---- & ---- & $2.14 \mathrm{E}-04$ & ---- & $2.65 \mathrm{E}-04$ & ---- & ---- & ---- & $2.31 \mathrm{E}-04$ & $7.95 \mathrm{E}-05$ & ---- & ---- & --- & $9.24 \mathrm{E}-05$ \\
\hline $\mathrm{Ni}$ & $5.11 \mathrm{E}-05$ & 5.24E-05 & $4.21 \mathrm{E}-05$ & $2.12 \mathrm{E}-05$ & \begin{tabular}{|c|}
$3.46 \mathrm{E}-05$ \\
\end{tabular} & $1.81 \mathrm{E}-05$ & \begin{tabular}{|c|}
$3.85 \mathrm{E}-05$ \\
\end{tabular} & $5.62 \mathrm{E}-05$ & \begin{tabular}{|c|}
$4.19 \mathrm{E}-05$ \\
\end{tabular} & $2.01 \mathrm{E}-05$ & $2.94 \mathrm{E}-05$ & $6.18 \mathrm{E}-05$ & 6.71E-05 & $3.94 \mathrm{E}-05$ & $2.86 \mathrm{E}-05$ \\
\hline $\mathbf{P b}$ & $1.40 \mathrm{E}-04$ & $1.16 \mathrm{E}-04$ & $1.59 \mathrm{E}-04$ & $2.46 \mathrm{E}-04$ & \begin{tabular}{|l|}
--- \\
\end{tabular} & ---- & \begin{tabular}{|l|}
--- \\
\end{tabular} & $\mid---$ & \begin{tabular}{|l|}
--- \\
\end{tabular} & $1.45 \mathrm{E}-04$ & \begin{tabular}{|l|}
--- \\
\end{tabular} & \begin{tabular}{|l|}
---- \\
\end{tabular} & \begin{tabular}{|l|}
---- \\
\end{tabular} & \begin{tabular}{|l}
---- \\
\end{tabular} & \begin{tabular}{|l|}
--- \\
\end{tabular} \\
\hline $\mathbf{R u}$ & $8.08 \mathrm{E}-04$ & $8.35 \mathrm{E}-04$ & $6.27 \mathrm{E}-04$ & $2.05 \mathrm{E}-04$ & \begin{tabular}{|r|}
$4.75 \mathrm{E}-04$ \\
\end{tabular} & 1.42E-04 & \begin{tabular}{|r|}
$5.53 \mathrm{E}-04$ \\
\end{tabular} & \begin{tabular}{|l|}
$9.12 \mathrm{E}-04$ \\
\end{tabular} & \begin{tabular}{|c|}
$6.23 \mathrm{E}-04$ \\
\end{tabular} & $1.83 \mathrm{E}-04$ & $3.69 \mathrm{E}-04$ & $1.02 \mathrm{E}-03$ & $1.13 \mathrm{E}-03$ & 5.73E-04 & $3.54 \mathrm{E}-04$ \\
\hline Se & $1.04 \mathrm{E}-03$ & $7.98 \mathrm{E}-04$ & $1.34 \mathrm{E}-03$ & $2.98 \mathrm{E}-03$ & $8.15 \mathrm{E}-03$ & $9.87 \mathrm{E}-03$ & $7.75 \mathrm{E}-03$ & $5.91 \mathrm{E}-03$ & $7.39 \mathrm{E}-03$ & $2.85 \mathrm{E}-03$ & $8.70 \mathrm{E}-03$ & $5.33 \mathrm{E}-03$ & $4.78 \mathrm{E}-03$ & $7.65 \mathrm{E}-03$ & $8.78 \mathrm{E}-03$ \\
\hline Si & $4.95 \mathrm{E}-03$ & $4.34 \mathrm{E}-03$ & $2.54 \mathrm{E}-02$ & $8.90 \mathrm{E}-04$ & \begin{tabular}{|l|}
--- \\
\end{tabular} & ---- & \begin{tabular}{|l|}
--- \\
\end{tabular} & $\mid---$ & --- & $6.94 \mathrm{E}-03$ & \begin{tabular}{|l|}
--- \\
\end{tabular} & ---- & --- & ---- & ---- \\
\hline $\mathrm{Sr}$ & \begin{tabular}{|l|}
--- \\
\end{tabular} & $\mid----$ & \begin{tabular}{|l|}
---- \\
\end{tabular} & $1.44 \mathrm{E}-04$ & ---- & $1.83 \mathrm{E}-04$ & --- & --- & --- & $1.57 \mathrm{E}-04$ & $3.98 \mathrm{E}-05$ & ---- & \begin{tabular}{|c|}
--- \\
\end{tabular} & ---- & $4.98 \mathrm{E}-05$ \\
\hline Zn & $9.30 \mathrm{E}-03$ & $9.59 \mathrm{E}-03$ & $7.28 \mathrm{E}-03$ & $2.58 \mathrm{E}-03$ & $5.59 \mathrm{E}-03$ & $1.88 \mathrm{E}-03$ & \begin{tabular}{|r|}
$6.46 \mathrm{E}-03$ \\
\end{tabular} & \begin{tabular}{|r|}
$1.05 \mathrm{E}-02$ \\
\end{tabular} & $7.24 \mathrm{E}-03$ & $2.34 \mathrm{E}-03$ & $4.41 \mathrm{E}-03$ & $1.17 \mathrm{E}-02$ & $1.29 \mathrm{E}-02$ & $6.68 \mathrm{E}-03$ & $4.24 \mathrm{E}-03$ \\
\hline $\mathrm{Zr}$ & $2.45 \mathrm{E}-04$ & $2.53 \mathrm{E}-04$ & $1.87 \mathrm{E}-04$ & $5.21 \mathrm{E}-05$ & $1.38 \mathrm{E}-04$ & $3.22 \mathrm{E}-05$ & $1.63 \mathrm{E}-04$ & $2.78 \mathrm{E}-04$ & $1.86 \mathrm{E}-04$ & $4.52 \mathrm{E}-05$ & $1.05 \mathrm{E}-04$ & $3.14 \mathrm{E}-04$ & $3.48 \mathrm{E}-04$ & $1.70 \mathrm{E}-04$ & $9.96 \mathrm{E}-05$ \\
\hline
\end{tabular}


Table 2: Supernate Composition (continued)

\begin{tabular}{|c|c|c|c|c|c|c|c|c|c|c|c|c|c|c|c|}
\hline \multicolumn{16}{|c|}{ Radionuclide Composition (Ci/gal) } \\
\hline & Tank 25 & Tank 27 & Tank 28 & Tank 29 & Tank 31 & Tank 33 & Tank 34 & Tank 36 & Tank 37 & Tank 38 & Tank 41 & Tank 44 & Tank 45 & Tank 46 & Tank 47 \\
\hline Sr-90 & $2.90 \mathrm{E}-03$ & $2.90 \mathrm{E}-03$ & $2.90 \mathrm{E}-03$ & $1.10 \mathrm{E}-02$ & $1.10 \mathrm{E}-02$ & $1.10 \mathrm{E}-02$ & $1.10 \mathrm{E}-02$ & $1.10 \mathrm{E}-02$ & $1.10 \mathrm{E}-02$ & $2.90 \mathrm{E}-03$ & $2.90 \mathrm{E}-03$ & $2.90 \mathrm{E}-03$ & $2.90 \mathrm{E}-03$ & $2.90 \mathrm{E}-03$ & $2.90 \mathrm{E}-03$ \\
\hline Y-90 & $2.90 \mathrm{E}-03$ & $2.90 \mathrm{E}-03$ & $2.90 \mathrm{E}-03$ & $1.10 \mathrm{E}-02$ & $1.10 \mathrm{E}-02$ & $1.10 \mathrm{E}-02$ & $1.10 \mathrm{E}-02$ & $1.10 \mathrm{E}-02$ & $1.10 \mathrm{E}-02$ & $2.90 \mathrm{E}-03$ & $2.90 \mathrm{E}-03$ & $2.90 \mathrm{E}-03$ & $2.90 \mathrm{E}-03$ & $2.90 \mathrm{E}-03$ & $2.90 \mathrm{E}-03$ \\
\hline Cs-137 & $4.29 \mathrm{E}+00$ & $4.07 \mathrm{E}+00$ & $4.47 \mathrm{E}+00$ & $4.09 \mathrm{E}+00$ & $1.14 \mathrm{E}+01$ & $9.93 \mathrm{E}-02$ & $3.48 \mathrm{E}+00$ & $2.09 \mathrm{E}+01$ & $1.52 \mathrm{E}+01$ & $2.70 \mathrm{E}-01$ & $2.52 \mathrm{E}+00$ & $5.30 \mathrm{E}+00$ & $5.01 \mathrm{E}+00$ & $6.47 \mathrm{E}+00$ & $2.67 \mathrm{E}+00$ \\
\hline Ba-137m & $4.06 \mathrm{E}+00$ & $3.85 \mathrm{E}+00$ & $4.23 \mathrm{E}+00$ & $3.87 \mathrm{E}+00$ & $1.07 \mathrm{E}+01$ & $9.40 \mathrm{E}-02$ & $3.29 \mathrm{E}+00$ & $1.98 \mathrm{E}+01$ & $1.44 \mathrm{E}+01$ & $2.56 \mathrm{E}-01$ & $2.39 \mathrm{E}+00$ & $5.01 \mathrm{E}+00$ & $4.74 \mathrm{E}+00$ & $6.12 \mathrm{E}+00$ & $2.52 \mathrm{E}+00$ \\
\hline Th-232 & $-\cdots$ & 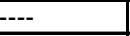 & $---\cdot$ & $5.39 \mathrm{E}-10$ & $5.39 \mathrm{E}-10$ & --- & $-\cdots$ & $5.39 \mathrm{E}-10$ & $5.39 \mathrm{E}-10$ & $---\cdot$ & --- & $-\cdots$ & --- & $-\cdots$ & -- \\
\hline $\mathrm{U}-232$ & $3.48 \mathrm{E}-09$ & $3.48 \mathrm{E}-09$ & $3.48 \mathrm{E}-09$ & $1.53 \mathrm{E}-10$ & $1.53 \mathrm{E}-10$ & $4.59 \mathrm{E}-08$ & $4.66 \mathrm{E}-08$ & $1.53 \mathrm{E}-10$ & $1.53 \mathrm{E}-10$ & --- & $-\overline{-}$ & $3.48 \mathrm{E}-09$ & $3.48 \mathrm{E}-09$ & $3.48 \mathrm{E}-09$ & $3.48 \mathrm{E}-09$ \\
\hline $\mathbf{U}-233$ & --- & --- & ---- & $1.10 \mathrm{E}-07$ & $1.10 \mathrm{E}-07$ & --- & --- & $1.10 \mathrm{E}-07$ & $1.10 \mathrm{E}-07$ & $-\cdots$ & --- & --- & 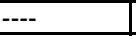 & ---- & 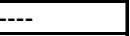 \\
\hline U-234 & --- & ---- & --- & $1.56 \mathrm{E}-08$ & $1.56 \mathrm{E}-08$ & --- & ---- & $1.56 \mathrm{E}-08$ & $1.56 \mathrm{E}-08$ & $2.55 \mathrm{E}-07$ & $2.55 \mathrm{E}-07$ & 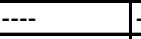 & ---- & ---- & --- \\
\hline U-235 & $\begin{array}{l}7.79 \mathrm{E}-09 \\
\end{array}$ & $\begin{array}{l}7.79 \mathrm{E}-09 \\
\end{array}$ & $\begin{array}{l}7.79 \mathrm{E}-09 \\
\end{array}$ & $4.26 \mathrm{E}-10$ & $4.26 \mathrm{E}-10$ & 5.44E-08 & $\begin{array}{l}4.36 \mathrm{E}-08 \\
\end{array}$ & $4.26 \mathrm{E}-10$ & $4.26 \mathrm{E}-10$ & $2.57 \mathrm{E}-09$ & $2.57 \mathrm{E}-09$ & $7.79 \mathrm{E}-09$ & $7.79 \mathrm{E}-09$ & $7.79 \mathrm{E}-09$ & $7.79 \mathrm{E}-09$ \\
\hline U-236 & ---- & ---- & $\begin{array}{ll}--- \\
-\end{array}$ & $1.61 \mathrm{E}-09$ & $1.61 \mathrm{E}-09$ & --- & --- & $1.61 \mathrm{E}-09$ & $1.61 \mathrm{E}-09$ & $3.88 \mathrm{E}-08$ & $3.88 \mathrm{E}-08$ & --. & ---- & - & --- \\
\hline $\mathrm{U}-238$ & $7.12 \mathrm{E}-07$ & $7.12 \mathrm{E}-07$ & $7.12 \mathrm{E}-07$ & 4.98E-09 & $4.98 \mathrm{E}-09$ & $4.97 \mathrm{E}-06$ & $3.08 \mathrm{E}-06$ & $4.98 \mathrm{E}-09$ & $4.98 \mathrm{E}-09$ & $3.60 \mathrm{E}-10$ & $3.60 \mathrm{E}-10$ & 7.12E-07 & $7.12 \mathrm{E}-07$ & $7.12 \mathrm{E}-07$ & $7.12 \mathrm{E}-07$ \\
\hline Np-237 & -- & -- & -- & $4.01 \mathrm{E}-08$ & $4.01 \mathrm{E}-08$ & $4.10 \mathrm{E}-06$ & $2.38 \mathrm{E}-06$ & $4.01 \mathrm{E}-08$ & $4.01 \mathrm{E}-08$ & $2.22 \mathrm{E}-07$ & $2.22 \mathrm{E}-07$ & -- & -- & -- & --- \\
\hline Pur-238 & $5.90 \mathrm{E}-03$ & $5.90 \mathrm{E}-03$ & $5.90 \mathrm{E}-03$ & 1.53E-04 & 1.53E-04 & --- & --- & $1.53 \mathrm{E}-04$ & $1.53 \mathrm{E}-04$ & $7.00 \mathrm{E}-03$ & $7.00 \mathrm{E}-03$ & $5.90 \mathrm{E}-03$ & $5.90 \mathrm{E}-03$ & $5.90 \mathrm{E}-03$ & $5.90 \mathrm{E}-03$ \\
\hline Pur-239 & $8.43 \mathrm{E}-04$ & $8.43 \mathrm{E}-04$ & $8.43 \mathrm{E}-04$ & $3.96 \mathrm{E}-06$ & $3.96 \mathrm{E}-06$ & $5.95 \mathrm{E}-04$ & $\begin{array}{l}4.98 \mathrm{E}-04 \\
\end{array}$ & $3.96 \mathrm{E}-06$ & $3.96 \mathrm{E}-06$ & $2.16 \mathrm{E}-05$ & $2.16 \mathrm{E}-05$ & $8.43 \mathrm{E}-04$ & $8.43 \mathrm{E}-04$ & $8.43 \mathrm{E}-04$ & $8.43 \mathrm{E}-04$ \\
\hline Pur-240 & $1.88 \mathrm{E}-04$ & $1.88 \mathrm{E}-04$ & $1.88 \mathrm{E}-04$ & $1.64 \mathrm{E}-06$ & $1.64 \mathrm{E}-06$ & 1.33E-04 & $1.11 \mathrm{E}-04$ & $1.64 \mathrm{E}-06$ & $1.64 \mathrm{E}-06$ & $1.43 \mathrm{E}-05$ & $1.43 \mathrm{E}-05$ & $1.88 \mathrm{E}-04$ & $1.88 \mathrm{E}-04$ & $1.88 \mathrm{E}-04$ & $1.88 \mathrm{E}-04$ \\
\hline Pu-241 & $5.05 \mathrm{E}-03$ & $5.05 \mathrm{E}-03$ & $5.05 \mathrm{E}-03$ & $3.56 \mathrm{E}-05$ & $3.56 \mathrm{E}-05$ & 3.46E-03 & $2.83 \mathrm{E}-03$ & 3.56E-05 & 3.56E-05 & 1.19E-03 & $1.19 \mathrm{E}-03$ & 5.05E-03 & 5.05E-03 & $5.05 \mathrm{E}-03$ & $5.05 \mathrm{E}-03$ \\
\hline Pur-242 & $3.87 \mathrm{E}-08$ & $3.87 \mathrm{E}-08$ & $3.87 \mathrm{E}-08$ & $1.09 \mathrm{E}-09$ & $1.09 \mathrm{E}-09$ & $2.74 \mathrm{E}-08$ & $2.31 \mathrm{E}-08$ & $1.09 \mathrm{E}-09$ & $\mathrm{E}-09$ & $2.68 \mathrm{E}-07$ & $2.68 \mathrm{E}-07$ & $3.87 \mathrm{E}-08$ & $3.87 \mathrm{E}-08$ & $3.87 \mathrm{E}-08$ & $3.87 \mathrm{E}-08$ \\
\hline Am-241 & ---- & --- & ---- & $4.50 \mathrm{E}-05$ & $4.50 \mathrm{E}-05$ & $6.24 \mathrm{E}-03$ & $6.38 \mathrm{E}-03$ & $4.50 \mathrm{E}-05$ & $4.50 \mathrm{E}-05$ & $\begin{array}{l}3.74 \mathrm{E}-05 \\
\end{array}$ & $3.74 \mathrm{E}-05$ & ---- & ---- & ---- & ---- \\
\hline Am-242m & $-\cdots$ & --- & --- & $5.50 \mathrm{E}-08$ & $5.50 \mathrm{E}-08$ & $8.52 \mathrm{E}-06$ & $8.68 \mathrm{E}-06$ & $5.50 \mathrm{E}-08$ & $5.50 \mathrm{E}-08$ & $4.65 \mathrm{E}-08$ & $4.65 \mathrm{E}-08$ & \begin{tabular}{ll}
--- \\
\hdashline-
\end{tabular} & $-\cdots-$ & $-\cdots$ & $-\cdots$ \\
\hline $\mathrm{Cm}-244$ & 7.32E-08 & 7.32E-08 & 7.32E-08 & $1.32 \mathrm{E}-07$ & $1.32 \mathrm{E}-07$ & 3.31E-06 & 3.26E-06 & $1.32 \mathrm{E}-07$ & $1.32 \mathrm{E}-07$ & 6.31E-07 & $6.31 \mathrm{E}-07$ & 7.32E-08 & $7.32 \mathrm{E}-08$ & $7.32 \mathrm{E}-08$ & 7.32E-08 \\
\hline $\mathrm{Cm}-245$ & $2.88 \mathrm{E}-14$ & $2.88 \mathrm{E}-14$ & $2.88 \mathrm{E}-14$ & $1.63 \mathrm{E}-11$ & $1.63 \mathrm{E}-11$ & $1.30 \mathrm{E}-12$ & $1.33 \mathrm{E}-12$ & $1.63 \mathrm{E}-11$ & $1.63 \mathrm{E}-11$ & $3.88 \mathrm{E}-11$ & $3.88 \mathrm{E}-11$ & $2.88 \mathrm{E}-14$ & $2.88 \mathrm{E}-14$ & $2.88 \mathrm{E}-14$ & $2.88 \mathrm{E}-14$ \\
\hline Total Alpha & $6.93 \mathrm{E}-03$ & $6.93 \mathrm{E}-03$ & $6.93 \mathrm{E}-03$ & $2.04 \mathrm{E}-04$ & $2.04 \mathrm{E}-04$ & $6.98 \mathrm{E}-03$ & $7.00 \mathrm{E}-03$ & $2.04 \mathrm{E}-04$ & $2.04 \mathrm{E}-04$ & $7.08 \mathrm{E}-03$ & $7.08 \mathrm{E}-03$ & $6.93 \mathrm{E}-03$ & $6.93 \mathrm{E}-03$ & $6.93 \mathrm{E}-03$ & $6.93 \mathrm{E}-03$ \\
\hline
\end{tabular}

Notes:

- $\mathrm{Sr} / \mathrm{Y}$ concentrations are extracted from reference 4 due to limited data within WCS.

- Cs concentrations were converted to $\mathrm{Ci} / \mathrm{gal}$ from the Cs concentrations in $\mathrm{mg} / \mathrm{L}$ given in WCS, using a Specific Activity of $8.70 \mathrm{E}-2 \mathrm{Ci} / \mathrm{mg}$.

- $\mathrm{Ba}-137 \mathrm{~m}$ concentrations assumed to be $94.6 \%$ of the $\mathrm{Cs}-137$ concentration. 
Table 3: Average Radionuclide Concentration in Sludge

\begin{tabular}{|l|r|}
\hline \multicolumn{1}{|c|}{ Nuclides } & $\begin{array}{c}\text { Sludge Solids } \\
\text { (Ci/mg) }\end{array}$ \\
\hline C-14 & $1.49 \mathrm{E}-12$ \\
\hline Ni-59 & $8.55 \mathrm{E}-10$ \\
\hline Ni-63 & $1.33 \mathrm{E}-12$ \\
\hline Co-60 & $1.36 \mathrm{E}-07$ \\
\hline Se-79 & $5.04 \mathrm{E}-10$ \\
\hline Tc-99 & $8.58 \mathrm{E}-09$ \\
\hline Ru-106 & $3.17 \mathrm{E}-09$ \\
\hline Rh-106 & $3.17 \mathrm{E}-09$ \\
\hline Sb-125 & $8.11 \mathrm{E}-08$ \\
\hline Sn-126 & $6.74 \mathrm{E}-10$ \\
\hline $\mathrm{I}-129$ & $7.89 \mathrm{E}-14$ \\
\hline Cs-134 & $3.55 \mathrm{E}-09$ \\
\hline Cs-135 & $6.03 \mathrm{E}-12$ \\
\hline Cs-137 & $1.79 \mathrm{E}-06$ \\
\hline Ba-137m & $1.70 \mathrm{E}-06$ \\
\hline Sr-90 & $2.97 \mathrm{E}-05$ \\
\hline Y-90 & $2.97 \mathrm{E}-05$ \\
\hline Ce-144 & $1.63 \mathrm{E}-09$ \\
\hline Pr-144 & $1.63 \mathrm{E}-09$ \\
\hline Pm-147 & $1.71 \mathrm{E}-06$ \\
\hline Eu-154 & $3.33 \mathrm{E}-07$ \\
\hline Th-232 & $9.57 \mathrm{E}-13$ \\
\hline $\mathrm{U}-232$ & $2.06 \mathrm{E}-13$ \\
\hline $\mathrm{U}-233$ & $3.24 \mathrm{E}-11$ \\
\hline $\mathrm{U}-234$ & $1.04 \mathrm{E}-11$ \\
\hline $\mathrm{U}-235$ & $5.12 \mathrm{E}-13$ \\
\hline $\mathrm{U}-236$ & $1.47 \mathrm{E}-12$ \\
\hline $\mathrm{U}-238$ & $1.57 \mathrm{E}-11$ \\
\hline Np-237 & $2.24 \mathrm{E}-11$ \\
\hline Pu-238 & $6.31 \mathrm{E}-07$ \\
\hline Pu-239 & $1.08 \mathrm{E}-08$ \\
\hline Pu-240 & $5.04 \mathrm{E}-09$ \\
\hline Pu-241 & $3.44 \mathrm{E}-07$ \\
\hline Pu-242 & $9.84 \mathrm{E}-12$ \\
\hline Am-241 & $7.12 \mathrm{E}-08$ \\
\hline Am-242m & $6.69 \mathrm{E}-11$ \\
\hline Cm-244 & \\
\hline Cm-245-12 \\
\hline
\end{tabular}

Notes:

- $\quad$ Sludge radionuclide distribution derived from HLW Waste Characterization Database. 
Table 4: Projected Feed Composition After Dissolution and Dilution to 6.44 M Total Sodium Concentration $600 \mathrm{mg} / \mathrm{L}$ sludge; $30 \%$ of interstitial supernate remaining

\begin{tabular}{|c|c|c|c|c|c|c|c|c|c|c|c|c|c|c|c|}
\hline \multicolumn{16}{|c|}{ Concentration (M) } \\
\hline & Tank 25 & Tank 27 & Tank 28 & Tank 29 & Tank 31 & Tank 33 & \begin{tabular}{|l|} 
Tank 34 \\
\end{tabular} & \begin{tabular}{|l|} 
Tank 36 \\
\end{tabular} & Tank 37 & Tank 38 & Tank 41 & Tank 44 & Tank 45 & Tank 46 & Tank 47 \\
\hline $\mathrm{Na}$ & $6.44 \mathrm{E}+00$ & $6.44 \mathrm{E}+00$ & $6.44 \mathrm{E}+00$ & $6.44 \mathrm{E}+00$ & $6.44 \mathrm{E}+00$ & $6.44 \mathrm{E}+00$ & $6.44 \mathrm{E}+00$ & $6.44 \mathrm{E}+00$ & $6.44 \mathrm{E}+00$ & $6.44 \mathrm{E}+00$ & $6.44 \mathrm{E}+00$ & $6.44 \mathrm{E}+00$ & $6.44 \mathrm{E}+00$ & $6.44 \mathrm{E}+00$ & $6.44 \mathrm{E}+00$ \\
\hline $\mathrm{Al}(\mathrm{OH}) 4$ & $3.20 \mathrm{E}-01$ & $3.32 \mathrm{E}-01$ & $3.20 \mathrm{E}-01$ & $4.00 \mathrm{E}-01$ & $4.01 \mathrm{E}-01$ & $3.19 \mathrm{E}-01$ & $3.17 \mathrm{E}-01$ & 3.84E-01 & $3.93 \mathrm{E}-01$ & $4.01 \mathrm{E}-01$ & $3.91 \mathrm{E}-01$ & $3.15 \mathrm{E}-01$ & $3.13 \mathrm{E}-01$ & $3.08 \mathrm{E}-01$ & $3.22 \mathrm{E}-01$ \\
\hline $\mathrm{CO}$ & $2.18 \mathrm{E}-01$ & $2.17 \mathrm{E}-01$ & $2.16 \mathrm{E}-01$ & $1.32 \mathrm{E}-01$ & $1.30 \mathrm{E}-01$ & $2.22 \mathrm{E}-01$ & $2.27 \mathrm{E}-01$ & $1.30 \mathrm{E}-01$ & $1.30 \mathrm{E}-01$ & $1.35 \mathrm{E}-01$ & $1.35 \mathrm{E}-01$ & $2.18 \mathrm{E}-01$ & $2.20 \mathrm{E}-01$ & $2.13 \mathrm{E}-01$ & $2.24 \mathrm{E}-01$ \\
\hline C2O4 & $2.18 \mathrm{E}-02$ & $2.17 \mathrm{E}-02$ & $2.18 \mathrm{E}-02$ & $1.41 \mathrm{E}-02$ & $1.38 \mathrm{E}-02$ & $2.24 \mathrm{E}-02$ & $2.19 \mathrm{E}-02$ & $1.38 \mathrm{E}-02$ & $1.40 \mathrm{E}-02$ & $1.43 \mathrm{E}-02$ & $1.39 \mathrm{E}-02$ & $2.17 \mathrm{E}-02$ & $2.17 \mathrm{E}-02$ & $2.16 \mathrm{E}-02$ & $2.21 \mathrm{E}-02$ \\
\hline NO2 & $1.26 \mathrm{E}-01$ & $1.28 \mathrm{E}-01$ & $1.35 \mathrm{E}-01$ & $7.48 \mathrm{E}-01$ & $7.62 \mathrm{E}-01$ & $1.02 \mathrm{E}-01$ & $1.24 \mathrm{E}-01$ & $7.57 \mathrm{E}-01$ & $7.52 \mathrm{E}-01$ & $7.45 \mathrm{E}-01$ & $7.39 \mathrm{E}-01$ & $1.30 \mathrm{E}-01$ & $1.34 \mathrm{E}-01$ & $1.31 \mathrm{E}-01$ & $1.13 \mathrm{E}-01$ \\
\hline NO3 & $3.72 \mathrm{E}+00$ & $3.71 \mathrm{E}+00$ & $3.73 \mathrm{E}+00$ & $2.65 \mathrm{E}+00$ & $2.61 \mathrm{E}+00$ & $3.83 \mathrm{E}+00$ & $3.76 \mathrm{E}+00$ & $2.60 \mathrm{E}+00$ & $2.61 \mathrm{E}+00$ & $2.67 \mathrm{E}+00$ & $2.65 \mathrm{E}+00$ & $3.69 \mathrm{E}+00$ & $3.70 \mathrm{E}+00$ & $3.69 \mathrm{E}+00$ & $3.78 \mathrm{E}+00$ \\
\hline $\mathrm{OH}$ & $1.07 \mathrm{E}+00$ & $1.07 \mathrm{E}+00$ & $1.02 \mathrm{E}+00$ & $1.86 \mathrm{E}+00$ & $1.88 \mathrm{E}+00$ & $9.25 \mathrm{E}-01$ & $1.01 \mathrm{E}+00$ & $1.98 \mathrm{E}+00$ & $1.91 \mathrm{E}+00$ & $1.87 \mathrm{E}+00$ & $1.87 \mathrm{E}+00$ & $1.12 \mathrm{E}+00$ & $1.15 \mathrm{E}+00$ & $9.99 \mathrm{E}-01$ & $9.63 \mathrm{E}-01$ \\
\hline $\mathrm{PO4}$ & $1.64 \mathrm{E}-03$ & $1.63 \mathrm{E}-03$ & $1.77 \mathrm{E}-03$ & $1.56 \mathrm{E}-02$ & $1.55 \mathrm{E}-02$ & $1.41 \mathrm{E}-03$ & $1.41 \mathrm{E}-03$ & \begin{tabular}{|c|}
$1.57 \mathrm{E}-02$ \\
\end{tabular} & $1.54 \mathrm{E}-02$ & $1.58 \mathrm{E}-02$ & $1.58 \mathrm{E}-02$ & $1.50 \mathrm{E}-03$ & $1.53 \mathrm{E}-03$ & $1.61 \mathrm{E}-03$ & $1.40 \mathrm{E}-03$ \\
\hline SO4 & $3.17 \mathrm{E}-01$ & $3.16 \mathrm{E}-01$ & $3.16 \mathrm{E}-01$ & $1.82 \mathrm{E}-01$ & $1.78 \mathrm{E}-01$ & $3.27 \mathrm{E}-01$ & $3.18 \mathrm{E}-01$ & $1.78 \mathrm{E}-01$ & $1.78 \mathrm{E}-01$ & $1.84 \mathrm{E}-01$ & $1.80 \mathrm{E}-01$ & $3.14 \mathrm{E}-01$ & $3.15 \mathrm{E}-01$ & $3.14 \mathrm{E}-01$ & $3.21 \mathrm{E}-01$ \\
\hline $\mathrm{Ag}$ & $1.79 \mathrm{E}-08$ & $1.05 \mathrm{E}-07$ & $7.74 \mathrm{E}-08$ & $3.95 \mathrm{E}-08$ & $3.34 \mathrm{E}-08$ & $3.73 \mathrm{E}-08$ & $\mathrm{E}-08$ & $3.34 \mathrm{E}-08$ & $3.34 \mathrm{E}-08$ & $4.38 \mathrm{E}-08$ & $3.37 \mathrm{E}-08$ & $3.59 \mathrm{E}-08$ & $3.60 \mathrm{E}-08$ & $3.58 \mathrm{E}-08$ & $3.66 \mathrm{E}-08$ \\
\hline As & $3.15 \mathrm{E}-06$ & $2.85 \mathrm{E}-06$ & $3.72 \mathrm{E}-06$ & $5.15 \mathrm{E}-06$ & $5.26 \mathrm{E}-05$ & $2.57 \mathrm{E}-05$ & $6.47 \mathrm{E}-05$ & $9.14 \mathrm{E}-05$ & $6.58 \mathrm{E}-05$ & $3.56 \mathrm{E}-06$ & $4.36 \mathrm{E}-05$ & $1.09 \mathrm{E}-04$ & $1.20 \mathrm{E}-04$ & $6.56 \mathrm{E}-05$ & $4.58 \mathrm{E}-05$ \\
\hline $\mathrm{Ba}$ & $3.12 \mathrm{E}-09$ & $3.11 \mathrm{E}-08$ & $2.34 \mathrm{E}-08$ & $2.07 \mathrm{E}-07$ & ---- & \begin{tabular}{|l}
---- \\
\end{tabular} & ---- & \begin{tabular}{|l}
---- \\
\end{tabular} & ---- & $7.47 \mathrm{E}-09$ & --- & ---- & --- & ---- & ---- \\
\hline $\mathrm{Ca}$ & $4.92 \mathrm{E}-05$ & $4.90 \mathrm{E}-05$ & $4.91 \mathrm{E}-05$ & $3.19 \mathrm{E}-05$ & $3.01 \mathrm{E}-05$ & \begin{tabular}{|c|}
$5.22 \mathrm{E}-05$ \\
\end{tabular} & $4.93 \mathrm{E}-05$ & 3.01E-05 & $3.01 \mathrm{E}-05$ & $3.24 \mathrm{E}-05$ & 3.03E-01 & $4.88 \mathrm{E}-05$ & $4.89 \mathrm{E}-05$ & $4.87 \mathrm{E}-05$ & $5.00 \mathrm{E}-05$ \\
\hline $\mathrm{Cd}$ & $8.59 \mathrm{E}-08$ & $8.18 \mathrm{E}-08$ & $1.09 \mathrm{E}-07$ & 1.35E-07 & $1.42 \mathrm{E}-06$ & $3.08 \mathrm{E}-07$ & $1.83 \mathrm{E}-06$ & $2.92 \mathrm{E}-06$ & $1.93 \mathrm{E}-06$ & $7.30 \mathrm{E}-08$ & $1.06 \mathrm{E}-06$ & $3.55 \mathrm{E}-06$ & $3.95 \mathrm{E}-06$ & $1.88 \mathrm{E}-06$ & $1.10 \mathrm{E}-06$ \\
\hline $\mathrm{Cl}$ & $4.19 \mathrm{E}-02$ & $4.18 \mathrm{E}-02$ & $4.18 \mathrm{E}-02$ & 3.83E-02 & 3.74E-02 & $4.28 \mathrm{E}-02$ & $4.20 \mathrm{E}-02$ & $3.76 \mathrm{E}-02$ & 3.75E-02 & 3.86E-02 & $3.78 \mathrm{E}-02$ & 4.17E-02 & $4.19 \mathrm{E}-02$ & 4.15E-02 & $4.21 \mathrm{E}-02$ \\
\hline Co & $4.57 \mathrm{E}-07$ & $4.66 \mathrm{E}-07$ & $3.84 \mathrm{E}-07$ & $2.05 \mathrm{E}-07$ & $3.00 \mathrm{E}-07$ & $1.98 \mathrm{E}-07$ & -07 & $4.60 \mathrm{E}-07$ & $3.54 \mathrm{E}-07$ & $1.99 \mathrm{E}-07$ & $2.63 \mathrm{E}-07$ & $5.39 \mathrm{E}-07$ & $5.84 \mathrm{E}-07$ & $3.60 \mathrm{E}-07$ & $2.79 \mathrm{E}-07$ \\
\hline $\mathrm{Cr}$ & $1.90 \mathrm{E}-05$ & $1.15 \mathrm{E}-05$ & $3.83 \mathrm{E}-05$ & $1.59 \mathrm{E}-04$ & $2.10 \mathrm{E}-04$ & $2.61 \mathrm{E}-04$ & $2.22 \mathrm{E}-04$ & $1.79 \mathrm{E}-04$ & $2.00 \mathrm{E}-04$ & $2.01 \mathrm{E}-04$ & $2.20 \mathrm{E}-04$ & $1.83 \mathrm{E}-04$ & $1.76 \mathrm{E}-04$ & $2.18 \mathrm{E}-04$ & $2.40 \mathrm{E}-04$ \\
\hline $\mathrm{Cu}$ & $1.64 \mathrm{E}-04$ & $1.69 \mathrm{E}-04$ & $1.24 \mathrm{E}-04$ & $2.99 \mathrm{E}-05$ & $8.41 \mathrm{E}-05$ & $1.84 \mathrm{E}-05$ & 1.09E-04 & $1.73 \mathrm{E}-04$ & $1.14 \mathrm{E}-04$ & $2.56 \mathrm{E}-05$ & $6.31 \mathrm{E}-05$ & $2.10 \mathrm{E}-04$ & $2.34 \mathrm{E}-04$ & $1.11 \mathrm{E}-04$ & $6.50 \mathrm{E}-05$ \\
\hline $\mathrm{Fe}$ & $1.38 \mathrm{E}-06$ & $1.38 \mathrm{E}-06$ & 1.07E-06 & $8.36 \mathrm{E}-07$ & $3.83 \mathrm{E}-06$ & $9.41 \mathrm{E}-07$ & $4.92 \mathrm{E}-06$ & $7.74 \mathrm{E}-06$ & $5.15 \mathrm{E}-06$ & $1.95 \mathrm{E}-06$ & $2.90 \mathrm{E}-06$ & $9.40 \mathrm{E}-06$ & $1.05 \mathrm{E}-05$ & $5.04 \mathrm{E}-06$ & $3.00 \mathrm{E}-06$ \\
\hline $\mathbf{F}$ & $6.95 \mathrm{E}-02$ & $6.93 \mathrm{E}-02$ & $6.94 \mathrm{E}-02$ & $3.59 \mathrm{E}-02$ & $3.52 \mathrm{E}-02$ & $7.15 \mathrm{E}-02$ & $6.98 \mathrm{E}-02$ & $3.52 \mathrm{E}-02$ & $3.52 \mathrm{E}-02$ & $3.63 \mathrm{E}-02$ & $3.53 \mathrm{E}-02$ & $6.90 \mathrm{E}-02$ & $6.92 \mathrm{E}-02$ & $6.87 \mathrm{E}-02$ & $7.03 \mathrm{E}-02$ \\
\hline $\mathrm{Hg}$ & 1.07E-06 & 1.07E-06 & 1.07E-06 & $2.02 \mathrm{E}-06$ & $1.21 \mathrm{E}-05$ & $1.29 \mathrm{E}-05$ & $1.34 \mathrm{E}-05$ & $1.29 \mathrm{E}-05$ & $1.24 \mathrm{E}-05$ & $1.02 \mathrm{E}-06$ & $1.20 \mathrm{E}-05$ & $1.41 \mathrm{E}-05$ & $1.44 \mathrm{E}-05$ & $1.32 \mathrm{E}-05$ & $1.31 \mathrm{E}-05$ \\
\hline $\mathrm{K}$ & $7.45 \mathrm{E}-03$ & $7.64 \mathrm{E}-03$ & $7.44 \mathrm{E}-03$ & $5.24 \mathrm{E}-03$ & $5.81 \mathrm{E}-03$ & $5.09 \mathrm{E}-03$ & $6.32 \mathrm{E}-03$ & $7.67 \mathrm{E}-03$ & $6.03 \mathrm{E}-03$ & $4.73 \mathrm{E}-03$ & $5.08 \mathrm{E}-03$ & $8.04 \mathrm{E}-03$ & $8.48 \mathrm{E}-03$ & $6.44 \mathrm{E}-03$ & $5.89 \mathrm{E}-03$ \\
\hline $\mathrm{Mg}$ & ---- & ---- & $1.01 \mathrm{E}-08$ & $1.31 \mathrm{E}-07$ & 5.17E-08 & 1.62E-07 & $3.24 \mathrm{E}-08$ & --- & $1.02 \mathrm{E}-08$ & $1.38 \mathrm{E}-07$ & $8.20 \mathrm{E}-08$ & --- & --- & $2.62 \mathrm{E}-08$ & 9.39E-08 \\
\hline $\mathrm{Mn}$ & $5.86 \mathrm{E}-08$ & 7.00E-08 & $9.36 \mathrm{E}-08$ & $1.11 \mathrm{E}-07$ & $1.98 \mathrm{E}-07$ & $1.04 \mathrm{E}-07$ & $2.42 \mathrm{E}-07$ & 3.35E-07 & $2.44 \mathrm{E}-07$ & $5.98 \mathrm{E}-08$ & $1.66 \mathrm{E}-07$ & $3.98 \mathrm{E}-07$ & $4.35 \mathrm{E}-07$ & $2.45 \mathrm{E}-07$ & $1.75 \mathrm{E}-07$ \\
\hline Nd & ---- & \begin{tabular}{|l|}
---- \\
\end{tabular} & $-\cdots$ & $4.34 \mathrm{E}-06$ & ---- & $5.85 \mathrm{E}-06$ & --- & \begin{tabular}{|l|}
--- \\
\end{tabular} & $\begin{array}{ll}--- \\
\end{array}$ & $4.75 \mathrm{E}-06$ & $1.59 \mathrm{E}-06$ & \begin{tabular}{|l|}
--- \\
\end{tabular} & ---- & $\begin{array}{ll}---- \\
\end{array}$ & $2.00 \mathrm{E}-06$ \\
\hline $\mathrm{Ni}$ & $1.10 \mathrm{E}-06$ & $1.12 \mathrm{E}-06$ & $9.01 \mathrm{E}-07$ & $4.30 \mathrm{E}-07$ & $6.85 \mathrm{E}-07$ & $4.00 \mathrm{E}-07$ & $8.28 \mathrm{E}-07$ & $1.11 \mathrm{E}-06$ & $8.30 \mathrm{E}-07$ & $4.13 \mathrm{E}-07$ & 5.87E-07 & $1.32 \mathrm{E}-06$ & $1.43 \mathrm{E}-06$ & $8.37 \mathrm{E}-07$ & $6.20 \mathrm{E}-07$ \\
\hline $\mathrm{Pb}$ & $3.00 \mathrm{E}-06$ & $2.48 \mathrm{E}-06$ & $3.41 \mathrm{E}-06$ & $5.00 \mathrm{E}-06$ & --- & ---- & --- & --- & ---- & $2.97 \mathrm{E}-06$ & --- & $\begin{array}{ll}--- \\
-\end{array}$ & $-\overline{---}$ & \begin{tabular}{|l|}
--- \\
\end{tabular} & --- \\
\hline $\mathbf{R u}$ & $1.73 \mathrm{E}-05$ & $1.78 \mathrm{E}-05$ & $1.34 \mathrm{E}-05$ & 4.15E-06 & $9.41 \mathrm{E}-06$ & $3.14 \mathrm{E}-06$ & $1.19 \mathrm{E}-05$ & 1.81E-05 & $1.23 \mathrm{E}-05$ & $3.76 \mathrm{E}-06$ & $7.38 \mathrm{E}-06$ & $2.18 \mathrm{E}-05$ & $2.42 \mathrm{E}-05$ & $1.22 \mathrm{E}-05$ & 7.67E-06 \\
\hline $\mathrm{Se}$ & $2.23 \mathrm{E}-05$ & $1.71 \mathrm{E}-05$ & $2.87 \mathrm{E}-05$ & $6.04 \mathrm{E}-05$ & $1.62 \mathrm{E}-04$ & $2.18 \mathrm{E}-04$ & $1.67 \mathrm{E}-04$ & $1.17 \mathrm{E}-04$ & $1.46 \mathrm{E}-04$ & $5.85 \mathrm{E}-05$ & $1.74 \mathrm{E}-04$ & $1.13 \mathrm{E}-04$ & $1.02 \mathrm{E}-04$ & $1.62 \mathrm{E}-04$ & $1.90 \mathrm{E}-04$ \\
\hline $\mathrm{Si}$ & 1.06E-04 & $9.29 \mathrm{E}-05$ & 5.44E-04 & $1.81 \mathrm{E}-05$ & -- & ---- & --- & $\begin{array}{ll}--- \\
\end{array}$ & \begin{tabular}{|l|}
---- \\
\end{tabular} & $1.42 \mathrm{E}-04$ & --- & ---- & ---- & \begin{tabular}{|l|}
---- \\
\end{tabular} & -- \\
\hline $\mathrm{Sr}$ & $\begin{array}{ll}--- \\
--\end{array}$ & \begin{tabular}{|l|}
--- \\
\end{tabular} & \begin{tabular}{|l|}
---- \\
\end{tabular} & $2.92 \mathrm{E}-06$ & --- & $4.05 \mathrm{E}-06$ & $-\cdots$ & --- & --- & $3.23 \mathrm{E}-06$ & 7.95E-07 & - & --- & --- & $1.08 \mathrm{E}-06$ \\
\hline $\mathrm{Zn}$ & 1.99E-04 & \begin{tabular}{|l}
$2.05 \mathrm{E}-04$ \\
\end{tabular} & \begin{tabular}{|l|}
$1.56 \mathrm{E}-04$ \\
\end{tabular} & $5.24 \mathrm{E}-05$ & 1.11E-04 & $4.16 \mathrm{E}-05$ & $1.39 \mathrm{E}-04$ & \begin{tabular}{|l|}
$2.07 \mathrm{E}-04$ \\
\end{tabular} & \begin{tabular}{|l}
$1.43 \mathrm{E}-04$ \\
\end{tabular} & $4.80 \mathrm{E}-05$ & $8.82 \mathrm{E}-05$ & $2.49 \mathrm{E}-04$ & $2.76 \mathrm{E}-04$ & 1.42E-04 & $9.19 \mathrm{E}-05$ \\
\hline $\mathrm{Zr}$ & $5.25 \mathrm{E}-06$ & $5.41 \mathrm{E}-06$ & $4.00 \mathrm{E}-06$ & $1.06 \mathrm{E}-06$ & $2.74 \mathrm{E}-06$ & $7.12 \mathrm{E}-07$ & $3.52 \mathrm{E}-06$ & $5.50 \mathrm{E}-06$ & $3.68 \mathrm{E}-06$ & $9.28 \mathrm{E}-07$ & $2.09 \mathrm{E}-06$ & $6.68 \mathrm{E}-06$ & $7.43 \mathrm{E}-06$ & $3.60 \mathrm{E}-06$ & $2.16 \mathrm{E}-06$ \\
\hline Insol.Sol.mg/L & 600 & 600 & 600 & 600 & 600 & 600 & 600 & 600 & 600 & 600 & 600 & 600 & 600 & 600 & 600 \\
\hline
\end{tabular}


Table 4: Projected Feed Composition After Dissolution and Dilution to 6.44 M Total Sodium Concentration $600 \mathrm{mg} / \mathrm{L}$ sludge; $30 \%$ of interstitial supernate remaining (continued)

\begin{tabular}{|c|c|c|c|c|c|c|c|c|c|c|c|c|c|c|c|}
\hline \multicolumn{16}{|c|}{ Radionuclide Composition (Ci/gal) } \\
\hline & Tank 25 & Tank 27 & Tank 28 & Tank 29 & Tank 31 & Tank 33 & Tank 34 & Tank 36 & Tank 37 & Tank 38 & Tank 41 & Tank 44 & Tank 45 & Tank 46 & Tank 47 \\
\hline C-14 & $3.37 \mathrm{E}-09$ & $3.37 \mathrm{E}-09$ & $3.37 \mathrm{E}-09$ & $3.37 \mathrm{E}-09$ & $3.37 \mathrm{E}-09$ & & & $3.37 \mathrm{E}-09$ & $3.37 \mathrm{E}-09$ & $3.37 \mathrm{E}-09$ & $3.37 \mathrm{E}-09$ & $3.37 \mathrm{E}-09$ & $3.37 \mathrm{E}-09$ & $3.37 \mathrm{E}-09$ & $3.37 \mathrm{E}-09$ \\
\hline Ni-59 & & & & $1.94 \mathrm{E}-06$ & $1.94 \mathrm{E}-06$ & $1.94 \mathrm{E}-06$ & $1.94 \mathrm{E}-06$ & $1.94 \mathrm{E}-06$ & $1.94 \mathrm{E}-06$ & $1.94 \mathrm{E}-06$ & $1.94 \mathrm{E}-06$ & $1.94 \mathrm{E}-06$ & $1.94 \mathrm{E}-06$ & $1.94 \mathrm{E}-06$ & $1.94 \mathrm{E}-0$ \\
\hline $\mathrm{Ni}-63$ & $3.01 \mathrm{E}-09$ & $3.01 \mathrm{E}-09$ & $3.01 \mathrm{E}-09$ & $3.01 \mathrm{E}-09$ & $3.01 \mathrm{E}-09$ & $3.01 \mathrm{E}-09$ & $3.01 \mathrm{E}-09$ & $3.01 \mathrm{E}-09$ & $3.01 \mathrm{E}-09$ & $3.01 \mathrm{E}-09$ & $3.01 \mathrm{E}-09$ & $3.01 \mathrm{E}-09$ & $3.01 \mathrm{E}-09$ & $3.01 \mathrm{E}-09$ & $3.01 \mathrm{E}-0$ \\
\hline Co-60 & $3.09 \mathrm{E}-04$ & $3.09 \mathrm{E}-04$ & $3.09 \mathrm{E}-04$ & $3.09 \mathrm{E}-04$ & & & & $3.09 \mathrm{E}-04$ & $3.09 \mathrm{E}-04$ & $3.09 \mathrm{E}-04$ & $3.09 \mathrm{E}-04$ & & $3.09 \mathrm{E}-04$ & & 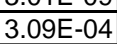 \\
\hline Se-79 & & & & $1.14 \mathrm{E}-06$ & $1.14 \mathrm{E}-06$ & & & $1.14 \mathrm{E}-06$ & $1.14 \mathrm{E}-06$ & $1.14 \mathrm{E}-06$ & $1.14 \mathrm{E}-06$ & & & $1.14 \mathrm{E}-06$ & \\
\hline Tc-99 & $1.94 \mathrm{E}-05$ & $1.94 \mathrm{E}-05$ & $1.94 \mathrm{E}-05$ & $1.94 \mathrm{E}-05$ & $1.94 \mathrm{E}-05$ & $1.94 \mathrm{E}-05$ & $1.94 \mathrm{E}-05$ & $1.94 \mathrm{E}-05$ & $1.94 \mathrm{E}-05$ & $1.94 \mathrm{E}-05$ & $1.94 \mathrm{E}-05$ & $1.94 \mathrm{E}-05$ & $1.94 \mathrm{E}-05$ & $1.94 \mathrm{E}-05$ & $1.94 \mathrm{E}-05$ \\
\hline Ru-106 & $7.18 \mathrm{E}-06$ & $7.18 \mathrm{E}-06$ & $7.18 \mathrm{E}-06$ & $7.18 \mathrm{E}-06$ & $7.18 \mathrm{E}-06$ & & & & & & & & & & \\
\hline Rh-106 & & & & 7.18 & $3 \mathrm{E}-06$ & & & & & $7.18 \mathrm{E}-06$ & & & & & \\
\hline Sb-125 & $1.84 \mathrm{E}-04$ & $1.84 \mathrm{E}-04$ & $1.84 \mathrm{E}-04$ & $1.84 \mathrm{E}-04$ & $1.84 \mathrm{E}-04$ & $1.84 \mathrm{E}-04$ & $1.84 \mathrm{E}-04$ & $1.84 \mathrm{E}-04$ & $1.84 \mathrm{E}-04$ & $1.84 \mathrm{E}-04$ & $1.84 \mathrm{E}-04$ & $1.84 \mathrm{E}-04$ & $1.84 \mathrm{E}-04$ & $1.84 \mathrm{E}-04$ & $1.84 \mathrm{E}-02$ \\
\hline Sn-126 & $1.53 \mathrm{E}-06$ & $1.53 \mathrm{E}-06$ & $1.53 \mathrm{E}-06$ & $1.53 \mathrm{E}-06$ & $1.53 \mathrm{E}-06$ & $1.53 \mathrm{E}-06$ & $1.53 \mathrm{E}-06$ & $1.53 \mathrm{E}-06$ & $1.53 \mathrm{E}-06$ & $1.53 \mathrm{E}-06$ & $1.53 \mathrm{E}-06$ & $1.53 \mathrm{E}-06$ & $1.53 \mathrm{E}-06$ & $1.53 \mathrm{E}-06$ & $1.53 \mathrm{E}-0$ \\
\hline I-129 & & & & & & & & & & & & & & & \\
\hline Cs-134 & E-06 & $8.05 \mathrm{E}-06$ & $8.05 \mathrm{E}-06$ & $8.05 \mathrm{E}-06$ & $8.05 \mathrm{E}-06$ & & $8.05 \mathrm{E}-06$ & $8.05 \mathrm{E}-06$ & $8.05 \mathrm{E}-06$ & $8.05 \mathrm{E}-06$ & $8.05 \mathrm{E}-06$ & $8.05 \mathrm{E}-06$ & $8.05 \mathrm{E}-06$ & $8.05 \mathrm{E}-06$ & \\
\hline Cs-135 & $1.37 \mathrm{E}-08$ & $1.37 \mathrm{E}-08$ & $1.37 \mathrm{E}-08$ & $1.37 \mathrm{E}-08$ & $1.37 \mathrm{E}-08$ & $1.37 \mathrm{E}-08$ & $1.37 \mathrm{E}-08$ & $1.37 \mathrm{E}-08$ & $1.37 \mathrm{E}-08$ & $1.37 \mathrm{E}-08$ & $\frac{0.37 \mathrm{E}-08}{1.08}$ & $\frac{1.03 L-08}{1.37 E-08}$ & $\frac{0.07 L-08}{1.37 E}$ & $\frac{1.37 \mathrm{E}-08}{2}$ & $1.37 \mathrm{E}-08$ \\
\hline Cs-137 & & & & & & & & & & & & & & & \\
\hline Ba-137m & -02 & $8.62 \mathrm{E}-02$ & $9.44 \mathrm{E}-02$ & $8.24 \mathrm{E}-02$ & $\frac{2.25 \mathrm{E}-01}{2.17 \mathrm{E}}$ & 5.93 & 7.47 & $\frac{1.10 \mathrm{~L}-0}{3.96 \mathrm{E}-0}$ & $\frac{0.07 \mathrm{E}-01}{2.88 \mathrm{E}}$ & $9.10 \mathrm{E}-03$ & $5.16 \mathrm{E}-02$ & E-C & $1.05 \mathrm{E}-01$ & $1.34 \mathrm{E}-01$ & \\
\hline Sr-90 & E-02 & $6.74 \mathrm{E}-02$ & $6.74 \mathrm{E}-02$ & $6.76 \mathrm{E}-02$ & $6.75 \mathrm{E}-02$ & 6.76 & & $6.75 \mathrm{E}-02$ & $6.75 \mathrm{E}-02$ & $6.74 \mathrm{E}-02$ & $6.74 \mathrm{E}-02$ & & $6.74 \mathrm{E}-02$ & $4 \mathrm{E}-02$ & $6.74 \mathrm{E}-0$ \\
\hline$Y-90$ & 6.74 & $6.74 \mathrm{E}-02$ & $6.74 \mathrm{E}-02$ & 6.76 & $6.75 \mathrm{E}-02$ & & & $6.75 \mathrm{E}-02$ & $6.75 \mathrm{E}-02$ & 6.74 & & & $=-02$ & & $6.74 \mathrm{E}$ \\
\hline $\mathrm{H}-3$ & & $\cdots$ & -- & -- & --- & -- & --- & --- & -- & -- & --- & --- & $\ldots$ & & $-\cdots$ \\
\hline Ce-144 & $3.70 \mathrm{E}-06$ & $3.70 \mathrm{E}-06$ & $3.70 \mathrm{E}-06$ & $3.70 \mathrm{E}-06$ & $3.70 \mathrm{E}-06$ & $3.70 \mathrm{E}-06$ & $3.70 \mathrm{E}-06$ & $3.70 \mathrm{E}-06$ & $3.70 \mathrm{E}-06$ & $3.70 \mathrm{E}-06$ & $3.70 \mathrm{E}-06$ & $3.70 \mathrm{E}-06$ & $3.70 \mathrm{E}-06$ & $3.70 \mathrm{E}-06$ & \\
\hline Pr-144 & & $3.70 \mathrm{E}-06$ & $3.70 \mathrm{E}-06$ & $3.70 \mathrm{E}-06$ & $3.70 \mathrm{E}-06$ & & & $3.70 \mathrm{E}-06$ & $3.70 \mathrm{E}-06$ & $3.70 \mathrm{E}-06$ & $3.70 \mathrm{E}-06$ & & $3.70 \mathrm{E}-06$ & $3.70 \mathrm{E}-06$ & $3.70 \mathrm{E}-1$ \\
\hline & & & & & & & & & & & & & & & \\
\hline Eu-154 & & $\frac{0.00}{7.56}$ & $\frac{7.06 \mathrm{~L}-04}{7.56 \mathrm{E}-04}$ & $7.56 \mathrm{E}$ & $\frac{0.00 \mathrm{~L}}{7.56 \mathrm{E}-04}$ & & & $7.56 \mathrm{E}-04$ & $7.56 \mathrm{E}-04$ & E-04 & & $\mathrm{E}-04$ & $5 \mathrm{E}-04$ & $7.56 \mathrm{E}-04$ & \\
\hline Th-232 & & & $2.17 \mathrm{E}-09$ & 2.18 & 2.18 & & & $2.18 \mathrm{E}-09$ & & & & & & & 2. \\
\hline-232 & & & 5.43 & & & & & & 4.71 & & & & & $E-10$ & \\
\hline $\mathrm{U}-233$ & & & 7.35 & 7.57 & & & & $7.57 \mathrm{E}-08$ & 7.57 & $E-08$ & & & & $7.35 \mathrm{E}-08$ & $7.35 \mathrm{E}$ \\
\hline U-234 & & & 2.35 & & & & & & & & & & & & \\
\hline U-235 & 1.33 & 1.33 & $1.33 \mathrm{E}-09$ & $1.17 \mathrm{E}-09$ & $1.17 \mathrm{E}-00$ & 2.36 & & $1.17 \mathrm{E}-09$ & 1.17E-09 & E-09 & 1.21 & -09 & 1.33E-09 & $1.33 \mathrm{E}-09$ & $1.33 \mathrm{E}-\mathrm{C}$ \\
\hline $\mathrm{U}-236$ & & & & & & & & & & & & & & & \\
\hline U-238 & & & & & & & & $3.57 \mathrm{E}-0$ & & & & & & & \\
\hline Np-237 & & & 5.08 & & & & & $5.16 \mathrm{E}-08$ & & & & & & & \\
\hline $1-238$ & & & & & & & & & & & & & & & \\
\hline Pu-239 & & & & & & & & 2.47 & & & & & & & \\
\hline Pu-240 & & 1.55 & 1.55 & & & & & $1.15 \mathrm{E}-05$ & & & & & & E-05 & \\
\hline Pu-241 & - -04 & $8.88 \mathrm{E}-04$ & $8.88 \mathrm{E}-04$ & 7.81 & 7.81 & 8.57 & & $7.81 \mathrm{E}-04$ & 7.81 & & & 8.8 & $8.88 \mathrm{E}-04$ & $8.87 \mathrm{E}-04$ & \\
\hline-2 & & & & & & & & & & & & & & & \\
\hline $\mathrm{Am}-24$ & & & $1.62 \mathrm{E}-04$ & & & & & $1.62 \mathrm{E}-04$ & & & & & $1.62 \mathrm{E}-04$ & & \\
\hline Am-24 & $1.52 \mathrm{E}-07$ & $1.52 \mathrm{E}-07$ & $1.52 \mathrm{E}-07$ & $1.53 \mathrm{E}-07$ & $1.53 \mathrm{E}-07$ & $3.40 \mathrm{E}-07$ & $3.39 \mathrm{E}-07$ & $1.53 \mathrm{E}-07$ & $1.53 \mathrm{E}-07$ & $1.53 \mathrm{E}-07$ & $1.53 \mathrm{E}-07$ & $1.52 \mathrm{E}-07$ & $1.52 \mathrm{E}-07$ & $1.52 \mathrm{E}-07$ & $1.52 \mathrm{E}-0$ \\
\hline & & & & & & & & & & & & & & & \\
\hline $1-24$ & & & & & & & & & & & & & & & \\
\hline & $1.24 \mathrm{E}-08$ & $1.24 \mathrm{E}-\mathrm{C}$ & $1.24 \mathrm{E}-08$ & & & & & & & & & & & & \\
\hline & $1.94 \mathrm{E}-03$ & $1.94 \mathrm{E}-03$ & 1.94E-03 & $1.80 \mathrm{E}-03$ & $1.80 \mathrm{E}-03$ & $1.95 \mathrm{E}-03$ & $1.95 \mathrm{E}-03$ & $1.80 \mathrm{E}-03$ & $1.80 \mathrm{E}-03$ & $1.94 \mathrm{E}-03$ & $1.94 \mathrm{E}-03$ & $1.94 \mathrm{E}-03$ & $1.94 \mathrm{E}-03$ & $1.94 \mathrm{E}-03$ & $1.94 \mathrm{E}-0$ \\
\hline
\end{tabular}


Table 5: Projected Feed Composition After Dissolution and Dilution to 6.44 M Total Sodium Concentration $350 \mathrm{mg} / \mathrm{L}$ sludge; $30 \%$ of interstitial supernate remaining

\begin{tabular}{|c|c|c|c|c|c|c|c|c|c|c|c|c|c|c|c|}
\hline \multicolumn{16}{|c|}{ Concentration (M) } \\
\hline & Tank 25 & Tank 27 & Tank 28 & Tank 29 & Tank 31 & Tank 33 & Tank 34 & Tank 36 & Tank 37 & Tank 38 & Tank 41 & Tank 44 & Tank 45 & Tank 46 & Tank 47 \\
\hline $\mathrm{Na}$ & $6.44 \mathrm{E}+00$ & $6.44 \mathrm{E}+00$ & $6.44 \mathrm{E}+00$ & $6.44 \mathrm{E}+00$ & $6.44 \mathrm{E}+00$ & $6.44 \mathrm{E}+00$ & $6.44 \mathrm{E}+00$ & $6.44 \mathrm{E}+00$ & $6.44 \mathrm{E}+00$ & $6.44 \mathrm{E}+00$ & $6.44 \mathrm{E}+00$ & $6.44 \mathrm{E}+00$ & $6.44 \mathrm{E}+00$ & $6.44 \mathrm{E}+00$ & $6.44 \mathrm{E}+00$ \\
\hline $\mathrm{Al}(\mathrm{OH}) 4$ & $3.20 \mathrm{E}-01$ & $3.32 \mathrm{E}-01$ & $3.20 \mathrm{E}-01$ & $4.00 \mathrm{E}-01$ & $4.01 \mathrm{E}-01$ & $3.19 \mathrm{E}-01$ & $3.17 \mathrm{E}-01$ & $3.84 \mathrm{E}-01$ & $3.93 \mathrm{E}-01$ & $4.01 \mathrm{E}-01$ & $3.91 \mathrm{E}-01$ & $3.15 \mathrm{E}-01$ & $3.13 \mathrm{E}-01$ & $3.08 \mathrm{E}-01$ & $3.22 \mathrm{E}-01$ \\
\hline $\mathrm{CO}$ & $2.18 \mathrm{E}-01$ & $2.17 \mathrm{E}-01$ & $2.16 \mathrm{E}-01$ & $1.32 \mathrm{E}-01$ & $1.30 \mathrm{E}-01$ & $2.22 \mathrm{E}-01$ & $2.27 \mathrm{E}-01$ & $1.30 \mathrm{E}-01$ & $1.30 \mathrm{E}-01$ & $1.35 \mathrm{E}-01$ & $1.35 \mathrm{E}-01$ & $2.18 \mathrm{E}-01$ & $2.20 \mathrm{E}-01$ & $2.13 \mathrm{E}-01$ & $2.24 \mathrm{E}-01$ \\
\hline $\mathbf{C 2 O 4}$ & $2.18 \mathrm{E}-02$ & $2.17 \mathrm{E}-02$ & $2.18 \mathrm{E}-02$ & $1.41 \mathrm{E}-02$ & $1.38 \mathrm{E}-02$ & $2.24 \mathrm{E}-02$ & $2.19 \mathrm{E}-02$ & $1.38 \mathrm{E}-02$ & $1.40 \mathrm{E}-02$ & $1.43 \mathrm{E}-02$ & $1.39 \mathrm{E}-02$ & $2.17 \mathrm{E}-02$ & $2.17 \mathrm{E}-02$ & $2.16 \mathrm{E}-02$ & $2.21 \mathrm{E}-02$ \\
\hline NO2 & $1.26 \mathrm{E}-01$ & $1.28 \mathrm{E}-01$ & $1.35 \mathrm{E}-01$ & $7.48 \mathrm{E}-01$ & $7.62 \mathrm{E}-01$ & $1.02 \mathrm{E}-01$ & & $7.57 \mathrm{E}-01$ & $7.52 \mathrm{E}-01$ & $7.45 \mathrm{E}-01$ & $7.39 \mathrm{E}-01$ & $1.30 \mathrm{E}-01$ & $1.34 \mathrm{E}-01$ & $1.31 \mathrm{E}-01$ & $1.13 \mathrm{E}-01$ \\
\hline NO3 & $3.72 \mathrm{E}+00$ & $3.71 \mathrm{E}+00$ & $3.73 \mathrm{E}+00$ & $2.65 \mathrm{E}+00$ & $2.61 \mathrm{E}+00$ & $3.83 \mathrm{E}+00$ & $3.76 \mathrm{E}+00$ & $2.60 \mathrm{E}+00$ & $2.61 \mathrm{E}+00$ & $2.67 \mathrm{E}+00$ & $2.65 \mathrm{E}+00$ & $3.69 \mathrm{E}+00$ & $3.70 \mathrm{E}+00$ & $3.69 \mathrm{E}+00$ & $3.78 \mathrm{E}+00$ \\
\hline $\mathrm{OH}$ & $1.07 \mathrm{E}+00$ & $1.07 \mathrm{E}+00$ & $1.02 \mathrm{E}+00$ & $1.86 \mathrm{E}+00$ & $1.88 \mathrm{E}+00$ & $9.25 \mathrm{E}-01$ & $1.01 \mathrm{E}+00$ & $1.98 \mathrm{E}+00$ & $1.91 \mathrm{E}+00$ & $1.87 \mathrm{E}+00$ & $1.87 \mathrm{E}+00$ & $1.12 \mathrm{E}+00$ & $1.15 \mathrm{E}+00$ & $9.99 \mathrm{E}-01$ & $9.63 \mathrm{E}-01$ \\
\hline$\overline{\mathrm{PO} 4}$ & $1.64 \mathrm{E}-03$ & $1.63 \mathrm{E}-03$ & $1.77 \mathrm{E}-03$ & $1.56 \mathrm{E}-02$ & $1.55 \mathrm{E}-02$ & $1.41 \mathrm{E}-03$ & $1.41 \mathrm{E}-03$ & $1.57 \mathrm{E}-02$ & $1.54 \mathrm{E}-02$ & $1.58 \mathrm{E}-02$ & $1.58 \mathrm{E}-02$ & $1.50 \mathrm{E}-03$ & $1.53 \mathrm{E}-03$ & $1.61 \mathrm{E}-03$ & $1.40 \mathrm{E}-03$ \\
\hline SO4 & $3.17 \mathrm{E}-01$ & $3.16 \mathrm{E}-01$ & $3.16 \mathrm{E}-01$ & $1.82 \mathrm{E}-01$ & $1.78 \mathrm{E}-01$ & $3.27 \mathrm{E}-01$ & & $1.78 \mathrm{E}-01$ & $1.78 \mathrm{E}-01$ & $1.84 \mathrm{E}-01$ & $1.80 \mathrm{E}-01$ & $3.14 \mathrm{E}-01$ & $3.15 \mathrm{E}-01$ & $3.14 \mathrm{E}-01$ & $3.21 \mathrm{E}-01$ \\
\hline$\overline{\mathrm{Ag}}$ & $E-08$ & -07 & $7.74 \mathrm{E}-08$ & -08 & $3.34 \mathrm{E}-08$ & $3.73 \mathrm{E}-08$ & & -08 & $3.34 \mathrm{E}-08$ & E-08 & $3.37 \mathrm{E}-08$ & $9 \mathrm{E}-08$ & $3.60 \mathrm{E}-08$ & $3.58 \mathrm{E}-08$ & $3.66 \mathrm{E}-08$ \\
\hline As & $3.15 \mathrm{E}-06$ & $2.85 \mathrm{E}-06$ & $3.72 \mathrm{E}-06$ & E-06 & $5.26 \mathrm{E}-05$ & $2.57 \mathrm{E}-05$ & $=-05$ & E-05 & $6.58 \mathrm{E}-05$ & $6 \mathrm{E}-06$ & $4.36 \mathrm{E}-05$ & $1.09 \mathrm{E}-04$ & $1.20 \mathrm{E}-04$ & $6.56 \mathrm{E}-05$ & $4.58 \mathrm{E}-05$ \\
\hline $\mathrm{Ba}$ & $3.12 \mathrm{E}-09$ & $3.11 \mathrm{E}-08$ & $2.34 \mathrm{E}-08$ & $2.07 \mathrm{E}-07$ & - & - & $\cdots$ & $-\cdots$ & \begin{tabular}{l|}
--- \\
\end{tabular} & $7.47 \mathrm{E}-09$ & 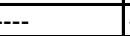 & --- & $-\cdots$ & \begin{tabular}{|l|}
--- \\
\end{tabular} & 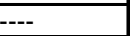 \\
\hline $\mathrm{Ca}$ & $4.92 \mathrm{E}-05$ & $4.90 \mathrm{E}-05$ & $4.91 \mathrm{E}-05$ & $3.19 \mathrm{E}-05$ & $3.01 \mathrm{E}-05$ & $5.22 \mathrm{E}-05$ & $4.93 \mathrm{E}-05$ & $3.01 \mathrm{E}-05$ & $3.01 \mathrm{E}-05$ & $3.24 \mathrm{E}-05$ & $3.03 \mathrm{E}-01$ & $4.88 \mathrm{E}-05$ & $4.89 \mathrm{E}-05$ & \begin{tabular}{|c|}
$4.87 \mathrm{E}-05$ \\
\end{tabular} & $5.00 \mathrm{E}-05$ \\
\hline$\overline{C d}$ & $8.59 \mathrm{E}-08$ & E-08 & $1.09 \mathrm{E}-07$ & E-07 & $1.42 \mathrm{E}-06$ & $3.08 \mathrm{E}-07$ & & E-06 & $1.93 \mathrm{E}-06$ & E-08 & $1.06 \mathrm{E}-06$ & $3.55 \mathrm{E}-06$ & $3.95 \mathrm{E}-06$ & $1.88 \mathrm{E}-06$ & $1.10 \mathrm{E}-06$ \\
\hline $\mathrm{Cl}$ & $4.19 \mathrm{E}-02$ & E-02 & $E-02$ & $=-02$ & $\mathrm{E}-02$ & $E-02$ & & -02 & -02 & E-02 & $3.78 \mathrm{E}-02$ & E-02 & $\mathrm{E}-02$ & $\mathrm{E}-02$ & $4.21 \mathrm{E}-02$ \\
\hline Co & $4.57 \mathrm{E}-07$ & $4.66 \mathrm{E}-07$ & $3.84 \mathrm{E}-07$ & $2.05 \mathrm{E}-07$ & $3.00 \mathrm{E}-07$ & $1.98 \mathrm{E}-07$ & $=-07$ & E-07 & $3.54 \mathrm{E}-07$ & $1.99 \mathrm{E}-07$ & $2.63 \mathrm{E}-07$ & $5.39 \mathrm{E}-07$ & $5.84 \mathrm{E}-07$ & $3.60 \mathrm{E}-07$ & $2.79 \mathrm{E}-07$ \\
\hline $\mathrm{Cr}$ & $1.90 \mathrm{E}-05$ & $1.15 \mathrm{E}-05$ & $3.83 \mathrm{E}-05$ & $1.59 \mathrm{E}-04$ & $2.10 \mathrm{E}-04$ & $2.61 \mathrm{E}-04$ & & E-04 & $2.00 \mathrm{E}-04$ & $2.01 \mathrm{E}-04$ & $2.20 \mathrm{E}-04$ & $1.83 \mathrm{E}-04$ & $1.76 \mathrm{E}-04$ & $2.18 \mathrm{E}-04$ & $2.40 \mathrm{E}-04$ \\
\hline$\overline{\mathrm{Cu}}$ & E-04 & -04 & $1.24 \mathrm{E}-04$ & E-05 & $8.41 \mathrm{E}-05$ & E-05 & -04 & -04 & E-04 & E-05 & $6.31 \mathrm{E}-05$ & $\mathrm{E}-04$ & $2.34 \mathrm{E}-04$ & E-04 & $6.50 \mathrm{E}-05$ \\
\hline $\mathrm{Fe}$ & $1.38 \mathrm{E}-06$ & E-06 & $1.07 \mathrm{E}-06$ & $E-07$ & $3.83 \mathrm{E}-06$ & E-07 & -06 & $E-06$ & $5.15 \mathrm{E}-06$ & $1.95 \mathrm{E}-06$ & $2.90 \mathrm{E}-06$ & $\mathrm{E}-06$ & $1.05 \mathrm{E}-05$ & $\mathrm{E}-06$ & $3.00 \mathrm{E}-06$ \\
\hline $\bar{F}$ & $6.95 \mathrm{E}-02$ & $6.93 \mathrm{E}-02$ & $6.94 \mathrm{E}-02$ & $3.59 \mathrm{E}-02$ & $3.52 \mathrm{E}-02$ & $7.15 \mathrm{E}-02$ & E-02 & $3.52 \mathrm{E}-02$ & 3.52E-02 & $3.63 \mathrm{E}-02$ & $3.53 \mathrm{E}-02$ & $6.90 \mathrm{E}-02$ & $6.92 \mathrm{E}-02$ & $6.87 \mathrm{E}-02$ & $7.03 \mathrm{E}-02$ \\
\hline $\mathrm{Hg}$ & $1.07 \mathrm{E}-06$ & $1.07 \mathrm{E}-06$ & $1.07 \mathrm{E}-06$ & $2.02 \mathrm{E}-06$ & $1.21 \mathrm{E}-05$ & $1.29 \mathrm{E}-05$ & $1.34 \mathrm{E}-05$ & $1.29 \mathrm{E}-05$ & $1.24 \mathrm{E}-05$ & $1.02 \mathrm{E}-06$ & $1.20 \mathrm{E}-05$ & $1.41 \mathrm{E}-05$ & $1.44 \mathrm{E}-05$ & $1.32 \mathrm{E}-05$ & $1.31 \mathrm{E}-05$ \\
\hline $\mathbf{K}$ & $7.45 \mathrm{E}-03$ & $7.64 \mathrm{E}-03$ & $7.44 \mathrm{E}-03$ & $5.24 \mathrm{E}-03$ & $5.81 \mathrm{E}-03$ & $5.09 \mathrm{E}-03$ & $6.32 \mathrm{E}-03$ & $7.67 \mathrm{E}-03$ & $6.03 \mathrm{E}-03$ & $4.73 \mathrm{E}-03$ & $5.08 \mathrm{E}-03$ & $8.04 \mathrm{E}-03$ & $8.48 \mathrm{E}-03$ & $6.44 \mathrm{E}-03$ & $5.89 \mathrm{E}-03$ \\
\hline $\mathbf{M g}$ & --- & --- & -08 & -07 & $5.17 \mathrm{E}-08$ & E-07 & & & $E-08$ & E-07 & $\mathrm{E}-08$ & --- & --- & $2.62 \mathrm{E}-08$ & $9.39 \mathrm{E}-08$ \\
\hline $\mathrm{Mn}$ & $5.86 \mathrm{E}-08$ & $7.00 \mathrm{E}-08$ & $9.36 \mathrm{E}-08$ & E-07 & $\begin{array}{l}1.98 \mathrm{E}-07 \\
\end{array}$ & E-07 & $E-07$ & $3.35 \mathrm{E}-07$ & $2.44 \mathrm{E}-07$ & $5.98 \mathrm{E}-08$ & $1.66 \mathrm{E}-07$ & $3.98 \mathrm{E}-07$ & $4.35 \mathrm{E}-07$ & $2.45 \mathrm{E}-07$ & $1.75 \mathrm{E}-07$ \\
\hline Nd & --- & --- & --- & $4.34 \mathrm{E}-06$ & --- & $5.85 \mathrm{E}-06$ & --- & $-\cdots$ & $-\cdots$ & $4.75 \mathrm{E}-06$ & $1.59 \mathrm{E}-06$ & $\begin{array}{ll}--- \\
\end{array}$ & $\begin{array}{ll}--- \\
\end{array}$ & --- & $2.00 \mathrm{E}-06$ \\
\hline $\mathrm{Ni}$ & $1.10 \mathrm{E}-06$ & $1.12 \mathrm{E}-06$ & $9.01 \mathrm{E}-07$ & $4.30 \mathrm{E}-07$ & $6.85 \mathrm{E}-07$ & $4.00 \mathrm{E}-07$ & $8.28 \mathrm{E}-07$ & $1.11 \mathrm{E}-06$ & $8.30 \mathrm{E}-07$ & $4.13 \mathrm{E}-07$ & $5.87 \mathrm{E}-07$ & $1.32 \mathrm{E}-06$ & $1.43 \mathrm{E}-06$ & 8.37E-07 & $6.20 \mathrm{E}-07$ \\
\hline $\mathbf{P b}$ & $3.00 \mathrm{E}-06$ & $2.48 \mathrm{E}-06$ & $3.41 \mathrm{E}-06$ & $5.00 \mathrm{E}-06$ & --- & -..- & --- & & 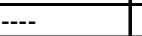 & $2.97 \mathrm{E}-06$ & $\cdots$ & -- & --- & 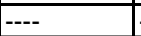 & $-{ }_{-1}$ \\
\hline $\mathbf{R u}$ & $1.73 \mathrm{E}-05$ & $1.78 \mathrm{E}-05$ & $1.34 \mathrm{E}-05$ & $4.15 \mathrm{E}-06$ & $9.41 \mathrm{E}-06$ & $3.14 \mathrm{E}-06$ & -05 & E-05 & $1.23 \mathrm{E}-05$ & $\mathrm{E}-06$ & $7.38 \mathrm{E}-06$ & E-05 & $2.42 \mathrm{E}-05$ & $1.22 \mathrm{E}-05$ & 7.67E-06 \\
\hline $\mathrm{Se}$ & $2.23 \mathrm{E}-05$ & $1.71 \mathrm{E}-05$ & $2.87 \mathrm{E}-05$ & $6.04 \mathrm{E}-05$ & $1.62 \mathrm{E}-04$ & $2.18 \mathrm{E}-04$ & $1.67 \mathrm{E}-04$ & $1.17 \mathrm{E}-04$ & $1.46 \mathrm{E}-04$ & $5.85 \mathrm{E}-05$ & $1.74 \mathrm{E}-04$ & $1.13 \mathrm{E}-04$ & $1.02 \mathrm{E}-04$ & $1.62 \mathrm{E}-04$ & $1.90 \mathrm{E}-04$ \\
\hline Si & $1.06 \mathrm{E}-04$ & $9.29 \mathrm{E}-05$ & $5.44 \mathrm{E}-04$ & $1.81 \mathrm{E}-05$ & 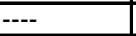 & - & $-{ }_{-1}$ & $-\overline{-}$ & \begin{tabular}{l|l}
--- \\
-
\end{tabular} & $1.42 \mathrm{E}-04$ & \begin{tabular}{l|}
-- \\
-
\end{tabular} & --- & -- & --- & -- \\
\hline $\mathrm{Sr}$ & --- & --- & --- & $2.92 \mathrm{E}-06$ & $-\overline{---}$ & $4.05 \mathrm{E}-06$ & -- & --- & --- & $3.23 \mathrm{E}-06$ & $7.95 \mathrm{E}-07$ & --- & $-\cdots$ & -- & $1.08 \mathrm{E}-06$ \\
\hline$\overline{Z n}$ & $1.99 \mathrm{E}-04$ & $2.05 \mathrm{E}-04$ & $1.56 \mathrm{E}-04$ & $5.24 \mathrm{E}-05$ & $1.11 \mathrm{E}-04$ & $4.16 \mathrm{E}-05$ & $1.39 \mathrm{E}-04$ & $2.07 \mathrm{E}-04$ & $1.43 \mathrm{E}-04$ & $4.80 \mathrm{E}-05$ & $8.82 \mathrm{E}-05$ & $2.49 \mathrm{E}-04$ & $2.76 \mathrm{E}-04$ & $1.42 \mathrm{E}-04$ & $9.19 \mathrm{E}-05$ \\
\hline $\mathrm{Zr}$ & $5.25 \mathrm{E}-06$ & $5.41 \mathrm{E}-06$ & $4.00 \mathrm{E}-06$ & $1.06 \mathrm{E}-06$ & $2.74 \mathrm{E}-06$ & $7.12 \mathrm{E}-07$ & $3.52 \mathrm{E}-06$ & $5.50 \mathrm{E}-06$ & $3.68 \mathrm{E}-06$ & $9.28 \mathrm{E}-07$ & $2.09 \mathrm{E}-06$ & $6.68 \mathrm{E}-06$ & $7.43 \mathrm{E}-06$ & $3.60 \mathrm{E}-06$ & $2.16 \mathrm{E}-06$ \\
\hline Insol.Sol.mg/L & 350 & 350 & 350 & 350 & 350 & 350 & 350 & 350 & 350 & 350 & 350 & 350 & 350 & 350 & 350 \\
\hline
\end{tabular}


Table 5: Projected Feed Composition After Dissolution and Dilution to 6.44 M Total Sodium Concentration $350 \mathrm{mg} / \mathrm{L}$ sludge; $30 \%$ of interstitial supernate remaining (continued)

\begin{tabular}{|c|c|c|c|c|c|c|c|c|c|c|c|c|c|c|c|}
\hline \\
\hline & Tank 25 & Tank 27 & Tank 28 & Tank 29 & Tank 31 & Tank 33 & Tank 34 & Tank 36 & Tank 37 & Tank 38 & Tank 41 & Tank 44 & Tank 45 & Tank 46 & Tank 47 \\
\hline C-14 & $1.97 \mathrm{E}-09$ & $1.97 \mathrm{E}-09$ & $1.97 \mathrm{E}-09$ & $1.97 \mathrm{E}-09$ & $1.97 \mathrm{E}-09$ & $1.97 \mathrm{E}-09$ & 1.97E-09 & 1.97E-09 & 1.97E-09 & $1.97 \mathrm{E}-09$ & $1.97 \mathrm{E}-09$ & $1.97 \mathrm{E}-09$ & $1.97 \mathrm{E}-09$ & 1.97E-09 & $1.97 \mathrm{E}-09$ \\
\hline Ni-59 & $1.13 \mathrm{E}-06$ & $1.13 \mathrm{E}-06$ & $1.13 \mathrm{E}-06$ & $1.13 \mathrm{E}-06$ & $1.13 \mathrm{E}-06$ & $1.13 \mathrm{E}-06$ & $1.13 \mathrm{E}-06$ & 1.13E-06 & $1.13 \mathrm{E}-06$ & $1.13 \mathrm{E}-06$ & $1.13 \mathrm{E}-06$ & $1.13 \mathrm{E}-06$ & $1.13 \mathrm{E}-06$ & $1.13 \mathrm{E}-06$ & $1.13 \mathrm{E}$ \\
\hline $\mathrm{Ni}-63$ & $1.76 \mathrm{E}-09$ & $1.76 \mathrm{E}-09$ & $1.76 \mathrm{E}-09$ & $1.76 \mathrm{E}-09$ & $1.76 \mathrm{E}-09$ & $1.76 \mathrm{E}-09$ & $1.76 \mathrm{E}-09$ & $1.76 \mathrm{E}-09$ & $1.76 \mathrm{E}-09$ & $1.76 \mathrm{E}-09$ & $1.76 \mathrm{E}-09$ & $1.76 \mathrm{E}-09$ & $1.76 \mathrm{E}-09$ & $1.76 \mathrm{E}-09$ & $1.76 \mathrm{E}-0$ \\
\hline Co-60 & $80 \mathrm{E}-04$ & $1.80 \mathrm{E}-04$ & $1.80 \mathrm{E}-04$ & $1.80 \mathrm{E}-04$ & $1.80 \mathrm{E}-04$ & $1.80 \mathrm{E}-04$ & $1.80 \mathrm{E}-04$ & $80 \mathrm{E}-04$ & $1.80 \mathrm{E}-04$ & $1.80 \mathrm{E}-04$ & $1.80 \mathrm{E}-04$ & $1.80 \mathrm{E}-04$ & $.80 \mathrm{E}-04$ & $1.80 \mathrm{E}-04$ & $1.80 \mathrm{E}-0$ \\
\hline Se-79 & 67E-07 & $6.67 \mathrm{E}-07$ & $6.67 \mathrm{E}-07$ & $6.67 \mathrm{E}-07$ & $6.67 \mathrm{E}-07$ & $6.67 \mathrm{E}-07$ & $6.67 \mathrm{E}-07$ & & $6.67 \mathrm{E}-07$ & & $6.67 \mathrm{E}-07$ & & & $6.67 \mathrm{E}-07$ & \\
\hline TC-99 & $1.13 \mathrm{E}-05$ & $1.13 \mathrm{E}-05$ & $1.13 \mathrm{E}-05$ & $1.13 \mathrm{E}-05$ & $1.13 \mathrm{E}-05$ & $1.13 \mathrm{E}-05$ & $1.13 \mathrm{E}-05$ & $1.13 \mathrm{E}-05$ & $1.13 \mathrm{E}-05$ & $1.13 \mathrm{E}-05$ & $1.13 \mathrm{E}-05$ & $1.13 \mathrm{E}-05$ & $1.13 \mathrm{E}-05$ & $1.13 \mathrm{E}-05$ & \\
\hline Ru-106 & $4.19 \mathrm{E}-06$ & $4.19 \mathrm{E}-06$ & $4.19 \mathrm{E}-06$ & $4.19 \mathrm{E}-06$ & $4.19 \mathrm{E}-06$ & $4.19 \mathrm{E}-06$ & $4.19 \mathrm{E}-06$ & $4.19 \mathrm{E}-06$ & $4.19 \mathrm{E}-06$ & $4.19 \mathrm{E}-06$ & $4.19 \mathrm{E}-06$ & $4.19 \mathrm{E}-06$ & $4.19 \mathrm{E}-06$ & $4.19 \mathrm{E}-06$ & $4.19 \mathrm{E}-0 \mathrm{f}$ \\
\hline Rh-106 & & $4.19 \mathrm{E}-06$ & $4.19 \mathrm{E}-06$ & $4.19 \mathrm{E}-06$ & $4.19 \mathrm{E}-06$ & & & & & & & & & & \\
\hline Sb-125 & $07 \mathrm{E}-04$ & $1.07 \mathrm{E}-04$ & $1.07 \mathrm{E}-04$ & $1.07 \mathrm{E}-04$ & $1.07 \mathrm{E}-04$ & $1.07 \mathrm{E}-04$ & $1.07 \mathrm{E}-04$ & $07 \mathrm{E}-04$ & $1.07 \mathrm{E}-04$ & $\frac{1.07 \mathrm{E}-04}{4}$ & $1.07 \mathrm{E}-04$ & $1.07 \mathrm{E}-04$ & $1.07 \mathrm{E}-04$ & $1.07 \mathrm{E}-04$ & \\
\hline Sn-126 & $8.92 \mathrm{E}-07$ & $8.92 \mathrm{E}-07$ & $8.92 \mathrm{E}-07$ & $8.92 \mathrm{E}-07$ & $8.92 \mathrm{E}-07$ & $8.92 \mathrm{E}-07$ & $8.92 \mathrm{E}-07$ & $8.92 \mathrm{E}-07$ & $8.92 \mathrm{E}-07$ & $8.92 \mathrm{E}-07$ & $8.92 \mathrm{E}-07$ & $8.92 \mathrm{E}-07$ & $8.92 \mathrm{E}-07$ & $8.92 \mathrm{E}-07$ & $8.92 \mathrm{E}-$ \\
\hline I-129 & $1.04 \mathrm{E}-10$ & $1.04 \mathrm{E}-10$ & $1.04 \mathrm{E}-10$ & $1.04 \mathrm{E}-10$ & $1.04 \mathrm{E}-10$ & $1.04 \mathrm{E}-10$ & $1.04 \mathrm{E}-10$ & $1.04 \mathrm{E}-10$ & $1.04 \mathrm{E}-10$ & $1.04 \mathrm{E}-10$ & $1.04 \mathrm{E}-10$ & $1.04 \mathrm{E}-10$ & $1.04 \mathrm{E}-10$ & $1.04 \mathrm{E}-10$ & $1.04 \mathrm{E}-1$ \\
\hline Cs-134 & $4.70 \mathrm{E}-06$ & & $4.70 \mathrm{E}-06$ & $4.70 \mathrm{E}-06$ & $4.70 \mathrm{E}-06$ & & & & & & $4.70 \mathrm{E}-06$ & & & $4.70 \mathrm{E}-06$ & \\
\hline Cs-135 & & & $7.98 \mathrm{E}-09$ & $7.98 \mathrm{E}-09$ & $7.98 \mathrm{E}-09$ & & & & $7.98 \mathrm{E}-09$ & $7.98 \mathrm{E}-09$ & $7.98 \mathrm{E}-09$ & 7.98 & 7.9 & $8 \mathrm{E}-09$ & \\
\hline Cs-137 & $9.44 \mathrm{E}-02$ & $8.95 \mathrm{E}-02$ & $9.81 \mathrm{E}-02$ & $8.55 \mathrm{E}-02$ & $2.27 \mathrm{E}-01$ & & & $\mathrm{E}-01$ & $3.03 \mathrm{E}-01$ & $7.92 \mathrm{E}-03$ & $5.28 \mathrm{E}-02$ & $1.15 \mathrm{E}-01$ & $1.09 \mathrm{E}-01$ & $1.40 \mathrm{E}-01$ & \\
\hline Ba-137m & $8.93 \mathrm{E}-02$ & $8.46 \mathrm{E}-02$ & $9.28 \mathrm{E}-02$ & $8.08 \mathrm{E}-02$ & $2.15 \mathrm{E}-01$ & $4.32 \mathrm{E}-03$ & $1 \mathrm{E}-02$ & $4 \mathrm{E}-01$ & 2.87E-01 & $7.50 \mathrm{E}-03$ & $5.00 \mathrm{E}-02$ & $1.09 \mathrm{E}-01$ & $1.04 \mathrm{E}-01$ & $1.32 \mathrm{E}-01$ & $5.70 \mathrm{E}-\mathrm{C}$ \\
\hline & & & $3.93 \mathrm{E}-02$ & & & & & & & & & & & & \\
\hline $1-90$ & $3.93 \mathrm{E}-02$ & $3.93 \mathrm{E}-02$ & $3.93 \mathrm{E}-02$ & $3.95 \mathrm{E}-02$ & $3.95 \mathrm{E}-02$ & $3.95 \mathrm{E}-02$ & $3.95 \mathrm{E}-02$ & $3.95 \mathrm{E}-02$ & $3.95 \mathrm{E}-0$ & $3.93 \mathrm{E}-0$ & $3.93 \mathrm{E}-0$ & $3.93 \mathrm{E}-02$ & $3.93 \mathrm{E}-0$ & $3.93 \mathrm{E}-02$ & $3.93 \mathrm{E}$ \\
\hline $\mathrm{H}-3$ & & - & --- & -- & & --- & $\ldots$ & & --- & --- & & & & & \\
\hline Ce-144 & $2.16 \mathrm{E}-06$ & $2.16 \mathrm{E}-06$ & $2.16 \mathrm{E}-06$ & $2.16 \mathrm{E}-06$ & $2.16 \mathrm{E}-06$ & $2.16 \mathrm{E}-06$ & $2.16 \mathrm{E}-06$ & $2.16 \mathrm{E}-06$ & $2.16 \mathrm{E}-06$ & $2.16 \mathrm{E}-06$ & $2.16 \mathrm{E}-06$ & $2.16 \mathrm{E}-06$ & $2.16 \mathrm{E}-06$ & $2.16 \mathrm{E}-06$ & $2.16 \mathrm{E}$ \\
\hline Pr-144 & $2.16 \mathrm{E}-06$ & 2.16 & $2.16 \mathrm{E}-06$ & $2.16 \mathrm{E}-06$ & $2.16 \mathrm{E}-06$ & & & & $\frac{1.16 \mathrm{E}-06}{2.10}$ & & & & & $2.16 \mathrm{E}-06$ & \\
\hline 0 & & & & & & & & & & & & & & & \\
\hline Eu-154 & $\frac{2.21}{4.41}$ & $=-04$ & $4.41 \mathrm{E}-04$ & $4.41 \mathrm{E}-04$ & $4.41 \mathrm{E}-04$ & & & & & & & & & $1 \mathrm{E}-04$ & \\
\hline Th-232 & & & $1.27 \mathrm{E}-09$ & 1.28 & 1.28 & & & & & & & & & & \\
\hline 232 & & & $3.48 \mathrm{E}-10$ & 2.76 & 2.76 & & & & & & & & & E-10 & \\
\hline & & & & & & & & & & & & & & & \\
\hline U-234 & & & $\begin{array}{l}1.37 \mathrm{E}-08 \\
\end{array}$ & & 1.40 & & & & & & & & & & \\
\hline 235 & & & & & & & & & & & & & & & \\
\hline 36 & & & & & & & & & & & & & & & \\
\hline 238 & 3.61 & & $3.60 \mathrm{E}-$ & $2.09 \mathrm{E}-08$ & $2.09 \mathrm{E}-08$ & & & & $2.09 \mathrm{E}-0$ & & 2.08 & E-08 & & $3.59 \mathrm{E}-08$ & 3.62 \\
\hline & & & & & & & & & & & & & & $2.96 \mathrm{E}-08$ & \\
\hline -238 & & & & & & & & & & & & & & & \\
\hline $1-239$ & 3.24 & & $3.24 \mathrm{E}-05$ & & & & & & & & & 3E-05 & & 3.2 & \\
\hline$u-240$ & 1.07 & $\begin{array}{l}0.5 \\
1.07\end{array}$ & $1.07 \mathrm{E}-05$ & $6.70 \mathrm{E}-06$ & 6.70 & $9.61 \mathrm{E}-06$ & $9.06 \mathrm{E}-06$ & 6.70 & 6.70 & $6.96 \mathrm{E}-06$ & $6.95 \mathrm{E}-06$ & $\begin{array}{l}1.07 \mathrm{E}-05 \\
\end{array}$ & $\frac{0.0}{1.0}$ & & \\
\hline & & & & & & & & & & & & & & & \\
\hline Pu-242 & & & & & & & & & & & & & & $8 \mathrm{E}-08$ & \\
\hline Am-241 & $9.42 \mathrm{E}-05$ & $9.42 \mathrm{E}-05$ & 9.42E-05 & $9.51 \mathrm{E}-05$ & 9.51E-05 & $2.32 \mathrm{E}-04$ & $2.32 \mathrm{E}-04$ & $9.51 \mathrm{E}-05$ & $9.51 \mathrm{E}-05$ & $9.50 \mathrm{E}-05$ & 9.50E-05 & 9.42E-05 & $9.42 \mathrm{E}-05$ & $9.42 \mathrm{E}-05$ & $9.42 \mathrm{E}-$ \\
\hline 24 & $8.85 \mathrm{E}-08$ & $8.85 \mathrm{E}-08$ & $8.85 \mathrm{E}-08$ & $8.97 \mathrm{E}-08$ & $8.96 \mathrm{E}-08$ & $2.77 \mathrm{E}-07$ & $\begin{array}{l}2.75 \mathrm{E}-07 \\
\end{array}$ & $8.96 \mathrm{E}-08$ & $8.96 \mathrm{E}-08$ & $8.95 \mathrm{E}-08$ & $8.95 \mathrm{E}-08$ & $8.85 \mathrm{E}-08$ & $8.85 \mathrm{E}-08$ & $8.85 \mathrm{E}-08$ & $8.85 \mathrm{E}-0$ \\
\hline & & & & & & & & & & & & & & & \\
\hline & & & & & & & & & & & & & & & \\
\hline Cm-245 & $7.21 \mathrm{E}-09$ & $7.21 \mathrm{E}-09$ & $7.21 \mathrm{E}-09$ & $7.21 \mathrm{E}-09$ & $7.21 \mathrm{E}-09$ & & & 7.21 & $7.21 \mathrm{E}-09$ & $7.21 \mathrm{E}-09$ & $7.21 \mathrm{E}-09$ & $7.21 \mathrm{E}-09$ & 7.21 & $7.21 \mathrm{E}-09$ & $7.21 \mathrm{E}$ \\
\hline Total Alpha & $1.20 \mathrm{E}-03$ & $1.20 \mathrm{E}-03$ & $1.20 \mathrm{E}-03$ & $1.05 \mathrm{E}-03$ & $1.05 \mathrm{E}-03$ & $1.20 \mathrm{E}-03$ & $1.20 \mathrm{E}-03$ & $1.05 \mathrm{E}-03$ & $1.05 \mathrm{E}-03$ & $1.19 \mathrm{E}-03$ & $1.19 \mathrm{E}-03$ & $1.19 \mathrm{E}-03$ & $1.19 \mathrm{E}-03$ & $1.19 \mathrm{E}-03$ & $1.20 \mathrm{E}-0$ \\
\hline
\end{tabular}


Table 6: Projected Feed Composition After Dissolution and Dilution to 6.44 M Total Sodium Concentration $100 \mathrm{mg} / \mathrm{L}$ sludge; $30 \%$ of interstitial supernate remaining

\begin{tabular}{|c|c|c|c|c|c|c|c|c|c|c|c|c|c|c|c|}
\hline \multicolumn{16}{|c|}{ Concentration (M) } \\
\hline & Tank 25 & Tank 27 & Tank 28 & Tank 29 & Tank 31 & Tank 33 & Tank 34 & Tank 36 & Tank 37 & Tank 38 & Tank 41 & Tank 44 & Tank 45 & Tank 46 & Tank 47 \\
\hline $\mathrm{Na}$ & $6.44 \mathrm{E}+00$ & $6.44 \mathrm{E}+00$ & $6.44 \mathrm{E}+00$ & $6.44 \mathrm{E}+00$ & $6.44 \mathrm{E}+00$ & $6.44 \mathrm{E}+00$ & $6.44 \mathrm{E}+00$ & $6.44 \mathrm{E}+00$ & $6.44 \mathrm{E}+00$ & $6.44 \mathrm{E}+00$ & $6.44 \mathrm{E}+00$ & $6.44 \mathrm{E}+00$ & $6.44 \mathrm{E}+00$ & $6.44 \mathrm{E}+00$ & $6.44 \mathrm{E}+00$ \\
\hline $\mathrm{Al}(\mathrm{OH}) 4$ & 3.20E-01 & $3.32 \mathrm{E}-01$ & $3.20 \mathrm{E}-01$ & $4.00 \mathrm{E}-01$ & $4.01 \mathrm{E}-01$ & $3.19 \mathrm{E}-01$ & 3.17E-01 & $3.84 \mathrm{E}-01$ & 3.93E-01 & $4.01 \mathrm{E}-01$ & $3.91 \mathrm{E}-01$ & $3.15 \mathrm{E}-01$ & $3.13 \mathrm{E}-01$ & $3.08 \mathrm{E}-01$ & $3.22 \mathrm{E}-01$ \\
\hline $\mathrm{CO} 3$ & $2.18 \mathrm{E}-01$ & $2.17 \mathrm{E}-01$ & $2.16 \mathrm{E}-01$ & $1.32 \mathrm{E}-01$ & $1.30 \mathrm{E}-01$ & $2.22 \mathrm{E}-01$ & $2.27 \mathrm{E}-01$ & $1.30 \mathrm{E}-01$ & $1.30 \mathrm{E}-01$ & $1.35 \mathrm{E}-01$ & $1.35 \mathrm{E}-01$ & $2.18 \mathrm{E}-01$ & $2.20 \mathrm{E}-01$ & $2.13 \mathrm{E}-01$ & $2.24 \mathrm{E}-01$ \\
\hline$\overline{\mathrm{C2O}}$ & $2.18 \mathrm{E}-02$ & $2.17 \mathrm{E}-02$ & $2.18 \mathrm{E}-02$ & $1.41 \mathrm{E}-02$ & $1.38 \mathrm{E}-02$ & $2.24 \mathrm{E}-02$ & $2.19 \mathrm{E}-02$ & $1.38 \mathrm{E}-02$ & $1.40 \mathrm{E}-02$ & $1.43 \mathrm{E}-02$ & $1.39 \mathrm{E}-02$ & $2.17 \mathrm{E}-02$ & $2.17 \mathrm{E}-02$ & $2.16 \mathrm{E}-02$ & $2.21 \mathrm{E}-02$ \\
\hline NO2 & $1.26 \mathrm{E}-01$ & $1.28 \mathrm{E}-01$ & $1.35 \mathrm{E}-01$ & $7.48 \mathrm{E}-01$ & $7.62 \mathrm{E}-01$ & $1.02 \mathrm{E}-01$ & $1.24 \mathrm{E}-01$ & 7.57E-01 & $7.52 \mathrm{E}-01$ & $7.45 \mathrm{E}-01$ & $7.39 \mathrm{E}-01$ & $1.30 \mathrm{E}-01$ & $1.34 \mathrm{E}-01$ & $1.31 \mathrm{E}-01$ & $1.13 \mathrm{E}-01$ \\
\hline NO3 & $3.72 \mathrm{E}+00$ & $3.71 \mathrm{E}+00$ & $3.73 \mathrm{E}+00$ & $2.65 \mathrm{E}+00$ & $2.61 \mathrm{E}+00$ & $3.83 \mathrm{E}+00$ & $3.76 \mathrm{E}+00$ & $2.60 \mathrm{E}+00$ & $2.61 \mathrm{E}+00$ & $2.67 \mathrm{E}+00$ & $2.65 \mathrm{E}+00$ & $3.69 \mathrm{E}+00$ & $3.70 \mathrm{E}+00$ & $3.69 \mathrm{E}+00$ & $3.78 \mathrm{E}+00$ \\
\hline$\overline{\mathrm{OH}}$ & $1.07 \mathrm{E}+00$ & $1.07 \mathrm{E}+00$ & $1.02 \mathrm{E}+00$ & $1.86 \mathrm{E}+00$ & $1.88 \mathrm{E}+00$ & $9.25 \mathrm{E}-01$ & +00 & $1.98 \mathrm{E}+00$ & $1.91 \mathrm{E}+00$ & $1.87 \mathrm{E}+00$ & $1.87 \mathrm{E}+00$ & $1.12 \mathrm{E}+00$ & $1.15 \mathrm{E}+00$ & $9.99 \mathrm{E}-01$ & $9.63 \mathrm{E}-01$ \\
\hline PO4 & $1.64 \mathrm{E}-03$ & $1.63 \mathrm{E}-03$ & $1.77 \mathrm{E}-03$ & $1.56 \mathrm{E}-02$ & $1.55 \mathrm{E}-02$ & $1.41 \mathrm{E}-03$ & $1.41 \mathrm{E}-03$ & $1.57 \mathrm{E}-02$ & $1.54 \mathrm{E}-02$ & $1.58 \mathrm{E}-02$ & $1.58 \mathrm{E}-02$ & $1.50 \mathrm{E}-03$ & $53 \mathrm{E}-03$ & $1.61 \mathrm{E}-03$ & $1.40 \mathrm{E}-03$ \\
\hline SO4 & $3.17 \mathrm{E}-01$ & $3.16 \mathrm{E}-01$ & $3.16 \mathrm{E}-01$ & $1.82 \mathrm{E}-01$ & $1.78 \mathrm{E}-01$ & $3.27 \mathrm{E}-01$ & $3.18 \mathrm{E}-01$ & $1.78 \mathrm{E}-01$ & $1.78 \mathrm{E}-01$ & $1.84 \mathrm{E}-01$ & $1.80 \mathrm{E}-01$ & $3.14 \mathrm{E}-01$ & $3.15 \mathrm{E}-01$ & $3.14 \mathrm{E}-01$ & $3.21 \mathrm{E}-01$ \\
\hline$\overline{\mathrm{Ag}}$ & $1.79 \mathrm{E}-08$ & $1.05 \mathrm{E}-07$ & $7.74 \mathrm{E}-08$ & $3.95 \mathrm{E}-08$ & $3.34 \mathrm{E}-08$ & $3.73 \mathrm{E}-08$ & $3.63 \mathrm{E}-08$ & $3.34 \mathrm{E}-08$ & $3.34 \mathrm{E}-08$ & $4.38 \mathrm{E}-08$ & $3.37 \mathrm{E}-08$ & $3.59 \mathrm{E}-08$ & $3.60 \mathrm{E}-08$ & $3.58 \mathrm{E}-08$ & $3.66 \mathrm{E}-08$ \\
\hline As & $3.15 \mathrm{E}-06$ & $2.85 \mathrm{E}-06$ & $3.72 \mathrm{E}-06$ & $5.15 \mathrm{E}-06$ & $5.26 \mathrm{E}-05$ & $2.57 \mathrm{E}-05$ & E-05 & $9.14 \mathrm{E}-05$ & $6.58 \mathrm{E}-05$ & $3.56 \mathrm{E}-06$ & $4.36 \mathrm{E}-05$ & $1.09 \mathrm{E}-04$ & $1.20 \mathrm{E}-04$ & $6.56 \mathrm{E}-05$ & $4.58 \mathrm{E}-05$ \\
\hline$\overline{\mathrm{Ba}}$ & $3.12 \mathrm{E}-09$ & $3.11 \mathrm{E}-08$ & $2.34 \mathrm{E}-08$ & $2.07 \mathrm{E}-07$ & -- & --- & ---- & -- & -- & $7.47 \mathrm{E}-09$ & --- & --- & --- & --- & 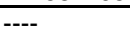 \\
\hline $\mathrm{Ca}$ & $4.92 \mathrm{E}-05$ & $4.90 \mathrm{E}-05$ & $4.91 \mathrm{E}-05$ & $3.19 \mathrm{E}-05$ & $3.01 \mathrm{E}-05$ & $5.22 \mathrm{E}-05$ & $4.93 \mathrm{E}-05$ & $3.01 \mathrm{E}-05$ & $3.01 \mathrm{E}-05$ & $3.24 \mathrm{E}-05$ & 3.03E-01 & $4.88 \mathrm{E}-05$ & $4.89 \mathrm{E}-05$ & 4.87E-05 & $5.00 \mathrm{E}-05$ \\
\hline$\overline{C d}$ & $8.59 \mathrm{E}-08$ & $8.18 \mathrm{E}-08$ & $1.09 \mathrm{E}-07$ & $1.35 \mathrm{E}-07$ & $1.42 \mathrm{E}-06$ & $3.08 \mathrm{E}-07$ & $3 \mathrm{E}-06$ & $2.92 \mathrm{E}-06$ & $3 \mathrm{E}-06$ & $7.30 \mathrm{E}-08$ & $1.06 \mathrm{E}-06$ & $3.55 \mathrm{E}-06$ & $3.95 \mathrm{E}-06$ & $1.88 \mathrm{E}-06$ & $1.10 \mathrm{E}-06$ \\
\hline$\overline{\mathrm{Cl}}$ & $4.19 \mathrm{E}-02$ & $4.18 \mathrm{E}-02$ & $4.18 \mathrm{E}-02$ & $3.83 \mathrm{E}-02$ & 3.74E-02 & $4.28 \mathrm{E}-02$ & E-02 & 3.76E-02 & E-02 & $3.86 \mathrm{E}-02$ & $3.78 \mathrm{E}-02$ & $4.17 \mathrm{E}-02$ & $4.19 \mathrm{E}-02$ & $4.15 \mathrm{E}-02$ & $4.21 \mathrm{E}-02$ \\
\hline Co & $4.57 \mathrm{E}-07$ & $4.66 \mathrm{E}-07$ & $3.84 \mathrm{E}-07$ & $2.05 \mathrm{E}-07$ & $3.00 \mathrm{E}-07$ & $1.98 \mathrm{E}-07$ & $7 \mathrm{E}-07$ & $4.60 \mathrm{E}-07$ & 3.54E-07 & $1.99 \mathrm{E}-07$ & $2.63 \mathrm{E}-07$ & 5.39E-07 & $5.84 \mathrm{E}-07$ & $3.60 \mathrm{E}-07$ & $2.79 \mathrm{E}-07$ \\
\hline $\mathrm{Cr}$ & $1.90 \mathrm{E}-05$ & $1.15 \mathrm{E}-05$ & $3.83 \mathrm{E}-05$ & $1.59 \mathrm{E}-04$ & $2.10 \mathrm{E}-04$ & $2.61 \mathrm{E}-04$ & E-04 & $1.79 \mathrm{E}-04$ & $2.00 \mathrm{E}-04$ & $2.01 \mathrm{E}-04$ & DE-04 & $1.83 \mathrm{E}-04$ & $6 \mathrm{E}-04$ & $2.18 \mathrm{E}-04$ & $40 \mathrm{E}-04$ \\
\hline $\mathrm{Cu}$ & $1.64 \mathrm{E}-04$ & $1.69 \mathrm{E}-04$ & $1.24 \mathrm{E}-04$ & $2.99 \mathrm{E}-05$ & $8.41 \mathrm{E}-05$ & $1.84 \mathrm{E}-05$ & $1.09 \mathrm{E}-04$ & $1.73 \mathrm{E}-04$ & $1.14 \mathrm{E}-04$ & $2.56 \mathrm{E}-05$ & $6.31 \mathrm{E}-05$ & $2.10 \mathrm{E}-04$ & $2.34 \mathrm{E}-04$ & $1.11 \mathrm{E}-04$ & $6.50 \mathrm{E}-05$ \\
\hline$\overline{F e}$ & $1.38 \mathrm{E}-06$ & $1.38 \mathrm{E}-06$ & $1.07 \mathrm{E}-06$ & $8.36 \mathrm{E}-07$ & $3.83 \mathrm{E}-06$ & $9.41 \mathrm{E}-07$ & $4.92 \mathrm{E}-06$ & 7.74E-06 & $5.15 \mathrm{E}-06$ & $1.95 \mathrm{E}-06$ & $2.90 \mathrm{E}-06$ & $9.40 \mathrm{E}-06$ & $1.05 \mathrm{E}-05$ & $5.04 \mathrm{E}-06$ & $3.00 \mathrm{E}-06$ \\
\hline $\bar{F}$ & $6.95 \mathrm{E}-02$ & $6.93 \mathrm{E}-02$ & $6.94 \mathrm{E}-02$ & $3.59 \mathrm{E}-02$ & $2 \mathrm{E}-02$ & $7.15 \mathrm{E}-02$ & & $3.52 \mathrm{E}-02$ & E-02 & 3E-02 & $3 \mathrm{E}-02$ & $6.90 \mathrm{E}-02$ & $6.92 \mathrm{E}-02$ & $6.87 \mathrm{E}-02$ & $7.03 \mathrm{E}-02$ \\
\hline $\mathrm{Hg}$ & $1.07 \mathrm{E}-06$ & $1.07 \mathrm{E}-06$ & $1.07 \mathrm{E}-06$ & $2.02 \mathrm{E}-06$ & $1.21 \mathrm{E}-05$ & $1.29 \mathrm{E}-05$ & E-05 & $1.29 \mathrm{E}-05$ & $1.24 \mathrm{E}-05$ & $1.02 \mathrm{E}-06$ & $1.20 \mathrm{E}-05$ & $1.41 \mathrm{E}-05$ & $1.44 \mathrm{E}-05$ & $1.32 \mathrm{E}-05$ & $1.31 \mathrm{E}-05$ \\
\hline $\mathrm{K}$ & $7.45 \mathrm{E}-03$ & $7.64 \mathrm{E}-03$ & $7.44 \mathrm{E}-03$ & $5.24 \mathrm{E}-03$ & $5.81 \mathrm{E}-03$ & $5.09 \mathrm{E}-03$ & E-03 & 7.67E-03 & $6.03 \mathrm{E}-03$ & $4.73 \mathrm{E}-03$ & $5.08 \mathrm{E}-03$ & $8.04 \mathrm{E}-03$ & $8.48 \mathrm{E}-03$ & $6.44 \mathrm{E}-03$ & $5.89 \mathrm{E}-03$ \\
\hline$\overline{M g}$ & --- & --- & $1.01 \mathrm{E}-08$ & $1.31 \mathrm{E}-07$ & $5.17 \mathrm{E}-08$ & $1.62 \mathrm{E}-07$ & $\mathrm{E}-08$ & $\ldots$ & E-08 & $1.38 \mathrm{E}-07$ & $8.20 \mathrm{E}-08$ & --- & --- & $2.62 \mathrm{E}-08$ & $9.39 \mathrm{E}-08$ \\
\hline$M n$ & $5.86 \mathrm{E}-08$ & $7.00 \mathrm{E}-08$ & $9.36 \mathrm{E}-08$ & E-07 & $1.98 \mathrm{E}-07$ & पE-07 & $2.42 \mathrm{E}-07$ & 3.35E-07 & $2.44 \mathrm{E}-07$ & -08 & -07 & $3.98 \mathrm{E}-07$ & $4.35 \mathrm{E}-07$ & $2.45 \mathrm{E}-07$ & $5 \mathrm{E}-07$ \\
\hline Nd & --- & $-\cdots$ & --- & $4.34 \mathrm{E}-06$ & --- & $5.85 \mathrm{E}-06$ & 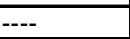 & 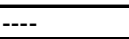 & --- & $4.75 \mathrm{E}-06$ & $1.59 \mathrm{E}-06$ & -- & --- & --- & $2.00 \mathrm{E}-06$ \\
\hline$\overline{\mathrm{Ni}}$ & $1.10 \mathrm{E}-06$ & $1.12 \mathrm{E}-06$ & $9.01 \mathrm{E}-07$ & $4.30 \mathrm{E}-07$ & $6.85 \mathrm{E}-07$ & $4.00 \mathrm{E}-07$ & $8.28 \mathrm{E}-07$ & $1.11 \mathrm{E}-06$ & $8.30 \mathrm{E}-07$ & $4.13 \mathrm{E}-07$ & $5.87 \mathrm{E}-07$ & $1.32 \mathrm{E}-06$ & $1.43 \mathrm{E}-06$ & $8.37 \mathrm{E}-07$ & $6.20 \mathrm{E}-07$ \\
\hline $\mathbf{P b}$ & $3.00 \mathrm{E}-06$ & $2.48 \mathrm{E}-06$ & $3.41 \mathrm{E}-06$ & $5.00 \mathrm{E}-06$ & ---- & $-\overline{-}_{-1}$ & --- & --- & --- & $2.97 \mathrm{E}-06$ & --- & --- & --- & -- & --- \\
\hline$\overline{R u}$ & $1.73 \mathrm{E}-05$ & $1.78 \mathrm{E}-05$ & $1.34 \mathrm{E}-05$ & $4.15 \mathrm{E}-06$ & $9.41 \mathrm{E}-06$ & $3.14 \mathrm{E}-06$ & $1.19 \mathrm{E}-05$ & $1.81 \mathrm{E}-05$ & $1.23 \mathrm{E}-05$ & $3.76 \mathrm{E}-06$ & $7.38 \mathrm{E}-06$ & $2.18 \mathrm{E}-05$ & $2.42 \mathrm{E}-05$ & $1.22 \mathrm{E}-05$ & $7.67 \mathrm{E}-06$ \\
\hline $\mathrm{Se}$ & $2.23 \mathrm{E}-05$ & $1.71 \mathrm{E}-05$ & $2.87 \mathrm{E}-05$ & $6.04 \mathrm{E}-05$ & $1.62 \mathrm{E}-04$ & $2.18 \mathrm{E}-04$ & $1.67 \mathrm{E}-04$ & $1.17 \mathrm{E}-04$ & $1.46 \mathrm{E}-04$ & $5.85 \mathrm{E}-05$ & $1.74 \mathrm{E}-04$ & $1.13 \mathrm{E}-04$ & $1.02 \mathrm{E}-04$ & $1.62 \mathrm{E}-04$ & $1.90 \mathrm{E}-04$ \\
\hline$\overline{\mathrm{Si}}$ & $1.06 \mathrm{E}-04$ & $9.29 \mathrm{E}-05$ & $5.44 \mathrm{E}-04$ & $1.81 \mathrm{E}-05$ & ---- & -- & ---- & --- & ---- & $1.42 \mathrm{E}-04$ & --.- & -.- & ---- & ---- & --- \\
\hline Sr & $\begin{array}{ll}--- \\
\end{array}$ & ---- & $\begin{array}{ll}--- \\
\end{array}$ & $2.92 \mathrm{E}-06$ & --- & $4.05 \mathrm{E}-06$ & --- & --- & --- & $3.23 \mathrm{E}-06$ & $7.95 \mathrm{E}-07$ & --- & --- & --- & $1.08 \mathrm{E}-06$ \\
\hline$Z \mathbf{n}$ & $1.99 \mathrm{E}-04$ & $2.05 \mathrm{E}-04$ & \begin{tabular}{|c|}
$1.56 \mathrm{E}-04$ \\
\end{tabular} & $5.24 \mathrm{E}-05$ & $1.11 \mathrm{E}-04$ & $4.16 \mathrm{E}-05$ & $1.39 \mathrm{E}-04$ & $2.07 \mathrm{E}-04$ & $1.43 \mathrm{E}-04$ & $4.80 \mathrm{E}-05$ & $8.82 \mathrm{E}-05$ & $2.49 \mathrm{E}-04$ & $2.76 \mathrm{E}-04$ & 1.42E-04 & $9.19 \mathrm{E}-05$ \\
\hline$\overline{Z r}$ & $5.25 \mathrm{E}-06$ & $5.41 \mathrm{E}-06$ & $4.00 \mathrm{E}-06$ & $1.06 \mathrm{E}-06$ & $2.74 \mathrm{E}-06$ & $7.12 \mathrm{E}-07$ & $3.52 \mathrm{E}-06$ & $5.50 \mathrm{E}-06$ & $3.68 \mathrm{E}-06$ & $9.28 \mathrm{E}-07$ & $2.09 \mathrm{E}-06$ & $6.68 \mathrm{E}-06$ & $7.43 \mathrm{E}-06$ & $3.60 \mathrm{E}-06$ & $2.16 \mathrm{E}-06$ \\
\hline Insol.Sol.mg/L & 100 & 100 & 100 & 100 & 100 & 100 & 100 & 100 & 100 & 100 & 100 & 10 & 100 & 100 & 100 \\
\hline
\end{tabular}


Table 6: Projected Feed Composition After Dissolution and Dilution to 6.44 M Total Sodium Concentration $100 \mathrm{mg} / \mathrm{L}$ sludge; $30 \%$ of interstitial supernate remaining (continued)

\begin{tabular}{|c|c|c|c|c|c|c|c|c|c|c|c|c|c|c|c|}
\hline \multicolumn{16}{|c|}{ Radionuclide Composition (Ci/gal) } \\
\hline & Tank 25 & Tank 27 & Tank 28 & Tank 29 & Tank 31 & Tank 33 & Tank 34 & \begin{tabular}{|l|} 
Tank 36 \\
\end{tabular} & Tank 37 & Tank 38 & Tank 41 & Tank 44 & Tank 45 & Tank 46 & Tank 47 \\
\hline C-14 & $5.62 \mathrm{E}-10$ & $5.62 \mathrm{E}-10$ & $5.62 \mathrm{E}-10$ & $5.62 \mathrm{E}-10$ & $5.62 \mathrm{E}-10$ & $5.62 \mathrm{E}-10$ & $5.62 \mathrm{E}-10$ & $5.62 \mathrm{E}-10$ & $5.62 \mathrm{E}-10$ & $5.62 \mathrm{E}-10$ & $5.62 \mathrm{E}-10$ & $5.62 \mathrm{E}-10$ & $5.62 \mathrm{E}-10$ & $5.62 \mathrm{E}-10$ & $5.62 \mathrm{E}-10$ \\
\hline Ni-59 & $3.23 \mathrm{E}-07$ & $3.23 \mathrm{E}-07$ & $3.23 \mathrm{E}-07$ & $3.23 \mathrm{E}-07$ & $3.23 \mathrm{E}-07$ & $3.23 \mathrm{E}-07$ & $3.23 \mathrm{E}-07$ & $3.23 \mathrm{E}-07$ & $3.23 \mathrm{E}-07$ & $3.23 \mathrm{E}-07$ & $3.23 \mathrm{E}-07$ & $3.23 \mathrm{E}-07$ & $3.23 \mathrm{E}-07$ & $3.23 \mathrm{E}-07$ & $3.23 \mathrm{E}-07$ \\
\hline $\mathrm{Ni}-63$ & $5.02 \mathrm{E}-10$ & $5.02 \mathrm{E}-10$ & $5.02 \mathrm{E}-10$ & $5.02 \mathrm{E}-10$ & $5.02 \mathrm{E}-10$ & $5.02 \mathrm{E}-10$ & $5.02 \mathrm{E}-10$ & $5.02 \mathrm{E}-10$ & $5.02 \mathrm{E}-10$ & $5.02 \mathrm{E}-10$ & $5.02 \mathrm{E}-10$ & $5.02 \mathrm{E}-10$ & $5.02 \mathrm{E}-10$ & $5.02 \mathrm{E}-10$ & $5.02 \mathrm{E}-10$ \\
\hline Co-60 & $5.14 \mathrm{E}-05$ & $5.14 \mathrm{E}-05$ & $5.14 \mathrm{E}-05$ & $5.14 \mathrm{E}-05$ & $5.14 \mathrm{E}-05$ & $5.14 \mathrm{E}-05$ & $5.14 \mathrm{E}-05$ & $5.14 \mathrm{E}-05$ & $5.14 \mathrm{E}-05$ & $5.14 \mathrm{E}-05$ & $5.14 \mathrm{E}-05$ & $5.14 \mathrm{E}-05$ & $5.14 \mathrm{E}-05$ & $5.14 \mathrm{E}-05$ & $5.14 \mathrm{E}-05$ \\
\hline Se-79 & $1.91 \mathrm{E}-07$ & $1.91 \mathrm{E}-07$ & $1.91 \mathrm{E}-07$ & $1.91 \mathrm{E}-07$ & $1.91 \mathrm{E}-07$ & $1.91 \mathrm{E}-07$ & $1.91 \mathrm{E}-07$ & $1.91 \mathrm{E}-07$ & $1.91 \mathrm{E}-07$ & $1.91 \mathrm{E}-07$ & $1.91 \mathrm{E}-07$ & $1.91 \mathrm{E}-07$ & $1.91 \mathrm{E}-07$ & $1.91 \mathrm{E}-07$ & $1.91 \mathrm{E}-07$ \\
\hline Tc-99 & $3.24 \mathrm{E}-06$ & $3.24 \mathrm{E}-06$ & $3.24 \mathrm{E}-06$ & $3.24 \mathrm{E}-06$ & $3.24 \mathrm{E}-06$ & $3.24 \mathrm{E}-06$ & $3.24 \mathrm{E}-06$ & $3.24 \mathrm{E}-06$ & $3.24 \mathrm{E}-06$ & $3.24 \mathrm{E}-06$ & $3.24 \mathrm{E}-06$ & $3.24 \mathrm{E}-06$ & $3.24 \mathrm{E}-06$ & $3.24 \mathrm{E}-06$ & $3.24 \mathrm{E}-06$ \\
\hline Ru-106 & $1.20 \mathrm{E}-06$ & $1.20 \mathrm{E}-06$ & $1.20 \mathrm{E}-06$ & $1.20 \mathrm{E}-06$ & $1.20 \mathrm{E}-06$ & $1.20 \mathrm{E}-06$ & $1.20 \mathrm{E}-06$ & $1.20 \mathrm{E}-06$ & $1.20 \mathrm{E}-06$ & $1.20 \mathrm{E}-06$ & $1.20 \mathrm{E}-06$ & $1.20 \mathrm{E}-06$ & $1.20 \mathrm{E}-06$ & $1.20 \mathrm{E}-06$ & $1.20 \mathrm{E}-06$ \\
\hline Rh-106 & $1.20 \mathrm{E}-06$ & $1.20 \mathrm{E}-06$ & $1.20 \mathrm{E}-06$ & $1.20 \mathrm{E}-06$ & $1.20 \mathrm{E}-06$ & $1.20 \mathrm{E}-06$ & $1.20 \mathrm{E}-06$ & $1.20 \mathrm{E}-06$ & $1.20 \mathrm{E}-06$ & $1.20 \mathrm{E}-06$ & $1.20 \mathrm{E}-06$ & $1.20 \mathrm{E}-06$ & $1.20 \mathrm{E}-06$ & $1.20 \mathrm{E}-06$ & $1.20 \mathrm{E}-06$ \\
\hline Sb-125 & $3.07 \mathrm{E}-05$ & $3.07 \mathrm{E}-05$ & $3.07 \mathrm{E}-05$ & $3.07 \mathrm{E}-05$ & $3.07 \mathrm{E}-05$ & $3.07 \mathrm{E}-05$ & $3.07 \mathrm{E}-05$ & $3.07 \mathrm{E}-05$ & 3.07E-05 & $3.07 \mathrm{E}-05$ & 3.07E-05 & 3.07E-05 & $3.07 \mathrm{E}-05$ & $3.07 \mathrm{E}-05$ & 3.07E-05 \\
\hline Sn-126 & $2.55 \mathrm{E}-07$ & $2.55 \mathrm{E}-07$ & $2.55 \mathrm{E}-07$ & $2.55 \mathrm{E}-07$ & $2.55 \mathrm{E}-07$ & $2.55 \mathrm{E}-07$ & $2.55 \mathrm{E}-07$ & $2.55 \mathrm{E}-07$ & $2.55 \mathrm{E}-07$ & $2.55 \mathrm{E}-07$ & $2.55 \mathrm{E}-07$ & $2.55 \mathrm{E}-07$ & $2.55 \mathrm{E}-07$ & $2.55 \mathrm{E}-07$ & $2.55 \mathrm{E}-07$ \\
\hline I-129 & $2.98 \mathrm{E}-11$ & $2.98 \mathrm{E}-11$ & $2.98 \mathrm{E}-11$ & $2.98 \mathrm{E}-11$ & $2.98 \mathrm{E}-11$ & $2.98 \mathrm{E}-11$ & $2.98 \mathrm{E}-11$ & $2.98 \mathrm{E}-11$ & $2.98 \mathrm{E}-11$ & $2.98 \mathrm{E}-11$ & $2.98 \mathrm{E}-11$ & $2.98 \mathrm{E}-11$ & $2.98 \mathrm{E}-11$ & $2.98 \mathrm{E}-11$ & $2.98 \mathrm{E}-11$ \\
\hline$\overline{C s-134}$ & $1.34 \mathrm{E}-06$ & $1.34 \mathrm{E}-06$ & $1.34 \mathrm{E}-06$ & $1.34 \mathrm{E}-06$ & $1.34 \mathrm{E}-06$ & $4 \mathrm{E}-06$ & $1.34 \mathrm{E}-06$ & $1.34 \mathrm{E}-06$ & $1.34 \mathrm{E}-06$ & $1.34 \mathrm{E}-06$ & $1.34 \mathrm{E}-06$ & $1.34 \mathrm{E}-06$ & $1.34 \mathrm{E}-06$ & $1.34 \mathrm{E}-06$ & $1.34 \mathrm{E}-06$ \\
\hline Cs-135 & $2.28 \mathrm{E}-09$ & $2.28 \mathrm{E}-09$ & $2.28 \mathrm{E}-09$ & $2.28 \mathrm{E}-09$ & $2.28 \mathrm{E}-09$ & $8 \mathrm{E}-09$ & 2.28 & $2.28 \mathrm{E}-09$ & $2.28 \mathrm{E}-09$ & $2.28 \mathrm{E}-09$ & $2.28 \mathrm{E}-09$ & $2.28 \mathrm{E}-09$ & $2.28 \mathrm{E}-09$ & $2.28 \mathrm{E}-09$ & $2.28 \mathrm{E}-09$ \\
\hline Cs-137 & $9.27 \mathrm{E}-02$ & $8.78 \mathrm{E}-02$ & $9.64 \mathrm{E}-02$ & $8.38 \mathrm{E}-02$ & $2.26 \mathrm{E}-01$ & $2.87 \mathrm{E}-03$ & $7.56 \mathrm{E}-02$ & $4.15 \mathrm{E}-01$ & $3.01 \mathrm{E}-01$ & $6.23 \mathrm{E}-03$ & $5.11 \mathrm{E}-02$ & $1.14 \mathrm{E}-01$ & $1.08 \mathrm{E}-01$ & $1.38 \mathrm{E}-01$ & $5.86 \mathrm{E}-02$ \\
\hline Ba-137m & $8.77 \mathrm{E}-02$ & $8.30 \mathrm{E}-02$ & $9.12 \mathrm{E}-02$ & $7.92 \mathrm{E}-02$ & $2.14 \mathrm{E}-01$ & $2.72 \mathrm{E}-03$ & $7.15 \mathrm{E}-02$ & $3.93 \mathrm{E}-01$ & $2.85 \mathrm{E}-01$ & $5.89 \mathrm{E}-03$ & $4.84 \mathrm{E}-02$ & 1.07E-01 & $1.02 \mathrm{E}-01$ & $1.31 \mathrm{E}-01$ & $5.54 \mathrm{E}-02$ \\
\hline Sr-90 & $1.13 \mathrm{E}-02$ & $1.13 \mathrm{E}-02$ & $1.13 \mathrm{E}-02$ & $1.14 \mathrm{E}-02$ & $1.14 \mathrm{E}-02$ & $\mathrm{E}-02$ & 1.15 & $1.14 \mathrm{E}-02$ & $1.14 \mathrm{E}-02$ & $\mathrm{E}-02$ & $1.13 \mathrm{E}-02$ & $1.13 \mathrm{E}-02$ & $3 \mathrm{E}-02$ & $1.13 \mathrm{E}-02$ & $1.13 \mathrm{E}-02$ \\
\hline$Y-90$ & $1.13 \mathrm{E}-02$ & $\mathrm{E}-02$ & $1.13 \mathrm{E}-02$ & $1.14 \mathrm{E}-02$ & $1.14 \mathrm{E}-02$ & $\mathrm{E}-02$ & 1.15 & $1.14 \mathrm{E}-02$ & $1.14 \mathrm{E}-02$ & $1.13 \mathrm{E}-02$ & $1.13 \mathrm{E}-02$ & $1.13 \mathrm{E}-02$ & $1.13 \mathrm{E}-02$ & $1.13 \mathrm{E}-02$ & $1.13 \mathrm{E}-02$ \\
\hline $\mathrm{H}-3$ & \begin{tabular}{|l|}
---- \\
\end{tabular} & ---- & \begin{tabular}{|l|}
---- \\
\end{tabular} & \begin{tabular}{|l|}
---- \\
\end{tabular} & \begin{tabular}{|l|}
---- \\
\end{tabular} & ---- & 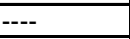 & \begin{tabular}{|l|}
---- \\
\end{tabular} & \begin{tabular}{|l|}
--- \\
\end{tabular} & \begin{tabular}{|l|}
---- \\
\end{tabular} & $\begin{array}{ll}--- \\
\end{array}$ & \begin{tabular}{|l|}
---- \\
\end{tabular} & ---- & $\begin{array}{ll}--- \\
\end{array}$ & ---- \\
\hline Ce-144 & $\begin{array}{r}6.17 \mathrm{E}-07 \\
\end{array}$ & 6.17E-07 & \begin{tabular}{|r|}
$6.17 \mathrm{E}-07$ \\
\end{tabular} & 6.17E-07 & \begin{tabular}{|r|}
$6.17 \mathrm{E}-07$ \\
\end{tabular} & $6.17 \mathrm{E}-07$ & $6.17 \mathrm{E}-07$ & \begin{tabular}{|r|}
$6.17 \mathrm{E}-07$ \\
\end{tabular} & \begin{tabular}{|r}
$6.17 \mathrm{E}-07$ \\
\end{tabular} & \begin{tabular}{|r|}
$6.17 \mathrm{E}-07$ \\
\end{tabular} & \begin{tabular}{|r|}
$6.17 \mathrm{E}-07$ \\
\end{tabular} & \begin{tabular}{|r}
$6.17 \mathrm{E}-07$ \\
\end{tabular} & \begin{tabular}{|r|}
$6.17 \mathrm{E}-07$ \\
\end{tabular} & \begin{tabular}{|r|}
$6.17 \mathrm{E}-07$ \\
\end{tabular} & \begin{tabular}{|c|}
$6.17 \mathrm{E}-07$ \\
\end{tabular} \\
\hline Pr-144 & $6.17 \mathrm{E}-07$ & 6.1 & E-07 & E-07 & E-07 & -07 & & E-07 & $\mathrm{E}-07$ & -07 & E-07 & E-07 & $\mathrm{E}-07$ & EE-07 & 7E-07 \\
\hline Pm-147 & $6.46 \mathrm{E}-04$ & 6.46 & $6.46 \mathrm{E}-04$ & $6.46 \mathrm{E}-04$ & $6.46 \mathrm{E}-04$ & E-04 & 6.46 & $6.46 \mathrm{E}-04$ & E-04 & 6E-04 & E-04 & $6 \mathrm{E}-04$ & $6.46 \mathrm{E}-04$ & $6.46 \mathrm{E}-04$ & $6.46 \mathrm{E}-04$ \\
\hline Eu-154 & $1.26 \mathrm{E}-04$ & $1.26 \mathrm{E}-04$ & $1.26 \mathrm{E}-04$ & $1.26 \mathrm{E}-04$ & $1.26 \mathrm{E}-04$ & $1.26 \mathrm{E}-04$ & 1.26 & $1.26 \mathrm{E}-04$ & $1.26 \mathrm{E}-04$ & $6 \mathrm{E}-04$ & $6 \mathrm{E}-04$ & $6 \mathrm{E}-04$ & $6 \mathrm{E}-04$ & $1.26 \mathrm{E}-04$ & $1.26 \mathrm{E}-04$ \\
\hline Th-232 & $3.62 \mathrm{E}-10$ & $3.62 \mathrm{E}-10$ & $3.62 \mathrm{E}-10$ & $3.73 \mathrm{E}-10$ & $3.72 \mathrm{E}-10$ & $3.62 \mathrm{E}-10$ & $3.62 \mathrm{E}-10$ & $3.72 \mathrm{E}-10$ & $3.72 \mathrm{E}-10$ & $3.62 \mathrm{E}-10$ & $3.62 \mathrm{E}-10$ & $3.62 \mathrm{E}-10$ & $3.62 \mathrm{E}-10$ & $3.62 \mathrm{E}-10$ & $3.62 \mathrm{E}-10$ \\
\hline $\mathrm{U}-232$ & $1.53 \mathrm{E}-10$ & $1.52 \mathrm{E}-10$ & $1.53 \mathrm{E}-10$ & $8.12 \mathrm{E}-11$ & $8.11 \mathrm{E}-11$ & $\mathrm{E}-09$ & & $8.11 \mathrm{E}-11$ & $1 \mathrm{E}-11$ & $\mathrm{E}-11$ & $0 \mathrm{E}-11$ & $1.52 \mathrm{E}-10$ & $2 \mathrm{E}-10$ & $1.52 \mathrm{E}-10$ & $1.54 \mathrm{E}-10$ \\
\hline U-233 & $1.22 \mathrm{E}-08$ & $1.22 \mathrm{E}-08$ & $1.22 \mathrm{E}-08$ & $1.45 \mathrm{E}-08$ & $1.44 \mathrm{E}-08$ & $1.22 \mathrm{E}-08$ & 1.22 & $1.44 \mathrm{E}-08$ & $1.44 \mathrm{E}-08$ & $1.22 \mathrm{E}-08$ & $1.22 \mathrm{E}-08$ & $1.22 \mathrm{E}-08$ & $1.22 \mathrm{E}-08$ & $1.22 \mathrm{E}-08$ & $1.22 \mathrm{E}-08$ \\
\hline U-234 & $3.92 \mathrm{E}-09$ & $3.92 \mathrm{E}-09$ & $3.92 \mathrm{E}-09$ & $4.23 \mathrm{E}-09$ & $4.23 \mathrm{E}-09$ & $2 \mathrm{E}-09$ & $3.92 \mathrm{E}-09$ & $4.23 \mathrm{E}-09$ & EE-09 & $4 \mathrm{E}-09$ & E-09 & $3.92 \mathrm{E}-09$ & 3.92E-09 & $3.92 \mathrm{E}-09$ & $3.92 \mathrm{E}-09$ \\
\hline $\mathrm{U}-235$ & $3.61 \mathrm{E}-10$ & $3.60 \mathrm{E}-10$ & $3.60 \mathrm{E}-10$ & $2.02 \mathrm{E}-10$ & $2.02 \mathrm{E}-10$ & $1.40 \mathrm{E}-09$ & $1.13 \mathrm{E}-09$ & $2.02 \mathrm{E}-10$ & $2.02 \mathrm{E}-10$ & $2.46 \mathrm{E}-10$ & $2.45 \mathrm{E}-10$ & $3.59 \mathrm{E}-10$ & $3.60 \mathrm{E}-10$ & $3.59 \mathrm{E}-10$ & $3.62 \mathrm{E}-10$ \\
\hline $\mathrm{U}-236$ & $5.56 \mathrm{E}-10$ & $5.56 \mathrm{E}-10$ & $5.56 \mathrm{E}-10$ & $5.89 \mathrm{E}-10$ & $5.88 \mathrm{E}-10$ & $5.56 \mathrm{E}-10$ & $5.56 \mathrm{E}-10$ & $5.88 \mathrm{E}-10$ & $5.88 \mathrm{E}-10$ & $\mathrm{E}-09$ & $1.33 \mathrm{E}-09$ & $5.56 \mathrm{E}-10$ & E-10 & $5.56 \mathrm{E}-10$ & $5.56 \mathrm{E}-10$ \\
\hline U-238 & $2.12 \mathrm{E}-08$ & 2.12 & 2.12 & $E-09$ & E-09 & $\mathrm{E}-07$ & 7.23 & $6.04 \mathrm{E}-09$ & $6.04 \mathrm{E}-09$ & $5.94 \mathrm{E}-09$ & $E-09$ & $2.11 \mathrm{E}-08$ & $1 \mathrm{E}-08$ & $2.10 \mathrm{E}-08$ & $2.14 \mathrm{E}-08$ \\
\hline Np-237 & $8.47 \mathrm{E}-09$ & $8.47 \mathrm{E}-09$ & $8.47 \mathrm{E}-09$ & $9.28 \mathrm{E}-09$ & $9.26 \mathrm{E}-09$ & $9.90 \mathrm{E}-08$ & $5.97 \mathrm{E}-08$ & $9.26 \mathrm{E}-09$ & $9.26 \mathrm{E}-09$ & $1.30 \mathrm{E}-08$ & $1.29 \mathrm{E}-08$ & 8.47E-09 & 8.47E-09 & $8.47 \mathrm{E}-09$ & $8.47 \mathrm{E}-09$ \\
\hline Pu-238 & $3.65 \mathrm{E}-04$ & $3.65 \mathrm{E}-04$ & $3.65 \mathrm{E}-04$ & $2.42 \mathrm{E}-04$ & $2.42 \mathrm{E}-04$ & $2.38 \mathrm{E}-04$ & $2.38 \mathrm{E}-04$ & $2.42 \mathrm{E}-04$ & $2.42 \mathrm{E}-04$ & 3.82E-04 & $3.78 \mathrm{E}-04$ & $3.64 \mathrm{E}-04$ & 3.64E-04 & $3.64 \mathrm{E}-04$ & $3.66 \mathrm{E}-04$ \\
\hline Pu-239 & $2.22 \mathrm{E}-05$ & $2.21 \mathrm{E}-05$ & $2.21 \mathrm{E}-05$ & $4.18 \mathrm{E}-06$ & $4.18 \mathrm{E}-06$ & $1.73 \mathrm{E}-05$ & $1.48 \mathrm{E}-05$ & $4.18 \mathrm{E}-06$ & $4.18 \mathrm{E}-06$ & $4.54 \mathrm{E}-06$ & $4.53 \mathrm{E}-06$ & $2.20 \mathrm{E}-05$ & $2.21 \mathrm{E}-05$ & $2.20 \mathrm{E}-05$ & $2.24 \mathrm{E}-05$ \\
\hline Pu-240 & $5.95 \mathrm{E}-06$ & $5.93 \mathrm{E}-06$ & $5.94 \mathrm{E}-06$ & $1.94 \mathrm{E}-06$ & $1.94 \mathrm{E}-06$ & E-06 & $4.30 \mathrm{E}-06$ & $1.94 \mathrm{E}-06$ & $\mathrm{EE}-06$ & E-06 & $9 \mathrm{E}-06$ & $5.92 \mathrm{E}-06$ & $3 \mathrm{E}-06$ & $5.90 \mathrm{E}-06$ & $5.99 \mathrm{E}-06$ \\
\hline Pu-241 & $2.38 \mathrm{E}-04$ & $2.38 \mathrm{E}-04$ & $2.38 \mathrm{E}-04$ & $E-04$ & -04 & E-04 & 1.91 & E-04 & $E-04$ & E-04 & E-04 & $2.38 \mathrm{E}-04$ & $3 \mathrm{E}-04$ & $2.37 \mathrm{E}-04$ & $2.40 \mathrm{E}-04$ \\
\hline Pu-242 & $4.55 \mathrm{E}-09$ & $4.55 \mathrm{E}-09$ & $4.55 \mathrm{E}-09$ & $3.74 \mathrm{E}-09$ & $3.74 \mathrm{E}-09$ & $4.32 \mathrm{E}-09$ & $4.22 \mathrm{E}-09$ & $3.74 \mathrm{E}-09$ & $3.74 \mathrm{E}-09$ & $9.21 \mathrm{E}-09$ & $9.07 \mathrm{E}-09$ & $4.54 \mathrm{E}-09$ & $4.55 \mathrm{E}-09$ & $4.54 \mathrm{E}-09$ & $4.56 \mathrm{E}-09$ \\
\hline Am-241 & $2.69 \mathrm{E}-05$ & $2.69 \mathrm{E}-05$ & $2.69 \mathrm{E}-05$ & $2.78 \mathrm{E}-05$ & $2.78 \mathrm{E}-05$ & $1.65 \mathrm{E}-04$ & $1.64 \mathrm{E}-04$ & $2.78 \mathrm{E}-05$ & $2.78 \mathrm{E}-05$ & $2.77 \mathrm{E}-05$ & $2.77 \mathrm{E}-05$ & $2.69 \mathrm{E}-05$ & $2.69 \mathrm{E}-05$ & $2.69 \mathrm{E}-05$ & $2.69 \mathrm{E}-05$ \\
\hline$A m-242 m$ & $2.53 \mathrm{E}-08$ & $2.53 \mathrm{E}-08$ & $2.53 \mathrm{E}-08$ & $2.64 \mathrm{E}-08$ & $2.64 \mathrm{E}-08$ & $2.14 \mathrm{E}-07$ & $2.12 \mathrm{E}-07$ & $2.64 \mathrm{E}-08$ & $2.64 \mathrm{E}-08$ & $2.63 \mathrm{E}-08$ & $2.62 \mathrm{E}-08$ & $2.53 \mathrm{E}-08$ & $2.53 \mathrm{E}-08$ & $2.53 \mathrm{E}-08$ & $2.53 \mathrm{E}-08$ \\
\hline$\overline{\mathrm{Cm}-242}$ & & $-\cdots$ & 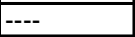 & --- & 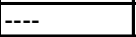 & 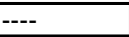 & 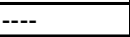 & 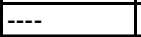 & 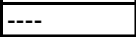 & ---- & 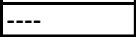 & 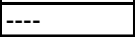 & 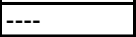 & $-\overline{--}$ & -- \\
\hline Cm-244 & $2.77 \mathrm{E}-05$ & $2.77 \mathrm{E}-05$ & $2.77 \mathrm{E}-05$ & $2.77 \mathrm{E}-05$ & $2.77 \mathrm{E}-05$ & $2.77 \mathrm{E}-05$ & $2.77 \mathrm{E}-05$ & $2.77 \mathrm{E}-05$ & $2.77 \mathrm{E}-05$ & $2.77 \mathrm{E}-05$ & $2.77 \mathrm{E}-05$ & $2.77 \mathrm{E}-05$ & $2.77 \mathrm{E}-05$ & $2.77 \mathrm{E}-05$ & $2.77 \mathrm{E}-05$ \\
\hline$\overline{C m-245}$ & $2.06 \mathrm{E}-09$ & $2.06 \mathrm{E}-09$ & $2.06 \mathrm{E}-09$ & $2.06 \mathrm{E}-09$ & $2.06 \mathrm{E}-09$ & $2.06 \mathrm{E}-09$ & $2.06 \mathrm{E}-09$ & $2.06 \mathrm{E}-09$ & $2.06 \mathrm{E}-09$ & $2.06 \mathrm{E}-09$ & $2.06 \mathrm{E}-09$ & $2.06 \mathrm{E}-09$ & $2.06 \mathrm{E}-09$ & $2.06 \mathrm{E}-09$ & $2.06 \mathrm{E}-09$ \\
\hline Total Alpha & $4.48 \mathrm{E}-04$ & $4.47 \mathrm{E}-04$ & $4.48 \mathrm{E}-04$ & $3.03 \mathrm{E}-04$ & $3.03 \mathrm{E}-04$ & $4.53 \mathrm{E}-04$ & $4.50 \mathrm{E}-04$ & $3.03 \mathrm{E}-04$ & $3.03 \mathrm{E}-04$ & $4.44 \mathrm{E}-04$ & $4.41 \mathrm{E}-04$ & $4.47 \mathrm{E}-04$ & $4.47 \mathrm{E}-04$ & $4.46 \mathrm{E}-04$ & $4.49 \mathrm{E}-04$ \\
\hline
\end{tabular}


Table 7: Projected Feed Composition After Dissolution and Dilution to 6.44 M Total Sodium Concentration no sludge; $30 \%$ of interstitial supernate remaining

\begin{tabular}{|c|c|c|c|c|c|c|c|c|c|c|c|c|c|c|c|}
\hline \multicolumn{16}{|c|}{ Concentration (M) } \\
\hline & Tank 25 & Tank 27 & Tank 28 & Tank 29 & Tank 31 & Tank 33 & Tank 34 & Tank 36 & Tank 37 & Tank 38 & Tank 41 & Tank 44 & Tank 45 & Tank 46 & Tank 47 \\
\hline $\mathrm{Na}$ & $6.44 \mathrm{E}+00$ & $6.44 \mathrm{E}+00$ & $6.44 \mathrm{E}+00$ & $6.44 \mathrm{E}+00$ & $6.44 \mathrm{E}+00$ & $6.44 \mathrm{E}+00$ & $6.44 \mathrm{E}+00$ & $6.44 \mathrm{E}+00$ & $6.44 \mathrm{E}+00$ & $6.44 \mathrm{E}+00$ & $6.44 \mathrm{E}+00$ & $6.44 \mathrm{E}+00$ & $6.44 \mathrm{E}+00$ & $6.44 \mathrm{E}+00$ & $6.44 \mathrm{E}+00$ \\
\hline $\mathrm{Al}(\mathrm{OH}) 4$ & $3.20 \mathrm{E}-01$ & 3.32E-01 & $3.20 \mathrm{E}-01$ & $4.00 \mathrm{E}-01$ & $4.01 \mathrm{E}-01$ & $3.19 \mathrm{E}-01$ & 3.17E-01 & $3.84 \mathrm{E}-01$ & $3.93 \mathrm{E}-01$ & $4.01 \mathrm{E}-01$ & $3.91 \mathrm{E}-01$ & $3.15 \mathrm{E}-01$ & $3.13 \mathrm{E}-01$ & $3.08 \mathrm{E}-01$ & $3.22 \mathrm{E}-01$ \\
\hline $\mathrm{CO} 3$ & $2.18 \mathrm{E}-01$ & $2.17 \mathrm{E}-01$ & $2.16 \mathrm{E}-01$ & $1.32 \mathrm{E}-01$ & $1.30 \mathrm{E}-01$ & $2.22 \mathrm{E}-01$ & $2.27 \mathrm{E}-01$ & $1.30 \mathrm{E}-01$ & $1.30 \mathrm{E}-01$ & $1.35 \mathrm{E}-01$ & $1.35 \mathrm{E}-01$ & $2.18 \mathrm{E}-01$ & $2.20 \mathrm{E}-01$ & $2.13 \mathrm{E}-01$ & $2.24 \mathrm{E}-01$ \\
\hline$\overline{\mathrm{C2O}}$ & $2.18 \mathrm{E}-02$ & $2.17 \mathrm{E}-02$ & $2.18 \mathrm{E}-02$ & $1.41 \mathrm{E}-02$ & $1.38 \mathrm{E}-02$ & $2.24 \mathrm{E}-02$ & $2.19 \mathrm{E}-02$ & $1.38 \mathrm{E}-02$ & $1.40 \mathrm{E}-02$ & $1.43 \mathrm{E}-02$ & $1.39 \mathrm{E}-02$ & $2.17 \mathrm{E}-02$ & $2.17 \mathrm{E}-02$ & $2.16 \mathrm{E}-02$ & $2.21 \mathrm{E}-02$ \\
\hline NO2 & $1.26 \mathrm{E}-01$ & $1.28 \mathrm{E}-01$ & $1.35 \mathrm{E}-01$ & $7.48 \mathrm{E}-01$ & $7.62 \mathrm{E}-01$ & $1.02 \mathrm{E}-01$ & $1.24 \mathrm{E}-01$ & $7.57 \mathrm{E}-01$ & $7.52 \mathrm{E}-01$ & $7.45 \mathrm{E}-01$ & $7.39 \mathrm{E}-01$ & $1.30 \mathrm{E}-01$ & $1.34 \mathrm{E}-01$ & $1.31 \mathrm{E}-01$ & $1.13 \mathrm{E}-01$ \\
\hline NO3 & $3.72 \mathrm{E}+00$ & $3.71 \mathrm{E}+00$ & $3.73 \mathrm{E}+00$ & $2.65 \mathrm{E}+00$ & $2.61 \mathrm{E}+00$ & $3.83 \mathrm{E}+00$ & $3.76 \mathrm{E}+00$ & $2.60 \mathrm{E}+00$ & $2.61 \mathrm{E}+00$ & $2.67 \mathrm{E}+00$ & $2.65 \mathrm{E}+00$ & $3.69 \mathrm{E}+00$ & $3.70 \mathrm{E}+00$ & $3.69 \mathrm{E}+00$ & $3.78 \mathrm{E}+00$ \\
\hline $\mathrm{OH}$ & $1.07 \mathrm{E}+00$ & $1.07 \mathrm{E}+00$ & $1.02 \mathrm{E}+00$ & $1.86 \mathrm{E}+00$ & $1.88 \mathrm{E}+00$ & $9.25 \mathrm{E}-01$ & $1.01 \mathrm{E}+00$ & $1.98 \mathrm{E}+00$ & $1.91 \mathrm{E}+00$ & $1.87 \mathrm{E}+00$ & $1.87 \mathrm{E}+00$ & $1.12 \mathrm{E}+00$ & $1.15 \mathrm{E}+00$ & $9.99 \mathrm{E}-01$ & $9.63 \mathrm{E}-01$ \\
\hline $\mathrm{PO4}$ & $1.64 \mathrm{E}-03$ & $1.63 \mathrm{E}-03$ & 1.77E-03 & $1.56 \mathrm{E}-02$ & $1.55 \mathrm{E}-02$ & $1.41 \mathrm{E}-03$ & & 1.57E-02 & $1.54 \mathrm{E}-02$ & $1.58 \mathrm{E}-02$ & $1.58 \mathrm{E}-02$ & $1.50 \mathrm{E}-03$ & $1.53 \mathrm{E}-03$ & $1.61 \mathrm{E}-03$ & $1.40 \mathrm{E}-03$ \\
\hline$\overline{\mathrm{SO4}}$ & $3.17 \mathrm{E}-01$ & $3.16 \mathrm{E}-01$ & $3.16 \mathrm{E}-01$ & $1.82 \mathrm{E}-01$ & $1.78 \mathrm{E}-01$ & $3.27 \mathrm{E}-01$ & $3.18 \mathrm{E}-01$ & $1.78 \mathrm{E}-01$ & $1.78 \mathrm{E}-01$ & $1.84 \mathrm{E}-01$ & $1.80 \mathrm{E}-01$ & $3.14 \mathrm{E}-01$ & $3.15 \mathrm{E}-01$ & $3.14 \mathrm{E}-01$ & $3.21 \mathrm{E}-01$ \\
\hline$\overline{\mathrm{Ag}}$ & $1.79 \mathrm{E}-08$ & $1.05 \mathrm{E}-07$ & $7.74 \mathrm{E}-08$ & $3.95 \mathrm{E}-08$ & $3.34 \mathrm{E}-08$ & $3.73 \mathrm{E}-08$ & $3.63 \mathrm{E}-08$ & $3.34 \mathrm{E}-08$ & $3.34 \mathrm{E}-08$ & $4.38 \mathrm{E}-08$ & $3.37 \mathrm{E}-08$ & $3.59 \mathrm{E}-08$ & $3.60 \mathrm{E}-08$ & $3.58 \mathrm{E}-08$ & $3.66 \mathrm{E}-08$ \\
\hline As & $3.15 \mathrm{E}-06$ & $2.85 \mathrm{E}-06$ & $3.72 \mathrm{E}-06$ & $5.15 \mathrm{E}-06$ & $5.26 \mathrm{E}-05$ & $2.57 \mathrm{E}-05$ & $6.47 \mathrm{E}-05$ & $9.14 \mathrm{E}-05$ & $6.58 \mathrm{E}-05$ & $3.56 \mathrm{E}-06$ & $4.36 \mathrm{E}-05$ & $1.09 \mathrm{E}-04$ & $1.20 \mathrm{E}-04$ & $6.56 \mathrm{E}-05$ & $4.58 \mathrm{E}-05$ \\
\hline$\overline{\mathrm{Ba}}$ & $3.12 \mathrm{E}-09$ & $3.11 \mathrm{E}-08$ & $2.34 \mathrm{E}-08$ & $2.07 \mathrm{E}-07$ & ---- & 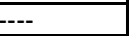 & ---- & - & ---- & 7.47E-09 & --- & 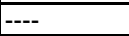 & 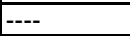 & 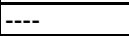 & - \\
\hline $\mathrm{Ca}$ & $4.92 \mathrm{E}-05$ & $4.90 \mathrm{E}-05$ & $4.91 \mathrm{E}-05$ & $3.19 \mathrm{E}-05$ & $3.01 \mathrm{E}-05$ & $5.22 \mathrm{E}-05$ & $4.93 \mathrm{E}-05$ & $3.01 \mathrm{E}-05$ & $3.01 \mathrm{E}-05$ & E-05 & $\mathrm{E}-01$ & $4.88 \mathrm{E}-05$ & $9 \mathrm{E}-05$ & $4.87 \mathrm{E}-05$ & $5.00 \mathrm{E}-05$ \\
\hline$\overline{C d}$ & $8.59 \mathrm{E}-08$ & $8.18 \mathrm{E}-08$ & $1.09 \mathrm{E}-07$ & $1.35 \mathrm{E}-07$ & $1.42 \mathrm{E}-06$ & $3.08 \mathrm{E}-07$ & $1.83 \mathrm{E}-06$ & $2.92 \mathrm{E}-06$ & $1.93 \mathrm{E}-06$ & $7.30 \mathrm{E}-08$ & $1.06 \mathrm{E}-06$ & $3.55 \mathrm{E}-06$ & $3.95 \mathrm{E}-06$ & $1.88 \mathrm{E}-06$ & $1.10 \mathrm{E}-06$ \\
\hline$\overline{\mathrm{Cl}}$ & $4.19 \mathrm{E}-02$ & $4.18 \mathrm{E}-02$ & $4.18 \mathrm{E}-02$ & $3.83 \mathrm{E}-02$ & 3.74E-02 & $4.28 \mathrm{E}-02$ & & 3.76E-02 & $3.75 \mathrm{E}-02$ & $3.86 \mathrm{E}-02$ & $3.78 \mathrm{E}-02$ & $4.17 \mathrm{E}-02$ & $4.19 \mathrm{E}-02$ & $4.15 \mathrm{E}-02$ & $4.21 \mathrm{E}-02$ \\
\hline Co & $4.57 \mathrm{E}-07$ & $4.66 \mathrm{E}-07$ & $3.84 \mathrm{E}-07$ & $2.05 \mathrm{E}-07$ & -07 & $1.98 \mathrm{E}-07$ & & $4.60 \mathrm{E}-07$ & E-07 & $1.99 \mathrm{E}-07$ & E-07 & $9 \mathrm{E}-07$ & $4 \mathrm{E}-07$ & E-07 & $2.79 \mathrm{E}-07$ \\
\hline $\mathrm{Cr}$ & $1.90 \mathrm{E}-05$ & $1.15 \mathrm{E}-05$ & $3.83 \mathrm{E}-05$ & $1.59 \mathrm{E}-04$ & $2.10 \mathrm{E}-04$ & $2.61 \mathrm{E}-04$ & 2.2 & $1.79 \mathrm{E}-04$ & $2.00 \mathrm{E}-04$ & $2.01 \mathrm{E}-04$ & $2.20 \mathrm{E}-04$ & $1.83 \mathrm{E}-04$ & $1.76 \mathrm{E}-04$ & $2.18 \mathrm{E}-04$ & $2.40 \mathrm{E}-04$ \\
\hline $\mathrm{Cu}$ & $1.64 \mathrm{E}-04$ & $1.69 \mathrm{E}-04$ & $1.24 \mathrm{E}-04$ & $2.99 \mathrm{E}-05$ & $8.41 \mathrm{E}-05$ & $1.84 \mathrm{E}-05$ & -04 & $1.73 \mathrm{E}-04$ & $4 \mathrm{E}-04$ & $2.56 \mathrm{E}-05$ & $11 \mathrm{E}-05$ & $2.10 \mathrm{E}-04$ & $2.34 \mathrm{E}-04$ & $1.11 \mathrm{E}-04$ & $6.50 \mathrm{E}-05$ \\
\hline $\mathrm{Fe}$ & $1.38 \mathrm{E}-06$ & $1.38 \mathrm{E}-06$ & $1.07 \mathrm{E}-06$ & $8.36 \mathrm{E}-07$ & $3.83 \mathrm{E}-06$ & $9.41 \mathrm{E}-07$ & $4.92 \mathrm{E}-06$ & 7.74E-06 & $5.15 \mathrm{E}-06$ & $1.95 \mathrm{E}-06$ & $2.90 \mathrm{E}-06$ & $9.40 \mathrm{E}-06$ & $1.05 \mathrm{E}-05$ & $5.04 \mathrm{E}-06$ & $3.00 \mathrm{E}-06$ \\
\hline $\mathbf{F}$ & $6.95 \mathrm{E}-02$ & $6.93 \mathrm{E}-02$ & $6.94 \mathrm{E}-02$ & $3.59 \mathrm{E}-02$ & $E-02$ & $7.15 \mathrm{E}-02$ & & $3.52 \mathrm{E}-02$ & $E-02$ & $\mathrm{E}-02$ & $E-02$ & $\mathrm{E}-02$ & $E-02$ & $6.87 \mathrm{E}-02$ & $7.03 \mathrm{E}-02$ \\
\hline $\mathrm{Hg}$ & $1.07 \mathrm{E}-06$ & $1.07 \mathrm{E}-06$ & $1.07 \mathrm{E}-06$ & $2.02 \mathrm{E}-06$ & $1.21 \mathrm{E}-05$ & $1.29 \mathrm{E}-05$ & -05 & $1.29 \mathrm{E}-05$ & E-05 & $1.02 \mathrm{E}-06$ & E-05 & $1.41 \mathrm{E}-05$ & $1.44 \mathrm{E}-05$ & $1.32 \mathrm{E}-05$ & $1.31 \mathrm{E}-05$ \\
\hline $\mathbf{K}$ & $7.45 \mathrm{E}-03$ & $7.64 \mathrm{E}-03$ & $7.44 \mathrm{E}-03$ & $5.24 \mathrm{E}-03$ & $5.81 \mathrm{E}-03$ & $5.09 \mathrm{E}-03$ & $E-03$ & $7.67 \mathrm{E}-03$ & $6.03 \mathrm{E}-03$ & $4.73 \mathrm{E}-03$ & $5.08 \mathrm{E}-03$ & $8.04 \mathrm{E}-03$ & $8.48 \mathrm{E}-03$ & $6.44 \mathrm{E}-03$ & $5.89 \mathrm{E}-03$ \\
\hline$\overline{\mathrm{Mg}}$ & --- & ---- & $1.01 \mathrm{E}-08$ & $1.31 \mathrm{E}-07$ & $5.17 \mathrm{E}-08$ & $1.62 \mathrm{E}-07$ & $3.24 \mathrm{E}-08$ & 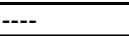 & $1.02 \mathrm{E}-08$ & $1.38 \mathrm{E}-07$ & $8.20 \mathrm{E}-08$ & 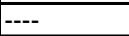 & ---- & $2.62 \mathrm{E}-08$ & $9.39 \mathrm{E}-08$ \\
\hline$\overline{M n}$ & $5.86 \mathrm{E}-08$ & $7.00 \mathrm{E}-08$ & $9.36 \mathrm{E}-08$ & $1.11 \mathrm{E}-07$ & $1.98 \mathrm{E}-07$ & $1.04 \mathrm{E}-07$ & $2.42 \mathrm{E}-07$ & 3.35E-07 & $2.44 \mathrm{E}-07$ & $5.98 \mathrm{E}-08$ & $1.66 \mathrm{E}-07$ & $3.98 \mathrm{E}-07$ & $4.35 \mathrm{E}-07$ & $2.45 \mathrm{E}-07$ & $1.75 \mathrm{E}-07$ \\
\hline Nd & ---- & --- & $\begin{array}{ll}--- \\
\end{array}$ & $4.34 \mathrm{E}-06$ & ---- & $5.85 \mathrm{E}-06$ & --- & --- & ---- & $4.75 \mathrm{E}-06$ & $1.59 \mathrm{E}-06$ & --- & ---- & --- & $2.00 \mathrm{E}-06$ \\
\hline$\overline{\mathrm{Ni}}$ & $1.10 \mathrm{E}-06$ & $1.12 \mathrm{E}-06$ & $\begin{array}{c}9.01 \mathrm{E}-07 \\
\end{array}$ & $4.30 \mathrm{E}-07$ & $6.85 \mathrm{E}-07$ & $4.00 \mathrm{E}-07$ & $8.28 \mathrm{E}-07$ & $1.11 \mathrm{E}-06$ & $8.30 \mathrm{E}-07$ & $4.13 \mathrm{E}-07$ & $5.87 \mathrm{E}-07$ & $1.32 \mathrm{E}-06$ & $1.43 \mathrm{E}-06$ & $8.37 \mathrm{E}-07$ & $6.20 \mathrm{E}-07$ \\
\hline$\overline{\mathrm{Pb}}$ & $3.00 \mathrm{E}-06$ & $2.48 \mathrm{E}-06$ & $3.41 \mathrm{E}-06$ & $5.00 \mathrm{E}-06$ & ---- & -- & --- & --- & --- & $2.97 \mathrm{E}-06$ & -- & ---- & $\begin{array}{ll}--- \\
\end{array}$ & $\begin{array}{ll}--- \\
\end{array}$ & --- \\
\hline$\overline{\mathbf{R u}}$ & $1.73 \mathrm{E}-05$ & $1.78 \mathrm{E}-05$ & $1.34 \mathrm{E}-05$ & $4.15 \mathrm{E}-06$ & $9.41 \mathrm{E}-06$ & $3.14 \mathrm{E}-06$ & $1.19 \mathrm{E}-05$ & $1.81 \mathrm{E}-05$ & $1.23 \mathrm{E}-05$ & $3.76 \mathrm{E}-06$ & $7.38 \mathrm{E}-06$ & $2.18 \mathrm{E}-05$ & $2.42 \mathrm{E}-05$ & $1.22 \mathrm{E}-05$ & $7.67 \mathrm{E}-06$ \\
\hline$\overline{\mathrm{Se}}$ & $2.23 \mathrm{E}-05$ & $1.71 \mathrm{E}-05$ & $2.87 \mathrm{E}-05$ & $6.04 \mathrm{E}-05$ & $1.62 \mathrm{E}-04$ & $2.18 \mathrm{E}-04$ & $1.67 \mathrm{E}-04$ & $1.17 \mathrm{E}-04$ & $1.46 \mathrm{E}-04$ & $5.85 \mathrm{E}-05$ & $1.74 \mathrm{E}-04$ & $1.13 \mathrm{E}-04$ & $1.02 \mathrm{E}-04$ & $1.62 \mathrm{E}-04$ & $1.90 \mathrm{E}-04$ \\
\hline$\overline{\mathrm{Si}}$ & $1.06 \mathrm{E}-04$ & $9.29 \mathrm{E}-05$ & $5.44 \mathrm{E}-04$ & $1.81 \mathrm{E}-05$ & ---- & --- &.--- & --- & ---- & $1.42 \mathrm{E}-04$ & -.- & --- & --- & --- & --- \\
\hline $\mathrm{Sr}$ & ---- & --- & $\begin{array}{ll}--- \\
\end{array}$ & $2.92 \mathrm{E}-06$ & --- & $4.05 \mathrm{E}-06$ & --- & - & --- & $3.23 \mathrm{E}-06$ & $7.95 \mathrm{E}-07$ & 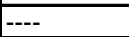 & --- & - & $1.08 \mathrm{E}-06$ \\
\hline$Z n$ & 1.99E-04 & $2.05 \mathrm{E}-04$ & \begin{tabular}{|c|}
$1.56 \mathrm{E}-04$ \\
\end{tabular} & $5.24 \mathrm{E}-05$ & $1.11 \mathrm{E}-04$ & $4.16 \mathrm{E}-05$ & $\begin{array}{c}1.39 \mathrm{E}-04 \\
\end{array}$ & 2.07E-04 & $1.43 \mathrm{E}-04$ & $4.80 \mathrm{E}-05$ & $8.82 \mathrm{E}-05$ & \begin{tabular}{|c|}
$2.49 \mathrm{E}-04$ \\
\end{tabular} & $2.76 \mathrm{E}-04$ & $1.42 \mathrm{E}-04$ & $9.19 \mathrm{E}-05$ \\
\hline$\overline{Z r}$ & $5.25 \mathrm{E}-06$ & $5.41 \mathrm{E}-06$ & $4.00 \mathrm{E}-06$ & $1.06 \mathrm{E}-06$ & $2.74 \mathrm{E}-06$ & $7.12 \mathrm{E}-07$ & $3.52 \mathrm{E}-06$ & $5.50 \mathrm{E}-06$ & $3.68 \mathrm{E}-06$ & $9.28 \mathrm{E}-07$ & $2.09 \mathrm{E}-06$ & $6.68 \mathrm{E}-06$ & $7.43 \mathrm{E}-06$ & $3.60 \mathrm{E}-06$ & $2.16 \mathrm{E}-06$ \\
\hline Insol.Sol.mg/L & 0 & 0 & 0 & 0 & 0 & 0 & 0 & 0 & 0 & 0 & 0 & & 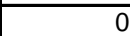 & & \\
\hline
\end{tabular}


Table 7: Projected Feed Composition After Dissolution and Dilution to 6.44 M Total Sodium Concentration no sludge; $30 \%$ of interstitial supernate (continued)

\begin{tabular}{|c|c|c|c|c|c|c|c|c|c|c|c|c|c|c|c|}
\hline \multicolumn{16}{|c|}{ Radionuclide Composition (Ci/gal) } \\
\hline & Tank 25 & Tank 27 & Tank 28 & Tank 29 & Tank 31 & Tank 33 & \begin{tabular}{|l|} 
Tank 34 \\
\end{tabular} & Tank 36 & Tank 37 & Tank 38 & Tank 41 & Tank 44 & Tank 45 & Tank 46 & Tank 47 \\
\hline C-14 & --- & $-\cdots$ & $-\cdots$ & --- & --- & --- & $-\cdots$ & $-\cdots$ & $\mid$ & - & ---- & $-\cdots$ & 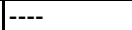 & ---- & \\
\hline $\mathrm{Ni}-59$ & ---- & ---- & --.- & $-\ldots$ & ---- & $\ldots$ & ---- & $-\overline{-}_{-}$ & $-\ldots$ & --.-- & ---- & $-\overline{-}^{-}$ & --- & ---- & ---- \\
\hline Ni-63 & --- & --- & --- & --- & $---{ }^{---}$ & --- & --- & $\ldots$ & $-{ }_{---}$ & ב--- & - & 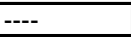 & - & $\ldots$ & ב--- \\
\hline Co-60 & $-\ldots-$ & ---- & ---- & $-\ldots$ & ---- & $-\ldots-$ & ---- & $-{ }_{--}$ & ---- & $-\cdots$ & --- & $-\cdots$ & --- & - & --- \\
\hline Se-79 & ---- & --- & ---- & --- & ---- & ---- & --- & $-\cdots$ & $-\cdots$ & $-\cdots$ & $-\cdots$ & $-\cdots$ & - & 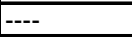 & ---- \\
\hline Tc-99 & - & $-\cdots$ & $-\cdots$ & $-\cdots$ & - & $-\cdots$ & $-\overline{-}$ & $-\cdots$ & $-\cdots$ & - & $-\overline{-}$ & $-\overline{-}$ & - & - & $-\overline{-}$ \\
\hline Ru-106 & --- & $-\cdots$ & ---- & $-\cdots$ & - & - & --- & $-\cdots$ & - & - & - & --- & - & - & ---- \\
\hline Rh-106 & $\cdots$ & ---- & $-\cdots$ & --.- & $\mid---$ & $\mid---$ & $-\cdots-$ & --.- & ---- & ---- & --- & $-\cdots$ & - & $-\cdots$ & ---- \\
\hline Sb-125 & - & - & $-\cdots$ & $-\cdots$ & - & \begin{tabular}{|c|}
$-\cdots-$ \\
\end{tabular} & $-\cdots-$ & $-\cdots$ & $\mid-\ldots$ & $\mid-\cdots$ & -..- & ---- & --- & --.- & $-\overline{--}$ \\
\hline Sn-126 & --- & --- & ---- & $-\cdots$ & ---- & \begin{tabular}{|c|}
$-\cdots-$ \\
\end{tabular} & $-\cdots$ & $-\cdots$ & ---- & $-\cdots$ & $-\cdots$ & --- & --.- & $-\overline{-}$ & $-\cdots$ \\
\hline I-129 & $-\cdots$ & \begin{tabular}{|l|}
--- \\
\end{tabular} & $-\cdots-$ & $\cdots$ & 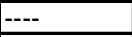 & --- & ---. & $-\ldots$ & ---- & --- & --.- & $-\cdots$ & - & - & ---- \\
\hline Cs-134 & $-\cdots-$ & --- & --- & ---- & \begin{tabular}{|c|}
--- \\
\end{tabular} & $-\cdots$ & $-\cdots$ & $-\cdots-$ & $-\cdots$ & ---- & $-\cdots$ & $-\cdots$ & --- & $-\overline{-}$ & $-\cdots$ \\
\hline Cs-135 & ---- & \begin{tabular}{|l}
--- \\
\end{tabular} & \begin{tabular}{|l}
--- \\
\end{tabular} & --- & $\begin{array}{ll}--- \\
\end{array}$ & $\begin{array}{ll}--- \\
\end{array}$ & \begin{tabular}{|l|}
---- \\
\end{tabular} & --- & $\begin{array}{ll}--- \\
\end{array}$ & $\begin{array}{ll}--- \\
\end{array}$ & --- & --- & $-\cdots$ & ---- & $\begin{array}{l}--- \\
-1\end{array}$ \\
\hline Cs-137 & $9.21 \mathrm{E}-02$ & $8.71 \mathrm{E}-02$ & \begin{tabular}{|c|}
$9.58 \mathrm{E}-02$ \\
\end{tabular} & $8.31 \mathrm{E}-02$ & \begin{tabular}{|l}
$2.25 \mathrm{E}-01$ \\
\end{tabular} & \begin{tabular}{|l}
$2.20 \mathrm{E}-03$ \\
\end{tabular} & $7.49 \mathrm{E}-02$ & $4.14 \mathrm{E}-01$ & \begin{tabular}{|l|l}
$3.01 E-01$ \\
\end{tabular} & $5.55 \mathrm{E}-03$ & $5.04 \mathrm{E}-02$ & $1.13 \mathrm{E}-01$ & $1.07 \mathrm{E}-01$ & $1.37 \mathrm{E}-01$ & $5.79 \mathrm{E}-02$ \\
\hline Ba-137m & $8.71 \mathrm{E}-02$ & $8.24 \mathrm{E}-02$ & \begin{tabular}{|c|}
$9.06 \mathrm{E}-02$ \\
\end{tabular} & $7.86 \mathrm{E}-02$ & \begin{tabular}{|l}
$2.13 \mathrm{E}-01$ \\
\end{tabular} & \begin{tabular}{|l}
$2.08 \mathrm{E}-03$ \\
\end{tabular} & $7.09 \mathrm{E}-02$ & $3.92 \mathrm{E}-01$ & \begin{tabular}{|c|c|}
$2.84 \mathrm{E}-01$ \\
\end{tabular} & $5.25 \mathrm{E}-03$ & $4.77 \mathrm{E}-02$ & 1.07E-01 & $1.01 \mathrm{E}-01$ & $1.30 \mathrm{E}-01$ & $5.48 \mathrm{E}-02$ \\
\hline Sr-90 & $6.22 \mathrm{E}-05$ & $6.20 \mathrm{E}-05$ & \begin{tabular}{|c|}
$6.21 \mathrm{E}-05$ \\
\end{tabular} & $2.23 \mathrm{E}-04$ & \begin{tabular}{|l}
$2.18 \mathrm{E}-04$ \\
\end{tabular} & \begin{tabular}{|c|}
$2.43 \mathrm{E}-04$ \\
\end{tabular} & $2.37 \mathrm{E}-04$ & $2.18 \mathrm{E}-04$ & $2.18 \mathrm{E}-04$ & $5.95 \mathrm{E}-05$ & $5.79 \mathrm{E}-05$ & $6.18 \mathrm{E}-00$ & $6.19 \mathrm{E}-05$ & $6.16 \mathrm{E}-05$ & $6.29 \mathrm{E}-\mathrm{C}$ \\
\hline Y-90 & $6.22 \mathrm{E}-05$ & $6.20 \mathrm{E}-05$ & $6.21 \mathrm{E}-05$ & $2.23 \mathrm{E}-04$ & \begin{tabular}{|l|l|}
$2.18 \mathrm{E}-04$ \\
\end{tabular} & $2.43 \mathrm{E}-04$ & \begin{tabular}{|l|l|}
$2.37 \mathrm{E}-04$ \\
\end{tabular} & $2.18 \mathrm{E}-04$ & $2.18 \mathrm{E}-04$ & $5.95 \mathrm{E}-05$ & $5.79 \mathrm{E}-05$ & $6.18 \mathrm{E}-05$ & $6.19 \mathrm{E}-05$ & $6.16 \mathrm{E}-05$ & $6.29 \mathrm{E}$ \\
\hline H-3 & ---- & ---- & ---- & ---- & \begin{tabular}{|l|}
---- \\
\end{tabular} & \begin{tabular}{|l|}
---- \\
\end{tabular} & $-\cdots$ & --- & --- & $\cdots$ & $-\ldots$ & --- & $-\ldots$ & --- & --- \\
\hline Ce-144 & ---- & $-\ldots-$ & ---- & ---- & $-\ldots-$ & $-\ldots-$ & $-\cdots-$ & $-\ldots-$ & $-\ldots-$ & ---- & ---- & --- & $-\ldots-$ & --- & - \\
\hline $\mathrm{Pr}-144$ & $-\cdots$ & \begin{tabular}{|l}
--- \\
\end{tabular} & \begin{tabular}{|l|}
--- \\
\end{tabular} & $-\cdots$ & \begin{tabular}{|l|}
--- \\
\end{tabular} & \begin{tabular}{|l|}
--- \\
\end{tabular} & \begin{tabular}{|l|}
$-\cdots-$ \\
\end{tabular} & $-\cdots$ & \begin{tabular}{|l|}
---- \\
\end{tabular} & $-\cdots-$ & --- & $-\cdots$ & --- & $-\cdots-$ & ---- \\
\hline Pm-147 & $-\cdots$ & \begin{tabular}{|l|}
$-\cdots$ \\
\end{tabular} & - & $-\cdots$ & ---- & ---- & $-\cdots$ & $-\cdots-$ & ---- & ---- & $-\cdots$ & --- & --- & $-\overline{-}$ & --- \\
\hline Eu-154 & - & - & - & ---- & $\begin{array}{ll}--- \\
\end{array}$ & - & \begin{tabular}{|l|}
$-\cdots$ \\
\end{tabular} & ---- & $\begin{array}{ll}--- \\
\end{array}$ & - & $-\cdots$ & $-\cdots$ & -- & $-\overline{-}$ & ---- \\
\hline Th-232 & ---- & $-{ }_{---}$ & $-{ }_{-1--}$ & $1.09 \mathrm{E}-11$ & \begin{tabular}{|l|}
$1.07 E-11$ \\
\end{tabular} & \begin{tabular}{|l|}
---- \\
\end{tabular} & $\mid----$ & $1.07 \mathrm{E}-11$ & \begin{tabular}{|l|}
$1.07 E-11$ \\
\end{tabular} & $-{ }_{-1}$ & - & --- & ---- & --- & $-\overline{---}$ \\
\hline $\mathrm{U}-232$ & $7.47 \mathrm{E}-11$ & \begin{tabular}{|l|l}
$7.44 \mathrm{E}-11$ \\
\end{tabular} & $7.46 \mathrm{E}-11$ & $3.11 \mathrm{E}-12$ & $3.04 \mathrm{E}-12$ & \begin{tabular}{|c|}
$1.02 \mathrm{E}-09$ \\
\end{tabular} & \begin{tabular}{|l|l}
$1.00 \mathrm{E}-0$ \\
\end{tabular} & $3.04 \mathrm{E}-12$ & \begin{tabular}{|c|}
$3.04 \mathrm{E}-12$ \\
\end{tabular} & $-\cdots$ & $-\cdots$ & $7.42 \mathrm{E}-11$ & \begin{tabular}{|l|l|}
$7.44 \mathrm{E}-11$ \\
\end{tabular} & $7.39 \mathrm{E}-11$ & $7.56 \mathrm{E}-11$ \\
\hline U-233 & 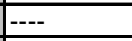 & \begin{tabular}{|l}
--- \\
-1
\end{tabular} & \begin{tabular}{|l|}
--- \\
\end{tabular} & $2.24 \mathrm{E}-09$ & $2.19 \mathrm{E}-09$ & $\begin{array}{ll}--- \\
-1\end{array}$ & ---- & $2.19 \mathrm{E}-09$ & \begin{tabular}{|c|c|}
$2.19 \mathrm{E}-09$ \\
\end{tabular} & 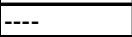 & ---- & --- & $\mid---$ & 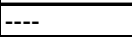 & --- \\
\hline $\mathrm{U}-234$ & & $-\cdots$ & \begin{tabular}{|l|}
--- \\
\end{tabular} & $3.16 \mathrm{E}-10$ & $3.09 \mathrm{E}-10$ & \begin{tabular}{|l|}
--- \\
\end{tabular} & \begin{tabular}{|l|}
$-\cdots$ \\
\end{tabular} & $3.09 \mathrm{E}-10$ & $3.09 \mathrm{E}-10$ & $5.22 \mathrm{E}-09$ & $5.09 \mathrm{E}-09$ & & & & \\
\hline $\mathrm{U}-235$ & $1.67 \mathrm{E}-10$ & \begin{tabular}{|l|}
$1.67 \mathrm{E}-10$ \\
\end{tabular} & \begin{tabular}{|c|}
$1.67 E-10$ \\
\end{tabular} & 8.65 & $8.44 \mathrm{E}-12$ & \begin{tabular}{|l|l|}
$1.20 \mathrm{E}-09$ \\
\end{tabular} & \begin{tabular}{|c|}
$9.40 \mathrm{E}-10$ \\
\end{tabular} & $8.44 \mathrm{E}-12$ & $8.44 \mathrm{E}-12$ & $5.27 \mathrm{E}-11$ & $5.13 \mathrm{E}-11$ & $1.66 \mathrm{E}-10$ & $1.66 \mathrm{E}-10$ & $1.65 \mathrm{E}-10$ & $1.69 \mathrm{E}-10$ \\
\hline $\mathrm{U}-236$ & & & & 3.26 & $3.18 \mathrm{E}-11$ & & & $3.18 \mathrm{E}-11$ & $3.18 \mathrm{E}-11$ & $7.96 \mathrm{E}-10$ & $7.75 \mathrm{E}-10$ & & & & \\
\hline $\mathrm{U}-238$ & $1.53 \mathrm{E}-08$ & \begin{tabular}{|c|}
$1.52 E-08$ \\
\end{tabular} & \begin{tabular}{|c|}
$1.52 E-08$ \\
\end{tabular} & $1.01 \mathrm{E}-10$ & $9.87 \mathrm{E}-11$ & \begin{tabular}{|l|}
$1.10 \mathrm{E}-07$ \\
\end{tabular} & $\begin{array}{l}6.64 \mathrm{E}-08 \\
\end{array}$ & 9.8 & 9.8 & $7.40 \mathrm{E}-12$ & $7.20 \mathrm{E}-12$ & $1.52 \mathrm{E}-08$ & $1.52 \mathrm{E}-08$ & \begin{tabular}{|c|}
$1.51 \mathrm{E}-08$ \\
\end{tabular} & $1.54 \mathrm{E}-0$ \\
\hline Np-237 & & \begin{tabular}{|l|}
--- \\
\end{tabular} & \begin{tabular}{|l|}
$-\cdots$ \\
-
\end{tabular} & & 7.94 & $9.06 \mathrm{E}-08$ & $5.13 \mathrm{E}-08$ & 7.94 & $7.94 \mathrm{E}-10$ & $4.55 \mathrm{E}-09$ & $4.43 \mathrm{E}-09$ & & & --- & \\
\hline Pu-238 & $1.27 \mathrm{E}-04$ & \begin{tabular}{|c|}
$1.26 \mathrm{E}-04$ \\
\end{tabular} & \begin{tabular}{|l|}
$1.26 \mathrm{E}-04$ \\
\end{tabular} & & \begin{tabular}{|l|}
$3.03 E-06$ \\
\end{tabular} & & & $3.03 \mathrm{E}-06$ & 3.03E-06 & $1.44 \mathrm{E}-04$ & \begin{tabular}{|l}
$1.40 \mathrm{E}-04$ \\
\end{tabular} & $1.26 \mathrm{E}-04$ & $1.26 \mathrm{E}-04$ & \begin{tabular}{|c|}
$1.25 \mathrm{E}-04$ \\
\end{tabular} & $1.28 \mathrm{E}-04$ \\
\hline Pu-239 & $1.81 \mathrm{E}-05$ & $1.80 \mathrm{E}-05$ & \begin{tabular}{|c|}
$1.80 \mathrm{E}-05$ \\
\end{tabular} & $8.05 \mathrm{E}-08$ & \begin{tabular}{|l|l|}
$7.85 \mathrm{E}-08$ \\
\end{tabular} & \begin{tabular}{|c|}
$1.32 \mathrm{E}-05$ \\
\end{tabular} & $\begin{array}{l}1.07 \mathrm{E}-05 \\
\end{array}$ & $7.85 \mathrm{E}-08$ & $7.85 \mathrm{E}-08$ & $4.43 \mathrm{E}-07$ & $4.31 \mathrm{E}-07$ & $1.79 \mathrm{E}-05$ & $1.80 \mathrm{E}-05$ & $1.79 \mathrm{E}-05$ & $1.83 \mathrm{E}-05$ \\
\hline Pu-240 & $4.04 \mathrm{E}-06$ & \begin{tabular}{|l|}
$4.03 \mathrm{E}-06$ \\
\end{tabular} & \begin{tabular}{|l|}
$4.03 E-06$ \\
\end{tabular} & $3.33 \mathrm{E}-08$ & \begin{tabular}{|c|}
$3.25 \mathrm{E}-08$ \\
\end{tabular} & $2.94 \mathrm{E}-06$ & $2.40 \mathrm{E}-06$ & $3.25 \mathrm{E}-08$ & $3.25 \mathrm{E}-08$ & $2.93 \mathrm{E}-07$ & 2.85 & $4.01 \mathrm{E}-06$ & $4.02 \mathrm{E}-06$ & $4.00 \mathrm{E}-06$ & $4.09 \mathrm{E}-1$ \\
\hline Pu-241 & $1.08 \mathrm{E}-04$ & $1.08 \mathrm{E}-04$ & $1.08 \mathrm{E}-04$ & $7.22 \mathrm{E}-07$ & $7.05 \mathrm{E}-07$ & $7.65 \mathrm{E}-05$ & $6.10 \mathrm{E}-05$ & $7.05 \mathrm{E}-07$ & $7.05 \mathrm{E}-07$ & $2.45 \mathrm{E}-05$ & $2.3 \varepsilon$ & $1.08 \mathrm{E}-04$ & $1.08 \mathrm{E}-04$ & 1.07E-04 & $1.10 \mathrm{E}-04$ \\
\hline Pu-242 & $8.31 \mathrm{E}-10$ & $8.28 \mathrm{E}-10$ & \begin{tabular}{|c|}
$8.30 \mathrm{E}-10$ \\
\end{tabular} & $2.21 \mathrm{E}-11$ & $2.16 \mathrm{E}-11$ & $6.05 \mathrm{E}-10$ & & $2.16 \mathrm{E}-11$ & $2.16 \mathrm{E}-11$ & $5.49 \mathrm{E}-09$ & $5.35 \mathrm{E}-09$ & $8.25 \mathrm{E}-10$ & $8.28 \mathrm{E}-10$ & $8.23 \mathrm{E}-10$ & $8.41 \mathrm{E}-10$ \\
\hline Am-241 & ---- & \begin{tabular}{|l|l|}
--- \\
\end{tabular} & \begin{tabular}{|l|}
--- \\
\end{tabular} & 9.14E-07 & \begin{tabular}{|c|}
$8.92 E-07$ \\
\end{tabular} & $1.38 \mathrm{E}-04$ & $1.37 \mathrm{E}-04$ & $8.92 \mathrm{E}-07$ & $8.92 \mathrm{E}-07$ & $7.66 \mathrm{E}-07$ & $7.46 \mathrm{E}-07$ & -- & --- & --- & $\cdots$ \\
\hline Am-24 & $\overline{---}$ & --- & --- & $1.12 \mathrm{E}-09$ & \begin{tabular}{|c|}
$1.09 \mathrm{E}-09$ \\
\end{tabular} & $1.88 \mathrm{E}-07$ & 1.87E-07 & $1.09 \mathrm{E}-09$ & $1.09 \mathrm{E}-09$ & $9.55 \mathrm{E}-10$ & $9.30 \mathrm{E}-10$ & - & $-\cdots$ & - & - \\
\hline $\mathrm{Cm}-24$ & $-\ldots-$ & --- & $---\cdot$ & $-\ldots$ & & & 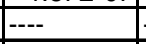 & 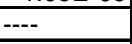 & & & 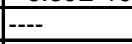 & $-\overline{-}$ & $\begin{array}{l}--- \\
\end{array}$ & --- & --- \\
\hline $\mathrm{Cm}-24$ & $1.57 \mathrm{E}-09$ & $1.56 \mathrm{E}-09$ & \begin{tabular}{|c|}
$1.57 \mathrm{E}-09$ \\
\end{tabular} & $2.68 \mathrm{E}-09$ & \begin{tabular}{|l|}
$2.61 \mathrm{E}-09$ \\
\end{tabular} & \begin{tabular}{|l|}
$7.31 \mathrm{E}-08$ \\
\end{tabular} & 7.01E-08 & $2.61 \mathrm{E}-09$ & \begin{tabular}{|l|}
$2.61 \mathrm{E}-09$ \\
\end{tabular} & \begin{tabular}{|l|}
$1.30 \mathrm{E}-08$ \\
\end{tabular} & $1.26 \mathrm{E}-08$ & $1.56 \mathrm{E}-09$ & $1.56 \mathrm{E}-09$ & $1.55 \mathrm{E}-09$ & \begin{tabular}{|c|}
$1.59 \mathrm{E}-09$ \\
\end{tabular} \\
\hline Cm-245 & $6.19 \mathrm{E}-16$ & \begin{tabular}{|l|}
$6.17 \mathrm{E}-16$ \\
\end{tabular} & \begin{tabular}{|c|}
$6.18 \mathrm{E}-16$ \\
\end{tabular} & $3.30 \mathrm{E}-13$ & \begin{tabular}{|l|}
$3.22 \mathrm{E}-13$ \\
\end{tabular} & \begin{tabular}{|l|}
$2.88 \mathrm{E}-14$ \\
\end{tabular} & \begin{tabular}{|r|}
$2.87 \mathrm{E}-14$ \\
\end{tabular} & $3.22 \mathrm{E}-13$ & $3.22 \mathrm{E}-13$ & \begin{tabular}{|l}
$7.97 \mathrm{E}-13$ \\
\end{tabular} & $7.76 \mathrm{E}-13$ & $6.14 \mathrm{E}-16$ & $6.16 \mathrm{E}-16$ & $6.12 \mathrm{E}-16$ & \begin{tabular}{|l}
$6.26 \mathrm{E}-16$ \\
\end{tabular} \\
\hline Total Alpha & $1.49 \mathrm{E}-04$ & \begin{tabular}{|c|}
$1.48 \mathrm{E}-04$ \\
\end{tabular} & \begin{tabular}{|c|}
$1.48 \mathrm{E}-04$ \\
\end{tabular} & $4.14 \mathrm{E}-06$ & \begin{tabular}{|l|}
$4.04 \mathrm{E}-06$ \\
\end{tabular} & \begin{tabular}{|l|}
$1.54 \mathrm{E}-04$ \\
\end{tabular} & \begin{tabular}{|c|}
$1.51 \mathrm{E}-04$ \\
\end{tabular} & $4.04 \mathrm{E}-06$ & $4.04 \mathrm{E}-06$ & $1.45 \mathrm{E}-04$ & $1.41 \mathrm{E}-04$ & $1.48 \mathrm{E}-04$ & $1.48 \mathrm{E}-04$ & $1.47 \mathrm{E}-04$ & $1.50 \mathrm{E}-04$ \\
\hline
\end{tabular}


Table 8: Projected Feed Composition After Dissolution and Dilution to 6.44 M Total Sodium Concentration $600 \mathrm{mg} / \mathrm{L}$ sludge; $20 \%$ of interstitial supernate remaining

\begin{tabular}{|c|c|c|c|c|c|c|c|c|c|c|c|c|c|c|c|}
\hline \multicolumn{16}{|c|}{ Concentration (M) } \\
\hline & Tank 25 & Tank 27 & Tank 28 & Tank 29 & Tank 31 & Tank 33 & Tank 34 & Tank 36 & Tank 37 & Tank 38 & Tank 41 & Tank 44 & Tank 45 & Tank 46 & Tank 47 \\
\hline $\mathrm{Na}$ & $6.44 \mathrm{E}+00$ & $6.44 \mathrm{E}+00$ & $6.44 \mathrm{E}+00$ & $6.44 \mathrm{E}+00$ & $6.44 \mathrm{E}+00$ & $6.44 \mathrm{E}+00$ & $6.44 \mathrm{E}+00$ & $6.44 \mathrm{E}+00$ & $6.44 \mathrm{E}+00$ & $6.44 \mathrm{E}+00$ & $6.44 \mathrm{E}+00$ & $6.44 \mathrm{E}+00$ & $6.44 \mathrm{E}+00$ & $6.44 \mathrm{E}+00$ & $6.44 \mathrm{E}+00$ \\
\hline $\mathrm{Al}(\mathrm{OH}) 4$ & $3.21 \mathrm{E}-01$ & $3.29 \mathrm{E}-01$ & $3.21 \mathrm{E}-01$ & $3.99 \mathrm{E}-01$ & $4.00 \mathrm{E}-01$ & $3.20 \mathrm{E}-01$ & $3.19 \mathrm{E}-01$ & $3.89 \mathrm{E}-01$ & $3.95 \mathrm{E}-01$ & $4.00 \mathrm{E}-01$ & 3.94E-01 & $3.18 \mathrm{E}-01$ & $3.16 \mathrm{E}-01$ & $3.12 \mathrm{E}-01$ & $3.22 \mathrm{E}-01$ \\
\hline $\mathrm{CO}$ & $20 \mathrm{E}-01$ & $2.19 \mathrm{E}-01$ & $2.18 \mathrm{E}-01$ & $1.33 \mathrm{E}-01$ & $1.31 \mathrm{E}-01$ & $2.23 \mathrm{E}-01$ & $2.26 \mathrm{E}-01$ & $1.31 \mathrm{E}-01$ & $1.31 \mathrm{E}-01$ & $1.35 \mathrm{E}-01$ & $1.34 \mathrm{E}-01$ & $2.20 \mathrm{E}-01$ & $2.22 \mathrm{E}-01$ & $2.17 \mathrm{E}-01$ & $2.24 \mathrm{E}-01$ \\
\hline C2O4 & $2.21 \mathrm{E}-02$ & $2.20 \mathrm{E}-02$ & $2.20 \mathrm{E}-02$ & $1.41 \mathrm{E}-02$ & $1.39 \mathrm{E}-02$ & $2.25 \mathrm{E}-02$ & $2.21 \mathrm{E}-02$ & $1.39 \mathrm{E}-02$ & $1.40 \mathrm{E}-02$ & $1.42 \mathrm{E}-02$ & $1.40 \mathrm{E}-02$ & $2.19 \mathrm{E}-02$ & $2.20 \mathrm{E}-02$ & $2.19 \mathrm{E}-02$ & $2.22 \mathrm{E}-02$ \\
\hline NO2 & $1.19 \mathrm{E}-01$ & $1.20 \mathrm{E}-01$ & $1.24 \mathrm{E}-01$ & $7.46 \mathrm{E}-01$ & $7.56 \mathrm{E}-01$ & $1.02 \mathrm{E}-01$ & $1.17 \mathrm{E}-01$ & $7.52 \mathrm{E}-01$ & $7.48 \mathrm{E}-01$ & $7.44 \mathrm{E}-01$ & $7.40 \mathrm{E}-01$ & $1.21 \mathrm{E}-01$ & $1.24 \mathrm{E}-01$ & $1.22 \mathrm{E}-01$ & $1.09 \mathrm{E}-01$ \\
\hline NO3 & $3.76 \mathrm{E}+00$ & $3.75 \mathrm{E}+00$ & $3.76 \mathrm{E}+00$ & $2.65 \mathrm{E}+00$ & $2.63 \mathrm{E}+00$ & $3.83 \mathrm{E}+00$ & $3.78 \mathrm{E}+00$ & $2.62 \mathrm{E}+00$ & $2.63 \mathrm{E}+00$ & $2.67 \mathrm{E}+00$ & $2.66 \mathrm{E}+00$ & $3.74 \mathrm{E}+00$ & $3.74 \mathrm{E}+00$ & $3.74 \mathrm{E}+00$ & $3.80 \mathrm{E}+00$ \\
\hline $\mathrm{OH}$ & $1.02 \mathrm{E}+00$ & $1.02 \mathrm{E}+00$ & $9.85 \mathrm{E}-01$ & $1.85 \mathrm{E}+00$ & $1.87 \mathrm{E}+00$ & $9.20 \mathrm{E}-01$ & $9.75 \mathrm{E}-01$ & $1.94 \mathrm{E}+00$ & $1.89 \mathrm{E}+00$ & $1.86 \mathrm{E}+00$ & $1.86 \mathrm{E}+00$ & $1.05 \mathrm{E}+00$ & $1.07 \mathrm{E}+00$ & $9.69 \mathrm{E}-01$ & $9.45 \mathrm{E}-01$ \\
\hline $\mathrm{PO4}$ & $1.53 \mathrm{E}-03$ & $1.53 \mathrm{E}-03$ & $1.62 \mathrm{E}-03$ & $1.57 \mathrm{E}-02$ & $1.56 \mathrm{E}-02$ & $1.37 \mathrm{E}-03$ & $1.37 \mathrm{E}-03$ & $1.57 \mathrm{E}-02$ & $1.55 \mathrm{E}-02$ & $1.57 \mathrm{E}-02$ & $1.57 \mathrm{E}-02$ & $1.44 \mathrm{E}-03$ & $1.45 \mathrm{E}-03$ & $1.51 \mathrm{E}-03$ & $1.37 \mathrm{E}-03$ \\
\hline SO4 & $21 \mathrm{E}-01$ & $3.20 \mathrm{E}-01$ & $3.21 \mathrm{E}-01$ & 3E-01 & $1.80 \mathrm{E}-01$ & $3.28 \mathrm{E}-01$ & E-01 & $\mathrm{E}-01$ & $1.80 \mathrm{E}-01$ & $1.85 \mathrm{E}-01$ & $1.82 \mathrm{E}-01$ & $\mathrm{E}-01$ & DE-01 & $9 \mathrm{E}-01$ & $4 \mathrm{E}-01$ \\
\hline $\mathbf{A g}$ & $1.21 \mathrm{E}-08$ & $7.10 \mathrm{E}-08$ & $5.23 \mathrm{E}-08$ & $2.65 \mathrm{E}-08$ & $2.26 \mathrm{E}-08$ & $2.49 \mathrm{E}-08$ & $2.45 \mathrm{E}-08$ & $2.26 \mathrm{E}-08$ & $2.26 \mathrm{E}-08$ & $2.92 \mathrm{E}-08$ & $2.27 \mathrm{E}-08$ & $2.43 \mathrm{E}-08$ & $2.44 \mathrm{E}-08$ & $2.43 \mathrm{E}-08$ & $2.46 \mathrm{E}-08$ \\
\hline As & $2.13 \mathrm{E}-06$ & $1.93 \mathrm{E}-06$ & $2.51 \mathrm{E}-06$ & $3.45 \mathrm{E}-06$ & $3.55 \mathrm{E}-05$ & $1.72 \mathrm{E}-05$ & $4.37 \mathrm{E}-05$ & $6.17 \mathrm{E}-05$ & $4.44 \mathrm{E}-05$ & $2.38 \mathrm{E}-06$ & $2.94 \mathrm{E}-05$ & $7.38 \mathrm{E}-05$ & $8.09 \mathrm{E}-05$ & $4.45 \mathrm{E}-05$ & $3.08 \mathrm{E}-05$ \\
\hline$\overline{B a}$ & $2.11 \mathrm{E}-09$ & $2.11 \mathrm{E}-08$ & $1.58 \mathrm{E}-08$ & E-07 & --- & --- & --- & --- & -- & $4.99 \mathrm{E}-09$ & --- & --- & --- & -- & -- \\
\hline $\mathrm{Ca}$ & $4.98 \mathrm{E}-05$ & $4.97 \mathrm{E}-05$ & $4.98 \mathrm{E}-05$ & $7 \mathrm{E}-05$ & $3.05 \mathrm{E}-05$ & $5.19 \mathrm{E}-05$ & $4.99 \mathrm{E}-05$ & $3.05 \mathrm{E}-05$ & $3.05 \mathrm{E}-05$ & $3.20 \mathrm{E}-05$ & $3.06 \mathrm{E}-01$ & E-05 & $4.97 \mathrm{E}-05$ & $4.95 \mathrm{E}-05$ & $5.04 \mathrm{E}-05$ \\
\hline Cd & $5.80 \mathrm{E}-08$ & $\mathrm{E}-08$ & E-08 & E-08 & $9.57 \mathrm{E}-07$ & $2.06 \mathrm{E}-07$ & $E-06$ & E-06 & $\mathrm{E}-06$ & $4.88 \mathrm{E}-08$ & E-07 & $\mathrm{E}-06$ & $3 \mathrm{E}-06$ & $1.27 \mathrm{E}-06$ & 7E-07 \\
\hline$\overline{\mathrm{Cl}}$ & $4.23 \mathrm{E}-02$ & $4.22 \mathrm{E}-02$ & $4.23 \mathrm{E}-02$ & $3.84 \mathrm{E}-02$ & $3.79 \mathrm{E}-02$ & $4.29 \mathrm{E}-02$ & E-02 & $3.80 \mathrm{E}-02$ & $3.79 \mathrm{E}-02$ & $3.87 \mathrm{E}-02$ & $3.81 \mathrm{E}-02$ & $4.21 \mathrm{E}-02$ & $4.23 \mathrm{E}-02$ & $4.20 \mathrm{E}-02$ & $4.24 \mathrm{E}-02$ \\
\hline Co & $3.09 \mathrm{E}-07$ & $3.15 \mathrm{E}-07$ & $2.60 \mathrm{E}-07$ & $1.37 \mathrm{E}-07$ & $2.02 \mathrm{E}-07$ & $1.32 \mathrm{E}-07$ & $2.41 \mathrm{E}-07$ & $3.11 \mathrm{E}-07$ & $2.39 \mathrm{E}-07$ & $1.33 \mathrm{E}-07$ & $1.77 \mathrm{E}-07$ & $3.65 \mathrm{E}-07$ & $3.95 \mathrm{E}-07$ & $2.44 \mathrm{E}-07$ & $1.88 \mathrm{E}-07$ \\
\hline$\overline{\mathrm{Cr}}$ & $E-05$ & E-06 & $2.59 \mathrm{E}-05$ & $E-04$ & $1.42 \mathrm{E}-04$ & $1.75 \mathrm{E}-04$ & $=-04$ & E-04 & E-04 & E-04 & $\mathrm{E}-04$ & E-04 & E-04 & $8 \mathrm{E}-04$ & $1 \mathrm{E}-04$ \\
\hline $\mathrm{Cu}$ & $E-04$ & E-04 & $8.39 \mathrm{E}-05$ & E-05 & $5.68 \mathrm{E}-05$ & $1.23 \mathrm{E}-05$ & E-05 & $7 \mathrm{E}-04$ & E-05 & $1 \mathrm{E}-05$ & $4.25 \mathrm{E}-05$ & $2 \mathrm{E}-04$ & 9E-04 & $7.54 \mathrm{E}-05$ & $8 \mathrm{E}-05$ \\
\hline $\mathrm{Fe}$ & E-07 & E-07 & $7.26 \mathrm{E}-07$ & -07 & $2.58 \mathrm{E}-06$ & $6.30 \mathrm{E}-07$ & & -06 & E-06 & E-06 & E-06 & E-06 & BE-06 & $1 \mathrm{E}-06$ & $2 \mathrm{E}-06$ \\
\hline $\bar{F}$ & $7.04 \mathrm{E}-02$ & $7.02 \mathrm{E}-02$ & $7.03 \mathrm{E}-02$ & $3.61 \mathrm{E}-02$ & $3.56 \mathrm{E}-02$ & $7.17 \mathrm{E}-02$ & $7.06 \mathrm{E}-02$ & $3.56 \mathrm{E}-02$ & $3.56 \mathrm{E}-02$ & $3.63 \mathrm{E}-02$ & $3.57 \mathrm{E}-02$ & $7.00 \mathrm{E}-02$ & $7.02 \mathrm{E}-02$ & $6.98 \mathrm{E}-02$ & $7.09 \mathrm{E}-02$ \\
\hline $\mathrm{Hg}$ & $7.23 \mathrm{E}-07$ & $7.21 \mathrm{E}-07$ & $7.22 \mathrm{E}-07$ & $1.36 \mathrm{E}-06$ & $8.20 \mathrm{E}-06$ & $8.61 \mathrm{E}-06$ & $9.01 \mathrm{E}-06$ & $8.74 \mathrm{E}-06$ & $8.38 \mathrm{E}-06$ & $6.83 \mathrm{E}-07$ & $8.12 \mathrm{E}-06$ & $9.57 \mathrm{E}-06$ & $9.73 \mathrm{E}-06$ & $8.95 \mathrm{E}-06$ & $8.79 \mathrm{E}-06$ \\
\hline $\mathrm{K}$ & $6.61 \mathrm{E}-03$ & $6.74 \mathrm{E}-03$ & $6.60 \mathrm{E}-03$ & $E-03$ & $5.38 \mathrm{E}-03$ & $5.01 \mathrm{E}-03$ & -03 & $6.64 \mathrm{E}-03$ & $3 \mathrm{E}-03$ & $\mathrm{E}-03$ & $\mathrm{E}-03$ & $7.01 \mathrm{E}-03$ & $7.31 \mathrm{E}-03$ & $5.93 \mathrm{E}-03$ & $5 \mathrm{E}-03$ \\
\hline$\overline{\mathrm{Mg}}$ & -- & $-\cdots$ & $6.80 \mathrm{E}-09$ & E-08 & $3.49 \mathrm{E}-08$ & $1.08 \mathrm{E}-07$ & $E-08$ & $-\cdots$ & E-09 & E-08 & $3 \mathrm{E}-08$ & -- & --- & $1.78 \mathrm{E}-08$ & $32 \mathrm{E}-08$ \\
\hline$\overline{M n}$ & $3.96 \mathrm{E}-08$ & $4.74 \mathrm{E}-08$ & $6.32 \mathrm{E}-08$ & 3E-08 & $1.34 \mathrm{E}-07$ & $6.99 \mathrm{E}-08$ & E-07 & $2.26 \mathrm{E}-07$ & $1.65 \mathrm{E}-07$ & $3.99 \mathrm{E}-08$ & $1.12 \mathrm{E}-07$ & $2.69 \mathrm{E}-07$ & $2.94 \mathrm{E}-07$ & $1.66 \mathrm{E}-07$ & $8 \mathrm{E}-07$ \\
\hline Nd & --- & ---- & $-\overline{-}^{-}$ & $2.91 \mathrm{E}-06$ & --- & $3.91 \mathrm{E}-06$ & -- & --- & --- & $3.17 \mathrm{E}-06$ & $1.07 \mathrm{E}-06$ & --- & --- & -- & $1.35 \mathrm{E}-06$ \\
\hline $\mathrm{Ni}$ & $7.40 \mathrm{E}-07$ & $7.57 \mathrm{E}-07$ & $6.09 \mathrm{E}-07$ & $2.88 \mathrm{E}-07$ & $4.63 \mathrm{E}-07$ & $2.68 \mathrm{E}-07$ & $5.59 \mathrm{E}-07$ & $7.52 \mathrm{E}-07$ & $5.61 \mathrm{E}-07$ & $2.76 \mathrm{E}-07$ & $3.95 \mathrm{E}-07$ & $8.91 \mathrm{E}-07$ & $9.69 \mathrm{E}-07$ & $5.67 \mathrm{E}-07$ & $4.17 \mathrm{E}-07$ \\
\hline $\mathrm{Pb}$ & $2.03 \mathrm{E}-06$ & $1.67 \mathrm{E}-06$ & $2.31 \mathrm{E}-06$ & $3.35 \mathrm{E}-06$ & -- & ---- & ---- & --- & --- & $1.98 \mathrm{E}-06$ & ---- & --- & ---- & --- & --- \\
\hline $\mathbf{R u}$ & $1.17 \mathrm{E}-05$ & $1.21 \mathrm{E}-05$ & $9.07 \mathrm{E}-06$ & E-06 & $6.36 \mathrm{E}-06$ & $2.10 \mathrm{E}-06$ & $E-06$ & $\mathrm{E}-05$ & $8.34 \mathrm{E}-06$ & $2.51 \mathrm{E}-06$ & E-06 & E-05 & E-05 & $8.24 \mathrm{E}-06$ & $5.16 \mathrm{E}-06$ \\
\hline $\mathrm{Se}$ & $1.51 \mathrm{E}-05$ & $1.15 \mathrm{E}-05$ & $1.94 \mathrm{E}-05$ & $5 \mathrm{E}-05$ & $1.09 \mathrm{E}-04$ & $1.46 \mathrm{E}-04$ & E-04 & $0 \mathrm{E}-05$ & $9.89 \mathrm{E}-05$ & $3.90 \mathrm{E}-05$ & E-04 & $7.68 \mathrm{E}-05$ & $6.90 \mathrm{E}-05$ & $1.10 \mathrm{E}-04$ & $1.28 \mathrm{E}-04$ \\
\hline $\mathbf{S i}$ & $7.17 \mathrm{E}-05$ & $6.28 \mathrm{E}-05$ & $3.68 \mathrm{E}-04$ & $1.21 \mathrm{E}-05$ & --- & --- & -- & --- & --- & $9.51 \mathrm{E}-05$ & $-\overline{-}^{-}$ & -- & -- & -- & -- \\
\hline $\mathrm{Sr}$ & -- & ---- & -- & $1.96 \mathrm{E}-06$ & --- & $2.71 \mathrm{E}-06$ & --- & --- & --- & $2.16 \mathrm{E}-06$ & $5.35 \mathrm{E}-07$ & - & - & - & $7.27 \mathrm{E}-07$ \\
\hline$Z \mathbf{z n}$ & 1.35E-04 & 1.39E-04 & $1.05 \mathrm{E}-04$ & $3.51 \mathrm{E}-05$ & $7.48 \mathrm{E}-05$ & $2.79 \mathrm{E}-05$ & 9.39E-05 & $1.40 \mathrm{E}-04$ & $9.69 \mathrm{E}-05$ & $3.20 \mathrm{E}-05$ & 5.94E-05 & $1.69 \mathrm{E}-04$ & $1.86 \mathrm{E}-04$ & $9.61 \mathrm{E}-05$ & $6.19 \mathrm{E}-05$ \\
\hline$\overline{Z r}$ & $3.55 \mathrm{E}-06$ & $3.66 \mathrm{E}-06$ & $2.70 \mathrm{E}-06$ & $7.09 \mathrm{E}-07$ & $1.85 \mathrm{E}-06$ & $4.76 \mathrm{E}-07$ & $2.37 \mathrm{E}-06$ & $3.72 \mathrm{E}-06$ & $2.48 \mathrm{E}-06$ & $6.19 \mathrm{E}-07$ & $1.41 \mathrm{E}-06$ & $4.52 \mathrm{E}-06$ & $5.03 \mathrm{E}-06$ & $2.44 \mathrm{E}-06$ & $1.46 \mathrm{E}-06$ \\
\hline Insol.Sol.m & 600 & 600 & 600 & 600 & 600 & 600 & 600 & 600 & 600 & 600 & 600 & 600 & 600 & 600 & 600 \\
\hline
\end{tabular}


Table 8: Projected Feed Composition After Dissolution and Dilution to 6.44 M Total Sodium Concentration $600 \mathrm{mg} / \mathrm{L}$ sludge; $20 \%$ of interstitial supernate remaining (continued)

\begin{tabular}{|c|c|c|c|c|c|c|c|c|c|c|c|c|c|c|c|}
\hline \multicolumn{16}{|c|}{ Radionuclide Composition (Ci/gal) } \\
\hline & Tank 25 & Tank 27 & Tank 28 & Tank 29 & Tank 31 & Tank 33 & Tank 34 & Tank 36 & Tank 37 & Tank 38 & Tank 41 & Tank 44 & Tank 45 & Tank 46 & Tank 47 \\
\hline C-14 & $3.37 \mathrm{E}-09$ & 3.37E-09 & $3.37 \mathrm{E}-09$ & 3.37E-09 & 3.37E-09 & $3.37 \mathrm{E}-09$ & 3.37E-09 & $3.37 \mathrm{E}-09$ & 3.37E-09 & $3.37 \mathrm{E}-09$ & $3.37 \mathrm{E}-09$ & 3.37E-09 & $3.37 \mathrm{E}-09$ & $3.37 \mathrm{E}-09$ & 3.37E-09 \\
\hline Ni-59 & $1.94 \mathrm{E}-06$ & $1.94 \mathrm{E}-06$ & $1.94 \mathrm{E}-06$ & $1.94 \mathrm{E}-06$ & $1.94 \mathrm{E}-06$ & $1.94 \mathrm{E}-06$ & $1.94 \mathrm{E}-06$ & $1.94 \mathrm{E}-06$ & $1.94 \mathrm{E}-06$ & $1.94 \mathrm{E}-06$ & $1.94 \mathrm{E}-06$ & $1.94 \mathrm{E}-06$ & $1.94 \mathrm{E}-06$ & $1.94 \mathrm{E}-06$ & $1.94 \mathrm{E}-06$ \\
\hline $\mathrm{Ni}-63$ & $3.01 \mathrm{E}-09$ & $3.01 \mathrm{E}-09$ & $3.01 \mathrm{E}-09$ & $3.01 \mathrm{E}-09$ & $3.01 \mathrm{E}-09$ & $3.01 \mathrm{E}-09$ & $3.01 \mathrm{E}-09$ & $3.01 \mathrm{E}-09$ & $3.01 \mathrm{E}-09$ & $3.01 \mathrm{E}-09$ & $3.01 \mathrm{E}-09$ & $3.01 \mathrm{E}-09$ & $3.01 \mathrm{E}-09$ & $3.01 \mathrm{E}-09$ & $3.01 \mathrm{E}-09$ \\
\hline Co-60 & $3.09 \mathrm{E}-04$ & $3.09 \mathrm{E}-04$ & $3.09 \mathrm{E}-04$ & $3.09 \mathrm{E}-04$ & $3.09 \mathrm{E}-04$ & & $3.09 \mathrm{E}-04$ & $3.09 \mathrm{E}-04$ & $3.09 \mathrm{E}-04$ & $3.09 \mathrm{E}-04$ & $3.09 \mathrm{E}-04$ & $3.09 \mathrm{E}-04$ & $3.09 \mathrm{E}-04$ & $3.09 \mathrm{E}-04$ & \\
\hline Se-79 & $1.14 \mathrm{E}-06$ & $1.14 \mathrm{E}-06$ & $1.14 \mathrm{E}-06$ & $1.14 \mathrm{E}-06$ & $1.14 \mathrm{E}-06$ & $1.14 \mathrm{E}-06$ & $1.14 \mathrm{E}-06$ & $1.14 \mathrm{E}-06$ & $1.14 \mathrm{E}-06$ & $1.14 \mathrm{E}-06$ & $1.14 \mathrm{E}-06$ & $1.14 \mathrm{E}-06$ & $1.14 \mathrm{E}-06$ & $1.14 \mathrm{E}-06$ & $1.14 \mathrm{E}-06$ \\
\hline TC-99 & $1.94 \mathrm{E}-05$ & $1.94 \mathrm{E}-05$ & $1.94 \mathrm{E}-05$ & $1.94 \mathrm{E}-05$ & $1.94 \mathrm{E}-05$ & $1.94 \mathrm{E}-05$ & $1.94 \mathrm{E}-05$ & $1.94 \mathrm{E}-05$ & 1.94E-05 & $1.94 \mathrm{E}-05$ & 1.94E-05 & $1.94 \mathrm{E}-05$ & $1.94 \mathrm{E}-05$ & 1.94E-05 & $1.94 \mathrm{E}-05$ \\
\hline Ru-106 & $7.18 \mathrm{E}-06$ & $7.18 \mathrm{E}-06$ & $7.18 \mathrm{E}-06$ & $7.18 \mathrm{E}-06$ & $7.18 \mathrm{E}-06$ & $7.18 \mathrm{E}-06$ & $7.18 \mathrm{E}-06$ & $7.18 \mathrm{E}-06$ & $7.18 \mathrm{E}-06$ & $7.18 \mathrm{E}-06$ & $7.18 \mathrm{E}-06$ & $7.18 \mathrm{E}-06$ & $7.18 \mathrm{E}-06$ & $7.18 \mathrm{E}-06$ & $7.18 \mathrm{E}-06$ \\
\hline Rh-106 & $7.18 \mathrm{E}-06$ & $7.18 \mathrm{E}-06$ & $7.18 \mathrm{E}-06$ & $7.18 \mathrm{E}-06$ & $7.18 \mathrm{E}-06$ & $7.18 \mathrm{E}-06$ & $7.18 \mathrm{E}-06$ & $7.18 \mathrm{E}-06$ & $7.18 \mathrm{E}-06$ & $7.18 \mathrm{E}-06$ & $7.18 \mathrm{E}-06$ & $7.18 \mathrm{E}-06$ & $7.18 \mathrm{E}-06$ & $7.18 \mathrm{E}-06$ & $7.18 \mathrm{E}-06$ \\
\hline Sb-125 & $1.84 \mathrm{E}-04$ & $1.84 \mathrm{E}-04$ & $1.84 \mathrm{E}-04$ & $1.84 \mathrm{E}-04$ & $1.84 \mathrm{E}-04$ & & $1.84 \mathrm{E}-04$ & $1.84 \mathrm{E}-04$ & $1.84 \mathrm{E}-04$ & $1.84 \mathrm{E}-04$ & $1.84 \mathrm{E}-04$ & $1.84 \mathrm{E}-04$ & $1.84 \mathrm{E}-04$ & $1.84 \mathrm{E}-04$ & $1.84 \mathrm{E}-04$ \\
\hline Sn-126 & $E-06$ & $1.53 \mathrm{E}-06$ & $1.53 \mathrm{E}-06$ & $1.53 \mathrm{E}-06$ & $1.53 \mathrm{E}-06$ & $1.53 \mathrm{E}$ & 1.53 & $1.53 \mathrm{E}-06$ & $1.53 \mathrm{E}-06$ & $1.53 \mathrm{E}-06$ & $1.53 \mathrm{E}-06$ & $1.53 \mathrm{E}-06$ & $1.53 \mathrm{E}-06$ & $1.53 \mathrm{E}-06$ & $3 \mathrm{E}-06$ \\
\hline I-129 & $1.79 \mathrm{E}-10$ & $1.79 \mathrm{E}-10$ & $1.79 \mathrm{E}-10$ & $1.79 \mathrm{E}-10$ & $1.79 \mathrm{E}-10$ & $1.79 \mathrm{E}-10$ & $1.79 \mathrm{E}-10$ & $1.79 \mathrm{E}-10$ & $1.79 \mathrm{E}-10$ & $1.79 \mathrm{E}-10$ & $1.79 \mathrm{E}-10$ & $1.79 \mathrm{E}-10$ & 9E-10 & $1.79 \mathrm{E}-10$ & $9 \mathrm{E}-10$ \\
\hline Cs-134 & $8.05 \mathrm{E}-06$ & $8.05 \mathrm{E}-06$ & $8.05 \mathrm{E}-06$ & $8.05 \mathrm{E}-06$ & $8.05 \mathrm{E}-06$ & $8.05 \mathrm{E}-06$ & $8.05 \mathrm{E}-06$ & $8.05 \mathrm{E}-06$ & $8.05 \mathrm{E}-06$ & $8.05 \mathrm{E}-06$ & $8.05 \mathrm{E}-06$ & $8.05 \mathrm{E}-06$ & $8.05 \mathrm{E}-06$ & $8.05 \mathrm{E}-06$ & $8.05 \mathrm{E}-06$ \\
\hline Cs-135 & $1.37 \mathrm{E}-08$ & $1.37 \mathrm{E}-08$ & $1.37 \mathrm{E}-08$ & $1.37 \mathrm{E}-08$ & $1.37 \mathrm{E}-08$ & $1.37 \mathrm{E}-08$ & $1.37 \mathrm{E}-08$ & $1.37 \mathrm{E}-08$ & $1.37 \mathrm{E}-08$ & $1.37 \mathrm{E}-08$ & $1.37 \mathrm{E}-08$ & $1.37 \mathrm{E}-08$ & 37E-08 & $1.37 \mathrm{E}-08$ & $37 \mathrm{E}-08$ \\
\hline Cs-137 & -02 & E-02 & $6.88 \mathrm{E}-02$ & 7E-02 & $1.56 \mathrm{E}-01$ & & 5.46 & $2.84 \mathrm{E}-01$ & $7 \mathrm{E}-01$ & $7.78 \mathrm{E}-03$ & $3.80 \mathrm{E}-02$ & $5 \mathrm{E}-02$ & E-02 & $9.72 \mathrm{E}-02$ & $30 \mathrm{E}-02$ \\
\hline Ba-137m & $6.27 \mathrm{E}-02$ & E-02 & $6.51 \mathrm{E}-02$ & $5 \mathrm{E}-02$ & $1.48 \mathrm{E}-01$ & -03 & 5.17 & $2.69 \mathrm{E}-01$ & $6 \mathrm{E}-01$ & $7.36 \mathrm{E}-03$ & $3.60 \mathrm{E}-02$ & $7.62 \mathrm{E}-02$ & $7.24 \mathrm{E}-02$ & $9.20 \mathrm{E}-02$ & $7 \mathrm{E}-02$ \\
\hline Sr-90 & $6.74 \mathrm{E}-02$ & $6.74 \mathrm{E}-02$ & $6.74 \mathrm{E}-02$ & $6.75 \mathrm{E}-02$ & $6.75 \mathrm{E}-02$ & & 6.75 & $6.75 \mathrm{E}-02$ & $5 \mathrm{E}-02$ & $4 \mathrm{E}-02$ & $4 \mathrm{E}-02$ & $6.74 \mathrm{E}-02$ & 4E-02 & $6.74 \mathrm{E}-02$ & $4 \mathrm{E}-02$ \\
\hline Y-90 & $6.74 \mathrm{E}-02$ & $6.74 \mathrm{E}-02$ & $6.74 \mathrm{E}-02$ & $6.75 \mathrm{E}-02$ & $6.75 \mathrm{E}-02$ & $6.75 \mathrm{E}-02$ & $6.75 \mathrm{E}-02$ & $6.75 \mathrm{E}-02$ & $6.75 \mathrm{E}-02$ & $6.74 \mathrm{E}-02$ & $6.74 \mathrm{E}-02$ & $6.74 \mathrm{E}-02$ & $6.74 \mathrm{E}-02$ & $6.74 \mathrm{E}-02$ & $6.74 \mathrm{E}-02$ \\
\hline $\mathrm{H}-3$ & -- & -- & 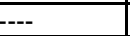 & -- & -- & -- & -- & -- & -- & --- & --- & -- & -- & -- & -- \\
\hline Ce-144 & 3.70 & 3.7 & 3.70 & 3.70 & $3.70 \mathrm{E}-06$ & & 3.70 & E-06 & $E-06$ & $E-06$ & & $\mathrm{E}-06$ & $3.70 \mathrm{E}-06$ & E-06 & $3.70 \mathrm{E}-06$ \\
\hline Pr-144 & $E-06$ & $\mathrm{E}-06$ & $3.70 \mathrm{E}-06$ & $\mathrm{E}-06$ & $3.70 \mathrm{E}-06$ & E-06 & 3.70 & $3.70 \mathrm{E}-06$ & $0 \mathrm{E}-06$ & $0 \mathrm{E}-06$ & DE-06 & $0 \mathrm{E}-06$ & DE-06 & $3.70 \mathrm{E}-06$ & $0 \mathrm{E}-06$ \\
\hline Pm-147 & $3.88 \mathrm{E}-03$ & $3.88 \mathrm{E}-03$ & $3.88 \mathrm{E}-03$ & $3.88 \mathrm{E}-03$ & $3.88 \mathrm{E}-03$ & 8E-03 & 3.88 & $8 \mathrm{E}-03$ & $8 \mathrm{E}-03$ & $\mathrm{E}-03$ & $3 \mathrm{E}-03$ & $3 \mathrm{E}-03$ & $3.88 \mathrm{E}-03$ & $3.88 \mathrm{E}-03$ & $3.88 \mathrm{E}-03$ \\
\hline Eu-154 & $7.56 \mathrm{E}-04$ & $7.56 \mathrm{E}-04$ & $7.56 \mathrm{E}-04$ & $7.56 \mathrm{E}-04$ & $7.56 \mathrm{E}-04$ & $7.56 \mathrm{E}-04$ & $7.56 \mathrm{E}-04$ & $7.56 \mathrm{E}-04$ & $7.56 \mathrm{E}-04$ & $7.56 \mathrm{E}-04$ & $7.56 \mathrm{E}-04$ & $7.56 \mathrm{E}-04$ & $7.56 \mathrm{E}-04$ & $7.56 \mathrm{E}-04$ & $7.56 \mathrm{E}-04$ \\
\hline Th-232 & $2.17 \mathrm{E}-09$ & $2.17 \mathrm{E}-09$ & $2.17 \mathrm{E}-09$ & $2.18 \mathrm{E}-09$ & $2.18 \mathrm{E}-09$ & & $2.17 \mathrm{E}-09$ & $2.18 \mathrm{E}-09$ & $2.18 \mathrm{E}-09$ & $2.17 \mathrm{E}-09$ & & $2.17 \mathrm{E}-09$ & $2.17 \mathrm{E}-09$ & $2.17 \mathrm{E}-09$ & $2.17 \mathrm{E}-09$ \\
\hline $\mathrm{U}-232$ & $E-10$ & $E-10$ & $\mathrm{E}-10$ & $E-10$ & $4.70 \mathrm{E}-10$ & $E-09$ & 1.15 & $E-10$ & $\mathrm{E}-10$ & $E-10$ & $\mathrm{E}-10$ & $E-10$ & $E-10$ & $3 \mathrm{E}-10$ & $5.19 \mathrm{E}-10$ \\
\hline U-233 & $E-08$ & E-08 & $7.35 \mathrm{E}-08$ & $\mathrm{E}-08$ & $7.49 \mathrm{E}-08$ & E-08 & 7.3 & $7.49 \mathrm{E}-08$ & $7.49 \mathrm{E}-08$ & E-08 & E-08 & $5 \mathrm{E}-08$ & E-08 & $7.35 \mathrm{E}-08$ & $7.35 \mathrm{E}-08$ \\
\hline $\mathrm{U}-234$ & $2.35 \mathrm{E}-08$ & $2.35 \mathrm{E}-08$ & $2.35 \mathrm{E}-08$ & $7 \mathrm{E}-08$ & $2.37 \mathrm{E}-08$ & $5 \mathrm{E}-08$ & 2.3 & $2.37 \mathrm{E}-08$ & $2.37 \mathrm{E}-08$ & E-08 & $2.69 \mathrm{E}-08$ & $2.35 \mathrm{E}-08$ & $35 \mathrm{E}-08$ & $2.35 \mathrm{E}-08$ & $2.35 \mathrm{E}-08$ \\
\hline $\mathrm{U}-235$ & $1.27 \mathrm{E}-09$ & $1.27 \mathrm{E}-09$ & $1.27 \mathrm{E}-09$ & $1.17 \mathrm{E}-09$ & $1.17 \mathrm{E}-09$ & 1.97E-09 & $1.79 \mathrm{E}-09$ & $1.17 \mathrm{E}-09$ & 1.17E-09 & $1.20 \mathrm{E}-09$ & $1.20 \mathrm{E}-09$ & $1.27 \mathrm{E}-09$ & $1.27 \mathrm{E}-09$ & $1.27 \mathrm{E}-09$ & $1.27 \mathrm{E}-09$ \\
\hline U-236 & $3.34 \mathrm{E}-09$ & 3.34E-09 & 3.34E-09 & 3.36E-09 & $3.36 \mathrm{E}-09$ & 3.34E-09 & $3.34 \mathrm{E}-09$ & $3.36 \mathrm{E}-09$ & $3.36 \mathrm{E}-09$ & 3.87E-09 & $3.86 \mathrm{E}-09$ & $3.34 \mathrm{E}-09$ & $3.34 \mathrm{E}-09$ & $3.34 \mathrm{E}-09$ & 3.34E-09 \\
\hline $\mathrm{U}-238$ & & & & & $E-08$ & & & & & & & -08 & & $\mathrm{E}-08$ & $0 \mathrm{E}-08$ \\
\hline Np-237 & & & $E-08$ & 5.1 & $\mathrm{E}-08$ & & 8.5 & $E-08$ & $E-08$ & $E-08$ & $E-08$ & E-08 & $\mathrm{E}-08$ & $5.08 \mathrm{E}-08$ & $8 \mathrm{E}-08$ \\
\hline Pu-238 & $1.52 \mathrm{E}-03$ & $2 \mathrm{E}-03$ & $1.52 \mathrm{E}-03$ & $3 \mathrm{E}-03$ & $1.43 \mathrm{E}-03$ & BE-03 & 1.43 & $1.43 \mathrm{E}-03$ & $1.43 \mathrm{E}-03$ & $3 \mathrm{E}-03$ & $3 \mathrm{E}-03$ & $1.52 \mathrm{E}-03$ & 22E-03 & $1.52 \mathrm{E}-03$ & $1.52 \mathrm{E}-03$ \\
\hline Pu-239 & $3.68 \mathrm{E}-05$ & $3.68 \mathrm{E}-05$ & $3.68 \mathrm{E}-05$ & $2.46 \mathrm{E}-05$ & $2.46 \mathrm{E}-05$ & $3.34 \mathrm{E}-05$ & $3.18 \mathrm{E}-05$ & $2.46 \mathrm{E}-05$ & $2.46 \mathrm{E}-05$ & $2.49 \mathrm{E}-05$ & $2.49 \mathrm{E}-05$ & $3.67 \mathrm{E}-05$ & $3.68 \mathrm{E}-05$ & 3.67E-05 & $3.69 \mathrm{E}-05$ \\
\hline Pu-240 & $1.42 \mathrm{E}-05$ & $1.41 \mathrm{E}-05$ & $1.42 \mathrm{E}-05$ & $1.14 \mathrm{E}-05$ & $1.14 \mathrm{E}-05$ & $1.34 \mathrm{E}-05$ & $1.30 \mathrm{E}-05$ & $1.14 \mathrm{E}-05$ & $1.14 \mathrm{E}-05$ & $1.16 \mathrm{E}-05$ & $1.16 \mathrm{E}-05$ & $1.41 \mathrm{E}-05$ & $1 \mathrm{E}-05$ & $1.41 \mathrm{E}-05$ & $1.42 \mathrm{E}-05$ \\
\hline Pu-241 & & & $8.53 \mathrm{E}-04$ & $E-04$ & $7.81 \mathrm{E}-04$ & & & & & & & $\mathrm{E}-04$ & -04 & E-04 & $4 \mathrm{E}-04$ \\
\hline Pu-242 & & & & & & & & & & & & & $9 \mathrm{E}-08$ & $2.29 \mathrm{E}-08$ & $2.29 \mathrm{E}-08$ \\
\hline Am-241 & $1.62 \mathrm{E}-04$ & $1.62 \mathrm{E}-04$ & $1.62 \mathrm{E}-04$ & $1.62 \mathrm{E}-04$ & $1.62 \mathrm{E}-04$ & $2.54 \mathrm{E}-04$ & $2.54 \mathrm{E}-04$ & $1.62 \mathrm{E}-04$ & $1.62 \mathrm{E}-04$ & $\mathrm{E}-04$ & $1.62 \mathrm{E}-04$ & $1.62 \mathrm{E}-04$ & $1.62 \mathrm{E}-04$ & $1.62 \mathrm{E}-04$ & $1.62 \mathrm{E}-04$ \\
\hline$A m-242 m$ & $1.52 \mathrm{E}-07$ & $1.52 \mathrm{E}-07$ & $1.52 \mathrm{E}-07$ & $1.53 \mathrm{E}-07$ & $1.53 \mathrm{E}-07$ & $2.78 \mathrm{E}-07$ & $2.78 \mathrm{E}-07$ & $1.53 \mathrm{E}-07$ & $1.53 \mathrm{E}-07$ & $1.52 \mathrm{E}-07$ & $1.52 \mathrm{E}-07$ & $1.52 \mathrm{E}-07$ & $1.52 \mathrm{E}-07$ & $1.52 \mathrm{E}-07$ & $1.52 \mathrm{E}-07$ \\
\hline$\overline{C m-242}$ & --- & 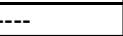 & 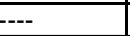 & --- & -- & -- & $-\overline{-}^{-}$ & ---- & --- & $-\overline{-}^{-}$ & --- & -- & ---- & --- & --. \\
\hline Cm-244 & $1.66 \mathrm{E}-04$ & $1.66 \mathrm{E}-04$ & $1.66 \mathrm{E}-04$ & $\begin{array}{l}1.66 \mathrm{E}-04 \\
\end{array}$ & $1.66 \mathrm{E}-04$ & $1.66 \mathrm{E}-04$ & $1.66 \mathrm{E}-04$ & 1.66E-04 & $1.66 \mathrm{E}-04$ & $1.66 \mathrm{E}-04$ & $1.66 \mathrm{E}-04$ & $1.66 \mathrm{E}-04$ & 1.66E-04 & $1.66 \mathrm{E}-04$ & $1.66 \mathrm{E}-04$ \\
\hline Cm-245 & $1.24 \mathrm{E}-08$ & $1.24 \mathrm{E}-08$ & $1.24 \mathrm{E}-08$ & $1.24 \mathrm{E}-08$ & $1.24 \mathrm{E}-08$ & $1.24 \mathrm{E}-08$ & $1.24 \mathrm{E}-08$ & $1.24 \mathrm{E}-08$ & $1.24 \mathrm{E}-08$ & $4 \mathrm{E}-08$ & $1.24 \mathrm{E}-08$ & $1.24 \mathrm{E}-08$ & E-08 & $1.24 \mathrm{E}-08$ & $1.24 \mathrm{E}-08$ \\
\hline Total Alpha & $1.90 \mathrm{E}-03$ & $1.89 \mathrm{E}-03$ & $1.89 \mathrm{E}-03$ & $1.80 \mathrm{E}-03$ & $1.80 \mathrm{E}-03$ & $1.90 \mathrm{E}-03$ & $1.90 \mathrm{E}-03$ & $1.80 \mathrm{E}-03$ & $1.80 \mathrm{E}-03$ & $1.89 \mathrm{E}-03$ & $1.89 \mathrm{E}-03$ & $1.89 \mathrm{E}-03$ & $1.89 \mathrm{E}-03$ & $1.89 \mathrm{E}-03$ & $1.90 \mathrm{E}-03$ \\
\hline
\end{tabular}


Table 9: Projected Feed Composition After Dissolution and Dilution to 6.44 M Total Sodium Concentration $350 \mathrm{mg} / \mathrm{L}$ sludge; $20 \%$ of interstitial supernate remaining

\begin{tabular}{|c|c|c|c|c|c|c|c|c|c|c|c|c|c|c|c|}
\hline \multicolumn{16}{|c|}{ Concentration (M) } \\
\hline & Tank 25 & Tank 27 & Tank 28 & Tank 29 & Tank 31 & Tank 33 & Tank 34 & Tank 36 & Tank 37 & Tank 38 & Tank 41 & Tank 44 & Tank 45 & Tank 46 & Tank 47 \\
\hline $\mathrm{Na}$ & $6.44 \mathrm{E}+00$ & $6.44 \mathrm{E}+00$ & $6.44 \mathrm{E}+00$ & $6.44 \mathrm{E}+00$ & $6.44 \mathrm{E}+00$ & $6.44 \mathrm{E}+00$ & $6.44 \mathrm{E}+00$ & $6.44 \mathrm{E}+00$ & $6.44 \mathrm{E}+00$ & $6.44 \mathrm{E}+00$ & $6.44 \mathrm{E}+00$ & $6.44 \mathrm{E}+00$ & $6.44 \mathrm{E}+00$ & $6.44 \mathrm{E}+00$ & $6.44 \mathrm{E}+00$ \\
\hline$\overline{\mathrm{Al}(\mathrm{OH}) 4}$ & $3.21 \mathrm{E}-01$ & $3.29 \mathrm{E}-01$ & $3.21 \mathrm{E}-01$ & $3.99 \mathrm{E}-01$ & $4.00 \mathrm{E}-01$ & $3.20 \mathrm{E}-01$ & $3.19 \mathrm{E}-01$ & $3.89 \mathrm{E}-01$ & $3.95 \mathrm{E}-01$ & $4.00 \mathrm{E}-01$ & $3.94 \mathrm{E}-01$ & $3.18 \mathrm{E}-01$ & $3.16 \mathrm{E}-01$ & $3.12 \mathrm{E}-01$ & $3.22 \mathrm{E}-01$ \\
\hline $\mathrm{CO} 3$ & $2.20 \mathrm{E}-01$ & $2.19 \mathrm{E}-01$ & $2.18 \mathrm{E}-01$ & 1.33E-01 & $1.31 \mathrm{E}-01$ & $2.23 \mathrm{E}-01$ & $2.26 \mathrm{E}-01$ & $1.31 \mathrm{E}-01$ & $1.31 \mathrm{E}-01$ & $1.35 \mathrm{E}-01$ & $1.34 \mathrm{E}-01$ & $2.20 \mathrm{E}-01$ & $2.22 \mathrm{E}-01$ & $2.17 \mathrm{E}-01$ & $2.24 \mathrm{E}-01$ \\
\hline$\overline{\mathrm{C2O4}}$ & $2.21 \mathrm{E}-02$ & $2.20 \mathrm{E}-02$ & $2.20 \mathrm{E}-02$ & $1.41 \mathrm{E}-02$ & $1.39 \mathrm{E}-02$ & $2.25 \mathrm{E}-02$ & $2.21 \mathrm{E}-02$ & $1.39 \mathrm{E}-02$ & $1.40 \mathrm{E}-02$ & $1.42 \mathrm{E}-02$ & $1.40 \mathrm{E}-02$ & $2.19 \mathrm{E}-02$ & $2.20 \mathrm{E}-02$ & $2.19 \mathrm{E}-02$ & $2.22 \mathrm{E}-02$ \\
\hline NO2 & $1.19 \mathrm{E}-01$ & $1.20 \mathrm{E}-01$ & $1.24 \mathrm{E}-01$ & $7.46 \mathrm{E}-01$ & $7.56 \mathrm{E}-01$ & $1.02 \mathrm{E}-01$ & $1.17 \mathrm{E}-01$ & 7.52E-01 & $7.48 \mathrm{E}-01$ & $7.44 \mathrm{E}-01$ & $7.40 \mathrm{E}-01$ & $1.21 \mathrm{E}-01$ & $1.24 \mathrm{E}-01$ & $1.22 \mathrm{E}-01$ & $1.09 \mathrm{E}-01$ \\
\hline NO3 & $3.76 \mathrm{E}+00$ & $3.75 \mathrm{E}+00$ & $3.76 \mathrm{E}+00$ & $2.65 \mathrm{E}+00$ & $2.63 \mathrm{E}+00$ & $3.83 \mathrm{E}+00$ & $3.78 \mathrm{E}+00$ & $2.62 \mathrm{E}+00$ & $2.63 \mathrm{E}+00$ & $2.67 \mathrm{E}+00$ & $2.66 \mathrm{E}+00$ & $3.74 \mathrm{E}+00$ & $3.74 \mathrm{E}+00$ & $3.74 \mathrm{E}+00$ & $3.80 \mathrm{E}+00$ \\
\hline$\overline{\mathrm{OH}}$ & $1.02 \mathrm{E}+00$ & $1.02 \mathrm{E}+00$ & $9.85 \mathrm{E}-01$ & $1.85 \mathrm{E}+00$ & $1.87 \mathrm{E}+00$ & $9.20 \mathrm{E}-01$ & $9.75 \mathrm{E}-01$ & $1.94 \mathrm{E}+00$ & $1.89 \mathrm{E}+00$ & $1.86 \mathrm{E}+00$ & $1.86 \mathrm{E}+00$ & $1.05 \mathrm{E}+00$ & $1.07 \mathrm{E}+00$ & $9.69 \mathrm{E}-01$ & $9.45 \mathrm{E}-01$ \\
\hline$\overline{\mathrm{PO} 4}$ & $1.53 \mathrm{E}-03$ & 1.53E-03 & $1.62 \mathrm{E}-03$ & 1.57E-02 & $1.56 \mathrm{E}-02$ & $1.37 \mathrm{E}-03$ & $1.37 \mathrm{E}-03$ & 1.57E-02 & 1.55E-02 & $1.57 \mathrm{E}-02$ & 1.57E-02 & $1.44 \mathrm{E}-03$ & $1.45 \mathrm{E}-03$ & $1.51 \mathrm{E}-03$ & $1.37 \mathrm{E}-03$ \\
\hline SO4 & $3.21 \mathrm{E}-01$ & $3.20 \mathrm{E}-01$ & $3.21 \mathrm{E}-01$ & $1.83 \mathrm{E}-01$ & $1.80 \mathrm{E}-01$ & $3.28 \mathrm{E}-01$ & $3.22 \mathrm{E}-01$ & $1.80 \mathrm{E}-01$ & $1.80 \mathrm{E}-01$ & $1.85 \mathrm{E}-01$ & $1.82 \mathrm{E}-01$ & $3.19 \mathrm{E}-01$ & $3.20 \mathrm{E}-01$ & $3.19 \mathrm{E}-01$ & $3.24 \mathrm{E}-01$ \\
\hline $\mathbf{A g}$ & $1.21 \mathrm{E}-08$ & $7.10 \mathrm{E}-08$ & $5.23 \mathrm{E}-08$ & $2.65 \mathrm{E}-08$ & $2.26 \mathrm{E}-08$ & $2.49 \mathrm{E}-08$ & $2.45 \mathrm{E}-08$ & $2.26 \mathrm{E}-08$ & $2.26 \mathrm{E}-08$ & $2.92 \mathrm{E}-08$ & $2.27 \mathrm{E}-08$ & $2.43 \mathrm{E}-08$ & $2.44 \mathrm{E}-08$ & $2.43 \mathrm{E}-08$ & $2.46 \mathrm{E}-08$ \\
\hline As & $2.13 \mathrm{E}-06$ & $1.93 \mathrm{E}-06$ & $2.51 \mathrm{E}-06$ & $3.45 \mathrm{E}-06$ & $3.55 \mathrm{E}-05$ & $1.72 \mathrm{E}-05$ & $4.37 \mathrm{E}-05$ & $6.17 \mathrm{E}-05$ & $4.44 \mathrm{E}-05$ & $2.38 \mathrm{E}-06$ & $2.94 \mathrm{E}-05$ & $7.38 \mathrm{E}-05$ & $8.09 \mathrm{E}-05$ & $4.45 \mathrm{E}-05$ & $3.08 \mathrm{E}-05$ \\
\hline $\mathrm{Ba}$ & $2.11 \mathrm{E}-09$ & E-08 & $1.58 \mathrm{E}-08$ & 1.39E-07 & -- & -- & -- & -- & -- & E-09 & -- & --- & -- & --- & - \\
\hline $\mathrm{Ca}$ & $4.98 \mathrm{E}-05$ & $4.97 \mathrm{E}-05$ & $4.98 \mathrm{E}-05$ & $3.17 \mathrm{E}-05$ & $3.05 \mathrm{E}-05$ & $5.19 \mathrm{E}-05$ & 4.99 & $3.05 \mathrm{E}-05$ & $3.05 \mathrm{E}-05$ & E-05 & E-01 & E-05 & $4.97 \mathrm{E}-05$ & $4.95 \mathrm{E}-05$ & $5.04 \mathrm{E}-05$ \\
\hline Cd & -08 & -08 & $E-08$ & $\mathrm{E}-08$ & $9.57 \mathrm{E}-07$ & $2.06 \mathrm{E}-07$ & & $1.97 \mathrm{E}-06$ & $1.30 \mathrm{E}-06$ & E-08 & E-07 & E-06 & $2.68 \mathrm{E}-06$ & $1.27 \mathrm{E}-06$ & 7.37E-07 \\
\hline$\overline{\mathrm{Cl}}$ & $4.23 \mathrm{E}-02$ & $4.22 \mathrm{E}-02$ & $4.23 \mathrm{E}-02$ & $3.84 \mathrm{E}-02$ & $3.79 \mathrm{E}-02$ & $4.29 \mathrm{E}-02$ & BE-02 & $3.80 \mathrm{E}-02$ & $3.79 \mathrm{E}-02$ & $3.87 \mathrm{E}-02$ & $3.81 \mathrm{E}-02$ & $4.21 \mathrm{E}-02$ & $4.23 \mathrm{E}-02$ & $4.20 \mathrm{E}-02$ & $4.24 \mathrm{E}-02$ \\
\hline Co & $3.09 \mathrm{E}-07$ & $3.15 \mathrm{E}-07$ & $2.60 \mathrm{E}-07$ & $1.37 \mathrm{E}-07$ & $2.02 \mathrm{E}-07$ & $1.32 \mathrm{E}-07$ & $2.41 \mathrm{E}-07$ & $3.11 \mathrm{E}-07$ & $2.39 \mathrm{E}-07$ & $1.33 \mathrm{E}-07$ & 1.77E-07 & $3.65 \mathrm{E}-07$ & $3.95 \mathrm{E}-07$ & $2.44 \mathrm{E}-07$ & $1.88 \mathrm{E}-07$ \\
\hline $\mathrm{Cr}$ & $1.28 \mathrm{E}-05$ & $7.79 \mathrm{E}-06$ & $2.59 \mathrm{E}-05$ & $1.06 \mathrm{E}-04$ & $1.42 \mathrm{E}-04$ & $1.75 \mathrm{E}-04$ & $1.50 \mathrm{E}-04$ & $1.21 \mathrm{E}-04$ & $1.35 \mathrm{E}-04$ & $1.34 \mathrm{E}-04$ & $1.48 \mathrm{E}-04$ & $1.24 \mathrm{E}-04$ & $1.19 \mathrm{E}-04$ & $1.48 \mathrm{E}-04$ & $1.61 \mathrm{E}-04$ \\
\hline $\mathrm{Cu}$ & $1.11 \mathrm{E}-04$ & $1.15 \mathrm{E}-04$ & $8.39 \mathrm{E}-05$ & $2.00 \mathrm{E}-05$ & $5.68 \mathrm{E}-05$ & $1.23 \mathrm{E}-05$ & -05 & 1.17E-04 & $7.71 \mathrm{E}-05$ & E-05 & $5-05$ & $1.42 \mathrm{E}-04$ & $1.59 \mathrm{E}-04$ & 7.54E-05 & $4.38 \mathrm{E}-05$ \\
\hline $\mathrm{Fe}$ & 07 & -07 & $7.26 \mathrm{E}-07$ & $5.60 \mathrm{E}-07$ & $2.58 \mathrm{E}-06$ & $6.30 \mathrm{E}-07$ & 3.3 & $5.23 \mathrm{E}-06$ & $3.48 \mathrm{E}-06$ & E-06 & E-06 & E-06 & $7.08 \mathrm{E}-06$ & $3.41 \mathrm{E}-06$ & $2.02 \mathrm{E}-06$ \\
\hline $\mathbf{F}$ & $7.04 \mathrm{E}-02$ & $7.02 \mathrm{E}-02$ & $7.03 \mathrm{E}-02$ & $3.61 \mathrm{E}-02$ & $3.56 \mathrm{E}-02$ & $7.17 \mathrm{E}-02$ & 7.0 & $3.56 \mathrm{E}-02$ & $3.56 \mathrm{E}-02$ & $3.63 \mathrm{E}-02$ & $3.57 \mathrm{E}-02$ & $7.00 \mathrm{E}-02$ & $7.02 \mathrm{E}-02$ & $6.98 \mathrm{E}-02$ & 7.09E-02 \\
\hline $\mathrm{Hg}$ & $7.23 \mathrm{E}-07$ & $7.21 \mathrm{E}-07$ & $7.22 \mathrm{E}-07$ & $1.36 \mathrm{E}-06$ & $8.20 \mathrm{E}-06$ & $8.61 \mathrm{E}-06$ & $E-06$ & $8.74 \mathrm{E}-06$ & $8.38 \mathrm{E}-06$ & E-07 & E-06 & $9.57 \mathrm{E}-06$ & $9.73 \mathrm{E}-06$ & $8.95 \mathrm{E}-06$ & $8.79 \mathrm{E}-06$ \\
\hline $\mathrm{K}$ & $6.61 \mathrm{E}-03$ & $6.74 \mathrm{E}-03$ & $6.60 \mathrm{E}-03$ & $4.99 \mathrm{E}-03$ & $5.38 \mathrm{E}-03$ & $5.01 \mathrm{E}-03$ & $5.84 \mathrm{E}-03$ & $6.64 \mathrm{E}-03$ & $5.53 \mathrm{E}-03$ & $4.65 \mathrm{E}-03$ & $4.88 \mathrm{E}-03$ & $7.01 \mathrm{E}-03$ & $7.31 \mathrm{E}-03$ & $5.93 \mathrm{E}-03$ & $5.55 \mathrm{E}-03$ \\
\hline $\mathrm{Mg}$ & 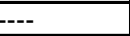 & --- & $6.80 \mathrm{E}-09$ & $8.76 \mathrm{E}-08$ & $3.49 \mathrm{E}-08$ & $1.08 \mathrm{E}-07$ & $2.19 \mathrm{E}-08$ & ---- & $6.92 \mathrm{E}-09$ & $9.24 \mathrm{E}-08$ & $5.53 \mathrm{E}-08$ & ---- & $\begin{array}{ll}--- \\
\end{array}$ & $1.78 \mathrm{E}-08$ & $6.32 \mathrm{E}-08$ \\
\hline Mn & $3.96 \mathrm{E}-08$ & $4.74 \mathrm{E}-08$ & $6.32 \mathrm{E}-08$ & $7.43 \mathrm{E}-08$ & $1.34 \mathrm{E}-07$ & $6.99 \mathrm{E}-08$ & $1.63 \mathrm{E}-07$ & $2.26 \mathrm{E}-07$ & $1.65 \mathrm{E}-07$ & $3.99 \mathrm{E}-08$ & $1.12 \mathrm{E}-07$ & \begin{tabular}{|c|}
$2.69 \mathrm{E}-07$ \\
\end{tabular} & \begin{tabular}{|c|}
$2.94 \mathrm{E}-07$ \\
\end{tabular} & $1.66 \mathrm{E}-07$ & $1.18 \mathrm{E}-07$ \\
\hline Nd & --- & ---- & --- & $2.91 \mathrm{E}-06$ & -- & $3.91 \mathrm{E}-06$ & -- & --- & -- & $E-06$ & E-06 & --- & ---- & ---- & $1.35 \mathrm{E}-06$ \\
\hline $\mathrm{Ni}$ & $7.40 \mathrm{E}-07$ & 7.57E-07 & 6.09E-07 & $2.88 \mathrm{E}-07$ & $4.63 \mathrm{E}-07$ & $2.68 \mathrm{E}-07$ & 5.59E-07 & 7.52E-07 & 5.61E-07 & -07 & $3.95 \mathrm{E}-07$ & $8.91 \mathrm{E}-07$ & 9.69E-07 & 5.67E-07 & $4.17 \mathrm{E}-07$ \\
\hline $\mathrm{Pb}$ & $2.03 \mathrm{E}-06$ & 1.67E-06 & $2.31 \mathrm{E}-06$ & $3.35 \mathrm{E}-06$ & --- & --- & --- & \begin{tabular}{l|l}
--- \\
\end{tabular} & -- & $1.98 \mathrm{E}-06$ & -- & --- & --- & $-\cdots$ & - \\
\hline$\overline{R u}$ & $1.17 \mathrm{E}-05$ & $1.21 \mathrm{E}-05$ & 9.07E-06 & $2.78 \mathrm{E}-06$ & $6.36 \mathrm{E}-06$ & $2.10 \mathrm{E}-06$ & $8.04 \mathrm{E}-06$ & $1.22 \mathrm{E}-05$ & $8.34 \mathrm{E}-06$ & $2.51 \mathrm{E}-06$ & 4.97E-06 & $1.48 \mathrm{E}-05$ & $1.64 \mathrm{E}-05$ & $8.24 \mathrm{E}-06$ & $5.16 \mathrm{E}-06$ \\
\hline $\mathrm{Se}$ & $1.51 \mathrm{E}-05$ & $1.15 \mathrm{E}-05$ & $1.94 \mathrm{E}-05$ & $4.05 \mathrm{E}-05$ & $1.09 \mathrm{E}-04$ & $1.46 \mathrm{E}-04$ & $1.13 \mathrm{E}-04$ & $7.90 \mathrm{E}-05$ & $9.89 \mathrm{E}-05$ & $3.90 \mathrm{E}-05$ & $1.17 \mathrm{E}-04$ & $7.68 \mathrm{E}-05$ & $6.90 \mathrm{E}-05$ & $1.10 \mathrm{E}-04$ & $1.28 \mathrm{E}-04$ \\
\hline$\underline{\mathrm{Si}}$ & $7.17 \mathrm{E}-05$ & $6.28 \mathrm{E}-05$ & $3.68 \mathrm{E}-04$ & $1.21 \mathrm{E}-05$ & --- & --- & $\cdots$ & --- & ---- & $9.51 \mathrm{E}-05$ & --- & 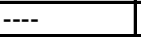 & ---- & $-\cdots$ & $-\overline{-}$ \\
\hline $\mathrm{Sr}$ & --- & ---- & ---- & $1.96 \mathrm{E}-06$ & --- & $2.71 \mathrm{E}-06$ & $\cdots$ & ---- & ---- & $2.16 \mathrm{E}-06$ & 5.35E-07 & ---- & ---- & ---- & $7.27 \mathrm{E}-07$ \\
\hline $\mathrm{Zn}$ & $1.35 \mathrm{E}-04$ & 1.39E-04 & 1.05E-04 & $3.51 \mathrm{E}-05$ & $7.48 \mathrm{E}-05$ & $2.79 \mathrm{E}-05$ & $9.39 \mathrm{E}-05$ & $1.40 \mathrm{E}-04$ & $9.69 \mathrm{E}-05$ & $3.20 \mathrm{E}-05$ & 5.94E-05 & $1.69 \mathrm{E}-04$ & $1.86 \mathrm{E}-04$ & $9.61 \mathrm{E}-05$ & $6.19 \mathrm{E}-05$ \\
\hline$Z r$ & 3.55E-06 & $3.66 \mathrm{E}-06$ & $2.70 \mathrm{E}-06$ & 7.09E-07 & $1.85 \mathrm{E}-06$ & $4.76 \mathrm{E}-07$ & 2.37E-06 & 3.72E-06 & $2.48 \mathrm{E}-06$ & 6.19E-07 & $1.41 \mathrm{E}-06$ & $4.52 \mathrm{E}-06$ & $5.03 E-06$ & $2.44 \mathrm{E}-06$ & $1.46 \mathrm{E}-06$ \\
\hline Insol.Sol.mg/L & 350 & 350 & 350 & 350 & 350 & 350 & 350 & 350 & 350 & 350 & 350 & 350 & 350 & 350 & 350 \\
\hline
\end{tabular}


Table 9: Projected Feed Composition After Dissolution and Dilution to 6.44 M Total Sodium Concentration $350 \mathrm{mg} / \mathrm{L}$ sludge; $20 \%$ of interstitial supernate remaining (continued)

\begin{tabular}{|c|c|c|c|c|c|c|c|c|c|c|c|c|c|c|c|}
\hline \multicolumn{16}{|c|}{ Radionuclide Composition (Ci/gal) } \\
\hline & Tank 25 & Tank 27 & Tank 28 & Tank 29 & Tank 31 & Tank 33 & Tank 34 & Tank 36 & Tank 37 & Tank 38 & Tank 41 & Tank 44 & Tank 45 & Tank 46 & Tank 47 \\
\hline C-14 & $1.97 \mathrm{E}-09$ & $1.97 \mathrm{E}-09$ & $1.97 \mathrm{E}-09$ & $1.97 \mathrm{E}-09$ & $1.97 \mathrm{E}-09$ & $1.97 \mathrm{E}-09$ & $1.97 \mathrm{E}-09$ & $1.97 \mathrm{E}-09$ & 1.97E-09 & 1.97E-09 & $1.97 \mathrm{E}-09$ & $1.97 \mathrm{E}-09$ & $1.97 \mathrm{E}-09$ & $1.97 \mathrm{E}-09$ & $1.97 \mathrm{E}-09$ \\
\hline Ni-59 & $1.13 \mathrm{E}-06$ & $1.13 \mathrm{E}-06$ & $1.13 \mathrm{E}-06$ & $1.13 \mathrm{E}-06$ & $1.13 \mathrm{E}-06$ & $1.13 \mathrm{E}-06$ & $1.13 \mathrm{E}-06$ & $1.13 \mathrm{E}-06$ & $1.13 \mathrm{E}-06$ & $1.13 \mathrm{E}-06$ & $1.13 \mathrm{E}-06$ & $1.13 \mathrm{E}-06$ & $1.13 \mathrm{E}-06$ & $1.13 \mathrm{E}-06$ & $1.13 \mathrm{E}-06$ \\
\hline Ni-63 & 1.76E-09 & $1.76 \mathrm{E}-09$ & $1.76 \mathrm{E}-09$ & $1.76 \mathrm{E}-09$ & $1.76 \mathrm{E}-09$ & $1.76 \mathrm{E}-09$ & $1.76 \mathrm{E}-09$ & $1.76 \mathrm{E}-09$ & $1.76 \mathrm{E}-09$ & $1.76 \mathrm{E}-09$ & $1.76 \mathrm{E}-09$ & $1.76 \mathrm{E}-09$ & $1.76 \mathrm{E}-09$ & $1.76 \mathrm{E}-09$ & $1.76 \mathrm{E}-09$ \\
\hline Co-60 & $1.80 \mathrm{E}-04$ & $1.80 \mathrm{E}-04$ & $1.80 \mathrm{E}-04$ & $1.80 \mathrm{E}-04$ & $1.80 \mathrm{E}-04$ & $1.80 \mathrm{E}-04$ & $1.80 \mathrm{E}-04$ & $1.80 \mathrm{E}-04$ & $1.80 \mathrm{E}-04$ & $1.80 \mathrm{E}-04$ & $1.80 \mathrm{E}-04$ & $1.80 \mathrm{E}-04$ & $1.80 \mathrm{E}-04$ & $1.80 \mathrm{E}-04$ & 1.80E-04 \\
\hline Se-79 & 67E-07 & $6.67 \mathrm{E}-07$ & $6.67 \mathrm{E}-07$ & $6.67 \mathrm{E}-07$ & $6.67 \mathrm{E}-07$ & $6.67 \mathrm{E}-07$ & $6.67 \mathrm{E}-07$ & $6.67 \mathrm{E}-07$ & $6.67 \mathrm{E}-07$ & $6.67 \mathrm{E}-07$ & $6.67 \mathrm{E}-07$ & $6.67 \mathrm{E}-07$ & $6.67 \mathrm{E}-07$ & $6.67 \mathrm{E}-07$ & \\
\hline Tc-99 & $1.13 \mathrm{E}-05$ & $1.13 \mathrm{E}-05$ & $1.13 \mathrm{E}-05$ & $1.13 \mathrm{E}-05$ & $1.13 \mathrm{E}-05$ & $1.13 \mathrm{E}-05$ & $1.13 \mathrm{E}-05$ & $.13 \mathrm{E}-05$ & $1.13 \mathrm{E}-05$ & $1.13 \mathrm{E}-05$ & $1.13 \mathrm{E}-05$ & $1.13 \mathrm{E}-05$ & $1.13 \mathrm{E}-05$ & $1.13 \mathrm{E}-05$ & $1.13 \mathrm{E}-05$ \\
\hline Ru-106 & $4.19 \mathrm{E}-06$ & $4.19 \mathrm{E}-06$ & $4.19 \mathrm{E}-06$ & $4.19 \mathrm{E}-06$ & $4.19 \mathrm{E}-06$ & $4.19 \mathrm{E}-06$ & $4.19 \mathrm{E}-06$ & $4.19 \mathrm{E}-06$ & $4.19 \mathrm{E}-06$ & $4.19 \mathrm{E}-06$ & $4.19 \mathrm{E}-06$ & $4.19 \mathrm{E}-06$ & $4.19 \mathrm{E}-06$ & $4.19 \mathrm{E}-06$ & $4.19 \mathrm{E}-06$ \\
\hline Rh-106 & $4.19 \mathrm{E}-06$ & $4.19 \mathrm{E}-06$ & $4.19 \mathrm{E}-06$ & $4.19 \mathrm{E}-06$ & $4.19 \mathrm{E}-06$ & $4.19 \mathrm{E}-06$ & $4.19 \mathrm{E}-06$ & $4.19 \mathrm{E}-06$ & $4.19 \mathrm{E}-06$ & $4.19 \mathrm{E}-06$ & $4.19 \mathrm{E}-06$ & $4.19 \mathrm{E}-06$ & $4.19 \mathrm{E}-06$ & $4.19 \mathrm{E}-06$ & $4.19 \mathrm{E}-06$ \\
\hline Sb-125 & 1.07E-04 & 1.07E-04 & $1.07 \mathrm{E}-04$ & 1.07E-04 & 1.07E-04 & 1.07E-04 & 1.07E-04 & 1.07E-04 & 1.07E-04 & 1.07E-04 & $1.07 \mathrm{E}-04$ & 1.07E-04 & $1.07 \mathrm{E}-04$ & $1.07 \mathrm{E}-04$ & $1.07 \mathrm{E}-04$ \\
\hline Sn-126 & $8.92 \mathrm{E}-07$ & $8.92 \mathrm{E}-07$ & $8.92 \mathrm{E}-07$ & $8.92 \mathrm{E}-07$ & $8.92 \mathrm{E}-07$ & $8.92 \mathrm{E}-07$ & $8.92 \mathrm{E}-07$ & $8.92 \mathrm{E}-07$ & $8.92 \mathrm{E}-07$ & $8.92 \mathrm{E}-07$ & $8.92 \mathrm{E}-07$ & $8.92 \mathrm{E}-07$ & $8.92 \mathrm{E}-07$ & $8.92 \mathrm{E}-07$ & $8.92 \mathrm{E}-07$ \\
\hline |-129 & -10 & -10 & $E-10$ & $1.04 \mathrm{E}-10$ & -10 & & $E-10$ & & $E-10$ & $E-10$ & $\mathrm{E}-10$ & $E-10$ & $E-10$ & $1.04 \mathrm{E}-10$ & $.04 \mathrm{E}-10$ \\
\hline Cs-134 & -06 & -06 & $E-06$ & $4.70 \mathrm{E}-06$ & $\mathrm{E}-06$ & 4.7 & $E-06$ & -06 & E-06 & $\mathrm{E}-06$ & $4.70 \mathrm{E}-06$ & E-06 & E-06 & $4.70 \mathrm{E}-06$ & $0 \mathrm{E}-06$ \\
\hline Cs-135 & E-09 & 7.98 & $7.98 \mathrm{E}-09$ & $7.98 \mathrm{E}-09$ & $7.98 \mathrm{E}-09$ & 7.98 & E-09 & $E-09$ & $7.98 \mathrm{E}-09$ & $7.98 \mathrm{E}-09$ & $7.98 \mathrm{E}-09$ & $8 \mathrm{E}-09$ & $7.98 \mathrm{E}-09$ & $7.98 \mathrm{E}-09$ & $8 \mathrm{E}-09$ \\
\hline Cs-137 & $6.46 \mathrm{E}-02$ & $6.13 \mathrm{E}-02$ & $6.71 \mathrm{E}-02$ & $5.80 \mathrm{E}-02$ & $1.54 \mathrm{E}-01$ & & $9 \mathrm{E}-02$ & $2 \mathrm{E}-01$ & $6 \mathrm{E}-01$ & E-03 & E-02 & $\mathrm{E}-02$ & $8 \mathrm{E}-02$ & $9.55 \mathrm{E}-02$ & $3 \mathrm{E}-02$ \\
\hline Ba-137m & $6.11 \mathrm{E}-02$ & $5.80 \mathrm{E}-02$ & $6.35 \mathrm{E}-02$ & $5.49 \mathrm{E}-02$ & $1.46 \mathrm{E}-01$ & $3.64 \mathrm{E}-03$ & $5.01 \mathrm{E}-02$ & $2.67 \mathrm{E}-01$ & 1.94E-01 & $5.75 \mathrm{E}-03$ & $3.44 \mathrm{E}-02$ & $7.46 \mathrm{E}-02$ & $7.08 \mathrm{E}-02$ & $9.04 \mathrm{E}-02$ & $3.91 \mathrm{E}-02$ \\
\hline Sr-90 & $3.93 \mathrm{E}-02$ & 3.93E-02 & $3.93 \mathrm{E}-02$ & 3.94E-02 & $3.94 \mathrm{E}-02$ & & $3.94 \mathrm{E}-02$ & $3.94 \mathrm{E}-02$ & $3.94 \mathrm{E}-02$ & 3.93E-02 & 3.93E-02 & $3.93 \mathrm{E}-02$ & $3.93 \mathrm{E}-02$ & $3.93 \mathrm{E}-02$ & 3.93E-02 \\
\hline Y-90 & $3.93 \mathrm{E}-02$ & $3.93 \mathrm{E}-02$ & $3.93 \mathrm{E}-02$ & 3.94E-02 & 3.94E-02 & 3.94 & $4 \mathrm{E}-02$ & E-02 & $3.94 \mathrm{E}-02$ & $3.93 \mathrm{E}-02$ & $3.93 \mathrm{E}-02$ & $3.93 \mathrm{E}-02$ & $3.93 \mathrm{E}-02$ & $3.93 \mathrm{E}-02$ & 3.93E-02 \\
\hline $\mathrm{H}-3$ & -- & -- & $\cdots$ & -- & --- & --- & --- & --- & \begin{tabular}{l|l}
-- \\
-
\end{tabular} & --- & -- & -- & -- & - & - \\
\hline Ce-144 & 2.16 & 2.16 & 2.16 & $2.16 \mathrm{E}-06$ & 2.16 & & -06 & -06 & $\mathrm{E}-06$ & E-06 & $=-06$ & $E-06$ & E-06 & $2.16 \mathrm{E}-06$ & \\
\hline Pr-144 & -06 & -06 & $2.16 \mathrm{E}-06$ & $2.16 \mathrm{E}-06$ & E-06 & $=-06$ & E-06 & -06 & -06 & -06 & $=-06$ & -06 & $E-06$ & $E-06$ & E-06 \\
\hline Pm-147 & $2.26 \mathrm{E}-03$ & $2.26 \mathrm{E}-03$ & $2.26 \mathrm{E}-03$ & $2.26 \mathrm{E}-03$ & $2.26 \mathrm{E}-03$ & -03 & $E-03$ & $E-03$ & $2.26 \mathrm{E}-03$ & $2.26 \mathrm{E}-03$ & $2.26 \mathrm{E}-03$ & $2.26 \mathrm{E}-03$ & $2.26 \mathrm{E}-03$ & $2.26 \mathrm{E}-03$ & $2.26 \mathrm{E}-03$ \\
\hline Eu-154 & $4.41 \mathrm{E}-04$ & $4.41 \mathrm{E}-04$ & $4.41 \mathrm{E}-04$ & $4.41 \mathrm{E}-04$ & $4.41 \mathrm{E}-04$ & $4.41 \mathrm{E}-04$ & $4.41 \mathrm{E}-04$ & $4.41 \mathrm{E}-04$ & $4.41 \mathrm{E}-04$ & $4.41 \mathrm{E}-04$ & $4.41 \mathrm{E}-04$ & $4.41 \mathrm{E}-04$ & $4.41 \mathrm{E}-04$ & $4.41 \mathrm{E}-04$ & $4.41 \mathrm{E}-04$ \\
\hline Th-232 & $1.27 \mathrm{E}-09$ & $1.27 \mathrm{E}-09$ & $1.27 \mathrm{E}-09$ & $1.27 \mathrm{E}-09$ & $1.27 \mathrm{E}-09$ & & $1.27 \mathrm{E}-09$ & $1.27 \mathrm{E}-09$ & $1.27 \mathrm{E}-09$ & $1.27 \mathrm{E}-09$ & $1.27 \mathrm{E}-09$ & $1.27 \mathrm{E}-09$ & $1.27 \mathrm{E}-09$ & $1.27 \mathrm{E}-09$ & $1.27 \mathrm{E}-09$ \\
\hline $\mathrm{U}-232$ & & 3.24 & 3.24 & -10 & -10 & & & & -10 & -10 & -10 & & -10 & $E-10$ & $\mathrm{E}-10$ \\
\hline $\mathrm{U}-233$ & -08 & 4.29 & $4.29 \mathrm{E}-08$ & $4.44 \mathrm{E}-08$ & E-08 & 4.2 & E-08 & $E-08$ & E-08 & E-08 & E-08 & E-08 & E-08 & $4.29 \mathrm{E}-08$ & E-08 \\
\hline U-234 & -08 & 1.37 & $1.37 \mathrm{E}-08$ & 1.39E-08 & E-08 & 1.3 & E-08 & $E-08$ & $\mathrm{E}-08$ & $1.72 \mathrm{E}-08$ & $E-08$ & E-08 & $1.37 \mathrm{E}-08$ & 1.37E-08 & $7 \mathrm{E}-08$ \\
\hline U-235 & $=-10$ & 7.90 & $7.90 \mathrm{E}-10$ & $6.83 \mathrm{E}-10$ & $6.83 \mathrm{E}-10$ & $1.4 \varepsilon$ & $E-09$ & $E-10$ & $\mathrm{E}-10$ & $E-10$ & $\mathrm{E}-10$ & $\mathrm{E}-10$ & -10 & $\mathrm{E}-10$ & $\mathrm{E}-10$ \\
\hline $\mathrm{U}-236$ & $1.95 \mathrm{E}-09$ & $1.95 \mathrm{E}-09$ & $1.95 \mathrm{E}-09$ & $1.97 \mathrm{E}-09$ & 1.97E-09 & -09 & E-09 & E-09 & 1.97E-09 & $2.48 \mathrm{E}-09$ & $2.47 \mathrm{E}-09$ & $1.95 \mathrm{E}-09$ & $1.95 \mathrm{E}-09$ & $1.95 \mathrm{E}-09$ & $1.95 \mathrm{E}-09$ \\
\hline U-238 & $3.11 \mathrm{E}-08$ & $3.11 \mathrm{E}-08$ & $3.11 \mathrm{E}-08$ & $2.08 \mathrm{E}-08$ & $2.08 \mathrm{E}-08$ & -08 & -08 & $E-08$ & $2.08 \mathrm{E}-08$ & $E-08$ & $8 \mathrm{E}-08$ & E-08 & $3.11 \mathrm{E}-08$ & $3.10 \mathrm{E}-08$ & $3.12 \mathrm{E}-08$ \\
\hline Np-237 & & & $2.96 \mathrm{E}-08$ & $3.02 \mathrm{E}-08$ & $2 \mathrm{E}-08$ & & -08 & -08 & $E-08$ & -08 & $=-08$ & $=-08$ & $E-08$ & $2.96 \mathrm{E}-08$ & $2.96 \mathrm{E}-08$ \\
\hline Pu-238 & & 9.20 & 9.20 & -04 & -04 & & .04 & -04 & -04 & -04 & -04 & -04 & -04 & E-04 & E-04 \\
\hline Pu-239 & & & $2.65 \mathrm{E}-05$ & & & & & & -05 & & & -05 & $=-05$ & $5-05$ & $E-05$ \\
\hline Pu-240 & $9.40 \mathrm{E}-06$ & $9.39 \mathrm{E}-06$ & $9.39 \mathrm{E}-06$ & $6.69 \mathrm{E}-06$ & $6.69 \mathrm{E}-06$ & $8.63 \mathrm{E}-06$ & E-06 & E-06 & $6.69 \mathrm{E}-06$ & $6.86 \mathrm{E}-06$ & $6.86 \mathrm{E}-06$ & $9.38 \mathrm{E}-06$ & $9.39 \mathrm{E}-06$ & $9.38 \mathrm{E}-06$ & $2 \mathrm{E}-06$ \\
\hline Pu-241 & $5.28 \mathrm{E}-04$ & $5.28 \mathrm{E}-04$ & $5.28 \mathrm{E}-04$ & $4.56 \mathrm{E}-04$ & $4.56 \mathrm{E}-04$ & $5.06 \mathrm{E}-04$ & SE-04 & E-04 & $4.56 \mathrm{E}-04$ & $4.71 \mathrm{E}-04$ & $4.71 \mathrm{E}-04$ & $5.28 \mathrm{E}-04$ & $5.28 \mathrm{E}-04$ & $5.28 \mathrm{E}-04$ & $5.29 \mathrm{E}-04$ \\
\hline Pu-242 & $1.36 \mathrm{E}-08$ & $1.36 \mathrm{E}-08$ & $1.36 \mathrm{E}-08$ & $1.30 \mathrm{E}-08$ & $1.30 \mathrm{E}-08$ & 1.34E-08 & $1.33 \mathrm{E}-08$ & $1.30 \mathrm{E}-08$ & $1.30 \mathrm{E}-08$ & 1.67E-08 & $1.66 \mathrm{E}-08$ & $1.36 \mathrm{E}-08$ & $1.36 \mathrm{E}-08$ & $1.36 \mathrm{E}-08$ & $1.36 \mathrm{E}-08$ \\
\hline Am-241 & $9.42 \mathrm{E}-05$ & $9.42 \mathrm{E}-05$ & $9.42 \mathrm{E}-05$ & $9.48 \mathrm{E}-05$ & $9.48 \mathrm{E}-05$ & $1.86 \mathrm{E}-04$ & 1.87E-04 & $9.48 \mathrm{E}-05$ & $9.48 \mathrm{E}-05$ & 9.47E-05 & 9.47E-05 & $9.42 \mathrm{E}-05$ & $9.42 \mathrm{E}-05$ & $9.42 \mathrm{E}-05$ & $9.42 \mathrm{E}-05$ \\
\hline Am-242m & $8.85 \mathrm{E}-08$ & $8.85 \mathrm{E}-08$ & $8.85 \mathrm{E}-08$ & $8.93 \mathrm{E}-08$ & $8.93 \mathrm{E}-08$ & $2.14 \mathrm{E}-07$ & $2.15 \mathrm{E}-07$ & $8.93 \mathrm{E}-08$ & $8.93 \mathrm{E}-08$ & 8.92E-08 & 8.92E-08 & $8.85 \mathrm{E}-08$ & $8.85 \mathrm{E}-08$ & $8.85 \mathrm{E}-08$ & $8.85 \mathrm{E}-08$ \\
\hline Cm-242 & & -- & -- & -- & --- & --- & --- & --- & --- & --- & -- & -- & -- & -- & - \\
\hline Cm-244 & $9.68 \mathrm{E}-05$ & $9.68 \mathrm{E}-05$ & $9.68 \mathrm{E}-05$ & $9.68 \mathrm{E}-05$ & $9.68 \mathrm{E}-05$ & $9.69 \mathrm{E}-05$ & E-05 & $E-05$ & $9.68 \mathrm{E}-05$ & $9.68 \mathrm{E}-05$ & $9.68 \mathrm{E}-05$ & -05 & -05 & $9.68 \mathrm{E}-05$ & $9.68 \mathrm{E}-05$ \\
\hline Cm-245 & & & & & & & & & & & & & E-09 & $7.21 \mathrm{E}-09$ & \\
\hline Total Alpha & $1.15 \mathrm{E}-03$ & $1.15 \mathrm{E}-03$ & $1.15 \mathrm{E}-03$ & $1.05 \mathrm{E}-03$ & $1.05 \mathrm{E}-03$ & $1.15 \mathrm{E}-03$ & $E-03$ & $1.05 \mathrm{E}-03$ & $1.05 \mathrm{E}-03$ & $\overline{1.12}$ & $\overline{1.1}$ & E-03 & $1.15 \mathrm{E}-03$ & $1.15 \mathrm{E}-03$ & $1.15 \mathrm{E}-03$ \\
\hline
\end{tabular}


Table 10: Projected Feed Composition After Dissolution and Dilution to 6.44 M Total Sodium Concentration $100 \mathrm{mg} / \mathrm{L}$ sludge; $20 \%$ of interstitial supernate remaining

\begin{tabular}{|c|c|c|c|c|c|c|c|c|c|c|c|c|c|c|c|}
\hline \multicolumn{16}{|c|}{ Concentration (M) } \\
\hline & Tank 25 & Tank 27 & Tank 28 & Tank 29 & Tank 31 & Tank 33 & Tank 34 & Tank 36 & Tank 37 & Tank 38 & Tank 41 & Tank 44 & Tank 45 & Tank 46 & Tank 47 \\
\hline $\mathrm{Na}$ & $6.44 \mathrm{E}+00$ & $6.44 \mathrm{E}+00$ & $6.44 \mathrm{E}+00$ & $6.44 \mathrm{E}+00$ & $6.44 \mathrm{E}+00$ & $6.44 \mathrm{E}+00$ & $6.44 \mathrm{E}+00$ & $6.44 \mathrm{E}+00$ & $6.44 \mathrm{E}+00$ & $6.44 \mathrm{E}+00$ & $6.44 \mathrm{E}+00$ & $6.44 \mathrm{E}+00$ & $6.44 \mathrm{E}+00$ & $6.44 \mathrm{E}+00$ & $6.44 \mathrm{E}+00$ \\
\hline $\mathrm{Al}(\mathrm{OH}) 4$ & $3.21 \mathrm{E}-01$ & $3.29 \mathrm{E}-01$ & $3.21 \mathrm{E}-01$ & $3.99 \mathrm{E}-01$ & $4.00 \mathrm{E}-01$ & $3.20 \mathrm{E}-01$ & $3.19 \mathrm{E}-01$ & $3.89 \mathrm{E}-01$ & $3.95 \mathrm{E}-01$ & $4.00 \mathrm{E}-01$ & $3.94 \mathrm{E}-01$ & $3.18 \mathrm{E}-01$ & $3.16 \mathrm{E}-01$ & $3.12 \mathrm{E}-01$ & $3.22 \mathrm{E}-01$ \\
\hline $\mathrm{CO} 3$ & $2.20 \mathrm{E}-01$ & $2.19 \mathrm{E}-01$ & $2.18 \mathrm{E}-01$ & $1.33 \mathrm{E}-01$ & $1.31 \mathrm{E}-01$ & $2.23 \mathrm{E}-01$ & $2.26 \mathrm{E}-01$ & $1.31 \mathrm{E}-01$ & $1.31 \mathrm{E}-01$ & $1.35 \mathrm{E}-01$ & $1.34 \mathrm{E}-01$ & $2.20 \mathrm{E}-01$ & $2.22 \mathrm{E}-01$ & $2.17 \mathrm{E}-01$ & $2.24 \mathrm{E}-01$ \\
\hline $\mathbf{C 2 O 4}$ & $2.21 \mathrm{E}-02$ & $2.20 \mathrm{E}-02$ & $2.20 \mathrm{E}-02$ & $1.41 \mathrm{E}-02$ & $1.39 \mathrm{E}-02$ & $2.25 \mathrm{E}-02$ & $2.21 \mathrm{E}-02$ & $1.39 \mathrm{E}-02$ & $1.40 \mathrm{E}-02$ & $1.42 \mathrm{E}-02$ & $1.40 \mathrm{E}-02$ & $2.19 \mathrm{E}-02$ & $2.20 \mathrm{E}-02$ & $2.19 \mathrm{E}-02$ & $2.22 \mathrm{E}-02$ \\
\hline $\mathrm{NO2}$ & $1.19 \mathrm{E}-01$ & $1.20 \mathrm{E}-01$ & $1.24 \mathrm{E}-01$ & $7.46 \mathrm{E}-01$ & $7.56 \mathrm{E}-01$ & 1.02E-01 & 1.17E-01 & $7.52 \mathrm{E}-01$ & $7.48 \mathrm{E}-01$ & $7.44 \mathrm{E}-01$ & $7.40 \mathrm{E}-01$ & $1.21 \mathrm{E}-01$ & $1.24 \mathrm{E}-01$ & $1.22 \mathrm{E}-01$ & $1.09 \mathrm{E}-01$ \\
\hline $\mathrm{NO3}$ & $3.76 \mathrm{E}+00$ & $3.75 \mathrm{E}+00$ & $3.76 \mathrm{E}+00$ & $2.65 \mathrm{E}+00$ & $2.63 \mathrm{E}+00$ & $3.83 \mathrm{E}+00$ & & $2.62 \mathrm{E}+00$ & $2.63 \mathrm{E}+00$ & $2.67 \mathrm{E}+00$ & $2.66 \mathrm{E}+00$ & $3.74 \mathrm{E}+00$ & $3.74 \mathrm{E}+00$ & $3.74 \mathrm{E}+00$ & $3.80 \mathrm{E}+00$ \\
\hline $\mathrm{OH}$ & $1.02 \mathrm{E}+00$ & $1.02 \mathrm{E}+00$ & $9.85 \mathrm{E}-01$ & $1.85 \mathrm{E}+00$ & $1.87 \mathrm{E}+00$ & $9.20 \mathrm{E}-01$ & $9.75 \mathrm{E}-01$ & $1.94 \mathrm{E}+00$ & $1.89 \mathrm{E}+00$ & $1.86 \mathrm{E}+00$ & $1.86 \mathrm{E}+00$ & $1.05 \mathrm{E}+00$ & $1.07 \mathrm{E}+00$ & $9.69 \mathrm{E}-01$ & $9.45 \mathrm{E}-01$ \\
\hline PO4 & $1.53 \mathrm{E}-03$ & $1.53 \mathrm{E}-03$ & $1.62 \mathrm{E}-03$ & 1.57E-02 & $1.56 \mathrm{E}-02$ & $1.37 \mathrm{E}-03$ & $1.37 \mathrm{E}-03$ & 1.57E-02 & 1.55E-02 & 1.57E-02 & 1.57E-02 & $1.44 \mathrm{E}-03$ & 1.45E-03 & $1.51 \mathrm{E}-03$ & $1.37 \mathrm{E}-03$ \\
\hline SO4 & $3.21 \mathrm{E}-01$ & $3.20 \mathrm{E}-01$ & $3.21 \mathrm{E}-01$ & $33 \mathrm{E}-01$ & $1.80 \mathrm{E}-01$ & $3.28 \mathrm{E}-01$ & $2 \mathrm{E}-01$ & $1.80 \mathrm{E}-01$ & $1.80 \mathrm{E}-01$ & $1.85 \mathrm{E}-01$ & $1.82 \mathrm{E}-01$ & $3.19 \mathrm{E}-01$ & $3.20 \mathrm{E}-01$ & $3.19 \mathrm{E}-01$ & $3.24 \mathrm{E}-01$ \\
\hline $\mathbf{A g}$ & $1.21 \mathrm{E}-08$ & $7.10 \mathrm{E}-08$ & $\begin{array}{l}5.23 \mathrm{E}-08 \\
\end{array}$ & $2.65 \mathrm{E}-08$ & $2.26 \mathrm{E}-08$ & $2.49 \mathrm{E}-08$ & $2.45 \mathrm{E}-08$ & $2.26 \mathrm{E}-08$ & $2.26 \mathrm{E}-08$ & $2.92 \mathrm{E}-08$ & $2.27 \mathrm{E}-08$ & $2.43 \mathrm{E}-08$ & $2.44 \mathrm{E}-08$ & $2.43 \mathrm{E}-08$ & $2.46 \mathrm{E}-08$ \\
\hline As & $2.13 \mathrm{E}-06$ & $1.93 \mathrm{E}-06$ & $2.51 \mathrm{E}-06$ & $5 \mathrm{E}-06$ & $3.55 \mathrm{E}-05$ & $1.72 \mathrm{E}-05$ & $7 \mathrm{E}-05$ & $7 \mathrm{E}-05$ & $4.44 \mathrm{E}-05$ & $2.38 \mathrm{E}-06$ & $2.94 \mathrm{E}-05$ & $7.38 \mathrm{E}-05$ & $8.09 \mathrm{E}-05$ & $4.45 \mathrm{E}-05$ & $3.08 \mathrm{E}-05$ \\
\hline $\mathrm{Ba}$ & $2.11 \mathrm{E}-09$ & $2.11 \mathrm{E}-08$ & $1.58 \mathrm{E}-08$ & $1.39 \mathrm{E}-07$ & $-\infty$ & --- & $\cdots$ & ---- & & $4.99 \mathrm{E}-09$ & & $\mid---$ & & & \\
\hline $\mathrm{Ca}$ & $4.98 \mathrm{E}-05$ & $4.97 \mathrm{E}-05$ & $4.98 \mathrm{E}-05$ & $3.17 \mathrm{E}-05$ & $3.05 \mathrm{E}-05$ & $5.19 \mathrm{E}-05$ & $4.99 \mathrm{E}-05$ & 3.05E-05 & E-05 & $3.20 \mathrm{E}-05$ & $3.06 \mathrm{E}-01$ & E-05 & $4.97 \mathrm{E}-05$ & $4.95 \mathrm{E}-05$ & $5.04 \mathrm{E}-05$ \\
\hline $\mathbf{C d}$ & $5.80 \mathrm{E}-08$ & $5.53 \mathrm{E}-08$ & $7.34 \mathrm{E}-08$ & $8 \mathrm{E}-08$ & $9.57 \mathrm{E}-07$ & $2.06 \mathrm{E}-07$ & $\mathrm{E}-06$ & $E-06$ & $1.30 \mathrm{E}-06$ & $8 \mathrm{E}-08$ & $\mathrm{E}-07$ & $2.40 \mathrm{E}-06$ & $2.68 \mathrm{E}-06$ & $7 \mathrm{E}-06$ & $7.37 \mathrm{E}-07$ \\
\hline $\mathrm{Cl}$ & $4.23 \mathrm{E}-02$ & $4.22 \mathrm{E}-02$ & $4.23 \mathrm{E}-02$ & tE-02 & $9 \mathrm{E}-02$ & $4.29 \mathrm{E}-02$ & $\mathrm{E}-02$ & $\mathrm{E}-02$ & $3.79 \mathrm{E}-02$ & 3.87E-02 & $3.81 \mathrm{E}-02$ & $1 \mathrm{E}-02$ & $4.23 \mathrm{E}-02$ & $0 \mathrm{E}-02$ & $4.24 \mathrm{E}-02$ \\
\hline Co & 3.09E-07 & $3.15 \mathrm{E}-07$ & $2.60 \mathrm{E}-07$ & FE-07 & $2.02 \mathrm{E}-07$ & $1.32 \mathrm{E}-07$ & E-07 & $\mathrm{E}-07$ & $2.39 \mathrm{E}-07$ & $1.33 \mathrm{E}-07$ & $1.77 \mathrm{E}-07$ & $3.65 \mathrm{E}-07$ & $3.95 \mathrm{E}-07$ & $44 \mathrm{E}-07$ & $1.88 \mathrm{E}-07$ \\
\hline $\mathrm{Cr}$ & $1.28 \mathrm{E}-05$ & $7.79 \mathrm{E}-06$ & $2.59 \mathrm{E}-05$ & $\mathrm{E}-04$ & $\mathrm{E}-04$ & $75 \mathrm{E}-04$ & & & E-04 & E-04 & $\mathrm{E}-04$ & $\mathrm{E}-04$ & $\mathrm{E}-04$ & $8 \mathrm{E}-04$ & $1.61 \mathrm{E}-04$ \\
\hline $\mathrm{Cu}$ & -04 & E-04 & -05 & -05 & $E-05$ & E-05 & -05 & $=-04$ & $\mathrm{E}-05$ & E-05 & $5 \mathrm{E}-05$ & $\mathrm{E}-04$ & $9 \mathrm{E}-04$ & $4 \mathrm{E}-05$ & $4.38 \mathrm{E}-05$ \\
\hline $\mathrm{Fe}$ & $9.34 \mathrm{E}-07$ & $9.32 \mathrm{E}-07$ & $7.26 \mathrm{E}-07$ & $5.60 \mathrm{E}-07$ & $2.58 \mathrm{E}-06$ & $6.30 \mathrm{E}-07$ & $2 \mathrm{E}-06$ & $5.23 \mathrm{E}-06$ & BE-06 & $1.30 \mathrm{E}-06$ & $1.96 \mathrm{E}-06$ & 6.37E-06 & $7.08 \mathrm{E}-06$ & $3.41 \mathrm{E}-06$ & $2.02 \mathrm{E}-06$ \\
\hline$F$ & $7.04 \mathrm{E}-02$ & $02 \mathrm{E}-02$ & $E-02$ & $\mathrm{E}-02$ & E-02 & $\mathrm{E}-02$ & $\mathrm{E}-02$ & E-02 & $3.56 \mathrm{E}-02$ & $3.63 \mathrm{E}-02$ & $3.57 \mathrm{E}-02$ & $7.00 \mathrm{E}-02$ & $7.02 \mathrm{E}-02$ & $6.98 \mathrm{E}-02$ & $7.09 \mathrm{E}-02$ \\
\hline $\mathrm{Hg}$ & $7.23 \mathrm{E}-07$ & $7.21 \mathrm{E}-07$ & $7.22 \mathrm{E}-07$ & $1.36 \mathrm{E}-06$ & $8.20 \mathrm{E}-06$ & $8.61 \mathrm{E}-06$ & E-06 & $8.74 \mathrm{E}-06$ & $8.38 \mathrm{E}-06$ & $6.83 \mathrm{E}-07$ & $8.12 \mathrm{E}-06$ & 9.57E-06 & $9.73 \mathrm{E}-06$ & $8.95 \mathrm{E}-06$ & $8.79 \mathrm{E}-06$ \\
\hline $\mathrm{K}$ & $\begin{array}{l}6.61 \mathrm{E}-03 \\
\end{array}$ & $\begin{array}{l}6.74 \mathrm{E}-03 \\
\end{array}$ & 6.6 & $\mathrm{E}-03$ & $\mathrm{E}-03$ & $\mathrm{E}-03$ & -03 & $\begin{array}{l}6.64 \mathrm{E}-03 \\
\end{array}$ & $\mathrm{E}-03$ & $\mathrm{E}-03$ & $\mathrm{E}-03$ & $\begin{array}{l}7.01 \mathrm{E}-03 \\
\end{array}$ & $\begin{array}{l}7.31 \mathrm{E}-03 \\
\end{array}$ & $3 \mathrm{E}-03$ & $5.55 \mathrm{E}-03$ \\
\hline $\mathrm{Mg}$ & \begin{tabular}{l|l}
.-- \\
\end{tabular} & & $6.80 \mathrm{E}-09$ & $E-08$ & $9 \mathrm{E}-08$ & $1.08 \mathrm{E}-07$ & $2.19 \mathrm{E}-08$ & $\mid---$ & $6.92 \mathrm{E}-09$ & E-08 & $E-08$ & & & $1.78 \mathrm{E}-08$ & $6.32 \mathrm{E}-08$ \\
\hline$M n$ & $3.96 \mathrm{E}-08$ & $4.74 \mathrm{E}-08$ & $6.32 \mathrm{E}-08$ & $7.43 \mathrm{E}-08$ & $1.34 \mathrm{E}-07$ & $6.99 \mathrm{E}-08$ & $1.63 \mathrm{E}-07$ & $2.26 \mathrm{E}-07$ & $1.65 \mathrm{E}-07$ & $3.99 \mathrm{E}-08$ & $1.12 \mathrm{E}-07$ & $2.69 \mathrm{E}-07$ & $2.94 \mathrm{E}-07$ & $1.66 \mathrm{E}-07$ & $1.18 \mathrm{E}-07$ \\
\hline Nd & \begin{tabular}{|l|}
--- \\
\end{tabular} & --- & 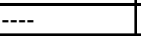 & & --- & $3.91 \mathrm{E}-06$ & --- & $\begin{array}{ll}---- \\
\end{array}$ & \begin{tabular}{|l|}
--- \\
\end{tabular} & & $\mathrm{E}-06$ & \begin{tabular}{|l|}
---- \\
\end{tabular} & --- & --- & $1.35 \mathrm{E}-06$ \\
\hline $\mathrm{Ni}$ & 7.40E-07 & 7.57E-07 & $6.09 \mathrm{E}-07$ & $2.88 \mathrm{E}-07$ & $4.63 \mathrm{E}-07$ & $2.68 \mathrm{E}-07$ & $5.59 \mathrm{E}-07$ & 7.52E-07 & 5.61E-07 & $2.76 \mathrm{E}-07$ & $3.95 \mathrm{E}-07$ & $8.91 \mathrm{E}-07$ & 9.69E-07 & 5.67E-07 & $4.17 \mathrm{E}-07$ \\
\hline $\mathrm{Pb}$ & $2.03 \mathrm{E}-06$ & $1.67 \mathrm{E}-06$ & $2.31 \mathrm{E}-06$ & $3.35 \mathrm{E}-06$ & 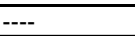 & ---- & $\mid---$ & ---- & ---- & EE-06 & 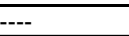 & $\mid---$ & $\mid---$ & \begin{tabular}{|l|}
--- \\
\end{tabular} & \\
\hline $\mathbf{R u}$ & $1.17 \mathrm{E}-05$ & $1.21 \mathrm{E}-05$ & 9.07E-06 & $2.78 \mathrm{E}-06$ & $6.36 \mathrm{E}-06$ & $2.10 \mathrm{E}-06$ & $4 \mathrm{E}-06$ & $1.22 \mathrm{E}-05$ & $8.34 \mathrm{E}-06$ & E-06 & $4.97 \mathrm{E}-06$ & $1.48 \mathrm{E}-05$ & \begin{tabular}{|l|l|}
$5.64 \mathrm{E}-05$ \\
\end{tabular} & $8.24 \mathrm{E}-06$ & $5.16 \mathrm{E}-06$ \\
\hline Se & $1.51 \mathrm{E}-05$ & $1.15 \mathrm{E}-05$ & $1.94 \mathrm{E}-05$ & $4.05 \mathrm{E}-05$ & $1.09 \mathrm{E}-04$ & $1.46 \mathrm{E}-04$ & $1.13 \mathrm{E}-04$ & $7.90 \mathrm{E}-05$ & $9.89 \mathrm{E}-05$ & E-05 & $1.17 \mathrm{E}-04$ & $7.68 \mathrm{E}-05$ & $6.90 \mathrm{E}-05$ & 1.10E-04 & $1.28 \mathrm{E}-04$ \\
\hline$\overline{S i}$ & $7.17 \mathrm{E}-05$ & $6.28 \mathrm{E}-05$ & $3.68 \mathrm{E}-04$ & $1.21 \mathrm{E}-05$ & $-x_{-1}$ & \begin{tabular}{|l|}
--- \\
\end{tabular} & ---- & ---- & ---- & $9.51 \mathrm{E}-05$ & --- & ---- & ---- & ---- & $-\cdots$ \\
\hline $\mathrm{Sr}$ & \begin{tabular}{|l|}
--- \\
\end{tabular} & \begin{tabular}{l|l}
--- & \\
-
\end{tabular} & --- & $1.96 \mathrm{E}-06$ & ---- & $2.71 \mathrm{E}-06$ & ---- & \begin{tabular}{|l|}
---- \\
\end{tabular} & ---- & $2.16 \mathrm{E}-06$ & $5.35 \mathrm{E}-07$ & ---- & $\mid---$ & $-{ }_{---}$ & $7.27 \mathrm{E}-07$ \\
\hline $\mathbf{Z n}$ & \begin{tabular}{|l|}
$1.35 \mathrm{E}-04$ \\
\end{tabular} & $1.39 \mathrm{E}-04$ & $1.05 \mathrm{E}-04$ & $3.51 \mathrm{E}-05$ & $7.48 \mathrm{E}-05$ & $2.79 \mathrm{E}-05$ & 9.39E-05 & $1.40 \mathrm{E}-04$ & 9.69E-05 & $3.20 \mathrm{E}-05$ & $5.94 \mathrm{E}-05$ & $1.69 \mathrm{E}-04$ & $1.86 \mathrm{E}-04$ & $9.61 \mathrm{E}-05$ & \\
\hline $\mathrm{Zr}$ & $3.55 \mathrm{E}-06$ & $3.66 \mathrm{E}-06$ & $2.70 \mathrm{E}-06$ & $7.09 \mathrm{E}-07$ & $1.85 \mathrm{E}-06$ & $4.76 \mathrm{E}-07$ & $2.37 \mathrm{E}-06$ & $3.72 \mathrm{E}-06$ & $2.48 \mathrm{E}-06$ & $6.19 \mathrm{E}-07$ & $1.41 \mathrm{E}-06$ & $4.52 \mathrm{E}-06$ & $5.03 \mathrm{E}-06$ & $2.44 \mathrm{E}-06$ & $1.46 \mathrm{E}-06$ \\
\hline Insol.Sol.mg/L & 100 & 100 & 100 & 100 & 100 & 100 & 100 & 100 & 100 & 100 & 100 & 100 & 100 & 100 & 100 \\
\hline
\end{tabular}


Table 10: Projected Feed Composition After Dissolution and Dilution to 6.44 M Total Sodium Concentration $100 \mathrm{mg} / \mathrm{L}$ sludge; $20 \%$ of interstitial supernate remaining (continued)

\begin{tabular}{|c|c|c|c|c|c|c|c|c|c|c|c|c|c|c|c|}
\hline \multicolumn{16}{|c|}{ Radionuclide Composition (Ci/gal) } \\
\hline & Tank 25 & Tank 27 & Tank 28 & Tank 29 & Tank 31 & Tank 33 & Tank 34 & Tank 36 & Tank 37 & Tank 38 & Tank 41 & Tank 44 & Tank 45 & Tank 46 & Tank 47 \\
\hline C-14 & $5.62 \mathrm{E}-10$ & $5.62 \mathrm{E}-10$ & $5.62 \mathrm{E}-10$ & $5.62 \mathrm{E}-10$ & $5.62 \mathrm{E}-10$ & $5.62 \mathrm{E}-10$ & $5.62 \mathrm{E}-10$ & $5.62 \mathrm{E}-10$ & $5.62 \mathrm{E}-10$ & $5.62 \mathrm{E}-10$ & $5.62 \mathrm{E}-10$ & $5.62 \mathrm{E}-10$ & $5.62 \mathrm{E}-10$ & $5.62 \mathrm{E}-10$ & $5.62 \mathrm{E}-10$ \\
\hline Ni-59 & $3.23 \mathrm{E}-07$ & $3.23 \mathrm{E}-07$ & $3.23 \mathrm{E}-07$ & $3.23 \mathrm{E}-07$ & $3.23 \mathrm{E}-07$ & $3.23 \mathrm{E}-07$ & $3.23 \mathrm{E}-07$ & $3.23 \mathrm{E}-07$ & $3.23 \mathrm{E}-07$ & $3.23 \mathrm{E}-07$ & $3.23 \mathrm{E}-07$ & $3.23 \mathrm{E}-07$ & $3.23 \mathrm{E}-07$ & $3.23 \mathrm{E}-07$ & $3.23 \mathrm{E}-07$ \\
\hline $\mathrm{Ni}-63$ & $5.02 \mathrm{E}-10$ & $5.02 \mathrm{E}-10$ & $5.02 \mathrm{E}-10$ & $5.02 \mathrm{E}-10$ & $5.02 \mathrm{E}-10$ & $5.02 \mathrm{E}-10$ & $5.02 \mathrm{E}-10$ & $5.02 \mathrm{E}-10$ & $5.02 \mathrm{E}-10$ & $5.02 \mathrm{E}-10$ & $5.02 \mathrm{E}-10$ & $5.02 \mathrm{E}-10$ & $5.02 \mathrm{E}-10$ & $5.02 \mathrm{E}-10$ & $5.02 \mathrm{E}-10$ \\
\hline Co-60 & $5.14 \mathrm{E}-05$ & $5.14 \mathrm{E}-05$ & $5.14 \mathrm{E}-05$ & $5.14 \mathrm{E}-05$ & $5.14 \mathrm{E}-05$ & $5.14 \mathrm{E}-05$ & $5.14 \mathrm{E}-05$ & $5.14 \mathrm{E}-05$ & $5.14 \mathrm{E}-05$ & $5.14 \mathrm{E}-05$ & $5.14 \mathrm{E}-05$ & $5.14 \mathrm{E}-05$ & $5.14 \mathrm{E}-05$ & $5.14 \mathrm{E}-05$ & $5.14 \mathrm{E}-05$ \\
\hline Se-79 & $1.91 \mathrm{E}-07$ & $1.91 \mathrm{E}-07$ & $1.91 \mathrm{E}-07$ & $1.91 \mathrm{E}-07$ & $1.91 \mathrm{E}-07$ & $1.91 \mathrm{E}-07$ & $1.91 \mathrm{E}-07$ & $1.91 \mathrm{E}-07$ & $1.91 \mathrm{E}-07$ & $1.91 \mathrm{E}-07$ & $1.91 \mathrm{E}-07$ & $1.91 \mathrm{E}-07$ & $1.91 \mathrm{E}-07$ & $1.91 \mathrm{E}-07$ & $1.91 \mathrm{E}-07$ \\
\hline \begin{tabular}{|l|} 
Tc-99 \\
\end{tabular} & $3.24 \mathrm{E}-06$ & $3.24 \mathrm{E}-06$ & $3.24 \mathrm{E}-06$ & $3.24 \mathrm{E}-06$ & $3.24 \mathrm{E}-06$ & $3.24 \mathrm{E}-06$ & $3.24 \mathrm{E}-06$ & $3.24 \mathrm{E}-06$ & $3.24 \mathrm{E}-06$ & $3.24 \mathrm{E}-06$ & $3.24 \mathrm{E}-06$ & $3.24 \mathrm{E}-06$ & $3.24 \mathrm{E}-06$ & $3.24 \mathrm{E}-06$ & $3.24 \mathrm{E}-06$ \\
\hline \begin{tabular}{|l|} 
Ru-106 \\
\end{tabular} & $1.20 \mathrm{E}-06$ & $1.20 \mathrm{E}-06$ & $1.20 \mathrm{E}-06$ & $1.20 \mathrm{E}-06$ & $1.20 \mathrm{E}-06$ & $1.20 \mathrm{E}-06$ & $1.20 \mathrm{E}-06$ & $1.20 \mathrm{E}-06$ & $1.20 \mathrm{E}-06$ & $1.20 \mathrm{E}-06$ & $1.20 \mathrm{E}-06$ & $1.20 \mathrm{E}-06$ & $1.20 \mathrm{E}-06$ & $1.20 \mathrm{E}-06$ & $1.20 \mathrm{E}-06$ \\
\hline \begin{tabular}{|l|} 
Rh-106 \\
\end{tabular} & $1.20 \mathrm{E}-06$ & $1.20 \mathrm{E}-06$ & $1.20 \mathrm{E}-06$ & $1.20 \mathrm{E}-06$ & $1.20 \mathrm{E}-06$ & $1.20 \mathrm{E}-06$ & $1.20 \mathrm{E}-06$ & $1.20 \mathrm{E}-06$ & $1.20 \mathrm{E}-06$ & $1.20 \mathrm{E}-06$ & $1.20 \mathrm{E}-06$ & $1.20 \mathrm{E}-06$ & $1.20 \mathrm{E}-06$ & $1.20 \mathrm{E}-06$ & $1.20 \mathrm{E}-06$ \\
\hline Sb-125 & $3.07 \mathrm{E}-05$ & $3.07 \mathrm{E}-05$ & $3.07 \mathrm{E}-05$ & $3.07 \mathrm{E}-05$ & $3.07 \mathrm{E}-05$ & $3.07 \mathrm{E}-05$ & $3.07 \mathrm{E}-05$ & $3.07 \mathrm{E}-05$ & $3.07 \mathrm{E}-05$ & $3.07 \mathrm{E}-05$ & $3.07 \mathrm{E}-05$ & $3.07 \mathrm{E}-05$ & $3.07 \mathrm{E}-05$ & $3.07 \mathrm{E}-05$ & $3.07 \mathrm{E}-05$ \\
\hline Sn-126 & $2.55 \mathrm{E}-07$ & $2.55 \mathrm{E}-07$ & $2.55 \mathrm{E}-07$ & $2.55 \mathrm{E}-07$ & $2.55 \mathrm{E}-07$ & $2.55 \mathrm{E}-07$ & $2.55 \mathrm{E}-07$ & $5 \mathrm{E}-07$ & $2.55 \mathrm{E}-07$ & $2.55 \mathrm{E}-07$ & $2.55 \mathrm{E}-07$ & $2.55 \mathrm{E}-07$ & $2.55 \mathrm{E}-07$ & $2.55 \mathrm{E}-07$ & $2.55 \mathrm{E}-07$ \\
\hline \begin{tabular}{|l|}
$1-129$ \\
\end{tabular} & $2.98 \mathrm{E}-11$ & $2.98 \mathrm{E}-11$ & $2.98 \mathrm{E}-11$ & $2.98 \mathrm{E}-11$ & $2.98 \mathrm{E}-11$ & $2.98 \mathrm{E}-11$ & & & $2.98 \mathrm{E}-11$ & $2.98 \mathrm{E}-11$ & $2.98 \mathrm{E}-11$ & $2.98 \mathrm{E}-11$ & $2.98 \mathrm{E}-11$ & $2.98 \mathrm{E}-11$ & \\
\hline \begin{tabular}{|l|} 
Cs-134 \\
\end{tabular} & $1.34 \mathrm{E}-06$ & $1.34 \mathrm{E}-06$ & $1.34 \mathrm{E}-06$ & $1.34 \mathrm{E}-06$ & $1.34 \mathrm{E}-06$ & $1.34 \mathrm{E}-06$ & $1.34 \mathrm{E}-06$ & $1.34 \mathrm{E}-06$ & $1.34 \mathrm{E}-06$ & $1.34 \mathrm{E}-06$ & $1.34 \mathrm{E}-06$ & $1.34 \mathrm{E}-06$ & $1.34 \mathrm{E}-06$ & $1.34 \mathrm{E}-06$ & $1.34 \mathrm{E}-06$ \\
\hline Cs-135 & $2.28 \mathrm{E}-09$ & $2.28 \mathrm{E}-09$ & $2.28 \mathrm{E}-09$ & $2.28 \mathrm{E}-09$ & $2.28 \mathrm{E}-09$ & $2.28 \mathrm{E}-09$ & $2.28 \mathrm{E}-09$ & $2.28 \mathrm{E}-09$ & $2.28 \mathrm{E}-09$ & $2.28 \mathrm{E}-09$ & $2.28 \mathrm{E}-09$ & $2.28 \mathrm{E}-09$ & $2.28 \mathrm{E}-09$ & $2.28 \mathrm{E}-09$ & $2.28 \mathrm{E}-09$ \\
\hline \begin{tabular}{|l|} 
Cs-137 \\
\end{tabular} & $6.29 \mathrm{E}-02$ & $5.96 \mathrm{E}-02$ & $6.54 \mathrm{E}-02$ & $5.63 \mathrm{E}-02$ & $1.53 \mathrm{E}-01$ & $2.15 \mathrm{E}-03$ & $5.12 \mathrm{E}-02$ & $\mathrm{E}-01$ & $2.04 \mathrm{E}-01$ & $4.38 \mathrm{E}-03$ & $3.46 \mathrm{E}-02$ & $7.71 \mathrm{E}-02$ & $7.31 \mathrm{E}-02$ & $9.38 \mathrm{E}-02$ & $3.96 \mathrm{E}-02$ \\
\hline Ba-137m & $5.95 \mathrm{E}-02$ & $5.64 \mathrm{E}-02$ & $6.19 \mathrm{E}-02$ & $5.33 \mathrm{E}-02$ & 1.44E-01 & $2.03 \mathrm{E}-03$ & $5 \mathrm{E}-02$ & $\mathrm{E}-01$ & $1.93 \mathrm{E}-01$ & $4.15 \mathrm{E}-03$ & $3.28 \mathrm{E}-02$ & $7.30 \mathrm{E}-02$ & $6.92 \mathrm{E}-02$ & $8.88 \mathrm{E}-02$ & $3.75 \mathrm{E}-02$ \\
\hline Sr-90 & $1.13 \mathrm{E}-02$ & $1.13 \mathrm{E}-02$ & $1.13 \mathrm{E}-02$ & $1.14 \mathrm{E}-02$ & $1.14 \mathrm{E}-02$ & $1.14 \mathrm{E}-02$ & & $\mathrm{E}-02$ & $1.14 \mathrm{E}-02$ & $1.13 \mathrm{E}-02$ & $1.13 \mathrm{E}-02$ & $1.13 \mathrm{E}-02$ & $1.13 \mathrm{E}-02$ & $1.13 \mathrm{E}-02$ & $1.13 \mathrm{E}-02$ \\
\hline Y-90 & $1.13 \mathrm{E}-02$ & $1.13 \mathrm{E}-02$ & $1.13 \mathrm{E}-02$ & $1.14 \mathrm{E}-02$ & $1.14 \mathrm{E}-02$ & $1.14 \mathrm{E}-02$ & $1.14 \mathrm{E}-02$ & $1.14 \mathrm{E}-02$ & $1.14 \mathrm{E}-02$ & $\begin{array}{l}1.13 \mathrm{E}-02 \\
\end{array}$ & $1.13 \mathrm{E}-02$ & $1.13 \mathrm{E}-02$ & $1.13 \mathrm{E}-02$ & $1.13 \mathrm{E}-02$ & $1.13 \mathrm{E}-02$ \\
\hline $\mathrm{H}-3$ & ---- & ---- & ---- & ---- & ---- & ---- & --- & ---- & ---- & $\mid---$ & $\mid---$ & ---- & ---- & ---- & \\
\hline \begin{tabular}{|l|} 
Ce-144 \\
\end{tabular} & $6.17 \mathrm{E}-07$ & $6.17 \mathrm{E}-07$ & 6.17E-07 & \begin{tabular}{|c|}
$6.17 \mathrm{E}-07$ \\
\end{tabular} & 6.17E-07 & $6.17 \mathrm{E}-07$ & $6.17 \mathrm{E}-07$ & 6.17E-07 & 6.17E-07 & \begin{tabular}{|c|}
$6.17 \mathrm{E}-07$ \\
\end{tabular} & $6.17 \mathrm{E}-07$ & \begin{tabular}{l|l|}
7 & $6.17 \mathrm{E}-07$ \\
\end{tabular} & \begin{tabular}{|c|}
$6.17 \mathrm{E}-07$ \\
\end{tabular} & 6.17E-07 & $6.17 \mathrm{E}-07$ \\
\hline \begin{tabular}{|l|} 
Pr-144 \\
\end{tabular} & $6.17 \mathrm{E}-07$ & $6.17 \mathrm{E}-07$ & $6.17 \mathrm{E}-07$ & $6.17 \mathrm{E}-07$ & $6.17 \mathrm{E}-07$ & $6.17 \mathrm{E}-07$ & E-07 & E-07 & $6.17 \mathrm{E}-07$ & $6.17 \mathrm{E}-07$ & $6.17 \mathrm{E}-07$ & $7 \mathrm{E}-07$ & $6.17 \mathrm{E}-07$ & $7 \mathrm{E}-07$ & $6.17 \mathrm{E}-07$ \\
\hline $\begin{array}{l}\text { Pm-147 } \\
\end{array}$ & $6.46 \mathrm{E}-04$ & $6.46 \mathrm{E}-04$ & $6.46 \mathrm{E}-04$ & $6.46 \mathrm{E}-04$ & $6.46 \mathrm{E}-04$ & $6.46 \mathrm{E}-04$ & $\mathrm{E}-04$ & E-04 & E-04 & $6 \mathrm{E}-04$ & $6.46 \mathrm{E}-04$ & $6.46 \mathrm{E}-04$ & $6.46 \mathrm{E}-04$ & $6 \mathrm{E}-04$ & $6.46 \mathrm{E}-04$ \\
\hline Eu-154 & $1.26 \mathrm{E}-04$ & $26 \mathrm{E}-04$ & $26 \mathrm{E}-04$ & $26 \mathrm{E}-04$ & $1.26 \mathrm{E}-04$ & $1.26 \mathrm{E}-04$ & -04 & $=-04$ & E-04 & E-04 & $1.26 \mathrm{E}-04$ & $6 \mathrm{E}-04$ & $1.26 \mathrm{E}-04$ & $26 \mathrm{E}-04$ & $1.26 \mathrm{E}-04$ \\
\hline Th-232 & $3.62 \mathrm{E}-10$ & $62 \mathrm{E}-10$ & $62 \mathrm{E}-10$ & $69 \mathrm{E}-10$ & $3.69 \mathrm{E}-10$ & $3.62 \mathrm{E}-10$ & $\mathrm{E}-10$ & $\mathrm{E}-10$ & $69 \mathrm{E}-10$ & $2 \mathrm{E}-10$ & $3.62 \mathrm{E}-10$ & $3.62 \mathrm{E}-10$ & $3.62 \mathrm{E}-10$ & $2 \mathrm{E}-10$ & $3.62 \mathrm{E}-10$ \\
\hline U-232 & $1.29 \mathrm{E}-10$ & $28 \mathrm{E}-10$ & $28 \mathrm{E}-10$ & $01 \mathrm{E}-11$ & $01 \mathrm{E}-11$ & $7.57 \mathrm{E}-10$ & $\mathrm{E}-10$ & $\mathrm{E}-11$ & E-11 & $7.80 \mathrm{E}-11$ & $7.80 \mathrm{E}-11$ & $1.28 \mathrm{E}-10$ & $1.28 \mathrm{E}-10$ & $1.28 \mathrm{E}-10$ & $1.29 \mathrm{E}-10$ \\
\hline \begin{tabular}{|l|}
$U-233$ \\
\end{tabular} & $1.22 \mathrm{E}-08$ & $22 \mathrm{E}-08$ & $22 \mathrm{E}-08$ & $37 \mathrm{E}-08$ & $37 \mathrm{E}-08$ & $1.22 \mathrm{E}-08$ & $\mathrm{E}-08$ & -08 & E-08 & $2 \mathrm{E}-08$ & $1.22 \mathrm{E}-08$ & $1.22 \mathrm{E}-08$ & $1.22 \mathrm{E}-08$ & $.22 \mathrm{E}-08$ & $1.22 \mathrm{E}-08$ \\
\hline \begin{tabular}{|l|} 
\\
\end{tabular}$-234$ & $3.92 \mathrm{E}-09$ & $3.92 \mathrm{E}-09$ & $3.92 \mathrm{E}-09$ & $13 \mathrm{E}-09$ & $4.13 \mathrm{E}-09$ & $3.92 \mathrm{E}-09$ & E-09 & E-09 & $3 \mathrm{E}-09$ & $7.40 \mathrm{E}-09$ & $7.34 \mathrm{E}-09$ & $3.92 \mathrm{E}-09$ & $3.92 \mathrm{E}-09$ & $3.92 \mathrm{E}-09$ & $3.92 \mathrm{E}-09$ \\
\hline U-235 & E-10 & $06 \mathrm{E}-10$ & $06 \mathrm{E}-10$ & E-10 & E-10 & $9.98 \mathrm{E}-10$ & & & E-10 & E-10 & $2.28 \mathrm{E}-10$ & E-10 & $3.06 \mathrm{E}-10$ & E-10 & $07 \mathrm{E}-10$ \\
\hline \begin{tabular}{|l|}
$U-236$ \\
\end{tabular} & $56 \mathrm{E}-10$ & $56 \mathrm{E}-10$ & $56 \mathrm{E}-10$ & $78 \mathrm{E}-10$ & $77 \mathrm{E}-10$ & $5.56 \mathrm{E}-10$ & $\mathrm{E}-10$ & -10 & $\mathrm{E}-10$ & $9 \mathrm{E}-09$ & $1.08 \mathrm{E}-09$ & $6 \mathrm{E}-10$ & $5.56 \mathrm{E}-10$ & $6 \mathrm{E}-10$ & $5.56 \mathrm{E}-10$ \\
\hline \begin{tabular}{|l|}
$U-238$ \\
\end{tabular} & $1.63 \mathrm{E}-08$ & $1.62 \mathrm{E}-08$ & $1.62 \mathrm{E}-08$ & $6.00 \mathrm{E}-09$ & $6.00 \mathrm{E}-09$ & $7.94 \mathrm{E}-08$ & $\mathrm{E}-08$ & -09 & E-09 & $4 \mathrm{E}-09$ & $5.94 \mathrm{E}-09$ & $1.62 \mathrm{E}-08$ & $1.62 \mathrm{E}-08$ & $1.62 \mathrm{E}-08$ & $1.63 \mathrm{E}-08$ \\
\hline \begin{tabular}{|l} 
Np-237 \\
\end{tabular} & & & & & & & & & & & & & & $7 \mathrm{E}-09$ & $8.47 \mathrm{E}-09$ \\
\hline \begin{tabular}{|l|} 
Pu-238 \\
\end{tabular} & $3.24 \mathrm{E}-04$ & $24 \mathrm{E}-04$ & $3.24 \mathrm{E}-04$ & $2.41 \mathrm{E}-04$ & $2.41 \mathrm{E}-04$ & $2.38 \mathrm{E}-04$ & $8 \mathrm{E}-04$ & E-04 & $2.41 \mathrm{E}-04$ & $3.34 \mathrm{E}-04$ & $3.33 \mathrm{E}-04$ & $3.24 \mathrm{E}-04$ & $3.24 \mathrm{E}-04$ & $3.23 \mathrm{E}-04$ & $3.25 \mathrm{E}-04$ \\
\hline \begin{tabular}{|l} 
Pu-239 \\
\end{tabular} & $1.63 \mathrm{E}-05$ & E-05 & $63 \mathrm{E}-05$ & E-06 & $4.15 \mathrm{E}-06$ & $1.29 \mathrm{E}-05$ & & & & E-06 & E-06 & BE-05 & $1.63 \mathrm{E}-05$ & $2 \mathrm{E}-05$ & $64 \mathrm{E}-05$ \\
\hline Pu-240 & $3 \mathrm{E}-06$ & E-06 & & E-06 & $1.93 \mathrm{E}-06$ & $3.87 \mathrm{E}-06$ & & & & & E-06 & E-06 & $3 \mathrm{E}-06$ & $\frac{5-06}{1 \mathrm{E}-06}$ & $4.65 \mathrm{E}-06$ \\
\hline Pu-241 & $2.03 \mathrm{E}-04$ & $03 \mathrm{E}-04$ & $2.03 \mathrm{E}-04$ & $1.31 \mathrm{E}-04$ & $1.31 \mathrm{E}-04$ & $1.81 \mathrm{E}-04$ & E-04 & -04 & E-04 & $6 \mathrm{E}-04$ & $1.46 \mathrm{E}-04$ & $2.03 \mathrm{E}-04$ & $2.03 \mathrm{E}-04$ & $2.03 \mathrm{E}-04$ & $2.04 \mathrm{E}-04$ \\
\hline \begin{tabular}{|l} 
Pu-242 \\
\end{tabular} & & & $4.28 \mathrm{E}-09$ & $3.73 \mathrm{E}-09$ & & & & & & & $7.32 \mathrm{E}-09$ & & $4.28 \mathrm{E}-09$ & $4.28 \mathrm{E}-09$ & $4.28 \mathrm{E}-09$ \\
\hline Am-241 & $2.69 \mathrm{E}-05$ & $2.69 \mathrm{E}-05$ & $2.69 \mathrm{E}-05$ & $2.75 \mathrm{E}-05$ & $2.75 \mathrm{E}-05$ & $1.19 \mathrm{E}-04$ & $1.20 \mathrm{E}-04$ & $2.75 \mathrm{E}-05$ & $2.75 \mathrm{E}-05$ & $2.74 \mathrm{E}-05$ & $2.74 \mathrm{E}-05$ & \begin{tabular}{|l|l|l} 
\\
$2.69 \mathrm{E}-05$
\end{tabular} & \begin{tabular}{|l|l|}
$2.60 \mathrm{E}-05$ \\
\end{tabular} & $2.69 \mathrm{E}-05$ & $\begin{array}{l}2.20 \mathrm{~L}-05 \\
2.69 \mathrm{E}-05\end{array}$ \\
\hline$A m-242 m$ & $2.53 \mathrm{E}-08$ & $2.53 \mathrm{E}-08$ & $2.53 \mathrm{E}-08$ & $2.60 \mathrm{E}-08$ & $2.60 \mathrm{E}-08$ & $1.51 \mathrm{E}-07$ & $1.51 \mathrm{E}-07$ & $2.60 \mathrm{E}-08$ & $2.60 \mathrm{E}-08$ & $2.59 \mathrm{E}-08$ & $2.59 \mathrm{E}-08$ & $2.53 \mathrm{E}-08$ & $2.53 \mathrm{E}-08$ & $2.53 \mathrm{E}-08$ & $2.53 \mathrm{E}-08$ \\
\hline \begin{tabular}{|l|}
$\mathrm{Cm}-242$ \\
\end{tabular} & ---- & - & ---- & ---- & ---- & ---- & ---- & ---- & ---- & \begin{tabular}{|l|}
---- \\
\end{tabular} & ---- & $\mid---$ & ---- & $\mid---$ & ---- \\
\hline Cm-244 & $2.77 \mathrm{E}-05$ & $2.77 \mathrm{E}-05$ & $2.77 \mathrm{E}-05$ & $2.77 \mathrm{E}-05$ & $2.77 \mathrm{E}-05$ & $2.77 \mathrm{E}-05$ & $2.77 \mathrm{E}-05$ & $7 \mathrm{E}-05$ & 2.77E-05 & $2.77 \mathrm{E}-05$ & $2.77 \mathrm{E}-05$ & $2.77 \mathrm{E}-05$ & $2.77 \mathrm{E}-05$ & $2.77 \mathrm{E}-05$ & $2.77 \mathrm{E}-05$ \\
\hline Cm-245 & $2.06 \mathrm{E}-09$ & $2.06 \mathrm{E}-09$ & 2.06E-09 & $2.06 \mathrm{E}-09$ & $2.06 \mathrm{E}-09$ & 2.06E-09 & $6 \mathrm{E}-09$ & E-09 & $2.06 \mathrm{E}-09$ & $2.06 \mathrm{E}-09$ & $2.06 \mathrm{E}-09$ & $2.06 \mathrm{E}-09$ & $2.06 \mathrm{E}-09$ & $2.06 \mathrm{E}-09$ & $2.06 \mathrm{E}-09$ \\
\hline Total Alpha & $4.00 \mathrm{E}-04$ & $3.99 \mathrm{E}-04$ & $3.99 \mathrm{E}-04$ & $3.02 \mathrm{E}-04$ & $3.02 \mathrm{E}-04$ & $4.02 \mathrm{E}-04$ & $4.01 \mathrm{E}-04$ & $3.02 \mathrm{E}-04$ & $3.02 \mathrm{E}-04$ & $3.96 \mathrm{E}-04$ & $3.94 \mathrm{E}-04$ & $3.99 \mathrm{E}-04$ & $3.99 \mathrm{E}-04$ & $3.99 \mathrm{E}-04$ & $4.00 \mathrm{E}-04$ \\
\hline
\end{tabular}


Table 11: Projected Feed Composition After Dissolution and Dilution to 6.44 M Total Sodium Concentration no sludge; $20 \%$ of interstitial supernate remaining

\begin{tabular}{|c|c|c|c|c|c|c|c|c|c|c|c|c|c|c|c|}
\hline \multicolumn{16}{|c|}{ Concentration (M) } \\
\hline & Tank 25 & Tank 27 & Tank 28 & Tank 29 & Tank 31 & Tank 33 & Tank 34 & Tank 36 & Tank 37 & Tank 38 & Tank 41 & Tank 44 & Tank 45 & Tank 46 & Tank 47 \\
\hline $\mathrm{Na}$ & $6.44 \mathrm{E}+00$ & $6.44 \mathrm{E}+00$ & $6.44 \mathrm{E}+00$ & $6.44 \mathrm{E}+00$ & $6.44 \mathrm{E}+00$ & $6.44 \mathrm{E}+00$ & $6.44 \mathrm{E}+00$ & $6.44 \mathrm{E}+00$ & $6.44 \mathrm{E}+00$ & $6.44 \mathrm{E}+00$ & $6.44 \mathrm{E}+00$ & $6.44 \mathrm{E}+00$ & $6.44 \mathrm{E}+00$ & $6.44 \mathrm{E}+00$ & $6.44 \mathrm{E}+00$ \\
\hline $\mathrm{Al}(\mathrm{OH}) 4$ & $3.21 \mathrm{E}-01$ & $3.29 \mathrm{E}-01$ & $3.21 \mathrm{E}-01$ & $3.99 \mathrm{E}-01$ & $4.00 \mathrm{E}-01$ & $3.20 \mathrm{E}-01$ & $3.19 \mathrm{E}-01$ & $3.89 \mathrm{E}-01$ & $3.95 \mathrm{E}-01$ & $4.00 \mathrm{E}-01$ & $3.94 \mathrm{E}-01$ & $3.18 \mathrm{E}-01$ & $3.16 \mathrm{E}-01$ & $3.12 \mathrm{E}-01$ & $3.22 \mathrm{E}-01$ \\
\hline $\mathrm{CO} 3$ & $2.20 \mathrm{E}-01$ & $2.19 \mathrm{E}-01$ & $2.18 \mathrm{E}-01$ & $1.33 \mathrm{E}-01$ & $1.31 \mathrm{E}-01$ & $2.23 \mathrm{E}-01$ & $2.26 \mathrm{E}-01$ & $1.31 \mathrm{E}-01$ & $1.31 \mathrm{E}-01$ & $1.35 \mathrm{E}-01$ & 1.34E-01 & $2.20 \mathrm{E}-01$ & $2.22 \mathrm{E}-01$ & $2.17 \mathrm{E}-01$ & $2.24 \mathrm{E}-01$ \\
\hline$\overline{\mathrm{C2O}}$ & $2.21 \mathrm{E}-02$ & $2.20 \mathrm{E}-02$ & $2.20 \mathrm{E}-02$ & $1.41 \mathrm{E}-02$ & $1.39 \mathrm{E}-02$ & $2.25 \mathrm{E}-02$ & $2.21 \mathrm{E}-02$ & $1.39 \mathrm{E}-02$ & $1.40 \mathrm{E}-02$ & $1.42 \mathrm{E}-02$ & $1.40 \mathrm{E}-02$ & $2.19 \mathrm{E}-02$ & $2.20 \mathrm{E}-02$ & $2.19 \mathrm{E}-02$ & $2.22 \mathrm{E}-02$ \\
\hline NO2 & $1.19 \mathrm{E}-01$ & $1.20 \mathrm{E}-01$ & $1.24 \mathrm{E}-01$ & $7.46 \mathrm{E}-01$ & $7.56 \mathrm{E}-01$ & $1.02 \mathrm{E}-01$ & 1.17E-01 & $7.52 \mathrm{E}-01$ & $7.48 \mathrm{E}-01$ & $7.44 \mathrm{E}-01$ & $7.40 \mathrm{E}-01$ & $1.21 \mathrm{E}-01$ & $1.24 \mathrm{E}-01$ & $1.22 \mathrm{E}-01$ & $1.09 \mathrm{E}-01$ \\
\hline NO3 & $3.76 \mathrm{E}+00$ & $3.75 \mathrm{E}+00$ & $3.76 \mathrm{E}+00$ & $2.65 \mathrm{E}+00$ & $2.63 \mathrm{E}+00$ & $3.83 \mathrm{E}+00$ & $3.78 \mathrm{E}+00$ & $2.62 \mathrm{E}+00$ & $2.63 \mathrm{E}+00$ & $2.67 \mathrm{E}+00$ & $2.66 \mathrm{E}+00$ & $3.74 \mathrm{E}+00$ & $3.74 \mathrm{E}+00$ & $3.74 \mathrm{E}+00$ & $3.80 \mathrm{E}+00$ \\
\hline $\mathrm{OH}$ & $1.02 \mathrm{E}+00$ & $1.02 \mathrm{E}+00$ & $9.85 \mathrm{E}-01$ & $1.85 \mathrm{E}+00$ & $1.87 \mathrm{E}+00$ & $9.20 \mathrm{E}-01$ & $9.75 \mathrm{E}-01$ & $1.94 \mathrm{E}+00$ & $1.89 \mathrm{E}+00$ & $1.86 \mathrm{E}+00$ & $1.86 \mathrm{E}+00$ & $1.05 \mathrm{E}+00$ & $1.07 \mathrm{E}+00$ & $9.69 \mathrm{E}-01$ & $9.45 \mathrm{E}-01$ \\
\hline $\mathrm{PO4}$ & $1.53 \mathrm{E}-03$ & $1.53 \mathrm{E}-03$ & $1.62 \mathrm{E}-03$ & $1.57 \mathrm{E}-02$ & $1.56 \mathrm{E}-02$ & $1.37 \mathrm{E}-03$ & & 1.57E-02 & $1.55 \mathrm{E}-02$ & $1.57 \mathrm{E}-02$ & $1.57 \mathrm{E}-02$ & $1.44 \mathrm{E}-03$ & $1.45 \mathrm{E}-03$ & $1.51 \mathrm{E}-03$ & $1.37 \mathrm{E}-03$ \\
\hline$\overline{\mathrm{SO4}}$ & $3.21 \mathrm{E}-01$ & $3.20 \mathrm{E}-01$ & $3.21 \mathrm{E}-01$ & $1.83 \mathrm{E}-01$ & $1.80 \mathrm{E}-01$ & $3.28 \mathrm{E}-01$ & $3.22 \mathrm{E}-01$ & $1.80 \mathrm{E}-01$ & $1.80 \mathrm{E}-01$ & $1.85 \mathrm{E}-01$ & $1.82 \mathrm{E}-01$ & $3.19 \mathrm{E}-01$ & $3.20 \mathrm{E}-01$ & $3.19 \mathrm{E}-01$ & $3.24 \mathrm{E}-01$ \\
\hline$\overline{\mathrm{Ag}}$ & $1.21 \mathrm{E}-08$ & $7.10 \mathrm{E}-08$ & $5.23 \mathrm{E}-08$ & $2.65 \mathrm{E}-08$ & $2.26 \mathrm{E}-08$ & $2.49 \mathrm{E}-08$ & $2.45 \mathrm{E}-08$ & $2.26 \mathrm{E}-08$ & $2.26 \mathrm{E}-08$ & $2.92 \mathrm{E}-08$ & $2.27 \mathrm{E}-08$ & $2.43 \mathrm{E}-08$ & $2.44 \mathrm{E}-08$ & $2.43 \mathrm{E}-08$ & $2.46 \mathrm{E}-08$ \\
\hline As & $2.13 \mathrm{E}-06$ & $1.93 \mathrm{E}-06$ & $2.51 \mathrm{E}-06$ & $3.45 \mathrm{E}-06$ & 3.55E-05 & $1.72 \mathrm{E}-05$ & 4.37E-05 & $6.17 \mathrm{E}-05$ & $4.44 \mathrm{E}-05$ & $2.38 \mathrm{E}-06$ & $2.94 \mathrm{E}-05$ & $7.38 \mathrm{E}-05$ & $8.09 \mathrm{E}-05$ & $4.45 \mathrm{E}-05$ & $3.08 \mathrm{E}-05$ \\
\hline$\overline{\mathrm{Ba}}$ & $2.11 \mathrm{E}-09$ & $2.11 \mathrm{E}-08$ & $1.58 \mathrm{E}-08$ & $1.39 \mathrm{E}-07$ & ---- & 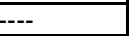 & ---- & - & --- & $4.99 \mathrm{E}-09$ & 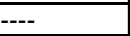 & --- & 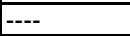 & 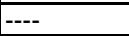 & - \\
\hline $\mathrm{Ca}$ & $4.98 \mathrm{E}-05$ & $4.97 \mathrm{E}-05$ & $4.98 \mathrm{E}-05$ & $3.17 \mathrm{E}-05$ & 3.05E-05 & $5.19 \mathrm{E}-05$ & $4.99 \mathrm{E}-05$ & $3.05 \mathrm{E}-05$ & 3.05E-05 & $\mathrm{E}-05$ & E-01 & $4.96 \mathrm{E}-05$ & $4.97 \mathrm{E}-05$ & $4.95 \mathrm{E}-05$ & $5.04 \mathrm{E}-05$ \\
\hline$\overline{C d}$ & $5.80 \mathrm{E}-08$ & $5.53 \mathrm{E}-08$ & $7.34 \mathrm{E}-08$ & $9.08 \mathrm{E}-08$ & $9.57 \mathrm{E}-07$ & $2.06 \mathrm{E}-07$ & $1.24 \mathrm{E}-06$ & $1.97 \mathrm{E}-06$ & $1.30 \mathrm{E}-06$ & $4.88 \mathrm{E}-08$ & 7.17E-07 & $2.40 \mathrm{E}-06$ & $2.68 \mathrm{E}-06$ & $1.27 \mathrm{E}-06$ & $7.37 \mathrm{E}-07$ \\
\hline$\overline{\mathrm{Cl}}$ & $4.23 \mathrm{E}-02$ & $4.22 \mathrm{E}-02$ & $4.23 \mathrm{E}-02$ & $3.84 \mathrm{E}-02$ & $3.79 \mathrm{E}-02$ & $4.29 \mathrm{E}-02$ & & $3.80 \mathrm{E}-02$ & $3.79 \mathrm{E}-02$ & 3.87E-02 & $3.81 \mathrm{E}-02$ & $4.21 \mathrm{E}-02$ & $4.23 \mathrm{E}-02$ & $4.20 \mathrm{E}-02$ & $4.24 \mathrm{E}-02$ \\
\hline Co & $3.09 \mathrm{E}-07$ & $3.15 \mathrm{E}-07$ & $2.60 \mathrm{E}-07$ & $1.37 \mathrm{E}-07$ & $2.02 \mathrm{E}-07$ & $1.32 \mathrm{E}-07$ & & $3.11 \mathrm{E}-07$ & $\mathrm{E}-07$ & 3E-07 & E-07 & $5 \mathrm{E}-07$ & $3.95 \mathrm{E}-07$ & $4 \mathrm{E}-07$ & $1.88 \mathrm{E}-07$ \\
\hline$\overline{\mathrm{Cr}}$ & $1.28 \mathrm{E}-05$ & $7.79 \mathrm{E}-06$ & $2.59 \mathrm{E}-05$ & $1.06 \mathrm{E}-04$ & $1.42 \mathrm{E}-04$ & $1.75 \mathrm{E}-04$ & & $1.21 \mathrm{E}-04$ & $1.35 \mathrm{E}-04$ & $1.34 \mathrm{E}-04$ & $1.48 \mathrm{E}-04$ & $1.24 \mathrm{E}-04$ & $1.19 \mathrm{E}-04$ & $1.48 \mathrm{E}-04$ & $1.61 \mathrm{E}-04$ \\
\hline $\mathrm{Cu}$ & $1.11 \mathrm{E}-04$ & $1.15 \mathrm{E}-04$ & $8.39 \mathrm{E}-05$ & $2.00 \mathrm{E}-05$ & $5.68 \mathrm{E}-05$ & $1.23 \mathrm{E}-05$ & -05 & $1.17 \mathrm{E}-04$ & $7.71 \mathrm{E}-05$ & $1.71 \mathrm{E}-05$ & $25 \mathrm{E}-05$ & $1.42 \mathrm{E}-04$ & $1.59 \mathrm{E}-04$ & $7.54 \mathrm{E}-05$ & $4.38 \mathrm{E}-05$ \\
\hline $\mathrm{Fe}$ & $9.34 \mathrm{E}-07$ & $9.32 \mathrm{E}-07$ & $7.26 \mathrm{E}-07$ & $5.60 \mathrm{E}-07$ & $2.58 \mathrm{E}-06$ & $6.30 \mathrm{E}-07$ & $3.32 \mathrm{E}-06$ & $5.23 \mathrm{E}-06$ & $3.48 \mathrm{E}-06$ & $1.30 \mathrm{E}-06$ & $1.96 \mathrm{E}-06$ & $6.37 \mathrm{E}-06$ & $7.08 \mathrm{E}-06$ & $3.41 \mathrm{E}-06$ & $2.02 \mathrm{E}-06$ \\
\hline $\mathbf{F}$ & $7.04 \mathrm{E}-02$ & $7.02 \mathrm{E}-02$ & $7.03 \mathrm{E}-02$ & $3.61 \mathrm{E}-02$ & $3.56 \mathrm{E}-02$ & $7.17 \mathrm{E}-02$ & & $3.56 \mathrm{E}-02$ & $E-02$ & $\mathrm{E}-02$ & E-02 & $7.00 \mathrm{E}-02$ & $7.02 \mathrm{E}-02$ & $3 \mathrm{E}-02$ & $7.09 \mathrm{E}-02$ \\
\hline $\mathrm{Hg}$ & $7.23 \mathrm{E}-07$ & $7.21 \mathrm{E}-07$ & $7.22 \mathrm{E}-07$ & $1.36 \mathrm{E}-06$ & $8.20 \mathrm{E}-06$ & $8.61 \mathrm{E}-06$ & & $8.74 \mathrm{E}-06$ & E-06 & $3 \mathrm{E}-07$ & $2 \mathrm{E}-06$ & $7 \mathrm{E}-06$ & $9.73 \mathrm{E}-06$ & $8.95 \mathrm{E}-06$ & $8.79 \mathrm{E}-06$ \\
\hline $\mathbf{K}$ & $6.61 \mathrm{E}-03$ & $6.74 \mathrm{E}-03$ & $6.60 \mathrm{E}-03$ & $4.99 \mathrm{E}-03$ & $5.38 \mathrm{E}-03$ & $5.01 \mathrm{E}-03$ & $E-03$ & $6.64 \mathrm{E}-03$ & $5.53 \mathrm{E}-03$ & $4.65 \mathrm{E}-03$ & $4.88 \mathrm{E}-03$ & $7.01 \mathrm{E}-03$ & $7.31 \mathrm{E}-03$ & $5.93 \mathrm{E}-03$ & $5.55 \mathrm{E}-03$ \\
\hline$\overline{\mathrm{Mg}}$ & 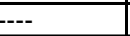 & --- & $6.80 \mathrm{E}-09$ & $8.76 \mathrm{E}-08$ & $3.49 \mathrm{E}-08$ & $1.08 \mathrm{E}-07$ & $2.19 \mathrm{E}-08$ & 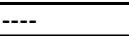 & $6.92 \mathrm{E}-09$ & $9.24 \mathrm{E}-08$ & $5.53 \mathrm{E}-08$ & $-{ }_{---}$ & ---- & $1.78 \mathrm{E}-08$ & $6.32 \mathrm{E}-08$ \\
\hline$M n$ & $3.96 \mathrm{E}-08$ & $4.74 \mathrm{E}-08$ & $6.32 \mathrm{E}-08$ & $7.43 \mathrm{E}-08$ & 1.34E-07 & $6.99 \mathrm{E}-08$ & $1.63 \mathrm{E}-07$ & $2.26 \mathrm{E}-07$ & $1.65 \mathrm{E}-07$ & $3.99 \mathrm{E}-08$ & $1.12 \mathrm{E}-07$ & \begin{tabular}{|c|}
$2.69 \mathrm{E}-07$ \\
\end{tabular} & $2.94 \mathrm{E}-07$ & $1.66 \mathrm{E}-07$ & $1.18 \mathrm{E}-07$ \\
\hline Nd & --- & $-{ }_{-1}$ & ---- & $2.91 \mathrm{E}-06$ & ---- & $3.91 \mathrm{E}-06$ & --- & --- & ---- & E-06 & E-06 & --- & ---- & ---- & $1.35 \mathrm{E}-06$ \\
\hline$\overline{\mathrm{Ni}}$ & $7.40 \mathrm{E}-07$ & $7.57 \mathrm{E}-07$ & $6.09 \mathrm{E}-07$ & $2.88 \mathrm{E}-07$ & $4.63 \mathrm{E}-07$ & $2.68 \mathrm{E}-07$ & $5.59 \mathrm{E}-07$ & $7.52 \mathrm{E}-07$ & $5.61 \mathrm{E}-07$ & $2.76 \mathrm{E}-07$ & $3.95 \mathrm{E}-07$ & $8.91 \mathrm{E}-07$ & $9.69 \mathrm{E}-07$ & $5.67 \mathrm{E}-07$ & $4.17 \mathrm{E}-07$ \\
\hline$\overline{\mathrm{Pb}}$ & $2.03 \mathrm{E}-06$ & $1.67 \mathrm{E}-06$ & $2.31 \mathrm{E}-06$ & $3.35 \mathrm{E}-06$ & ---- & -- & ---- & --- & --- & $1.98 \mathrm{E}-06$ & ---- & ---- & $\begin{array}{ll}--- \\
\end{array}$ & ---- & --- \\
\hline $\mathbf{R u}$ & $1.17 \mathrm{E}-05$ & $1.21 \mathrm{E}-05$ & $9.07 \mathrm{E}-06$ & $2.78 \mathrm{E}-06$ & $6.36 \mathrm{E}-06$ & $2.10 \mathrm{E}-06$ & $8.04 \mathrm{E}-06$ & $1.22 \mathrm{E}-05$ & $8.34 \mathrm{E}-06$ & $2.51 \mathrm{E}-06$ & $4.97 \mathrm{E}-06$ & $1.48 \mathrm{E}-05$ & 1.64E-05 & $8.24 \mathrm{E}-06$ & $5.16 \mathrm{E}-06$ \\
\hline$\overline{\mathrm{Se}}$ & $1.51 \mathrm{E}-05$ & $1.15 \mathrm{E}-05$ & $1.94 \mathrm{E}-05$ & $4.05 \mathrm{E}-05$ & $1.09 \mathrm{E}-04$ & $1.46 \mathrm{E}-04$ & $1.13 \mathrm{E}-04$ & $7.90 \mathrm{E}-05$ & $9.89 \mathrm{E}-05$ & $3.90 \mathrm{E}-05$ & $1.17 \mathrm{E}-04$ & $7.68 \mathrm{E}-05$ & $6.90 \mathrm{E}-05$ & $1.10 \mathrm{E}-04$ & $1.28 \mathrm{E}-04$ \\
\hline$\overline{\mathrm{Si}}$ & $7.17 \mathrm{E}-05$ & $6.28 \mathrm{E}-05$ & $3.68 \mathrm{E}-04$ & $1.21 \mathrm{E}-05$ & ---- & --- &.--- & 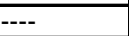 & ---- & $9.51 \mathrm{E}-05$ & -.- & --- & --- & --- & --- \\
\hline $\mathrm{Sr}$ & --- & --- & ---- & $1.96 \mathrm{E}-06$ & --- & $2.71 \mathrm{E}-06$ & --- & - & --- & $2.16 \mathrm{E}-06$ & $5.35 \mathrm{E}-07$ & - & --- & --- & $7.27 \mathrm{E}-07$ \\
\hline$Z n$ & $1.35 \mathrm{E}-04$ & $1.39 \mathrm{E}-04$ & \begin{tabular}{|r|}
$1.05 \mathrm{E}-04$ \\
\end{tabular} & $3.51 \mathrm{E}-05$ & $7.48 \mathrm{E}-05$ & $2.79 \mathrm{E}-05$ & $9.39 \mathrm{E}-05$ & $1.40 \mathrm{E}-04$ & $9.69 \mathrm{E}-05$ & $3.20 \mathrm{E}-05$ & 5.94E-05 & \begin{tabular}{|c|}
$1.69 \mathrm{E}-04$ \\
\end{tabular} & $1.86 \mathrm{E}-04$ & $9.61 \mathrm{E}-05$ & $6.19 \mathrm{E}-05$ \\
\hline$\overline{Z r}$ & $3.55 \mathrm{E}-06$ & $3.66 \mathrm{E}-06$ & $2.70 \mathrm{E}-06$ & $7.09 \mathrm{E}-07$ & $1.85 \mathrm{E}-06$ & $4.76 \mathrm{E}-07$ & 2.37E-06 & $3.72 \mathrm{E}-06$ & $2.48 \mathrm{E}-06$ & $6.19 \mathrm{E}-07$ & $1.41 \mathrm{E}-06$ & $4.52 \mathrm{E}-06$ & $5.03 \mathrm{E}-06$ & $2.44 \mathrm{E}-06$ & $1.46 \mathrm{E}-06$ \\
\hline Insol.Sol.mg/L & 0 & 0 & 0 & 0 & 0 & 0 & 0 & 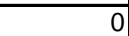 & 0 & 0 & 0 & & 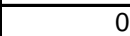 & & \\
\hline
\end{tabular}


Table 11: Projected Feed Composition After Dissolution and Dilution to 6.44 M Total Sodium Concentration no sludge; $20 \%$ of interstitial supernate remaining (continued)

\begin{tabular}{|c|c|c|c|c|c|c|c|c|c|c|c|c|c|c|c|}
\hline \multicolumn{16}{|c|}{ Radionuclide Composition (Ci/gal) } \\
\hline & Tank 25 & \begin{tabular}{|l|} 
Tank 27 \\
\end{tabular} & \begin{tabular}{|l} 
Tank 28 \\
\end{tabular} & Tank 29 & Tank 31 & \begin{tabular}{|c|} 
Tank 33 \\
\end{tabular} & Tank 34 & Tank 36 & \begin{tabular}{|l|} 
Tank 37 \\
\end{tabular} & Tank 38 & Tank 41 & Tank 44 & Tank 45 & Tank 46 & Tank 47 \\
\hline C-14 & 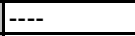 & --- & ---- & \begin{tabular}{|l|}
--- \\
\end{tabular} & \begin{tabular}{|l|}
--- \\
\end{tabular} & ---- & --- & ---- & $---\cdot$ & --- & ---- & --- & 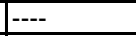 & \begin{tabular}{|l|}
--- \\
\end{tabular} & --- \\
\hline $\mathrm{Ni}-59$ & --- & --- & --- & --- & --- & ---- & --- & --- & ---- & ---- & --- & --- & --- & --- & ---- \\
\hline $\mathrm{Ni}-63$ & --- & --- & --- & --- & --- & ---- & --- & --- & ---- & --- & --- & --- & --- & --- & --- \\
\hline Co-60 & ---- & ---- & ---- & $-\cdots-$ & $-\cdots-$ & $-\cdots-$ & $-\cdots$ & $-\cdots$ & ---- & $-\cdots-$ & \begin{tabular}{|c|}
--- \\
\end{tabular} & $-\cdots-$ & $-\cdots-$ & ---- & --- \\
\hline Se-79 & $-\cdots$ & -- & $-{ }_{-}$ & 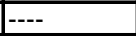 & --- & --- & --- & --- & --- & $-{ }_{-1}$ & $-{ }_{---}$ & $-{ }_{---}$ & - & -- & 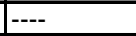 \\
\hline TC-99 & $-\cdots$ & --- & --- & --- & --- & --- & --- & --- & --- & --- & $-{ }_{---}$ & --- & - & --- & --- \\
\hline Ru-106 & --- & --- & $-\cdots$ & $-\cdots$ & --- & ---- & \begin{tabular}{|c|}
--- \\
\end{tabular} & --- & \begin{tabular}{|c|}
--- \\
\end{tabular} & 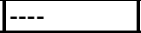 & --- & --- & --- & --- & --- \\
\hline Rh-106 & $-\cdots$ & ---- & ---- & $-\cdots$ & $-\cdots$ & $-\cdots$ & --- & $-\cdots$ & ---- & ---- & --- & $-\cdots$ & $-\cdots$ & --- & ---- \\
\hline Sb-125 & - & --- & --- & --- & - & --- & --- & --- & --- & --- & --- & - & - & --- & --- \\
\hline Sn-126 & --- & --- & --- & --- & --- & --- & --- & --- & --- & --- & --- & 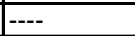 & - & --- & ---- \\
\hline $1-129$ & $-{ }_{-1}$ & --- & ---- & $-\cdots$ & --- & --- & --- & $-\cdots$ & --- & --- & $-\cdots$ & --- & --- & --- & --- \\
\hline Cs-134 & ---- & ---- & ---- & $-\cdots$ & $-\cdots-$ & ---- & ---- & $-\cdots$ & ---- & ---- & --- & $-\cdots$ & $-\cdots$ & ---- & \begin{tabular}{|c|}
--- \\
\end{tabular} \\
\hline Cs-135 & \begin{tabular}{|l|}
--- \\
\end{tabular} & $\mid---$ & $\mid---$ & ---- & ---- & \begin{tabular}{|l|}
---- \\
\end{tabular} & \begin{tabular}{|l|}
--- \\
\end{tabular} & \begin{tabular}{|l|}
--- \\
\end{tabular} & \begin{tabular}{|l|}
--- \\
\end{tabular} & \begin{tabular}{|l|}
--- \\
\end{tabular} & \begin{tabular}{|l|}
---- \\
\end{tabular} & \begin{tabular}{|l|}
---- \\
\end{tabular} & $\mid---$ & ---- & \begin{tabular}{|l|}
---- \\
\end{tabular} \\
\hline Cs-137 & \begin{tabular}{|c|}
$6.22 \mathrm{E}-02$ \\
\end{tabular} & \begin{tabular}{|r}
$5.89 \mathrm{E}-02$ \\
\end{tabular} & \begin{tabular}{|r|}
$6.47 \mathrm{E}-02$ \\
\end{tabular} & \begin{tabular}{|r}
$5.57 \mathrm{E}-02$ \\
\end{tabular} & $1.52 \mathrm{E}-01$ & 1.47E-03 & 5.06E-02 & $2.80 \mathrm{E}-01$ & $2.03 \mathrm{E}-01$ & 3.71E-03 & 3.40E-02 & 7.64E-02 & $7.24 \mathrm{E}-02$ & $9.31 \mathrm{E}-02$ & $3.90 \mathrm{E}-02$ \\
\hline$B a-137 m$ & $5.88 \mathrm{E}-02$ & $5.57 \mathrm{E}-02$ & $6.12 \mathrm{E}-02$ & 5.27E-02 & $1.44 \mathrm{E}-01$ & $1.39 \mathrm{E}-03$ & $4.78 \mathrm{E}-02$ & $2.65 \mathrm{E}-01$ & $1.92 \mathrm{E}-01$ & $3.51 \mathrm{E}-03$ & $3.21 \mathrm{E}-02$ & $7.23 \mathrm{E}-02$ & $6.85 \mathrm{E}-02$ & $8.81 \mathrm{E}-02$ & $3.69 \mathrm{E}-02$ \\
\hline Sr-90 & $4.20 \mathrm{E}-05$ & $4.19 \mathrm{E}-05$ & $4.20 \mathrm{E}-05$ & $1.50 \mathrm{E}-04$ & 1.47E-04 & $1.63 \mathrm{E}-04$ & $1.60 \mathrm{E}-04$ & $1.47 \mathrm{E}-04$ & $1.47 \mathrm{E}-04$ & $3.97 \mathrm{E}-05$ & $3.90 \mathrm{E}-05$ & $4.18 \mathrm{E}-05$ & $4.19 \mathrm{E}-05$ & $4.17 \mathrm{E}-05$ & $4.23 \mathrm{E}-05$ \\
\hline$Y-90$ & 4.20E-05 & 4.19E-05 & \begin{tabular}{|c|}
$4.20 \mathrm{E}-05$ \\
\end{tabular} & 1.50E-04 & 1.47E-04 & 1.63E-04 & $1.60 \mathrm{E}-04$ & 1.47E-04 & 1.47E-04 & 3.97E-05 & 3.90E-05 & 4.18E-05 & 4.19E-05 & 4.17E-05 & $4.23 \mathrm{E}-05$ \\
\hline $\mathrm{H}-3$ & \begin{tabular}{|l|}
--- \\
\end{tabular} & \begin{tabular}{|l|}
--- \\
\end{tabular} & \begin{tabular}{|l|}
--- \\
\end{tabular} & $\mid----$ & ---- & \begin{tabular}{|l|}
--- \\
\end{tabular} & \begin{tabular}{|l|}
--- \\
\end{tabular} & \begin{tabular}{|l|}
--- \\
\end{tabular} & \begin{tabular}{|l|}
--- \\
\end{tabular} & \begin{tabular}{|l|}
--- \\
\end{tabular} & \begin{tabular}{|l|}
---- \\
\end{tabular} & \begin{tabular}{|l|}
--- \\
\end{tabular} & ---- & $\mid----$ & ---- \\
\hline Ce-144 & --- & --- & $-\cdots$ & --- & --- & $-{ }_{---}$ & --- & --- & ---- & --- & --- & --- & - & --- & --- \\
\hline Pr-144 & --- & --- & --- & $-{ }_{-1}$ & --- & --- & $-{ }_{---}$ & $-\cdots$ & ---- & ---- & --- & $-{ }_{---}$ & --- & --- & $-{ }_{---}$ \\
\hline Pm-147 & --- & $-{ }_{---}$ & ---- & \begin{tabular}{|c|}
--- \\
\end{tabular} & $-\cdots-$ & $-\cdots-$ & $-\cdots-$ & $-\cdots$ & --- & $-\cdots$ & \begin{tabular}{|c|}
--- \\
\end{tabular} & $-\cdots-$ & --- & ---- & \begin{tabular}{|l|}
--- \\
\end{tabular} \\
\hline Eu-154 & - & --- & --- & ---- & \begin{tabular}{|l|}
--- \\
\end{tabular} & --- & --- & \begin{tabular}{|l|}
--- \\
\end{tabular} & \begin{tabular}{|l|}
--- \\
\end{tabular} & --- & - & - & - & --- & --- \\
\hline Th-232 & --- & --- & $-{ }_{---}$ & $7.33 \mathrm{E}-12$ & $7.21 \mathrm{E}-12$ & --- & --- & \begin{tabular}{|c|}
$7.21 \mathrm{E}-12$ \\
\end{tabular} & $7.21 \mathrm{E}-12$ & --- & --- & --- & --- & ---- & --- \\
\hline $\mathrm{U}-232$ & $5.05 \mathrm{E}-11$ & $5.04 \mathrm{E}-11$ & 5.04E-11 & $2.08 \mathrm{E}-12$ & $2.05 \mathrm{E}-12$ & 6.79E-10 & 6.77E-10 & $2.05 \mathrm{E}-12$ & $2.05 \mathrm{E}-12$ & $-{ }^{---}$ & --- & \begin{tabular}{|l|}
$5.02 \mathrm{E}-11$ \\
\end{tabular} & $5.03 \mathrm{E}-11$ & $5.01 \mathrm{E}-11$ & $5.09 \mathrm{E}-11$ \\
\hline U-233 & \begin{tabular}{|l|}
--- \\
\end{tabular} & $\mid---$ & $\mid---$ & $1.50 \mathrm{E}-09$ & $1.48 \mathrm{E}-09$ & \begin{tabular}{|l|}
--- \\
\end{tabular} & $\mid---$ & $1.48 \mathrm{E}-09$ & $1.48 \mathrm{E}-09$ & 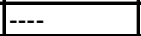 & \begin{tabular}{|l|}
--- \\
\end{tabular} & $\mid---$ & $\mid---$ & $\mid---$ & ---- \\
\hline U-234 & --- & --- & $-\cdots$ & $2.12 \mathrm{E}-10$ & $2.09 \mathrm{E}-10$ & --- & --- & $2.09 \mathrm{E}-10$ & $2.09 \mathrm{E}-10$ & $3.49 \mathrm{E}-09$ & \begin{tabular}{|l}
$3.43 \mathrm{E}-09$ \\
\end{tabular} & --- & - & --- & --- \\
\hline U-235 & $1.13 \mathrm{E}-10$ & $1.13 \mathrm{E}-10$ & $1.13 \mathrm{E}-10$ & $5.80 \mathrm{E}-12$ & $5.70 \mathrm{E}-12$ & $8.04 \mathrm{E}-10$ & $6.34 \mathrm{E}-10$ & $5.70 \mathrm{E}-12$ & $5.70 \mathrm{E}-12$ & $3.52 \mathrm{E}-11$ & $3.45 \mathrm{E}-11$ & $1.12 \mathrm{E}-10$ & $1.13 \mathrm{E}-10$ & $1.12 \mathrm{E}-10$ & $1.14 \mathrm{E}-10$ \\
\hline U-236 & $\begin{array}{ll}--- \\
\end{array}$ & $\mid---$ & $\mid---$ & $2.19 \mathrm{E}-11$ & $2.15 \mathrm{E}-11$ & \begin{tabular}{|l|}
--- \\
\end{tabular} & \begin{tabular}{|l|}
--- \\
\end{tabular} & $2.15 \mathrm{E}-11$ & $2.15 \mathrm{E}-11$ & $5.32 \mathrm{E}-10$ & $5.22 \mathrm{E}-10$ & $\mid----$ & ---- & $\mid---$ & $\mid----$ \\
\hline $\mathrm{U}-238$ & \begin{tabular}{|c|}
$1.03 \mathrm{E}-08$ \\
\end{tabular} & $1.03 \mathrm{E}-08$ & 1.03E-08 & $6.78 \mathrm{E}-11$ & $6.66 \mathrm{E}-11$ & \begin{tabular}{|c|}
$7.35 \mathrm{E}-08$ \\
\end{tabular} & $4.48 \mathrm{E}-08$ & $6.66 \mathrm{E}-11$ & $6.66 \mathrm{E}-11$ & $4.94 \mathrm{E}-12$ & $4.85 \mathrm{E}-12$ & 1.03E-08 & $1.03 \mathrm{E}-08$ & $1.02 \mathrm{E}-08$ & $1.04 \mathrm{E}-08$ \\
\hline Np-237 & $\begin{array}{ll}--- \\
\end{array}$ & $\mid---$ & \begin{tabular}{|l|}
--- \\
\end{tabular} & $5.45 \mathrm{E}-10$ & $5.36 \mathrm{E}-10$ & 6.06E-08 & 3.46E-08 & $5.36 \mathrm{E}-10$ & $5.36 \mathrm{E}-10$ & $3.04 \mathrm{E}-09$ & $2.99 \mathrm{E}-09$ & \begin{tabular}{|l|}
--- \\
\end{tabular} & ---- & $\mid---$ & ---- \\
\hline Pu-238 & $8.55 \mathrm{E}-05$ & $8.53 \mathrm{E}-05$ & \begin{tabular}{|c|}
$8.54 \mathrm{E}-05$ \\
\end{tabular} & $2.08 \mathrm{E}-06$ & $2.05 \mathrm{E}-06$ & \begin{tabular}{|l|}
---- \\
\end{tabular} & $\mid---$ & $2.05 \mathrm{E}-06$ & $2.05 \mathrm{E}-06$ & $9.60 \mathrm{E}-05$ & $9.43 \mathrm{E}-05$ & $8.51 \mathrm{E}-05$ & $8.52 \mathrm{E}-05$ & $8.49 \mathrm{E}-05$ & $8.61 \mathrm{E}-05$ \\
\hline Pu-239 & $1.22 \mathrm{E}-05$ & $1.22 \mathrm{E}-05$ & $1.22 \mathrm{E}-05$ & $5.39 \mathrm{E}-08$ & $5.31 \mathrm{E}-08$ & $8.80 \mathrm{E}-06$ & $7.23 \mathrm{E}-06$ & $5.31 \mathrm{E}-08$ & $5.31 \mathrm{E}-08$ & $2.96 \mathrm{E}-07$ & $2.90 \mathrm{E}-07$ & $1.22 \mathrm{E}-05$ & $1.22 \mathrm{E}-05$ & $1.21 \mathrm{E}-05$ & $1.23 \mathrm{E}-05$ \\
\hline Pu-240 & $2.73 \mathrm{E}-06$ & $2.72 \mathrm{E}-06$ & $2.73 \mathrm{E}-06$ & $2.23 \mathrm{E}-08$ & $2.19 \mathrm{E}-08$ & 1.97E-06 & $1.62 \mathrm{E}-06$ & $2.19 \mathrm{E}-08$ & $2.19 \mathrm{E}-08$ & $1.95 \mathrm{E}-07$ & 1.92E-07 & $2.72 \mathrm{E}-06$ & $2.72 \mathrm{E}-06$ & $2.71 \mathrm{E}-06$ & $2.75 \mathrm{E}-06$ \\
\hline Pu-241 & $\begin{array}{l}7.33 \mathrm{E}-05 \\
\end{array}$ & $7.31 \mathrm{E}-05$ & $7.32 \mathrm{E}-05$ & 4.84E-07 & $4.76 \mathrm{E}-07$ & $5.12 \mathrm{E}-05$ & $4.12 \mathrm{E}-05$ & $4.76 \mathrm{E}-07$ & $4.76 \mathrm{E}-07$ & $1.63 \mathrm{E}-05$ & $1.61 \mathrm{E}-05$ & $7.29 \mathrm{E}-05$ & $\begin{array}{l}7.30 \mathrm{E}-05 \\
\end{array}$ & $7.27 \mathrm{E}-05$ & $7.38 \mathrm{E}-05$ \\
\hline Pu-242 & $5.62 \mathrm{E}-10$ & \begin{tabular}{|r|}
$5.60 \mathrm{E}-10$ \\
\end{tabular} & \begin{tabular}{|l|}
$5.61 \mathrm{E}-10$ \\
\end{tabular} & $1.48 \mathrm{E}-11$ & $1.46 \mathrm{E}-11$ & $4.05 \mathrm{E}-10$ & $3.35 \mathrm{E}-10$ & $1.46 \mathrm{E}-11$ & $1.46 \mathrm{E}-11$ & 3.67E-09 & $3.60 \mathrm{E}-09$ & $5.59 \mathrm{E}-10$ & $5.60 \mathrm{E}-10$ & $5.58 \mathrm{E}-10$ & $5.66 \mathrm{E}-10$ \\
\hline Am-241 & \begin{tabular}{|l|}
--- \\
\end{tabular} & $\mid---$ & \begin{tabular}{|l|}
--- \\
\end{tabular} & $6.13 \mathrm{E}-07$ & $6.03 \mathrm{E}-07$ & $9.22 \mathrm{E}-05$ & $9.27 \mathrm{E}-05$ & $6.03 \mathrm{E}-07$ & $6.03 \mathrm{E}-07$ & $5.12 \mathrm{E}-07$ & $5.03 \mathrm{E}-07$ & $\mid---$ & ---- & $\mid---$ & ---- \\
\hline$A m-242 m$ & $-{ }_{-1}$ & --- & $-{ }_{---}$ & $7.48 \mathrm{E}-10$ & $7.36 \mathrm{E}-10$ & $1.26 \mathrm{E}-07$ & $1.26 \mathrm{E}-07$ & $7.36 \mathrm{E}-10$ & $7.36 \mathrm{E}-10$ & $6.38 \mathrm{E}-10$ & $6.26 \mathrm{E}-10$ & --- & --- & $-{ }_{-1}$ & - \\
\hline$\overline{C m-242}$ & $-\cdots$ & $-\cdots$ & $-\cdots$ & --- & \begin{tabular}{|l|}
--- \\
\end{tabular} & \begin{tabular}{|l|}
--- \\
\end{tabular} & \begin{tabular}{|l|}
--- \\
\end{tabular} & $-\cdots$ & \begin{tabular}{|l|}
--- \\
\end{tabular} & \begin{tabular}{|l|}
--- \\
\end{tabular} & \begin{tabular}{|l}
--- \\
\end{tabular} & --- & --- & - & --- \\
\hline Cm-244 & $1.06 \mathrm{E}-09$ & $1.06 \mathrm{E}-09$ & 1.06E-09 & $1.79 \mathrm{E}-09$ & 1.77E-09 & $4.89 \mathrm{E}-08$ & $4.73 \mathrm{E}-08$ & 1.77E-09 & 1.77E-09 & 8.65E-09 & 8.50E-09 & 1.06E-09 & $1.06 \mathrm{E}-09$ & $1.05 \mathrm{E}-09$ & 1.07E-09 \\
\hline$\overline{C m-245}$ & $4.18 \mathrm{E}-16$ & $4.17 \mathrm{E}-16$ & $4.18 \mathrm{E}-16$ & $2.21 \mathrm{E}-13$ & $2.18 \mathrm{E}-13$ & $1.93 \mathrm{E}-14$ & $1.94 \mathrm{E}-14$ & $2.18 \mathrm{E}-13$ & $2.18 \mathrm{E}-13$ & $5.32 \mathrm{E}-13$ & $5.23 \mathrm{E}-13$ & $4.16 \mathrm{E}-16$ & $4.17 \mathrm{E}-16$ & $4.15 \mathrm{E}-16$ & $4.21 \mathrm{E}-16$ \\
\hline Total Alpha & $1.00 \mathrm{E}-04$ & $1.00 \mathrm{E}-04$ & $1.00 \mathrm{E}-04$ & $2.78 \mathrm{E}-06$ & $2.73 \mathrm{E}-06$ & $1.03 \mathrm{E}-04$ & $1.02 \mathrm{E}-04$ & $2.73 \mathrm{E}-06$ & $2.73 \mathrm{E}-06$ & $9.70 \mathrm{E}-05$ & $9.53 \mathrm{E}-05$ & $1.00 \mathrm{E}-04$ & $1.00 \mathrm{E}-04$ & $9.97 \mathrm{E}-05$ & $1.01 \mathrm{E}-04$ \\
\hline
\end{tabular}


Table 12: Projected Feed Composition After Dissolution and Dilution to 6.44 M Total Sodium Concentration $600 \mathrm{mg} / \mathrm{L}$ sludge; $10 \%$ of interstitial supernate remaining

\begin{tabular}{|c|c|c|c|c|c|c|c|c|c|c|c|c|c|c|c|}
\hline \multicolumn{16}{|c|}{ Concentration (M) } \\
\hline & Tank 25 & Tank 27 & Tank 28 & Tank 29 & Tank 31 & Tank 33 & Tank 34 & Tank 36 & Tank 37 & Tank 38 & Tank 41 & Tank 44 & Tank 45 & Tank 46 & Tank 47 \\
\hline $\mathrm{Na}$ & $6.44 \mathrm{E}+00$ & $6.44 \mathrm{E}+00$ & $6.44 \mathrm{E}+00$ & $6.44 \mathrm{E}+00$ & $6.44 \mathrm{E}+00$ & $6.44 \mathrm{E}+00$ & $6.44 \mathrm{E}+00$ & $6.44 \mathrm{E}+00$ & $6.44 \mathrm{E}+00$ & $6.44 \mathrm{E}+00$ & $6.44 \mathrm{E}+00$ & $6.44 \mathrm{E}+00$ & $6.44 \mathrm{E}+00$ & $6.44 \mathrm{E}+00$ & $6.44 \mathrm{E}+00$ \\
\hline $\mathrm{Al}(\mathrm{OH}) 4$ & $3.22 \mathrm{E}-01$ & $3.25 \mathrm{E}-01$ & $3.22 \mathrm{E}-01$ & $3.99 \mathrm{E}-01$ & $4.00 \mathrm{E}-01$ & $3.21 \mathrm{E}-01$ & $3.20 \mathrm{E}-01$ & $3.94 \mathrm{E}-01$ & 3.97E-01 & $4.00 \mathrm{E}-01$ & 3.96E-01 & $3.20 \mathrm{E}-01$ & $3.19 \mathrm{E}-01$ & $3.17 \mathrm{E}-01$ & $3.22 \mathrm{E}-01$ \\
\hline $\mathrm{CO} 3$ & $2.22 \mathrm{E}-01$ & $2.22 \mathrm{E}-01$ & $2.21 \mathrm{E}-01$ & $1.33 \mathrm{E}-01$ & $1.32 \mathrm{E}-01$ & $2.23 \mathrm{E}-01$ & $2.25 \mathrm{E}-01$ & $1.33 \mathrm{E}-01$ & $1.32 \mathrm{E}-01$ & $1.34 \mathrm{E}-01$ & 1.34E-01 & $2.22 \mathrm{E}-01$ & $2.23 \mathrm{E}-01$ & $2.20 \mathrm{E}-01$ & $2.24 \mathrm{E}-01$ \\
\hline$\overline{\mathrm{C2O}}$ & $2.23 \mathrm{E}-02$ & $2.23 \mathrm{E}-02$ & $2.23 \mathrm{E}-02$ & $1.42 \mathrm{E}-02$ & $1.41 \mathrm{E}-02$ & $2.25 \mathrm{E}-02$ & $2.23 \mathrm{E}-02$ & $1.41 \mathrm{E}-02$ & $1.41 \mathrm{E}-02$ & $1.42 \mathrm{E}-02$ & $1.41 \mathrm{E}-02$ & $2.22 \mathrm{E}-02$ & $2.23 \mathrm{E}-02$ & $2.22 \mathrm{E}-02$ & $2.24 \mathrm{E}-02$ \\
\hline NO2 & $1.11 \mathrm{E}-01$ & $1.11 \mathrm{E}-01$ & $1.14 \mathrm{E}-01$ & $7.44 \mathrm{E}-01$ & $7.49 \mathrm{E}-01$ & $1.02 \mathrm{E}-01$ & $1.10 \mathrm{E}-01$ & 7.47E-01 & $7.45 \mathrm{E}-01$ & $7.43 \mathrm{E}-01$ & $7.41 \mathrm{E}-01$ & $1.12 \mathrm{E}-01$ & $1.13 \mathrm{E}-01$ & $1.12 \mathrm{E}-01$ & $1.06 \mathrm{E}-01$ \\
\hline$\overline{\mathrm{NO} 3}$ & $3.80 \mathrm{E}+00$ & $3.80 \mathrm{E}+00$ & $3.80 \mathrm{E}+00$ & $2.66 \mathrm{E}+00$ & $2.65 \mathrm{E}+00$ & $3.84 \mathrm{E}+00$ & $3.81 \mathrm{E}+00$ & $2.65 \mathrm{E}+00$ & $2.65 \mathrm{E}+00$ & $2.67 \mathrm{E}+00$ & $2.67 \mathrm{E}+00$ & $3.79 \mathrm{E}+00$ & $3.79 \mathrm{E}+00$ & $3.79 \mathrm{E}+00$ & $3.82 \mathrm{E}+00$ \\
\hline$\overline{\mathrm{OH}}$ & $9.63 \mathrm{E}-01$ & $9.65 \mathrm{E}-01$ & $9.47 \mathrm{E}-01$ & $1.85 \mathrm{E}+00$ & $1.86 \mathrm{E}+00$ & $9.14 \mathrm{E}-01$ & & $1.89 \mathrm{E}+00$ & $1.87 \mathrm{E}+00$ & $1.85 \mathrm{E}+00$ & $1.85 \mathrm{E}+00$ & $9.80 \mathrm{E}-01$ & $9.90 \mathrm{E}-01$ & $9.39 \mathrm{E}-01$ & $9.27 \mathrm{E}-01$ \\
\hline PO4 & $1.42 \mathrm{E}-03$ & $1.42 \mathrm{E}-03$ & $1.46 \mathrm{E}-03$ & $1.57 \mathrm{E}-02$ & 1.57E-02 & $1.34 \mathrm{E}-03$ & $1.34 \mathrm{E}-03$ & $1.57 \mathrm{E}-02$ & $1.56 \mathrm{E}-02$ & $1.57 \mathrm{E}-02$ & $1.57 \mathrm{E}-02$ & $1.37 \mathrm{E}-03$ & $1.38 \mathrm{E}-03$ & $1.41 \mathrm{E}-03$ & $1.34 \mathrm{E}-03$ \\
\hline SO4 & $3.25 \mathrm{E}-01$ & $3.25 \mathrm{E}-01$ & $3.25 \mathrm{E}-01$ & $1.84 \mathrm{E}-01$ & $1.83 \mathrm{E}-01$ & $3.29 \mathrm{E}-01$ & $3.26 \mathrm{E}-01$ & $1.83 \mathrm{E}-01$ & $1.83 \mathrm{E}-01$ & $1.85 \mathrm{E}-01$ & $1.83 \mathrm{E}-01$ & $3.24 \mathrm{E}-01$ & $3.25 \mathrm{E}-01$ & $3.24 \mathrm{E}-01$ & $3.27 \mathrm{E}-01$ \\
\hline$\overline{\mathrm{Ag}}$ & $6.13 \mathrm{E}-09$ & $3.60 \mathrm{E}-08$ & $2.65 \mathrm{E}-08$ & $1.33 \mathrm{E}-08$ & $1.14 \mathrm{E}-08$ & $1.25 \mathrm{E}-08$ & $1.24 \mathrm{E}-08$ & $1.14 \mathrm{E}-08$ & $1.14 \mathrm{E}-08$ & $1.46 \mathrm{E}-08$ & $1.15 \mathrm{E}-08$ & $1.24 \mathrm{E}-08$ & $1.24 \mathrm{E}-08$ & $1.23 \mathrm{E}-08$ & $1.24 \mathrm{E}-08$ \\
\hline$\overline{A s}$ & $1.08 \mathrm{E}-06$ & $9.79 \mathrm{E}-07$ & $1.27 \mathrm{E}-06$ & $1.73 \mathrm{E}-06$ & $1.80 \mathrm{E}-05$ & $8.63 \mathrm{E}-06$ & E-05 & $3.13 \mathrm{E}-05$ & $2.25 \mathrm{E}-05$ & $1.19 \mathrm{E}-06$ & $1.48 \mathrm{E}-05$ & $3.75 \mathrm{E}-05$ & $4.11 \mathrm{E}-05$ & $2.26 \mathrm{E}-05$ & $1.56 \mathrm{E}-05$ \\
\hline$\overline{\mathrm{Ba}}$ & 1.07E-09 & $1.07 \mathrm{E}-08$ & $8.02 \mathrm{E}-09$ & $6.97 \mathrm{E}-08$ & ---- & --- & ---- & -- & -- & $2.50 \mathrm{E}-09$ & 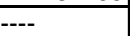 & --- & --- & --- & -- \\
\hline $\mathrm{Ca}$ & $5.05 \mathrm{E}-05$ & $5.04 \mathrm{E}-05$ & $5.05 \mathrm{E}-05$ & $3.15 \mathrm{E}-05$ & $3.09 \mathrm{E}-05$ & 5.15E-05 & $5.06 \mathrm{E}-05$ & $3.09 \mathrm{E}-05$ & $3.09 \mathrm{E}-05$ & $3.17 \mathrm{E}-05$ & $3.09 \mathrm{E}-01$ & 5.04E-05 & $5.04 \mathrm{E}-05$ & 5.03E-05 & $5.08 \mathrm{E}-05$ \\
\hline$\overline{C d}$ & $2.94 \mathrm{E}-08$ & $2.81 \mathrm{E}-08$ & $3.72 \mathrm{E}-08$ & $4.56 \mathrm{E}-08$ & $4.85 \mathrm{E}-07$ & $1.03 \mathrm{E}-07$ & $6.26 \mathrm{E}-07$ & $9.98 \mathrm{E}-07$ & $6.59 \mathrm{E}-07$ & $2.44 \mathrm{E}-08$ & 3.62E-07 & $1.22 \mathrm{E}-06$ & $1.36 \mathrm{E}-06$ & $6.47 \mathrm{E}-07$ & $3.72 \mathrm{E}-07$ \\
\hline$\overline{\mathrm{Cl}}$ & $4.27 \mathrm{E}-02$ & $4.27 \mathrm{E}-02$ & $4.27 \mathrm{E}-02$ & $3.86 \mathrm{E}-02$ & $3.83 \mathrm{E}-02$ & $4.30 \mathrm{E}-02$ & E-02 & $3.84 \mathrm{E}-02$ & $3 \mathrm{E}-02$ & 3.87E-02 & 3.84E-02 & $4.26 \mathrm{E}-02$ & $4.27 \mathrm{E}-02$ & $4.26 \mathrm{E}-02$ & $4.28 \mathrm{E}-02$ \\
\hline Co & $1.57 \mathrm{E}-07$ & $1.60 \mathrm{E}-07$ & 1.32E-07 & $6.91 \mathrm{E}-08$ & $1.03 \mathrm{E}-07$ & $6.64 \mathrm{E}-08$ & E-07 & $8 \mathrm{E}-07$ & $1.21 \mathrm{E}-07$ & $6.66 \mathrm{E}-08$ & $8.95 \mathrm{E}-08$ & 1.86E-07 & $2.00 \mathrm{E}-07$ & $1.24 \mathrm{E}-07$ & $9.49 \mathrm{E}-08$ \\
\hline $\mathrm{Cr}$ & $6.50 \mathrm{E}-06$ & $3.95 \mathrm{E}-06$ & $1.31 \mathrm{E}-05$ & $5.35 \mathrm{E}-05$ & $7.19 \mathrm{E}-05$ & $8.77 \mathrm{E}-05$ & -05 & $6.12 \mathrm{E}-05$ & E-05 & $6.72 \mathrm{E}-05$ & $8 \mathrm{E}-05$ & $6.31 \mathrm{E}-05$ & $3 \mathrm{E}-05$ & $7.51 \mathrm{E}-05$ & $8.15 \mathrm{E}-05$ \\
\hline $\mathrm{Cu}$ & $5.62 \mathrm{E}-05$ & $5.82 \mathrm{E}-05$ & $4.25 \mathrm{E}-05$ & $1.01 \mathrm{E}-05$ & $2.88 \mathrm{E}-05$ & $6.17 \mathrm{E}-06$ & $3.71 \mathrm{E}-05$ & $5.91 \mathrm{E}-05$ & $3.91 \mathrm{E}-05$ & $8.57 \mathrm{E}-06$ & $2.15 \mathrm{E}-05$ & $7.23 \mathrm{E}-05$ & $8.05 \mathrm{E}-05$ & $3.84 \mathrm{E}-05$ & $2.21 \mathrm{E}-05$ \\
\hline$\overline{F e}$ & $4.73 \mathrm{E}-07$ & $4.73 \mathrm{E}-07$ & $3.68 \mathrm{E}-07$ & $2.82 \mathrm{E}-07$ & $1.31 \mathrm{E}-06$ & $3.16 \mathrm{E}-07$ & $1.68 \mathrm{E}-06$ & $2.65 \mathrm{E}-06$ & $1.76 \mathrm{E}-06$ & $6.51 \mathrm{E}-07$ & $9.88 \mathrm{E}-07$ & $3.24 \mathrm{E}-06$ & $3.59 \mathrm{E}-06$ & $1.74 \mathrm{E}-06$ & $1.02 \mathrm{E}-06$ \\
\hline $\bar{F}$ & $7.12 \mathrm{E}-02$ & $7.11 \mathrm{E}-02$ & $7.12 \mathrm{E}-02$ & $3.62 \mathrm{E}-02$ & $\mathrm{E}-02$ & $7.19 \mathrm{E}-02$ & -02 & $0 \mathrm{E}-02$ & E-02 & $1.65 \mathrm{E}+00$ & $1.64 \mathrm{E}+00$ & $3.23 \mathrm{E}+00$ & $3.23 \mathrm{E}+00$ & $3.22 \mathrm{E}+00$ & $3.25 \mathrm{E}+00$ \\
\hline $\mathrm{Hg}$ & $3.66 \mathrm{E}-07$ & $3.66 \mathrm{E}-07$ & $3.66 \mathrm{E}-07$ & $6.82 \mathrm{E}-07$ & $4.16 \mathrm{E}-06$ & $4.32 \mathrm{E}-06$ & $E-06$ & $4.43 \mathrm{E}-06$ & $4.25 \mathrm{E}-06$ & $3.42 \mathrm{E}-07$ & $4.10 \mathrm{E}-06$ & $4.86 \mathrm{E}-06$ & $4.94 \mathrm{E}-06$ & $4.55 \mathrm{E}-06$ & $4.44 \mathrm{E}-06$ \\
\hline $\bar{K}$ & $5.74 \mathrm{E}-03$ & $5.81 \mathrm{E}-03$ & $5.74 \mathrm{E}-03$ & $4.74 \mathrm{E}-03$ & $4.94 \mathrm{E}-03$ & $4.93 \mathrm{E}-03$ & $5.35 \mathrm{E}-03$ & $5.57 \mathrm{E}-03$ & $2.28 \mathrm{E}-01$ & $2.07 \mathrm{E}-01$ & $2.13 \mathrm{E}-01$ & $2.70 \mathrm{E}-01$ & $2.77 \mathrm{E}-01$ & $2.45 \mathrm{E}-01$ & $2.37 \mathrm{E}-01$ \\
\hline $\mathrm{Mg}$ & $-\cdots$ & $-\cdots$ & $3.45 \mathrm{E}-09$ & $4.40 \mathrm{E}-08$ & $1.77 \mathrm{E}-08$ & $5.43 \mathrm{E}-08$ & $\mathrm{E}-08$ & 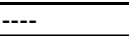 & $3.51 \mathrm{E}-09$ & $4.63 \mathrm{E}-08$ & $2.79 \mathrm{E}-08$ & --- & --- & $9.03 \mathrm{E}-09$ & $3.19 \mathrm{E}-08$ \\
\hline$M n$ & $2.01 \mathrm{E}-08$ & $2.40 \mathrm{E}-08$ & $3.21 \mathrm{E}-08$ & -08 & $6.77 \mathrm{E}-08$ & E-08 & & $1.15 \mathrm{E}-07$ & E-08 & E-08 & $E-08$ & 1.37E-07 & $1.49 \mathrm{E}-07$ & $8.44 \mathrm{E}-08$ & $5 \mathrm{E}-08$ \\
\hline Nd & --- & $-\cdots$ & 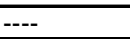 & $1.46 \mathrm{E}-06$ & --- & $1.96 \mathrm{E}-06$ & --. & -.- & --- & $1.59 \mathrm{E}-06$ & $5.41 \mathrm{E}-07$ & -.- & --- & --- & $6.81 \mathrm{E}-07$ \\
\hline$\overline{\mathrm{Ni}}$ & $3.75 \mathrm{E}-07$ & $3.84 \mathrm{E}-07$ & $3.09 \mathrm{E}-07$ & $1.45 \mathrm{E}-07$ & $2.34 \mathrm{E}-07$ & $1.34 \mathrm{E}-07$ & $2.83 \mathrm{E}-07$ & $3.81 \mathrm{E}-07$ & $2.84 \mathrm{E}-07$ & $1.38 \mathrm{E}-07$ & $2.00 \mathrm{E}-07$ & $4.52 \mathrm{E}-07$ & $4.92 \mathrm{E}-07$ & $2.88 \mathrm{E}-07$ & $2.11 \mathrm{E}-07$ \\
\hline$\overline{\mathrm{Pb}}$ & $1.03 \mathrm{E}-06$ & $8.50 \mathrm{E}-07$ & $1.17 \mathrm{E}-06$ & $1.68 \mathrm{E}-06$ & ---- & --- & --- & --- & --- & $9.93 \mathrm{E}-07$ & --- & --- & --- & --- & ---- \\
\hline$\overline{\mathbf{R u}}$ & $5.94 \mathrm{E}-06$ & $6.12 \mathrm{E}-06$ & $4.60 \mathrm{E}-06$ & $1.40 \mathrm{E}-06$ & $3.22 \mathrm{E}-06$ & $1.06 \mathrm{E}-06$ & $4.07 \mathrm{E}-06$ & $6.18 \mathrm{E}-06$ & $4.23 \mathrm{E}-06$ & $1.26 \mathrm{E}-06$ & $2.51 \mathrm{E}-06$ & $7.51 \mathrm{E}-06$ & DE-06 & $4.19 \mathrm{E}-06$ & $2.61 \mathrm{E}-06$ \\
\hline$\overline{\mathrm{Se}}$ & $7.63 \mathrm{E}-06$ & $5.85 \mathrm{E}-06$ & $9.85 \mathrm{E}-06$ & $2.03 \mathrm{E}-05$ & $5.53 \mathrm{E}-05$ & $7.32 \mathrm{E}-05$ & $5.70 \mathrm{E}-05$ & $4.00 \mathrm{E}-05$ & $5.01 \mathrm{E}-05$ & $1.96 \mathrm{E}-05$ & $5.91 \mathrm{E}-05$ & $3.90 \mathrm{E}-05$ & $3.50 \mathrm{E}-05$ & $5.60 \mathrm{E}-05$ & $6.47 \mathrm{E}-05$ \\
\hline$\overline{\mathrm{Si}}$ & $3.64 \mathrm{E}-05$ & $3.19 \mathrm{E}-05$ & $1.86 \mathrm{E}-04$ & $6.09 \mathrm{E}-06$ & -- & --. & ---- & --- & ---- & $4.76 \mathrm{E}-05$ & ---- & --- & $\begin{array}{ll}--- \\
\end{array}$ & ---- & --- \\
\hline $\mathrm{Sr}$ & $\begin{array}{ll}--- \\
\end{array}$ & ---- & $\begin{array}{ll}--- \\
\end{array}$ & $9.83 \mathrm{E}-07$ & --- & $1.36 \mathrm{E}-06$ & --- & --- & --- & $1.08 \mathrm{E}-06$ & $2.71 \mathrm{E}-07$ & --- & --- & --- & 3.67E-07 \\
\hline$Z n$ & $\begin{array}{l}6.83 \mathrm{E}-05 \\
\end{array}$ & $7.04 \mathrm{E}-05$ & \begin{tabular}{|l}
$5.34 \mathrm{E}-05$ \\
\end{tabular} & $1.76 \mathrm{E}-05$ & $3.79 \mathrm{E}-05$ & $1.40 \mathrm{E}-05$ & $4.75 \mathrm{E}-05$ & $7.09 \mathrm{E}-05$ & $4.91 \mathrm{E}-05$ & $1.60 \mathrm{E}-05$ & $3.00 \mathrm{E}-05$ & $8.58 \mathrm{E}-05$ & $9.46 \mathrm{E}-05$ & $4.89 \mathrm{E}-05$ & $3.12 \mathrm{E}-05$ \\
\hline$\overline{Z r}$ & $1.80 \mathrm{E}-06$ & $1.86 \mathrm{E}-06$ & $1.37 \mathrm{E}-06$ & $3.56 \mathrm{E}-07$ & $9.39 \mathrm{E}-07$ & $2.39 \mathrm{E}-07$ & $1.20 \mathrm{E}-06$ & $1.88 \mathrm{E}-06$ & $1.26 \mathrm{E}-06$ & $3.10 \mathrm{E}-07$ & $7.12 \mathrm{E}-07$ & $2.30 \mathrm{E}-06$ & $2.55 \mathrm{E}-06$ & $1.24 \mathrm{E}-06$ & $7.35 \mathrm{E}-07$ \\
\hline Insol.Sol.mg/L & 600 & 600 & 600 & 600 & 600 & 600 & 600 & 600 & 600 & 600 & 600 & 600 & 600 & 600 & 600 \\
\hline
\end{tabular}


Table 12: Projected Feed Composition After Dissolution and Dilution to 6.44 M Total Sodium Concentration $600 \mathrm{mg} / \mathrm{L}$ sludge; $10 \%$ of interstitial supernate remaining (continued)

\begin{tabular}{|c|c|c|c|c|c|c|c|c|c|c|c|c|c|c|c|}
\hline \\
\hline & Tank 25 & Tank 27 & Tank 28 & Tank 29 & Tank 31 & Tank 33 & Tank 34 & Tank 36 & Tank 37 & Tank 38 & Tank 41 & Tank 44 & Tank 45 & Tank 46 & Tank 47 \\
\hline C-14 & 37E-09 & $37 \mathrm{E}-09$ & 3.37E-09 & 3.37E-09 & $3.37 \mathrm{E}-09$ & 3.37E-09 & 3.37E-09 & $3.37 \mathrm{E}-09$ & 3.37E-09 & $3.37 \mathrm{E}-09$ & 3.37E-09 & 3.37E-09 & $3.37 \mathrm{E}-09$ & 3.37E-09 & 3.37E-09 \\
\hline $\mathrm{Ni}-59$ & $1.94 \mathrm{E}-06$ & $1.94 \mathrm{E}-06$ & $1.94 \mathrm{E}-06$ & $1.94 \mathrm{E}-06$ & $1.94 \mathrm{E}-06$ & 1.94E-06 & $1.94 \mathrm{E}-06$ & $1.94 \mathrm{E}-06$ & $1.94 \mathrm{E}-06$ & $1.94 \mathrm{E}-06$ & $1.94 \mathrm{E}-06$ & $1.94 \mathrm{E}-06$ & $1.94 \mathrm{E}-06$ & $1.94 \mathrm{E}-06$ & $1.94 \mathrm{E}-$ \\
\hline Ni-63 & $3.01 \mathrm{E}-09$ & $3.01 \mathrm{E}-09$ & $3.01 \mathrm{E}-09$ & $3.01 \mathrm{E}-09$ & $3.01 \mathrm{E}-09$ & $3.01 \mathrm{E}-09$ & $3.01 \mathrm{E}-09$ & $3.01 \mathrm{E}-09$ & $3.01 \mathrm{E}-09$ & $3.01 \mathrm{E}-09$ & $3.01 \mathrm{E}-09$ & $3.01 \mathrm{E}-09$ & $3.01 \mathrm{E}-09$ & $3.01 \mathrm{E}-09$ & $3.01 \mathrm{E}-0$ \\
\hline 20-60 & $3.09 \mathrm{E}-04$ & $3.09 \mathrm{E}-04$ & $3.09 \mathrm{E}-04$ & $3.09 \mathrm{E}-04$ & $3.09 \mathrm{E}-04$ & & $3.09 \mathrm{E}-04$ & & $3.09 \mathrm{E}-04$ & & $3.09 \mathrm{E}-04$ & $3.09 \mathrm{E}-04$ & $3.09 \mathrm{E}-04$ & $3.09 \mathrm{E}-04$ & \\
\hline Se-79 & & 1.14 & & $1.14 \mathrm{E}-06$ & 14E-06 & & & & 1.14E-06 & $1.14 \mathrm{E}-06$ & $14 \mathrm{E}-\mathrm{C}$ & & & $1.14 \mathrm{E}-06$ & $1.14 \mathrm{E}$ \\
\hline TC-99 & $1.94 \mathrm{E}-05$ & $1.94 \mathrm{E}-05$ & $1.94 \mathrm{E}-05$ & $1.94 \mathrm{E}-05$ & $1.94 \mathrm{E}-05$ & $1.94 \mathrm{E}-05$ & $1.94 \mathrm{E}-05$ & $1.94 \mathrm{E}-05$ & $1.94 \mathrm{E}-05$ & $1.94 \mathrm{E}-05$ & $1.94 \mathrm{E}-05$ & $1.94 \mathrm{E}-05$ & $1.94 \mathrm{E}-05$ & $1.94 \mathrm{E}-05$ & $1.94 \mathrm{E}-$ \\
\hline Ru-106 & $7.18 \mathrm{E}-06$ & $7.18 \mathrm{E}-06$ & $7.18 \mathrm{E}-06$ & $7.18 \mathrm{E}-06$ & $7.18 \mathrm{E}-06$ & $7.18 \mathrm{E}-06$ & $7.18 \mathrm{E}-06$ & $7.18 \mathrm{E}-06$ & $7.18 \mathrm{E}-06$ & $7.18 \mathrm{E}-06$ & $7.18 \mathrm{E}-06$ & $7.18 \mathrm{E}-06$ & $7.18 \mathrm{E}-06$ & $7.18 \mathrm{E}-06$ & $7.18 \mathrm{E}-0$ \\
\hline Rh-106 & $7.18 \mathrm{E}-06$ & $7.18 \mathrm{E}-06$ & $7.18 \mathrm{E}-06$ & $7.18 \mathrm{E}-06$ & $7.18 \mathrm{E}-06$ & $7.18 \mathrm{E}-06$ & & $7.18 \mathrm{E}-06$ & & $7.18 \mathrm{E}-06$ & & $718 \mathrm{E}-06$ & & $7.18 \mathrm{E}-06$ & $7.18 \mathrm{E}-$ \\
\hline Sb-125 & $1.84 \mathrm{E}-04$ & $1.84 \mathrm{E}$ & E-04 & $1.84 \mathrm{E}-04$ & -04 & & & & & $\mathrm{E}-04$ & & & & & \\
\hline Sn-126 & $1.53 \mathrm{E}-06$ & $1.53 \mathrm{E}-06$ & $1.53 \mathrm{E}-06$ & $1.53 \mathrm{E}-06$ & $1.53 \mathrm{E}-06$ & & $3 \overline{\mathrm{E}-06}$ & $1.53 \mathrm{E}-06$ & $1.53 \mathrm{E}-06$ & $1.53 \mathrm{E}-06$ & $1.53 \mathrm{E}-06$ & $1.53 \mathrm{E}-06$ & $1.53 \mathrm{E}-06$ & $1.53 \mathrm{E}-06$ & \\
\hline I-129 & $1.79 \mathrm{E}-10$ & $1.79 \mathrm{E}-10$ & $1.79 \mathrm{E}-10$ & $1.79 \mathrm{E}-10$ & $1.79 \mathrm{E}-10$ & $1.79 \mathrm{E}-10$ & $1.79 \mathrm{E}-10$ & $1.79 \mathrm{E}-10$ & $1.79 \mathrm{E}-10$ & $1.79 \mathrm{E}-10$ & $1.79 \mathrm{E}-10$ & $1.79 \mathrm{E}-10$ & $1.79 \mathrm{E}-10$ & $1.79 \mathrm{E}-10$ & \\
\hline Cs-134 & $8.05 \mathrm{E}-06$ & $8.05 \mathrm{E}-06$ & $8.05 \mathrm{E}-06$ & $8.05 \mathrm{E}-06$ & $8.05 \mathrm{E}-06$ & $8.05 \mathrm{E}-06$ & $8.05 \mathrm{E}-06$ & $8.05 \mathrm{E}-06$ & $8.05 \mathrm{E}-06$ & $8.05 \mathrm{E}-06$ & $8.05 \mathrm{E}-06$ & $8.05 \mathrm{E}-06$ & $8.05 \mathrm{E}-06$ & $8.05 \mathrm{E}-06$ & $8.05 \mathrm{E}-0$ \\
\hline Cs-135 & & $1.37 \mathrm{E}-08$ & & & 1.37E-08 & & & & & $1.37 \mathrm{E}-08$ & & $137 F-08$ & & & \\
\hline Cs-137 & $3.56 \mathrm{E}-02$ & $3.40 \mathrm{E}-02$ & $3.69 \mathrm{E}-02$ & $3.20 \mathrm{E}-02$ & $8.11 \mathrm{E}-02$ & $1 \mathrm{E}-03$ & 2.97E-02 & $1.46 \mathrm{E}-01$ & $1.07 \mathrm{E}-01$ & $5.93 \mathrm{E}-03$ & $2.12 \mathrm{E}-02$ & $4.29 \mathrm{E}-02$ & $4.08 \mathrm{E}-02$ & $5.14 \mathrm{E}-02$ & $2.37 \mathrm{E}$ \\
\hline Ba-137m & $3.37 \mathrm{E}-02$ & $3.21 \mathrm{E}-02$ & $3.49 \mathrm{E}-02$ & $3.03 \mathrm{E}-02$ & $7.67 \mathrm{E}-02$ & $4.55 \mathrm{E}-03$ & $1 \mathrm{E}-02$ & $1.38 \mathrm{E}-01$ & $1.01 \mathrm{E}-01$ & $5.61 \mathrm{E}-03$ & $2.01 \mathrm{E}-02$ & $4.06 \mathrm{E}-02$ & $3.86 \mathrm{E}-02$ & 4.87E-02 & $2.25 \mathrm{E}$ \\
\hline Sr-90 & $6.73 \mathrm{E}-02$ & $6.73 \mathrm{E}-02$ & $6.73 \mathrm{E}-02$ & $6.74 \mathrm{E}-02$ & $6.74 \mathrm{E}-02$ & $6.74 \mathrm{E}-02$ & & $6.74 \mathrm{E}-02$ & $6.74 \mathrm{E}-02$ & $6.73 \mathrm{E}-02$ & $6.73 \mathrm{E}-02$ & $6.73 \mathrm{E}-02$ & $6.73 \mathrm{E}-02$ & $6.73 \mathrm{E}-02$ & $6.73 \mathrm{E}-\mathrm{C}$ \\
\hline $\mathrm{Y}-90$ & $6.73 \mathrm{E}-02$ & $6.73 \mathrm{E}-02$ & $6.73 \mathrm{E}-02$ & $6.74 \mathrm{E}-02$ & $6.74 \mathrm{E}-02$ & $6.74 \mathrm{E}-02$ & $6.74 \mathrm{E}-02$ & $6.74 \mathrm{E}-02$ & $6.74 \mathrm{E}-02$ & $6.73 \mathrm{E}-02$ & $6.73 \mathrm{E}-02$ & $6.73 \mathrm{E}-02$ & $6.73 \mathrm{E}-02$ & $6.73 \mathrm{E}-02$ & $6.73 \mathrm{E}-\mathrm{C}$ \\
\hline $\mathrm{H}-3$ & & & & & & & & & & & & & & & \\
\hline Ce-144 & $3.70 \mathrm{E}-06$ & $3.70 \mathrm{E}-06$ & $3.70 \mathrm{E}-06$ & $3.70 \mathrm{E}-06$ & $3.70 \mathrm{E}-06$ & $3.70 \mathrm{E}-06$ & $3.70 \mathrm{E}-06$ & $3.70 \mathrm{E}-06$ & $3.70 \mathrm{E}-06$ & $3.70 \mathrm{E}-06$ & $3.70 \mathrm{E}-06$ & $3.70 \mathrm{E}-06$ & $3.70 \mathrm{E}-06$ & $3.70 \mathrm{E}-06$ & $3.70 \mathrm{E}$ \\
\hline Pr-144 & $3.70 \mathrm{E}-06$ & $3.70 \mathrm{E}-06$ & $3.70 \mathrm{E}-06$ & $3.70 \mathrm{E}-06$ & $3.70 \mathrm{E}-06$ & -06 & & $3.70 \mathrm{E}-06$ & E-06 & $3.70 \mathrm{E}-06$ & $\mathrm{E}-06$ & 3.7 & E-06 & $3.70 \mathrm{E}-06$ & $O E^{-}$ \\
\hline$n-1$ & & 3.88 & & $3.88 \mathrm{E}-03$ & & & & & & & & & & & \\
\hline Eu-154 & 7.56 & $7.56 \mathrm{E}-04$ & $7.56 \mathrm{E}-04$ & $7.56 \mathrm{E}-04$ & $7.56 \mathrm{E}-04$ & & & $7.56 \mathrm{E}-04$ & 7.5 & $7.56 \mathrm{E}-04$ & $E-04$ & 7.5 & & $6 \mathrm{E}-04$ & 56 \\
\hline Th-232 & 2.17 & $2.17 \mathrm{E}-09$ & 17E-09 & $2.17 \mathrm{E}-09$ & $2.17 \mathrm{E}-09$ & & & $2.17 \mathrm{E}-09$ & $2.17 \mathrm{E}-09$ & $2.17 \mathrm{E}-09$ & & & & $2.17 \mathrm{E}-09$ & \\
\hline $\mathrm{U}-232$ & 4.94 & 4.9 & & $4.69 \mathrm{E}-10$ & & & & & & & & & & & \\
\hline 233 & 7.35 & 7.35 & & $7.42 \mathrm{E}-08$ & $7.42 \mathrm{E}-08$ & & & $7.42 \mathrm{E}-08$ & & $7.35 \mathrm{E}-08$ & & 7.3 & & $7.35 \mathrm{E}-08$ & \\
\hline & & 2.35 & & $2.36 \mathrm{E}-08$ & 2.3 & & & 2.36 & & & & & & & \\
\hline 235 & & -09 & & $1.16 \mathrm{E}$ & 1. & & & $1.16 \mathrm{E}-0$ & & 1.18 & & & & $1.22 \mathrm{E}-09$ & $1.22 \mathrm{E}$ \\
\hline U-236 & & & & $3.35 \mathrm{E}-09$ & & & & & & & & & & $4 \mathrm{E}-09$ & \\
\hline 238 & 4.09E-08 & $\begin{array}{l}4.08 \mathrm{E}-08 \\
\end{array}$ & & $3.57 \mathrm{E}-08$ & $3.57 \mathrm{E}-08$ & & & $3.57 \mathrm{E}-08$ & $3.57 \mathrm{E}-08$ & $3.56 \mathrm{E}-08$ & & & & $8 \mathrm{E}-08$ & \\
\hline-23 & & 5. & & 5.11 & & & & & & & & & & & \\
\hline $1-23$ & & 1. & & & & & & & & & & & & & \\
\hline Pu-239 & & & & & & & & $2.46 \mathrm{E}-05$ & & & & & & $3.08 \mathrm{E}-05$ & \\
\hline Pu-240 & $1.28 \mathrm{E}-05$ & $1.28 \mathrm{E}-05$ & $1.28 \mathrm{E}-05$ & $1.14 \mathrm{E}-05$ & $1.14 \mathrm{E}-05$ & & & $1.14 \mathrm{E}-05$ & & $1.15 \mathrm{E}-05$ & $1.15 \mathrm{E}-05$ & & & $1.28 \mathrm{E}-05$ & \\
\hline Pu-241 & 8.17 & 8.17E-04 & 8.17 & 7.80 & $7.80 \mathrm{E}-04$ & & & $\mathrm{E}-04$ & & & & & & & \\
\hline $1-24$ & & & & & & & & 2.23 & & & & & & & \\
\hline Am-2 & & $E-04$ & & $1.62 \mathrm{E}-04$ & $E-04$ & & & $1.62 \mathrm{E}-04$ & 1.6 & & & & & 1.62E-04 & \\
\hline$A_{r}$ & $1.52 \mathrm{E}-07$ & $1.52 \mathrm{E}-07$ & $1.52 \mathrm{E}-07$ & $1.52 \mathrm{E}-07$ & $1.52 \mathrm{E}-07$ & $2.15 \mathrm{E}-07$ & $2.16 \mathrm{E}-07$ & $1.52 \mathrm{E}-07$ & $1.52 \mathrm{E}-07$ & $1.52 \mathrm{E}-07$ & $1.52 \mathrm{E}-07$ & $1.52 \mathrm{E}-07$ & $1.52 \mathrm{E}-07$ & $1.52 \mathrm{E}-07$ & $1.52 \mathrm{E}-0$ \\
\hline & & & & & & & & & & & & & & & \\
\hline & & & & & & & & & & & & & & & \\
\hline $\mathrm{Cm}-245$ & $1.24 \mathrm{E}-08$ & 1.24 & $1.24 \mathrm{E}-08$ & $1.24 \mathrm{E}-08$ & 1.24 & & & $1.24 \mathrm{E}-08$ & & & & 1.2 & & $1.24 \mathrm{E}-08$ & \\
\hline Total Alpha & $1.85 \mathrm{E}-03$ & $1.85 \mathrm{E}-03$ & $1.85 \mathrm{E}-03$ & $1.80 \mathrm{E}-03$ & $1.80 \mathrm{E}-03$ & $1.85 \mathrm{E}-03$ & $1.85 \mathrm{E}-03$ & $1.80 \mathrm{E}-03$ & $1.80 \mathrm{E}-03$ & $1.84 \mathrm{E}-03$ & $1.84 \mathrm{E}-03$ & $1.85 \mathrm{E}-03$ & $1.85 \mathrm{E}-03$ & $1.85 \mathrm{E}-03$ & $1.85 \mathrm{E}-0$ \\
\hline
\end{tabular}


Table 13: Projected Feed Composition After Dissolution and Dilution to 6.44 M Total Sodium Concentration $350 \mathrm{mg} / \mathrm{L}$ sludge; $10 \%$ of interstitial supernate remaining

\begin{tabular}{|c|c|c|c|c|c|c|c|c|c|c|c|c|c|c|c|}
\hline \multicolumn{16}{|c|}{ Concentration (M) } \\
\hline & Tank 25 & Tank 27 & Tank 28 & Tank 29 & Tank 31 & Tank 33 & Tank 34 & Tank 36 & Tank 37 & Tank 38 & Tank 41 & Tank 44 & Tank 45 & Tank 46 & Tank 47 \\
\hline $\mathrm{Na}$ & $6.44 \mathrm{E}+00$ & $6.44 \mathrm{E}+00$ & $6.44 \mathrm{E}+00$ & $6.44 \mathrm{E}+00$ & $6.44 \mathrm{E}+00$ & $6.44 \mathrm{E}+00$ & $6.44 \mathrm{E}+00$ & $6.44 \mathrm{E}+00$ & $6.44 \mathrm{E}+00$ & $6.44 \mathrm{E}+00$ & $6.44 \mathrm{E}+00$ & $6.44 \mathrm{E}+00$ & $6.44 \mathrm{E}+00$ & $6.44 \mathrm{E}+00$ & $6.44 \mathrm{E}+00$ \\
\hline $\mathrm{Al}(\mathrm{OH}) 4$ & $3.22 \mathrm{E}-01$ & $3.25 \mathrm{E}-01$ & $3.22 \mathrm{E}-01$ & $3.99 \mathrm{E}-01$ & $4.00 \mathrm{E}-01$ & $3.21 \mathrm{E}-01$ & $3.20 \mathrm{E}-01$ & $3.94 \mathrm{E}-01$ & 3.97E-01 & $4.00 \mathrm{E}-01$ & 3.96E-01 & $3.20 \mathrm{E}-01$ & $3.19 \mathrm{E}-01$ & $3.17 \mathrm{E}-01$ & $3.22 \mathrm{E}-01$ \\
\hline $\mathrm{CO} 3$ & $2.22 \mathrm{E}-01$ & $2.22 \mathrm{E}-01$ & $2.21 \mathrm{E}-01$ & $1.33 \mathrm{E}-01$ & $1.32 \mathrm{E}-01$ & $2.23 \mathrm{E}-01$ & $2.25 \mathrm{E}-01$ & $1.33 \mathrm{E}-01$ & $1.32 \mathrm{E}-01$ & $1.34 \mathrm{E}-01$ & 1.34E-01 & $2.22 \mathrm{E}-01$ & $2.23 \mathrm{E}-01$ & $2.20 \mathrm{E}-01$ & $2.24 \mathrm{E}-01$ \\
\hline$\overline{\mathrm{C2O}}$ & $2.23 \mathrm{E}-02$ & $2.23 \mathrm{E}-02$ & $2.23 \mathrm{E}-02$ & $1.42 \mathrm{E}-02$ & $1.41 \mathrm{E}-02$ & $2.25 \mathrm{E}-02$ & $2.23 \mathrm{E}-02$ & $1.41 \mathrm{E}-02$ & $1.41 \mathrm{E}-02$ & $1.42 \mathrm{E}-02$ & $1.41 \mathrm{E}-02$ & $2.22 \mathrm{E}-02$ & $2.23 \mathrm{E}-02$ & $2.22 \mathrm{E}-02$ & $2.24 \mathrm{E}-02$ \\
\hline NO2 & $1.11 \mathrm{E}-01$ & $1.11 \mathrm{E}-01$ & $1.14 \mathrm{E}-01$ & $7.44 \mathrm{E}-01$ & $7.49 \mathrm{E}-01$ & $1.02 \mathrm{E}-01$ & $1.10 \mathrm{E}-01$ & 7.47E-01 & $7.45 \mathrm{E}-01$ & $7.43 \mathrm{E}-01$ & $7.41 \mathrm{E}-01$ & $1.12 \mathrm{E}-01$ & $1.13 \mathrm{E}-01$ & $1.12 \mathrm{E}-01$ & $1.06 \mathrm{E}-01$ \\
\hline$\overline{\mathrm{NO} 3}$ & $3.80 \mathrm{E}+00$ & $3.80 \mathrm{E}+00$ & $3.80 \mathrm{E}+00$ & $2.66 \mathrm{E}+00$ & $2.65 \mathrm{E}+00$ & $3.84 \mathrm{E}+00$ & $3.81 \mathrm{E}+00$ & $2.65 \mathrm{E}+00$ & $2.65 \mathrm{E}+00$ & $2.67 \mathrm{E}+00$ & $2.67 \mathrm{E}+00$ & $3.79 \mathrm{E}+00$ & $3.79 \mathrm{E}+00$ & $3.79 \mathrm{E}+00$ & $3.82 \mathrm{E}+00$ \\
\hline$\overline{\mathrm{OH}}$ & $9.63 \mathrm{E}-01$ & $9.65 \mathrm{E}-01$ & $9.47 \mathrm{E}-01$ & $1.85 \mathrm{E}+00$ & $1.86 \mathrm{E}+00$ & $9.14 \mathrm{E}-01$ & & $1.89 \mathrm{E}+00$ & $1.87 \mathrm{E}+00$ & $1.85 \mathrm{E}+00$ & $1.85 \mathrm{E}+00$ & $9.80 \mathrm{E}-01$ & $9.90 \mathrm{E}-01$ & $9.39 \mathrm{E}-01$ & $9.27 \mathrm{E}-01$ \\
\hline PO4 & $1.42 \mathrm{E}-03$ & $1.42 \mathrm{E}-03$ & $1.46 \mathrm{E}-03$ & $1.57 \mathrm{E}-02$ & 1.57E-02 & $1.34 \mathrm{E}-03$ & $1.34 \mathrm{E}-03$ & $1.57 \mathrm{E}-02$ & $1.56 \mathrm{E}-02$ & $1.57 \mathrm{E}-02$ & $1.57 \mathrm{E}-02$ & $1.37 \mathrm{E}-03$ & $1.38 \mathrm{E}-03$ & $1.41 \mathrm{E}-03$ & $1.34 \mathrm{E}-03$ \\
\hline SO4 & $3.25 \mathrm{E}-01$ & $3.25 \mathrm{E}-01$ & $3.25 \mathrm{E}-01$ & $1.84 \mathrm{E}-01$ & $1.83 \mathrm{E}-01$ & $3.29 \mathrm{E}-01$ & $3.26 \mathrm{E}-01$ & $1.83 \mathrm{E}-01$ & $1.83 \mathrm{E}-01$ & $1.85 \mathrm{E}-01$ & $1.83 \mathrm{E}-01$ & $3.24 \mathrm{E}-01$ & $3.25 \mathrm{E}-01$ & $3.24 \mathrm{E}-01$ & $3.27 \mathrm{E}-01$ \\
\hline$\overline{\mathrm{Ag}}$ & $6.13 \mathrm{E}-09$ & $3.60 \mathrm{E}-08$ & $2.65 \mathrm{E}-08$ & $1.33 \mathrm{E}-08$ & $1.14 \mathrm{E}-08$ & $1.25 \mathrm{E}-08$ & $1.24 \mathrm{E}-08$ & $1.14 \mathrm{E}-08$ & $1.14 \mathrm{E}-08$ & $1.46 \mathrm{E}-08$ & $1.15 \mathrm{E}-08$ & $1.24 \mathrm{E}-08$ & $1.24 \mathrm{E}-08$ & $1.23 \mathrm{E}-08$ & $1.24 \mathrm{E}-08$ \\
\hline$\overline{A s}$ & $1.08 \mathrm{E}-06$ & $9.79 \mathrm{E}-07$ & $1.27 \mathrm{E}-06$ & $1.73 \mathrm{E}-06$ & $1.80 \mathrm{E}-05$ & $8.63 \mathrm{E}-06$ & E-05 & $3.13 \mathrm{E}-05$ & $2.25 \mathrm{E}-05$ & $9 \mathrm{E}-06$ & $1.48 \mathrm{E}-05$ & $3.75 \mathrm{E}-05$ & $4.11 \mathrm{E}-05$ & $2.26 \mathrm{E}-05$ & $1.56 \mathrm{E}-05$ \\
\hline$\overline{\mathrm{Ba}}$ & 1.07E-09 & $1.07 \mathrm{E}-08$ & $8.02 \mathrm{E}-09$ & $6.97 \mathrm{E}-08$ & ---- & --- & ---- & -- & -- & $2.50 \mathrm{E}-09$ & 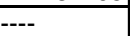 & --- & --- & --- & --- \\
\hline $\mathrm{Ca}$ & $5.05 \mathrm{E}-05$ & $5.04 \mathrm{E}-05$ & $5.05 \mathrm{E}-05$ & $3.15 \mathrm{E}-05$ & $3.09 \mathrm{E}-05$ & 5.15E-05 & $5.06 \mathrm{E}-05$ & $3.09 \mathrm{E}-05$ & $3.09 \mathrm{E}-05$ & $3.17 \mathrm{E}-05$ & $3.09 \mathrm{E}-01$ & 5.04E-05 & $5.04 \mathrm{E}-05$ & 5.03E-05 & $5.08 \mathrm{E}-05$ \\
\hline$\overline{C d}$ & $2.94 \mathrm{E}-08$ & $2.81 \mathrm{E}-08$ & $3.72 \mathrm{E}-08$ & $4.56 \mathrm{E}-08$ & $4.85 \mathrm{E}-07$ & $1.03 \mathrm{E}-07$ & $6.26 \mathrm{E}-07$ & $9.98 \mathrm{E}-07$ & $6.59 \mathrm{E}-07$ & $2.44 \mathrm{E}-08$ & 3.62E-07 & $1.22 \mathrm{E}-06$ & $1.36 \mathrm{E}-06$ & $6.47 \mathrm{E}-07$ & $3.72 \mathrm{E}-07$ \\
\hline$\overline{\mathrm{Cl}}$ & $4.27 \mathrm{E}-02$ & $4.27 \mathrm{E}-02$ & $4.27 \mathrm{E}-02$ & $3.86 \mathrm{E}-02$ & $3.83 \mathrm{E}-02$ & $4.30 \mathrm{E}-02$ & E-02 & $3.84 \mathrm{E}-02$ & E-02 & 3.87E-02 & 3.84E-02 & $4.26 \mathrm{E}-02$ & $4.27 \mathrm{E}-02$ & $4.26 \mathrm{E}-02$ & $4.28 \mathrm{E}-02$ \\
\hline Co & $1.57 \mathrm{E}-07$ & $1.60 \mathrm{E}-07$ & 1.32E-07 & $6.91 \mathrm{E}-08$ & $1.03 \mathrm{E}-07$ & $6.64 \mathrm{E}-08$ & E-07 & $8 \mathrm{E}-07$ & $1.21 \mathrm{E}-07$ & $6.66 \mathrm{E}-08$ & $8.95 \mathrm{E}-08$ & $1.86 \mathrm{E}-07$ & $2.00 \mathrm{E}-07$ & $1.24 \mathrm{E}-07$ & $9.49 \mathrm{E}-08$ \\
\hline $\mathrm{Cr}$ & $6.50 \mathrm{E}-06$ & $3.95 \mathrm{E}-06$ & $1.31 \mathrm{E}-05$ & $5.35 \mathrm{E}-05$ & $7.19 \mathrm{E}-05$ & $8.77 \mathrm{E}-05$ & -05 & $6.12 \mathrm{E}-05$ & $3 \mathrm{E}-05$ & $6.72 \mathrm{E}-05$ & $8 \mathrm{E}-05$ & $6.31 \mathrm{E}-05$ & $3 \mathrm{E}-05$ & $7.51 \mathrm{E}-05$ & $8.15 \mathrm{E}-05$ \\
\hline $\mathrm{Cu}$ & $5.62 \mathrm{E}-05$ & $5.82 \mathrm{E}-05$ & $4.25 \mathrm{E}-05$ & $1.01 \mathrm{E}-05$ & $2.88 \mathrm{E}-05$ & $6.17 \mathrm{E}-06$ & $3.71 \mathrm{E}-05$ & $5.91 \mathrm{E}-05$ & $3.91 \mathrm{E}-05$ & $8.57 \mathrm{E}-06$ & $2.15 \mathrm{E}-05$ & $7.23 \mathrm{E}-05$ & $8.05 \mathrm{E}-05$ & $3.84 \mathrm{E}-05$ & $2.21 \mathrm{E}-05$ \\
\hline$\overline{F e}$ & $4.73 \mathrm{E}-07$ & $4.73 \mathrm{E}-07$ & $3.68 \mathrm{E}-07$ & $2.82 \mathrm{E}-07$ & $1.31 \mathrm{E}-06$ & $3.16 \mathrm{E}-07$ & $1.68 \mathrm{E}-06$ & $2.65 \mathrm{E}-06$ & $1.76 \mathrm{E}-06$ & $6.51 \mathrm{E}-07$ & $9.88 \mathrm{E}-07$ & $3.24 \mathrm{E}-06$ & $3.59 \mathrm{E}-06$ & $1.74 \mathrm{E}-06$ & $1.02 \mathrm{E}-06$ \\
\hline $\bar{F}$ & $7.12 \mathrm{E}-02$ & $7.11 \mathrm{E}-02$ & $7.12 \mathrm{E}-02$ & $3.62 \mathrm{E}-02$ & $\mathrm{E}-02$ & $7.19 \mathrm{E}-02$ & -02 & $0 \mathrm{E}-02$ & $\mathrm{E}-02$ & $3.63 \mathrm{E}-02$ & DE-02 & $7.11 \mathrm{E}-02$ & $7.11 \mathrm{E}-02$ & $7.09 \mathrm{E}-02$ & $7.15 \mathrm{E}-02$ \\
\hline $\mathrm{Hg}$ & $3.66 \mathrm{E}-07$ & $3.66 \mathrm{E}-07$ & $3.66 \mathrm{E}-07$ & $6.82 \mathrm{E}-07$ & $4.16 \mathrm{E}-06$ & $4.32 \mathrm{E}-06$ & $\mathrm{E}-06$ & $4.43 \mathrm{E}-06$ & $4.25 \mathrm{E}-06$ & $3.42 \mathrm{E}-07$ & $4.10 \mathrm{E}-06$ & $4.86 \mathrm{E}-06$ & $4.94 \mathrm{E}-06$ & $4.55 \mathrm{E}-06$ & 4.44E-06 \\
\hline $\bar{K}$ & $5.74 \mathrm{E}-03$ & $5.81 \mathrm{E}-03$ & $5.74 \mathrm{E}-03$ & $4.74 \mathrm{E}-03$ & $4.94 \mathrm{E}-03$ & $4.93 \mathrm{E}-03$ & $5.35 \mathrm{E}-03$ & $5.57 \mathrm{E}-03$ & $5.01 \mathrm{E}-03$ & $4.56 \mathrm{E}-03$ & $4.68 \mathrm{E}-03$ & $5.95 \mathrm{E}-03$ & $6.10 \mathrm{E}-03$ & $5.40 \mathrm{E}-03$ & $5.20 \mathrm{E}-03$ \\
\hline $\mathrm{Mg}$ & $-\cdots$ & $-\cdots$ & $3.45 \mathrm{E}-09$ & $4.40 \mathrm{E}-08$ & $1.77 \mathrm{E}-08$ & $5.43 \mathrm{E}-08$ & $\mathrm{E}-08$ & 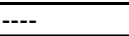 & $3.51 \mathrm{E}-09$ & $4.63 \mathrm{E}-08$ & $2.79 \mathrm{E}-08$ & 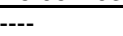 & --- & $9.03 \mathrm{E}-09$ & $3.19 \mathrm{E}-08$ \\
\hline$M n$ & $2.01 \mathrm{E}-08$ & $2.40 \mathrm{E}-08$ & $3.21 \mathrm{E}-08$ & E-08 & $6.77 \mathrm{E}-08$ & E-08 & & $1.15 \mathrm{E}-07$ & $\mathrm{E}-08$ & E-08 & $\mathrm{E}-08$ & $1.37 \mathrm{E}-07$ & $1.49 \mathrm{E}-07$ & $8.44 \mathrm{E}-08$ & $5 \mathrm{E}-08$ \\
\hline Nd & --- & $-\cdots$ & 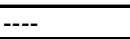 & $1.46 \mathrm{E}-06$ & --- & $1.96 \mathrm{E}-06$ & --. & -.- & --- & $1.59 \mathrm{E}-06$ & $5.41 \mathrm{E}-07$ & --- & --- & --- & $6.81 \mathrm{E}-07$ \\
\hline$\overline{\mathrm{Ni}}$ & $3.75 \mathrm{E}-07$ & $3.84 \mathrm{E}-07$ & $3.09 \mathrm{E}-07$ & $1.45 \mathrm{E}-07$ & $2.34 \mathrm{E}-07$ & $1.34 \mathrm{E}-07$ & $2.83 \mathrm{E}-07$ & $3.81 \mathrm{E}-07$ & $2.84 \mathrm{E}-07$ & $1.38 \mathrm{E}-07$ & $2.00 \mathrm{E}-07$ & $4.52 \mathrm{E}-07$ & $4.92 \mathrm{E}-07$ & $2.88 \mathrm{E}-07$ & $2.11 \mathrm{E}-07$ \\
\hline$\overline{\mathrm{Pb}}$ & $1.03 \mathrm{E}-06$ & $8.50 \mathrm{E}-07$ & $1.17 \mathrm{E}-06$ & $1.68 \mathrm{E}-06$ & ---- & --- & --- & --- & --- & $9.93 \mathrm{E}-07$ & --- & --- & --- & --- & --- \\
\hline$\overline{\mathbf{R u}}$ & $5.94 \mathrm{E}-06$ & $6.12 \mathrm{E}-06$ & $4.60 \mathrm{E}-06$ & $1.40 \mathrm{E}-06$ & $3.22 \mathrm{E}-06$ & $1.06 \mathrm{E}-06$ & $4.07 \mathrm{E}-06$ & $6.18 \mathrm{E}-06$ & $4.23 \mathrm{E}-06$ & $1.26 \mathrm{E}-06$ & $2.51 \mathrm{E}-06$ & $7.51 \mathrm{E}-06$ & $\mathrm{E}-06$ & $4.19 \mathrm{E}-06$ & $2.61 \mathrm{E}-06$ \\
\hline$\overline{\mathrm{Se}}$ & $7.63 \mathrm{E}-06$ & $5.85 \mathrm{E}-06$ & $9.85 \mathrm{E}-06$ & $2.03 \mathrm{E}-05$ & $5.53 \mathrm{E}-05$ & $7.32 \mathrm{E}-05$ & $5.70 \mathrm{E}-05$ & $4.00 \mathrm{E}-05$ & $5.01 \mathrm{E}-05$ & $1.96 \mathrm{E}-05$ & $5.91 \mathrm{E}-05$ & $3.90 \mathrm{E}-05$ & $3.50 \mathrm{E}-05$ & $5.60 \mathrm{E}-05$ & $6.47 \mathrm{E}-05$ \\
\hline$\overline{\mathrm{Si}}$ & $3.64 \mathrm{E}-05$ & $3.19 \mathrm{E}-05$ & $1.86 \mathrm{E}-04$ & $6.09 \mathrm{E}-06$ & -- & --. & ---- & --- & ---- & $4.76 \mathrm{E}-05$ & ---- & --- & $\begin{array}{ll}--- \\
\end{array}$ & ---- & --- \\
\hline $\mathrm{Sr}$ & $\begin{array}{ll}--- \\
\end{array}$ & ---- & $\begin{array}{ll}--- \\
\end{array}$ & $9.83 \mathrm{E}-07$ & --- & $1.36 \mathrm{E}-06$ & --- & --- & ---- & $1.08 \mathrm{E}-06$ & $2.71 \mathrm{E}-07$ & --- & --- & --- & 3.67E-07 \\
\hline$Z n$ & $\begin{array}{l}6.83 \mathrm{E}-05 \\
\end{array}$ & $7.04 \mathrm{E}-05$ & \begin{tabular}{|l}
$5.34 \mathrm{E}-05$ \\
\end{tabular} & $1.76 \mathrm{E}-05$ & $3.79 \mathrm{E}-05$ & $1.40 \mathrm{E}-05$ & $4.75 \mathrm{E}-05$ & $7.09 \mathrm{E}-05$ & $4.91 \mathrm{E}-05$ & $1.60 \mathrm{E}-05$ & $3.00 \mathrm{E}-05$ & $8.58 \mathrm{E}-05$ & $9.46 \mathrm{E}-05$ & $4.89 \mathrm{E}-05$ & $3.12 \mathrm{E}-05$ \\
\hline$\overline{Z r}$ & $1.80 \mathrm{E}-06$ & $1.86 \mathrm{E}-06$ & $1.37 \mathrm{E}-06$ & $3.56 \mathrm{E}-07$ & $9.39 \mathrm{E}-07$ & $2.39 \mathrm{E}-07$ & $1.20 \mathrm{E}-06$ & $1.88 \mathrm{E}-06$ & $1.26 \mathrm{E}-06$ & $3.10 \mathrm{E}-07$ & $7.12 \mathrm{E}-07$ & $2.30 \mathrm{E}-06$ & $2.55 \mathrm{E}-06$ & $1.24 \mathrm{E}-06$ & $7.35 \mathrm{E}-07$ \\
\hline Insol.Sol.mg/L & 350 & 350 & 350 & 350 & 350 & 350 & 350 & 350 & 350 & 350 & 350 & 350 & 350 & 350 & 350 \\
\hline
\end{tabular}


Table 13: Projected Feed Composition After Dissolution and Dilution to 6.44 M Total Sodium Concentration $350 \mathrm{mg} / \mathrm{L}$ sludge; $10 \%$ of interstitial supernate remaining (continued)

\begin{tabular}{|c|c|c|c|c|c|c|c|c|c|c|c|c|c|c|c|}
\hline \multicolumn{16}{|c|}{ clic } \\
\hline & Tank 25 & Tank 27 & Tank 28 & Tank 29 & Tank 31 & Tank 33 & Tank 34 & Tank 36 & Tank 37 & Tank 38 & Tank 41 & Tank 44 & Tank 45 & Tank 46 & Tank 47 \\
\hline C-14 & $1.97 \mathrm{E}-09$ & $1.97 \mathrm{E}-09$ & $1.97 \mathrm{E}-09$ & $1.97 \mathrm{E}-09$ & $1.97 \mathrm{E}-09$ & $1.97 \mathrm{E}-09$ & $1.97 \mathrm{E}-09$ & $1.97 \mathrm{E}-09$ & $1.97 \mathrm{E}-09$ & $1.97 \mathrm{E}-09$ & 1.97E-09 & 1.97E-09 & $1.97 \mathrm{E}-09$ & 1.97E-09 & $1.97 \mathrm{E}-09$ \\
\hline Ni-59 & $1.13 \mathrm{E}-06$ & $1.13 \mathrm{E}-06$ & $1.13 \mathrm{E}-06$ & $1.13 \mathrm{E}-06$ & $1.13 \mathrm{E}-06$ & $1.13 \mathrm{E}-06$ & $1.13 \mathrm{E}-06$ & $1.13 \mathrm{E}-06$ & $1.13 \mathrm{E}-06$ & $1.13 \mathrm{E}-06$ & $1.13 \mathrm{E}-06$ & $1.13 \mathrm{E}-06$ & $1.13 \mathrm{E}-06$ & $1.13 \mathrm{E}-06$ & $1.13 \mathrm{E}-06$ \\
\hline Ni-63 & $1.76 \mathrm{E}-09$ & $1.76 \mathrm{E}-09$ & $1.76 \mathrm{E}-09$ & $1.76 \mathrm{E}-09$ & $1.76 \mathrm{E}-09$ & $1.76 \mathrm{E}-09$ & $1.76 \mathrm{E}-09$ & $1.76 \mathrm{E}-09$ & $1.76 \mathrm{E}-09$ & $1.76 \mathrm{E}-09$ & $1.76 \mathrm{E}-09$ & $1.76 \mathrm{E}-09$ & $1.76 \mathrm{E}-09$ & $1.76 \mathrm{E}-09$ & $1.76 \mathrm{E}-09$ \\
\hline Co-60 & $1.80 \mathrm{E}-04$ & $1.80 \mathrm{E}-04$ & $1.80 \mathrm{E}-04$ & $1.80 \mathrm{E}-04$ & $1.80 \mathrm{E}-04$ & $1.80 \mathrm{E}-04$ & $1.80 \mathrm{E}-04$ & $1.80 \mathrm{E}-04$ & $1.80 \mathrm{E}-04$ & $1.80 \mathrm{E}-04$ & $1.80 \mathrm{E}-04$ & $1.80 \mathrm{E}-04$ & $80 \mathrm{E}-04$ & $1.80 \mathrm{E}-04$ & $1.80 \mathrm{E}-04$ \\
\hline Se-79 & & $6.67 \mathrm{E}-07$ & & & & $6.67 \mathrm{E}-07$ & & & & & & & & $6.67 \mathrm{E}-07$ & \\
\hline Tc-99 & $13 \mathrm{E}-05$ & $1.13 \mathrm{E}-05$ & $1.13 \mathrm{E}-05$ & $1.13 \mathrm{E}-05$ & $1.13 \mathrm{E}-05$ & $1.13 \mathrm{E}-05$ & $1.13 \mathrm{E}-05$ & $1.13 \mathrm{E}-05$ & $1.13 \mathrm{E}-05$ & $1.13 \mathrm{E}-05$ & $1.13 \mathrm{E}-05$ & $1.13 \mathrm{E}-05$ & $1.13 \mathrm{E}-05$ & $1.13 \mathrm{E}-05$ & $1.13 \mathrm{E}-05$ \\
\hline Ru-106 & $4.19 \mathrm{E}-06$ & $4.19 \mathrm{E}-06$ & $4.19 \mathrm{E}-06$ & $4.19 \mathrm{E}-06$ & $4.19 \mathrm{E}-06$ & $4.19 \mathrm{E}-06$ & $4.19 \mathrm{E}-06$ & $4.19 \mathrm{E}-06$ & 4.19E-06 & $4.19 \mathrm{E}-06$ & $4.19 \mathrm{E}-06$ & 4.19E-06 & $4.19 \mathrm{E}-06$ & $4.19 \mathrm{E}-06$ & $4.19 \mathrm{E}-06$ \\
\hline Rh-106 & $4.19 \mathrm{E}-06$ & $4.19 \mathrm{E}-06$ & $4.19 \mathrm{E}-06$ & $4.19 \mathrm{E}-06$ & $4.19 \mathrm{E}-06$ & $4.19 \mathrm{E}-06$ & $4.19 \mathrm{E}-06$ & $4.19 \mathrm{E}-06$ & $4.19 \mathrm{E}-06$ & $4.19 \mathrm{E}-06$ & $4.19 \mathrm{E}-06$ & $4.19 \mathrm{E}-06$ & & $4.19 \mathrm{E}-06$ & $419 \mathrm{~F}-0$ \\
\hline Sb-125 & & & & & & & & & & & & & & & \\
\hline Sn-126 & 92E-07 & $8.92 \mathrm{E}-07$ & $8.92 \mathrm{E}-07$ & $8.92 \mathrm{E}-07$ & $8.92 \mathrm{E}-07$ & $8.92 \mathrm{E}-07$ & $8.92 \mathrm{E}-07$ & $8.92 \mathrm{E}-07$ & $8.92 \mathrm{E}-07$ & $8.92 \mathrm{E}-07$ & $8.92 \mathrm{E}-07$ & $8.92 \mathrm{E}-07$ & $8.92 \mathrm{E}-07$ & $8.92 \mathrm{E}-07$ & 8.9 \\
\hline I-129 & $1.04 \mathrm{E}-10$ & $1.04 \mathrm{E}-10$ & $1.04 \mathrm{E}-10$ & $1.04 \mathrm{E}-10$ & $1.04 \mathrm{E}-10$ & $1.04 \mathrm{E}-10$ & $1.04 \mathrm{E}-10$ & $1.04 \mathrm{E}-10$ & $1.04 \mathrm{E}-10$ & $1.04 \mathrm{E}-10$ & $1.04 \mathrm{E}-10$ & $1.04 \mathrm{E}-10$ & $1.04 \mathrm{E}-10$ & $1.04 \mathrm{E}-10$ & \\
\hline Cs-134 & $4.70 \mathrm{E}-06$ & $4.70 \mathrm{E}-06$ & $4.70 \mathrm{E}-06$ & $4.70 \mathrm{E}-06$ & $4.70 \mathrm{E}-06$ & & $4.70 \mathrm{E}-06$ & $4.70 \mathrm{E}-06$ & $4.70 \mathrm{E}-06$ & $4.70 \mathrm{E}-06$ & $0 \mathrm{E}-06$ & $4.70 \mathrm{E}-06$ & $\mathrm{E}-06$ & $0 \mathrm{E}-06$ & $4.70 \mathrm{E}-0$ \\
\hline Cs-135 & & 7.98 & & & & & & & & & & & & & \\
\hline Cs-137 & $39 \mathrm{E}-02$ & $3.23 \mathrm{E}-02$ & $3.52 \mathrm{E}-02$ & $3.04 \mathrm{E}-02$ & $7.94 \mathrm{E}-02$ & & & $44 \mathrm{E}-01$ & $E-01$ & $4.23 \mathrm{E}-03$ & & $4.12 \mathrm{E}-02$ & $3.91 \mathrm{E}-02$ & $7 \mathrm{E}-02$ & \\
\hline Ba-137m & $3.21 \mathrm{E}-02$ & $3.05 \mathrm{E}-02$ & $3.33 \mathrm{E}-02$ & $2.87 \mathrm{E}-02$ & $7.51 \mathrm{E}-02$ & & & $1.36 \mathrm{E}-01$ & $\mathrm{E}-02$ & $4.00 \mathrm{E}-03$ & $\mathrm{E}-02$ & & & $1 \mathrm{E}-02$ & $2.09 \mathrm{E}-0$ \\
\hline Sr-90 & $3.93 \mathrm{E}-02$ & $3.93 \mathrm{E}-02$ & $3.93 \mathrm{E}-02$ & $3.94 \mathrm{E}-02$ & $3.93 \mathrm{E}-02$ & & $\mathrm{E}-02$ & $3.93 \mathrm{E}-02$ & E-02 & $3.93 \mathrm{E}-02$ & $3.93 \mathrm{E}-02$ & $3.93 \mathrm{E}-02$ & $3.93 \mathrm{E}-02$ & $3.93 \mathrm{E}-02$ & $3.93 \mathrm{E}-0$ \\
\hline$Y-90$ & $3.93 \mathrm{E}-02$ & $3.93 \mathrm{E}-02$ & $3.93 \mathrm{E}-02$ & $3.94 \mathrm{E}-02$ & $3.93 \mathrm{E}-02$ & $3.94 \mathrm{E}-02$ & $3.94 \mathrm{E}-02$ & $3.93 \mathrm{E}-02$ & $3.93 \mathrm{E}-02$ & $3.93 \mathrm{E}-02$ & $3.93 \mathrm{E}-02$ & $3.93 \mathrm{E}-02$ & $3.93 \mathrm{E}-02$ & $3.93 \mathrm{E}-02$ & $3.93 \mathrm{E}-0$ \\
\hline $\mathrm{H}-3$ & & -- & 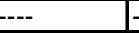 & & & & & & & & & & & & \\
\hline Ce-144 & $2.16 \mathrm{E}-06$ & $2.16 \mathrm{E}-06$ & $2.16 \mathrm{E}-06$ & $2.16 \mathrm{E}-06$ & $2.16 \mathrm{E}-06$ & & & $2.16 \mathrm{E}-06$ & $2.16 \mathrm{E}-06$ & & & & & $2.16 \mathrm{E}-06$ & \\
\hline Pr-144 & $2.16 \mathrm{E}-06$ & $2.16 \mathrm{E}-06$ & $2.16 \mathrm{E}-06$ & $2.16 \mathrm{E}-06$ & $2.16 \mathrm{E}-06$ & & $=-06$ & -06 & E-06 & & -06 & & & $\mathrm{E}-06$ & $6 \mathrm{E}-0$ \\
\hline Pm-147 & & & & & & & & & & & & & & & \\
\hline Eu-154 & -04 & $E-04$ & $E-04$ & $E-04$ & $4.41 \mathrm{E}-04$ & & & E-04 & $\mathrm{E}-04$ & & $E-04$ & & & $E-04$ & 4.41 \\
\hline Th-232 & & 1.27 & & & 1.27 & & & & & & & & & & \\
\hline $\mathrm{U}-232$ & 2.99 & 2.99 & & 2.74 & & & & & & & & & & & \\
\hline $\mathrm{U}-233$ & & 4.29 & & & $=-08$ & & & & & & & & & & \\
\hline $\mathrm{U}-234$ & & & & & & & & & & & & & & & \\
\hline $\mathrm{U}-235$ & & 7.34 & & & & & & & & & & & & & $7.35 \mathrm{E}-$ \\
\hline $\mathrm{U}-236$ & & & & & & & & & & & & & & & \\
\hline $\mathrm{U}-238$ & & $2.60 \mathrm{E}-08$ & & $2.08 \mathrm{E}-08$ & $2.08 \mathrm{E}-08$ & & & & & & -08 & & & & \\
\hline Np-237 & & & & & & & & & & & & & & & \\
\hline Pu-238 & & & & & & & & & & & & & & & \\
\hline Pu-239 & & & & & & & & & & & & & & & \\
\hline Pu-240 & & $8.05 \mathrm{E}-06$ & & 6.68 & 6.68 & & & & & & & & & $=-06$ & \\
\hline Pu-241 & & & & & & & & & & & & & & & \\
\hline Pu-242 & & & & & & & & & & & & & & & \\
\hline Am-241 & & $9.42 \mathrm{E}-05$ & $9.42 \mathrm{E}-05$ & & $E-05$ & & & $E-05$ & $\mathrm{E}-05$ & & & & & $\mathrm{E}-05$ & $9.42 \mathrm{E}-$ \\
\hline Am-24 & $8.85 \mathrm{E}-08$ & $8.85 \mathrm{E}-08$ & $8.85 \mathrm{E}-08$ & $8.89 \mathrm{E}-08$ & $8.89 \mathrm{E}-08$ & $1.52 \mathrm{E}-07$ & $1.52 \mathrm{E}-07$ & $8.89 \mathrm{E}-08$ & $8.89 \mathrm{E}-08$ & $8.89 \mathrm{E}-08$ & $8.89 \mathrm{E}-08$ & $8.85 \mathrm{E}-08$ & $8.85 \mathrm{E}-08$ & $8.85 \mathrm{E}-08$ & $8.85 \mathrm{E}-0$ \\
\hline & & & & & & & & & & & & & & & \\
\hline & & & & & & & & & & & & & & & \\
\hline $\mathrm{Cm}-245$ & & & & & & & & & & & & & & E-09 & \\
\hline Total Alpha & $1.10 \mathrm{E}-03$ & $1.10 \mathrm{E}-03$ & $1.10 \mathrm{E}-03$ & $1.05 \mathrm{E}-03$ & $1.05 \mathrm{E}-03$ & $1.10 \mathrm{E}-03$ & $1.10 \mathrm{E}-03$ & $1.05 \mathrm{E}-03$ & $1.05 \mathrm{E}-03$ & $1.10 \mathrm{E}-03$ & 1.09E-03 & $1.10 \mathrm{E}-03$ & $1.10 \mathrm{E}-03$ & $1.10 \mathrm{E}-03$ & $1.10 \mathrm{E}-03$ \\
\hline
\end{tabular}


Table 14: Projected Feed Composition After Dissolution and Dilution to 6.44 M Total Sodium Concentration $100 \mathrm{mg} / \mathrm{L}$ sludge; $10 \%$ of interstitial supernate remaining

\begin{tabular}{|c|c|c|c|c|c|c|c|c|c|c|c|c|c|c|c|}
\hline \multicolumn{16}{|c|}{ Concentration (M) } \\
\hline & Tank 25 & Tank 27 & Tank 28 & Tank 29 & Tank 31 & Tank 33 & Tank 34 & Tank 36 & Tank 37 & Tank 38 & Tank 41 & Tank 44 & Tank 45 & Tank 46 & Tank 47 \\
\hline $\mathrm{Na}$ & $6.44 \mathrm{E}+00$ & $6.44 \mathrm{E}+00$ & $6.44 \mathrm{E}+00$ & $6.44 \mathrm{E}+00$ & $6.44 \mathrm{E}+00$ & $6.44 \mathrm{E}+00$ & $6.44 \mathrm{E}+00$ & $6.44 \mathrm{E}+00$ & $6.44 \mathrm{E}+00$ & $6.44 \mathrm{E}+00$ & $6.44 \mathrm{E}+00$ & $6.44 \mathrm{E}+00$ & $6.44 \mathrm{E}+00$ & $6.44 \mathrm{E}+00$ & $6.44 \mathrm{E}+00$ \\
\hline $\mathrm{Al}(\mathrm{OH}) 4$ & $3.22 \mathrm{E}-01$ & $3.25 \mathrm{E}-01$ & $3.22 \mathrm{E}-01$ & 3.99E-01 & $4.00 \mathrm{E}-01$ & $3.21 \mathrm{E}-01$ & $3.20 \mathrm{E}-01$ & $3.94 \mathrm{E}-01$ & 3.97E-01 & $4.00 \mathrm{E}-01$ & 3.96E-01 & 3.20E-01 & $3.19 \mathrm{E}-01$ & $3.17 \mathrm{E}-01$ & $3.22 \mathrm{E}-01$ \\
\hline $\mathrm{CO} 3$ & $2.22 \mathrm{E}-01$ & $2.22 \mathrm{E}-01$ & $2.21 \mathrm{E}-01$ & $1.33 \mathrm{E}-01$ & $1.32 \mathrm{E}-01$ & $2.23 \mathrm{E}-01$ & $2.25 \mathrm{E}-01$ & $1.33 \mathrm{E}-01$ & $1.32 \mathrm{E}-01$ & $1.34 \mathrm{E}-01$ & $1.34 \mathrm{E}-01$ & $2.22 \mathrm{E}-01$ & $2.23 \mathrm{E}-01$ & $2.20 \mathrm{E}-01$ & $2.24 \mathrm{E}-01$ \\
\hline $\mathrm{C2O4}$ & $2.23 \mathrm{E}-02$ & $2.23 \mathrm{E}-02$ & $2.23 \mathrm{E}-02$ & $1.42 \mathrm{E}-02$ & $1.41 \mathrm{E}-02$ & $2.25 \mathrm{E}-02$ & $2.23 \mathrm{E}-02$ & $1.41 \mathrm{E}-02$ & $1.41 \mathrm{E}-02$ & $1.42 \mathrm{E}-02$ & $1.41 \mathrm{E}-02$ & $2.22 \mathrm{E}-02$ & $2.23 \mathrm{E}-02$ & $2.22 \mathrm{E}-02$ & $2.24 \mathrm{E}-02$ \\
\hline NO2 & $1.11 \mathrm{E}-01$ & $1.11 \mathrm{E}-01$ & $1.14 \mathrm{E}-01$ & $7.44 \mathrm{E}-01$ & $7.49 \mathrm{E}-01$ & $1.02 \mathrm{E}-01$ & $1.10 \mathrm{E}-01$ & $7.47 \mathrm{E}-01$ & $7.45 \mathrm{E}-01$ & $7.43 \mathrm{E}-01$ & $7.41 \mathrm{E}-01$ & $1.12 \mathrm{E}-01$ & $1.13 \mathrm{E}-01$ & $1.12 \mathrm{E}-01$ & $1.06 \mathrm{E}-01$ \\
\hline NO3 & $3.80 \mathrm{E}+00$ & $3.80 \mathrm{E}+00$ & $3.80 \mathrm{E}+00$ & $2.66 \mathrm{E}+00$ & $2.65 \mathrm{E}+00$ & $3.84 \mathrm{E}+00$ & $3.81 \mathrm{E}+00$ & $2.65 \mathrm{E}+00$ & $2.65 \mathrm{E}+00$ & $2.67 \mathrm{E}+00$ & $2.67 \mathrm{E}+00$ & $3.79 \mathrm{E}+00$ & $3.79 \mathrm{E}+00$ & $3.79 \mathrm{E}+00$ & $3.82 \mathrm{E}+00$ \\
\hline $\mathrm{OH}$ & $9.63 \mathrm{E}-01$ & $9.65 \mathrm{E}-01$ & $9.47 \mathrm{E}-01$ & $1.85 \mathrm{E}+00$ & $1.86 \mathrm{E}+00$ & $9.14 \mathrm{E}-01$ & $9.42 \mathrm{E}-01$ & $1.89 \mathrm{E}+00$ & $1.87 \mathrm{E}+00$ & $1.85 \mathrm{E}+00$ & $1.85 \mathrm{E}+00$ & $9.80 \mathrm{E}-01$ & $9.90 \mathrm{E}-01$ & $9.39 \mathrm{E}-01$ & $9.27 \mathrm{E}-01$ \\
\hline PO4 & $1.42 \mathrm{E}-03$ & $1.42 \mathrm{E}-03$ & $1.46 \mathrm{E}-03$ & $1.57 \mathrm{E}-02$ & 1.57E-02 & $1.34 \mathrm{E}-03$ & $1.34 \mathrm{E}-03$ & $1.57 \mathrm{E}-02$ & $1.56 \mathrm{E}-02$ & $1.57 \mathrm{E}-02$ & $1.57 \mathrm{E}-02$ & $1.37 \mathrm{E}-03$ & $1.38 \mathrm{E}-03$ & $1.41 \mathrm{E}-03$ & $1.34 \mathrm{E}-03$ \\
\hline SO4 & $3.25 \mathrm{E}-01$ & $3.25 \mathrm{E}-01$ & $3.25 \mathrm{E}-01$ & $1.84 \mathrm{E}-01$ & $1.83 \mathrm{E}-01$ & $3.29 \mathrm{E}-01$ & $3.26 \mathrm{E}-01$ & $1.83 \mathrm{E}-01$ & $1.83 \mathrm{E}-01$ & $1.85 \mathrm{E}-01$ & $1.83 \mathrm{E}-01$ & $3.24 \mathrm{E}-01$ & $3.25 \mathrm{E}-01$ & 3.24E-01 & 3.27E-01 \\
\hline $\mathbf{A g}$ & $6.13 \mathrm{E}-09$ & $3.60 \mathrm{E}-08$ & $2.65 \mathrm{E}-08$ & $1.33 \mathrm{E}-08$ & $1.14 \mathrm{E}-08$ & $1.25 \mathrm{E}-08$ & $1.24 \mathrm{E}-08$ & $1.14 \mathrm{E}-08$ & $1.14 \mathrm{E}-08$ & $1.46 \mathrm{E}-08$ & $1.15 \mathrm{E}-08$ & $1.24 \mathrm{E}-08$ & $1.24 \mathrm{E}-08$ & $1.23 \mathrm{E}-08$ & $1.24 \mathrm{E}-08$ \\
\hline As & $1.08 \mathrm{E}-06$ & 9.79E-07 & $1.27 \mathrm{E}-06$ & $1.73 \mathrm{E}-06$ & $1.80 \mathrm{E}-05$ & $8.63 \mathrm{E}-06$ & $2.21 \mathrm{E}-05$ & $3.13 \mathrm{E}-05$ & $2.25 \mathrm{E}-05$ & $1.19 \mathrm{E}-06$ & $1.48 \mathrm{E}-05$ & $3.75 \mathrm{E}-05$ & $4.11 \mathrm{E}-05$ & $2.26 \mathrm{E}-05$ & $1.56 \mathrm{E}-05$ \\
\hline $\mathrm{Ba}$ & 1.07E-09 & $1.07 \mathrm{E}-08$ & $8.02 \mathrm{E}-09$ & $6.97 \mathrm{E}-08$ & ---- & --- & ---- & --- & ---- & $2.50 \mathrm{E}-09$ & --- & --- & --- & --- & --- \\
\hline $\mathbf{C a}$ & 5.05E-05 & $5.04 \mathrm{E}-05$ & $5.05 \mathrm{E}-05$ & $3.15 \mathrm{E}-05$ & 3.09E-05 & 5.15E-05 & $5.06 \mathrm{E}-05$ & 3.09E-05 & $3.09 \mathrm{E}-05$ & $3.17 \mathrm{E}-05$ & $3.09 \mathrm{E}-01$ & $5.04 \mathrm{E}-05$ & 5.04E-05 & 5.03E-05 & $5.08 \mathrm{E}-05$ \\
\hline Cd & $2.94 \mathrm{E}-08$ & $2.81 \mathrm{E}-08$ & $3.72 \mathrm{E}-08$ & $4.56 \mathrm{E}-08$ & $4.85 \mathrm{E}-07$ & $1.03 \mathrm{E}-07$ & $6.26 \mathrm{E}-07$ & $9.98 \mathrm{E}-07$ & $6.59 \mathrm{E}-07$ & $2.44 \mathrm{E}-08$ & $3.62 \mathrm{E}-07$ & $1.22 \mathrm{E}-06$ & $1.36 \mathrm{E}-06$ & $6.47 \mathrm{E}-07$ & $3.72 \mathrm{E}-07$ \\
\hline $\mathrm{Cl}$ & $4.27 \mathrm{E}-02$ & $4.27 \mathrm{E}-02$ & $4.27 \mathrm{E}-02$ & $3.86 \mathrm{E}-02$ & $3.83 \mathrm{E}-02$ & $4.30 \mathrm{E}-02$ & & $\mathrm{E}-02$ & E-02 & $3.87 \mathrm{E}-02$ & E-02 & $4.26 \mathrm{E}-02$ & $4.27 \mathrm{E}-02$ & $4.26 \mathrm{E}-02$ & $4.28 \mathrm{E}-02$ \\
\hline Co & $1.57 \mathrm{E}-07$ & $1.60 \mathrm{E}-07$ & $1.32 \mathrm{E}-07$ & -08 & $1.03 \mathrm{E}-07$ & $6.64 \mathrm{E}-08$ & & E-07 & E-07 & $6.66 \mathrm{E}-08$ & $\mathrm{E}-08$ & $1.86 \mathrm{E}-07$ & $0 \mathrm{E}-07$ & $1.24 \mathrm{E}-07$ & $9.49 \mathrm{E}-08$ \\
\hline $\mathrm{Cr}$ & $6.50 \mathrm{E}-06$ & $3.95 \mathrm{E}-06$ & $1.31 \mathrm{E}-05$ & $5.35 \mathrm{E}-05$ & $7.19 \mathrm{E}-05$ & $8.77 \mathrm{E}-05$ & $7.59 \mathrm{E}-05$ & $6.12 \mathrm{E}-05$ & $6.83 \mathrm{E}-05$ & $6.72 \mathrm{E}-05$ & $7.48 \mathrm{E}-05$ & $6.31 \mathrm{E}-05$ & $6.03 \mathrm{E}-05$ & $7.51 \mathrm{E}-05$ & $8.15 \mathrm{E}-05$ \\
\hline $\mathrm{Cu}$ & $5.62 \mathrm{E}-05$ & $5.82 \mathrm{E}-05$ & $4.25 \mathrm{E}-05$ & $1.01 \mathrm{E}-05$ & $2.88 \mathrm{E}-05$ & $6.17 \mathrm{E}-06$ & $3.71 \mathrm{E}-05$ & $5.91 \mathrm{E}-05$ & $3.91 \mathrm{E}-05$ & $8.57 \mathrm{E}-06$ & $2.15 \mathrm{E}-05$ & $7.23 \mathrm{E}-05$ & $8.05 \mathrm{E}-05$ & $3.84 \mathrm{E}-05$ & $2.21 \mathrm{E}-05$ \\
\hline $\mathrm{Fe}$ & $4.73 \mathrm{E}-07$ & $4.73 \mathrm{E}-07$ & $3.68 \mathrm{E}-07$ & E-07 & $1.31 \mathrm{E}-06$ & E-07 & & E-06 & $\mathrm{E}-06$ & $6.51 \mathrm{E}-07$ & -07 & $3.24 \mathrm{E}-06$ & E-06 & $1.74 \mathrm{E}-06$ & $1.02 \mathrm{E}-06$ \\
\hline $\mathbf{F}$ & $7.12 \mathrm{E}-02$ & $7.11 \mathrm{E}-02$ & $7.12 \mathrm{E}-02$ & $3.62 \mathrm{E}-02$ & $3.60 \mathrm{E}-02$ & $7.19 \mathrm{E}-02$ & $\mathrm{E}-02$ & $3.60 \mathrm{E}-02$ & $3.60 \mathrm{E}-02$ & $3.63 \mathrm{E}-02$ & $3.60 \mathrm{E}-02$ & $7.11 \mathrm{E}-02$ & $1 \mathrm{E}-02$ & $7.09 \mathrm{E}-02$ & $7.15 \mathrm{E}-02$ \\
\hline $\mathrm{Hg}$ & $3.66 \mathrm{E}-07$ & $3.66 \mathrm{E}-07$ & $3.66 \mathrm{E}-07$ & $6.82 \mathrm{E}-07$ & $4.16 \mathrm{E}-06$ & $4.32 \mathrm{E}-06$ & $4.56 \mathrm{E}-06$ & $4.43 \mathrm{E}-06$ & $4.25 \mathrm{E}-06$ & $3.42 \mathrm{E}-07$ & $4.10 \mathrm{E}-06$ & $4.86 \mathrm{E}-06$ & $4.94 \mathrm{E}-06$ & $4.55 \mathrm{E}-06$ & 4.44E-06 \\
\hline $\mathrm{K}$ & $5.74 \mathrm{E}-03$ & $5.81 \mathrm{E}-03$ & $5.74 \mathrm{E}-03$ & $4.74 \mathrm{E}-03$ & $4.94 \mathrm{E}-03$ & $4.93 \mathrm{E}-03$ & $5.35 \mathrm{E}-03$ & $5.57 \mathrm{E}-03$ & $5.01 \mathrm{E}-03$ & $4.56 \mathrm{E}-03$ & $4.68 \mathrm{E}-03$ & $5.95 \mathrm{E}-03$ & $6.10 \mathrm{E}-03$ & $5.40 \mathrm{E}-03$ & $5.20 \mathrm{E}-03$ \\
\hline $\mathrm{Mg}$ & --- & --- & $3.45 \mathrm{E}-09$ & $4.40 \mathrm{E}-08$ & $1.77 \mathrm{E}-08$ & $5.43 \mathrm{E}-08$ & $\mathrm{E}-08$ & --- & E-09 & $4.63 \mathrm{E}-08$ & E-08 & --- & ---- & $9.03 \mathrm{E}-09$ & $3.19 \mathrm{E}-08$ \\
\hline $\mathrm{Mn}$ & $2.01 \mathrm{E}-08$ & $2.40 \mathrm{E}-08$ & $3.21 \mathrm{E}-08$ & $3.73 \mathrm{E}-08$ & $6.77 \mathrm{E}-08$ & $3.51 \mathrm{E}-08$ & & $1.15 \mathrm{E}-07$ & $\mathrm{E}-08$ & $2.00 \mathrm{E}-08$ & E-08 & $1.37 \mathrm{E}-07$ & $1.49 \mathrm{E}-07$ & $8.44 \mathrm{E}-08$ & $5.95 \mathrm{E}-08$ \\
\hline Nd & --- & ---- & ---- & $1.46 \mathrm{E}-06$ & ---- & $1.96 \mathrm{E}-06$ & ---- & --- & ---- & $1.59 \mathrm{E}-06$ & $5.41 \mathrm{E}-07$ & ---- & ---- & $\begin{array}{l}--- \\
\end{array}$ & $6.81 \mathrm{E}-07$ \\
\hline $\mathbf{N i}$ & $3.75 \mathrm{E}-07$ & 3.84E-07 & \begin{tabular}{|c|}
$3.09 \mathrm{E}-07$ \\
\end{tabular} & $1.45 \mathrm{E}-07$ & 2.34E-07 & $1.34 \mathrm{E}-07$ & $2.83 \mathrm{E}-07$ & $3.81 \mathrm{E}-07$ & $2.84 \mathrm{E}-07$ & $1.38 \mathrm{E}-07$ & $2.00 \mathrm{E}-07$ & $4.52 \mathrm{E}-07$ & $4.92 \mathrm{E}-07$ & $2.88 \mathrm{E}-07$ & $2.11 \mathrm{E}-07$ \\
\hline $\mathrm{Pb}$ & $1.03 \mathrm{E}-06$ & $8.50 \mathrm{E}-07$ & 1.17E-06 & $1.68 \mathrm{E}-06$ & ---- & ---- & ---- & ---- & ---- & 9.93E-07 & --- & ---- & ---- & \begin{tabular}{|l|}
---- \\
\end{tabular} & ---- \\
\hline $\mathbf{R u}$ & $5.94 \mathrm{E}-06$ & $6.12 \mathrm{E}-06$ & $4.60 \mathrm{E}-06$ & $1.40 \mathrm{E}-06$ & \begin{tabular}{|l|}
$3.22 \mathrm{E}-06$ \\
\end{tabular} & $1.06 \mathrm{E}-06$ & $\mathrm{E}-06$ & $\mathrm{E}-06$ & $4.23 \mathrm{E}-06$ & $1.26 \mathrm{E}-06$ & E-06 & $7.51 \mathrm{E}-06$ & $8.30 \mathrm{E}-06$ & $4.19 \mathrm{E}-06$ & $2.61 \mathrm{E}-06$ \\
\hline $\mathrm{Se}$ & $7.63 \mathrm{E}-06$ & $5.85 \mathrm{E}-06$ & $9.85 \mathrm{E}-06$ & $2.03 \mathrm{E}-05$ & $5.53 \mathrm{E}-05$ & $7.32 \mathrm{E}-05$ & E-05 & $0 \mathrm{E}-05$ & $1 \mathrm{E}-05$ & $1.96 \mathrm{E}-05$ & $5.91 \mathrm{E}-05$ & $3.90 \mathrm{E}-05$ & $0 \mathrm{E}-05$ & $5.60 \mathrm{E}-05$ & $6.47 \mathrm{E}-05$ \\
\hline $\mathbf{S i}$ & $3.64 \mathrm{E}-05$ & $3.19 \mathrm{E}-05$ & $1.86 \mathrm{E}-04$ & $6.09 \mathrm{E}-06$ & ---- & $\cdots-$ & ---- & $\cdots$ & --- & $4.76 \mathrm{E}-05$ & --- & 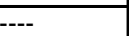 & $\cdots$ & --- & -- \\
\hline $\mathrm{Sr}$ & --- & -- & 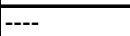 & $9.83 \mathrm{E}-07$ & $-\cdots-$ & $1.36 \mathrm{E}-06$ & $-\cdots-$ & $-\cdots$ & $-\cdots-$ & $1.08 \mathrm{E}-06$ & $2.71 \mathrm{E}-07$ & --- & $\ldots-$ & --- & 3.67E-07 \\
\hline $\mathrm{Zn}$ & $6.83 \mathrm{E}-05$ & $7.04 \mathrm{E}-05$ & $5.34 \mathrm{E}-05$ & $1.76 \mathrm{E}-05$ & 3.79E-05 & $1.40 \mathrm{E}-05$ & $4.75 \mathrm{E}-05$ & $7.09 \mathrm{E}-05$ & $4.91 \mathrm{E}-05$ & $1.60 \mathrm{E}-05$ & $3.00 \mathrm{E}-05$ & $8.58 \mathrm{E}-05$ & $9.46 \mathrm{E}-05$ & \begin{tabular}{|l}
$4.89 \mathrm{E}-05$ \\
\end{tabular} & $3.12 \mathrm{E}-05$ \\
\hline$\overline{Z r}$ & $1.80 \mathrm{E}-06$ & $1.86 \mathrm{E}-06$ & $1.37 \mathrm{E}-06$ & $3.56 \mathrm{E}-07$ & $9.39 \mathrm{E}-07$ & $2.39 \mathrm{E}-07$ & $1.20 \mathrm{E}-06$ & $1.88 \mathrm{E}-06$ & $1.26 \mathrm{E}-06$ & $3.10 \mathrm{E}-07$ & $7.12 \mathrm{E}-07$ & $2.30 \mathrm{E}-06$ & $2.55 \mathrm{E}-06$ & $1.24 \mathrm{E}-06$ & $7.35 \mathrm{E}-07$ \\
\hline Insol.Sol.m & 100 & 100 & 100 & 100 & 100 & 100 & 100 & 100 & 100 & 100 & 100 & 100 & 100 & 100 & 100 \\
\hline
\end{tabular}


Table 14: Projected Feed Composition After Dissolution and Dilution to 6.44 M Total Sodium Concentration $100 \mathrm{mg} / \mathrm{L}$ sludge; $10 \%$ of interstitial supernate remaining (continued)

\begin{tabular}{|c|c|c|c|c|c|c|c|c|c|c|c|c|c|c|c|}
\hline \\
\hline & Tank 25 & Tank 27 & Tank 28 & Tank 29 & Tank 31 & Tank 33 & Tank 34 & Tank 36 & Tank 37 & & Ialin 41 & Tank 44 & Tank 45 & Tank 46 & Tank 47 \\
\hline C-14 & $5.62 \mathrm{E}-10$ & $5.62 \mathrm{E}-10$ & $5.62 \mathrm{E}-10$ & $5.62 \mathrm{E}-10$ & $5.62 \mathrm{E}-10$ & $5.62 \mathrm{E}-10$ & $5.62 \mathrm{E}-10$ & $5.62 \mathrm{E}-10$ & $5.62 \mathrm{E}-10$ & $5.62 \mathrm{E}-10$ & $5.62 \mathrm{E}-10$ & $5.62 \mathrm{E}-10$ & $5.62 \mathrm{E}-10$ & $5.62 \mathrm{E}-10$ & $5.62 \mathrm{E}-1 \mathrm{C}$ \\
\hline $\mathrm{Ni}-59$ & $3.23 \mathrm{E}-07$ & $3.23 \mathrm{E}-07$ & $3.23 \mathrm{E}-07$ & $3.23 \mathrm{E}-07$ & $3.23 \mathrm{E}-07$ & $3.23 \mathrm{E}-07$ & $3.23 \mathrm{E}-07$ & $3.23 \mathrm{E}-07$ & $3.23 \mathrm{E}-07$ & $3.23 \mathrm{E}-07$ & $3.23 \mathrm{E}-07$ & 3.23E-07 & $3.23 \mathrm{E}-07$ & 3.23E-07 & \\
\hline $\mathrm{Ni}-63$ & $5.02 \mathrm{E}-10$ & $5.02 \mathrm{E}-10$ & $5.02 \mathrm{E}-10$ & $5.02 \mathrm{E}-10$ & $5.02 \mathrm{E}-10$ & $5.02 \mathrm{E}-10$ & $5.02 \mathrm{E}-10$ & $5.02 \mathrm{E}-10$ & $5.02 \mathrm{E}-10$ & $5.02 \mathrm{E}-10$ & $5.02 \mathrm{E}-10$ & $5.02 \mathrm{E}-10$ & $5.02 \mathrm{E}-10$ & & $5.02 \mathrm{E}$ \\
\hline Co-60 & $5.14 \mathrm{E}-05$ & $5.14 \mathrm{E}-05$ & $5.14 \mathrm{E}-05$ & $5.14 \mathrm{E}-05$ & $5.14 \mathrm{E}-05$ & $5.14 \mathrm{E}-05$ & $5.14 \mathrm{E}-05$ & 14E-05 & $5.14 \mathrm{E}-05$ & $5.14 \mathrm{E}-05$ & & 5 514F-0 & & $5.14 \mathrm{E}-0$ & $.14 \mathrm{E}$ \\
\hline Se-79 & $1.91 \mathrm{E}-07$ & $1.91 \mathrm{E}-07$ & $1.91 \mathrm{E}-07$ & $1.91 \mathrm{E}-07$ & $1.91 \mathrm{E}-07$ & $1.91 \mathrm{E}-07$ & $1.91 \mathrm{E}-07$ & & & & & & & & $1.91 \mathrm{E}$ \\
\hline Tc-99 & $3.24 \mathrm{E}-06$ & $3.24 \mathrm{E}-06$ & $3.24 \mathrm{E}-06$ & $3.24 \mathrm{E}-06$ & $3.24 \mathrm{E}-06$ & $3.24 \mathrm{E}-06$ & $3.24 \mathrm{E}-06$ & $3.24 \mathrm{E}-06$ & $3.24 \mathrm{E}-06$ & $3.24 \mathrm{E}-06$ & $3.24 \mathrm{E}-06$ & $3.24 \mathrm{E}-06$ & $3.24 \mathrm{E}-06$ & $3.24 \mathrm{E}-06$ & $3.24 \mathrm{E}-06$ \\
\hline Ru-106 & $1.20 \mathrm{E}-06$ & $1.20 \mathrm{E}-06$ & $1.20 \mathrm{E}-06$ & $1.20 \mathrm{E}-06$ & $1.20 \mathrm{E}-06$ & $1.20 \mathrm{E}-06$ & $1.20 \mathrm{E}-06$ & $1.20 \mathrm{E}-06$ & $1.20 \mathrm{E}-06$ & $1.20 \mathrm{E}-06$ & $1.20 \mathrm{E}-00$ & $1.20 \mathrm{E}-06$ & $1.20 \mathrm{E}-06$ & $1.20 \mathrm{E}-06$ & $1.20 \mathrm{E}-$ \\
\hline Rh-106 & $1.20 \mathrm{E}-06$ & $1.20 \mathrm{E}-06$ & $1.20 \mathrm{E}-06$ & $1.20 \mathrm{E}-06$ & $1.20 \mathrm{E}-06$ & & $1.20 \mathrm{E}-06$ & $1.20 \mathrm{E}-06$ & & & $1.20 \mathrm{E}-06$ & & & & $1.20 \mathrm{E}-0$ \\
\hline Sb-125 & $3.07 \mathrm{E}-05$ & $3.07 \mathrm{E}-05$ & $3.07 \mathrm{E}-05$ & $3.07 \mathrm{E}-05$ & 3.07E-05 & & & & $3.07 \mathrm{E}-05$ & & & & & & \\
\hline Sn-126 & $2.55 \mathrm{E}-07$ & $2.55 \mathrm{E}-07$ & $2.55 \mathrm{E}-07$ & $2.55 \mathrm{E}-07$ & $2.55 \mathrm{E}-07$ & $2.55 \mathrm{E}-07$ & $2.55 \mathrm{E}-07$ & $2.55 \mathrm{E}-07$ & $2.55 \mathrm{E}-07$ & $2.55 \mathrm{E}-07$ & $2.55 \mathrm{E}-07$ & $2.55 \mathrm{E}-07$ & $2.55 \mathrm{E}-07$ & $2.55 \mathrm{E}-07$ & $2.55 \mathrm{E}-07$ \\
\hline |-129 & $2.98 \mathrm{E}-11$ & $2.98 \mathrm{E}-11$ & $2.98 \mathrm{E}-11$ & $2.98 \mathrm{E}-11$ & $2.98 \mathrm{E}-11$ & $2.98 \mathrm{E}-11$ & $2.98 \mathrm{E}-11$ & $2.98 \mathrm{E}-11$ & $2.98 \mathrm{E}-11$ & $2.98 \mathrm{E}-11$ & $2.98 \mathrm{E}-11$ & $2.98 \mathrm{E}-11$ & $2.98 \mathrm{E}-11$ & $2.98 \mathrm{E}-11$ & $2.98 \mathrm{E}-11$ \\
\hline Cs-134 & $1.34 \mathrm{E}-06$ & $1.34 \mathrm{E}-06$ & $1.34 \mathrm{E}-06$ & $1.34 \mathrm{E}-06$ & $1.34 \mathrm{E}-06$ & & & & & & & & & & \\
\hline Cs-135 & $2.2 \varepsilon$ & & & 2.28 & $2.28 \mathrm{E}-0 \mathrm{~s}$ & & & & & & & & & & \\
\hline Cs-137 & $3.22 \mathrm{E}-02$ & $3.06 \mathrm{E}-02$ & $3.35 \mathrm{E}-02$ & $2.87 \mathrm{E}-02$ & $7.77 \mathrm{E}-02$ & & 2.63 & $1.42 \mathrm{E}-01$ & $1.04 \mathrm{E}-01$ & $2.53 \mathrm{E}-03$ & $1.78 \mathrm{E}-02$ & $3.95 \mathrm{E}-02$ & $4 \mathrm{E}-02$ & $4.80 \mathrm{E}-02$ & $2.04 \mathrm{E}$ \\
\hline Ba-137m & $3.05 \mathrm{E}-02$ & $2.89 \mathrm{E}-02$ & $3.17 \mathrm{E}-02$ & $2.71 \mathrm{E}-02$ & $7.35 \mathrm{E}-02$ & & 2.49 & $1.35 \mathrm{E}-01$ & $9.80 \mathrm{E}-02$ & $2.40 \mathrm{E}-03$ & $1.69 \mathrm{E}-02$ & $3.74 \mathrm{E}-02$ & $3.54 \mathrm{E}-02$ & $4.55 \mathrm{E}-02$ & $1.93 \mathrm{E}-$ \\
\hline Sr-90 & $\frac{1.0 \mathrm{~L}-02}{1.12 \mathrm{E}-02}$ & $\frac{1.12 \mathrm{E}-02}{1.0}$ & $\frac{1.12 \mathrm{E}-02}{1.1}$ & $\begin{array}{l}1.13 \mathrm{E}-02 \\
\end{array}$ & $1.13 \mathrm{E}-02$ & & $\frac{2.45}{1.13}$ & $1.13 \mathrm{E}-02$ & $\begin{array}{l}1.0 \mathrm{E}-02 \\
13\end{array}$ & $\begin{array}{l}1.40 \mathrm{~L}-12 \mathrm{E} \\
\end{array}$ & $\frac{0}{1.12 \mathrm{E}-02}$ & $\frac{.14 \mathrm{~L}-02}{1.12 \mathrm{E}}$ & $\frac{0.04 L}{1.12 \mathrm{E}-02}$ & $\frac{.02}{1.12 \mathrm{E}-02}$ & $1.12 \mathrm{E}-$ \\
\hline $\begin{array}{l}\mathrm{Y}-90 \\
\text {. }\end{array}$ & $1.12 \mathrm{E}-02$ & $1.12 \mathrm{E}-02$ & $1.12 \mathrm{E}-02$ & $1.13 \mathrm{E}-02$ & $1.13 \mathrm{E}-02$ & $1.13 \mathrm{E}-02$ & $1.13 \mathrm{E}-02$ & $1.13 \mathrm{E}-02$ & $1.13 \mathrm{E}-02$ & $1.12 \mathrm{E}-02$ & $1.12 \mathrm{E}-02$ & $1.12 \mathrm{E}-02$ & $1.12 \mathrm{E}-02$ & $1.12 \mathrm{E}-02$ & $1.12 \mathrm{E}-0$ \\
\hline $\mathrm{H}-3$ & & --- & $-{ }_{-1}$ & & & & & & & & & & & & \\
\hline Ce-144 & $6.17 \mathrm{E}-07$ & $6.17 \mathrm{E}-07$ & $6.17 \mathrm{E}-07$ & $6.17 \mathrm{E}-07$ & $6.17 \mathrm{E}-07$ & & $6.17 \mathrm{E}-07$ & $6.17 \mathrm{E}-07$ & $6.17 \mathrm{E}-07$ & $6.17 \mathrm{E}-07$ & $6.17 \mathrm{E}-07$ & $6.17 \mathrm{E}-07$ & $6.17 \mathrm{E}-07$ & $6.17 \mathrm{E}-07$ & \\
\hline Pr-144 & $6.17 \mathrm{E}-07$ & $6.17 \mathrm{E}-07$ & 6.17 & 6.17 & $6.17 \mathrm{E}-07$ & & 6.17 & E-07 & $6.17 \mathrm{E}-07$ & & & & & E-07 & $P_{1}$ \\
\hline $\mathrm{Pm}-1$ & & & & & & & & & & & & & & & \\
\hline Eu-154 & 1.26 & $1.26 \mathrm{E}$ & 1.26 & 1.26 & $1.26 \mathrm{E}$ & & & -04 & $1.26 \mathrm{E}-04$ & -04 & E-04 & $1.26 \mathrm{E}-04$ & & $6 \mathrm{E}-04$ & $1.26 \mathrm{E}$ \\
\hline Th-232 & $3.62 \mathrm{E}-10$ & $3.62 \mathrm{E}-10$ & 3.62 & 3.65 & $3.65 \mathrm{E}-10$ & & & $E-10$ & $3.65 \mathrm{E}-10$ & & & 3.6 & & $3.62 \mathrm{E}-10$ & $3.62 \mathrm{E}$ \\
\hline U-232 & $1.04 \mathrm{E}-10$ & $1.04 \mathrm{E}-10$ & 1.04 & & $7.91 \mathrm{E}-11$ & & & & $7.91 \mathrm{E}-11$ & $E-11$ & & & & & \\
\hline \begin{tabular}{|l}
-233 \\
$U-233$
\end{tabular} & & $\frac{1.04 \mathrm{E}-08}{1.22 \mathrm{E}}$ & & & & & & & & & & & & & \\
\hline & & & & & & & & & & & & & & & \\
\hline $\mathrm{U}-235$ & & & & 1.96 & 1.96 & & & & & & & & & & \\
\hline $\mathrm{U}-236$ & & & & & & & & & & & & & & & \\
\hline $\mathrm{U}-238$ & 1.12 & $1.12 \mathrm{E}$ & 1.12 & $5.97 \mathrm{E}-09$ & $5.97 \mathrm{E}$ & & & & & & & & & & \\
\hline -2 & & & & & & & & & & & & & & & \\
\hline Pu-2 & -04 & & & & & & & & & & & & & E-04 & $2.82 \mathrm{E}$ \\
\hline Pu-239 & & $1.03 \mathrm{E}-05$ & & & & & & & & & & & & & \\
\hline Pu-240 & $3.29 \mathrm{E}-06$ & $3.29 \mathrm{E}-06$ & $3.29 \mathrm{E}-06$ & 1.92 & & & 2.72 & & $1.92 \mathrm{E}-06$ & & & & & & $3.29 \mathrm{E}-0$ \\
\hline-241 & & 1.67 & 1.67 & $1.30 \mathrm{E}-04$ & $1.30 \mathrm{E}-04$ & & & & & & & & & & $1.67 \mathrm{E}-0$ \\
\hline & & & & & & & & & & & & & & & \\
\hline & & & & & & & & & $2.72 \mathrm{E}-05$ & & $2.72 \mathrm{E}-05$ & $2.69 \mathrm{E}-05$ & & & \\
\hline & $2.53 \mathrm{E}-08$ & $2.53 \mathrm{E}-08$ & $2.53 \mathrm{E}-08$ & $2.57 \mathrm{E}-08$ & $2.57 \mathrm{E}-08$ & & $8.91 \mathrm{E}-08$ & $2.57 \mathrm{E}-08$ & $2.57 \mathrm{E}-08$ & $2.56 \mathrm{E}-08$ & $2.56 \mathrm{E}-08$ & $2.53 \mathrm{E}-08$ & $2.53 \mathrm{E}-08$ & $2.53 \mathrm{E}-08$ & $2.53 \mathrm{E}-0$ \\
\hline & & & & & & & & & & & & & & & \\
\hline & & & & & & & & & & & & & & & \\
\hline Cm-245 & $2.06 \mathrm{E}-09$ & $2.06 \mathrm{E}-09$ & $2.06 \mathrm{E}-09$ & $2.06 \mathrm{E}-09$ & $2.06 \mathrm{E}$ & & & & & & & & & & \\
\hline Total Alpha & $3.50 \mathrm{E}-04$ & $3.50 \mathrm{E}-04$ & $3.50 \mathrm{E}-04$ & $3.00 \mathrm{E}-04$ & $3.00 \mathrm{E}-04$ & $3.51 \mathrm{E}-04$ & $3.51 \mathrm{E}-04$ & $3.00 \mathrm{E}-04$ & $3.00 \mathrm{E}-04$ & $3.48 \mathrm{E}-04$ & $3.47 \mathrm{E}-04$ & $3.50 \mathrm{E}-04$ & $3.50 \mathrm{E}-04$ & $3.50 \mathrm{E}-04$ & $3.50 \mathrm{E}-0$ \\
\hline
\end{tabular}


Table 15: Projected Feed Composition After Dissolution and Dilution to 6.44 M Total Sodium Concentration no sludge $; 10 \%$ of interstitial supernate remaining

\begin{tabular}{|c|c|c|c|c|c|c|c|c|c|c|c|c|c|c|c|}
\hline \multicolumn{16}{|c|}{ Concentration (M) } \\
\hline & Tank 25 & Tank 27 & Tank 28 & Tank 29 & Tank 31 & Tank 33 & Tank 34 & Tank 36 & Tank 37 & Tank 38 & Tank 41 & Tank 44 & Tank 45 & Tank 46 & Tank 47 \\
\hline $\mathrm{Na}$ & $6.44 \mathrm{E}+00$ & $6.44 \mathrm{E}+00$ & $6.44 \mathrm{E}+00$ & $6.44 \mathrm{E}+00$ & $6.44 \mathrm{E}+00$ & $6.44 \mathrm{E}+00$ & $6.44 \mathrm{E}+00$ & $6.44 \mathrm{E}+00$ & $6.44 \mathrm{E}+00$ & $6.44 \mathrm{E}+00$ & $6.44 \mathrm{E}+00$ & $6.44 \mathrm{E}+00$ & $6.44 \mathrm{E}+00$ & $6.44 \mathrm{E}+00$ & $6.44 \mathrm{E}+00$ \\
\hline $\mathrm{Al}(\mathrm{OH}) 4$ & $3.22 \mathrm{E}-01$ & $3.25 \mathrm{E}-01$ & $3.22 \mathrm{E}-01$ & $3.99 \mathrm{E}-01$ & $4.00 \mathrm{E}-01$ & $3.21 \mathrm{E}-01$ & $3.20 \mathrm{E}-01$ & $3.94 \mathrm{E}-01$ & $3.97 \mathrm{E}-01$ & $4.00 \mathrm{E}-01$ & $3.96 \mathrm{E}-01$ & $3.20 \mathrm{E}-01$ & $3.19 \mathrm{E}-01$ & $3.17 \mathrm{E}-01$ & $3.22 \mathrm{E}-01$ \\
\hline $\mathrm{CO}$ & $2.22 \mathrm{E}-01$ & $2.22 \mathrm{E}-01$ & $2.21 \mathrm{E}-01$ & $1.33 \mathrm{E}-01$ & 1.32E-01 & $2.23 \mathrm{E}-01$ & & $1.33 \mathrm{E}-01$ & $1.32 \mathrm{E}-01$ & $1.34 \mathrm{E}-01$ & 1.34E-01 & $2.22 \mathrm{E}-01$ & $2.23 \mathrm{E}-01$ & $2.20 \mathrm{E}-01$ & $2.24 \mathrm{E}-01$ \\
\hline $\mathbf{C 2 O 4}$ & $2.23 \mathrm{E}-02$ & $2.23 \mathrm{E}-02$ & $2.23 \mathrm{E}-02$ & $1.42 \mathrm{E}-02$ & $1.41 \mathrm{E}-02$ & $2.25 \mathrm{E}-02$ & $2.23 \mathrm{E}-02$ & $1.41 \mathrm{E}-02$ & $1.41 \mathrm{E}-02$ & $1.42 \mathrm{E}-02$ & $1.41 \mathrm{E}-02$ & $2.22 \mathrm{E}-02$ & $2.23 \mathrm{E}-02$ & $2.22 \mathrm{E}-02$ & $2.24 \mathrm{E}-02$ \\
\hline NO2 & $1.11 \mathrm{E}-01$ & $1.11 \mathrm{E}-01$ & $1.14 \mathrm{E}-01$ & 7.44E-01 & $7.49 \mathrm{E}-01$ & $1.02 \mathrm{E}-01$ & $1.10 \mathrm{E}-01$ & 7.47E-01 & $7.45 \mathrm{E}-01$ & $7.43 \mathrm{E}-01$ & $7.41 \mathrm{E}-01$ & $1.12 \mathrm{E}-01$ & $1.13 \mathrm{E}-01$ & $1.12 \mathrm{E}-01$ & $1.06 \mathrm{E}-01$ \\
\hline NO3 & $3.80 \mathrm{E}+00$ & $3.80 \mathrm{E}+00$ & $3.80 \mathrm{E}+00$ & $2.66 \mathrm{E}+00$ & $2.65 \mathrm{E}+00$ & $3.84 \mathrm{E}+00$ & $3.81 \mathrm{E}+00$ & $2.65 \mathrm{E}+00$ & $2.65 \mathrm{E}+00$ & $2.67 \mathrm{E}+00$ & $2.67 \mathrm{E}+00$ & $3.79 \mathrm{E}+00$ & $3.79 \mathrm{E}+00$ & $3.79 \mathrm{E}+00$ & $3.82 \mathrm{E}+00$ \\
\hline$\overline{\mathrm{OH}}$ & $9.63 \mathrm{E}-01$ & $9.65 \mathrm{E}-01$ & $9.47 \mathrm{E}-01$ & $1.85 \mathrm{E}+00$ & $1.86 \mathrm{E}+00$ & $9.14 \mathrm{E}-01$ & $9.42 \mathrm{E}-01$ & $1.89 \mathrm{E}+00$ & $1.87 \mathrm{E}+00$ & $1.85 \mathrm{E}+00$ & $1.85 \mathrm{E}+00$ & $9.80 \mathrm{E}-01$ & $9.90 \mathrm{E}-01$ & $9.39 \mathrm{E}-01$ & $9.27 \mathrm{E}-01$ \\
\hline PO4 & $1.42 \mathrm{E}-03$ & $1.42 \mathrm{E}-03$ & $1.46 \mathrm{E}-03$ & $1.57 \mathrm{E}-02$ & $1.57 \mathrm{E}-02$ & $1.34 \mathrm{E}-03$ & & $1.57 \mathrm{E}-02$ & $1.56 \mathrm{E}-02$ & $1.57 \mathrm{E}-02$ & $1.57 \mathrm{E}-02$ & $1.37 \mathrm{E}-03$ & $1.38 \mathrm{E}-03$ & $1.41 \mathrm{E}-03$ & $1.34 \mathrm{E}-03$ \\
\hline SO4 & $3.25 \mathrm{E}-01$ & $3.25 \mathrm{E}-01$ & $3.25 \mathrm{E}-01$ & $1.84 \mathrm{E}-01$ & $1.83 \mathrm{E}-01$ & $3.29 \mathrm{E}-01$ & $3.26 \mathrm{E}-01$ & $1.83 \mathrm{E}-01$ & $1.83 \mathrm{E}-01$ & $1.85 \mathrm{E}-01$ & $1.83 \mathrm{E}-01$ & $3.24 \mathrm{E}-01$ & $3.25 \mathrm{E}-01$ & $3.24 \mathrm{E}-01$ & $3.27 \mathrm{E}-01$ \\
\hline$\overline{\mathbf{A g}}$ & $6.13 \mathrm{E}-09$ & $3.60 \mathrm{E}-08$ & $2.65 \mathrm{E}-08$ & $1.33 \mathrm{E}-08$ & $1.14 \mathrm{E}-08$ & $1.25 \mathrm{E}-08$ & $1.24 \mathrm{E}-08$ & $1.14 \mathrm{E}-08$ & $1.14 \mathrm{E}-08$ & $1.46 \mathrm{E}-08$ & $1.15 \mathrm{E}-08$ & $1.24 \mathrm{E}-08$ & $1.24 \mathrm{E}-08$ & $1.23 \mathrm{E}-08$ & $1.24 \mathrm{E}-08$ \\
\hline As & $1.08 \mathrm{E}-06$ & $9.79 \mathrm{E}-07$ & $1.27 \mathrm{E}-06$ & $1.73 \mathrm{E}-06$ & $1.80 \mathrm{E}-05$ & $8.63 \mathrm{E}-06$ & $2.21 \mathrm{E}-05$ & $3.13 \mathrm{E}-05$ & $2.25 \mathrm{E}-05$ & $1.19 \mathrm{E}-06$ & $1.48 \mathrm{E}-05$ & $3.75 \mathrm{E}-05$ & $4.11 \mathrm{E}-05$ & $2.26 \mathrm{E}-05$ & $1.56 \mathrm{E}-05$ \\
\hline $\mathbf{B a}$ & $1.07 \mathrm{E}-09$ & $1.07 \mathrm{E}-08$ & $8.02 \mathrm{E}-09$ & $6.97 \mathrm{E}-08$ & --- & - & --- & 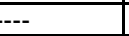 & $-{ }_{-1}$ & $2.50 \mathrm{E}-09$ & --- & --- & 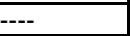 & 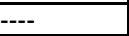 & --- \\
\hline $\mathrm{Ca}$ & $5.05 \mathrm{E}-05$ & $5.04 \mathrm{E}-05$ & $5.05 \mathrm{E}-05$ & E-05 & $3.09 \mathrm{E}-05$ & $5.15 \mathrm{E}-05$ & 5.06 & $3.09 \mathrm{E}-05$ & $3.09 \mathrm{E}-05$ & -05 & E-01 & $5.04 \mathrm{E}-05$ & $5.04 \mathrm{E}-05$ & $5.03 \mathrm{E}-05$ & $5.08 \mathrm{E}-05$ \\
\hline Cd & $2.94 \mathrm{E}-08$ & $2.81 \mathrm{E}-08$ & $3.72 \mathrm{E}-08$ & $4.56 \mathrm{E}-08$ & $4.85 \mathrm{E}-07$ & 1.03E-07 & $6.26 \mathrm{E}-07$ & $9.98 \mathrm{E}-07$ & $6.59 \mathrm{E}-07$ & $2.44 \mathrm{E}-08$ & $3.62 \mathrm{E}-07$ & $1.22 \mathrm{E}-06$ & $1.36 \mathrm{E}-06$ & $6.47 \mathrm{E}-07$ & $3.72 \mathrm{E}-07$ \\
\hline $\mathbf{C l}$ & $4.27 \mathrm{E}-02$ & $4.27 \mathrm{E}-02$ & $4.27 \mathrm{E}-02$ & $3.86 \mathrm{E}-02$ & $3.83 \mathrm{E}-02$ & $4.30 \mathrm{E}-02$ & $4.27 \mathrm{E}-02$ & $3.84 \mathrm{E}-02$ & $3.83 \mathrm{E}-02$ & $3.87 \mathrm{E}-02$ & $3.84 \mathrm{E}-02$ & $4.26 \mathrm{E}-02$ & $4.27 \mathrm{E}-02$ & $4.26 \mathrm{E}-02$ & $4.28 \mathrm{E}-02$ \\
\hline Co & -07 & E-07 & 1.32E-07 & $E-08$ & -07 & $E-08$ & & -07 & $E-07$ & $E-08$ & E-08 & E-07 & E-07 & E-07 & $9.49 \mathrm{E}-08$ \\
\hline $\mathrm{Cr}$ & $6.50 \mathrm{E}-06$ & $3.95 \mathrm{E}-06$ & $1.31 \mathrm{E}-05$ & $5.35 \mathrm{E}-05$ & $7.19 \mathrm{E}-05$ & $8.77 \mathrm{E}-05$ & $7.5 \mathrm{~s}$ & $6.12 \mathrm{E}-05$ & $6.83 \mathrm{E}-05$ & $6.72 \mathrm{E}-05$ & $7.48 \mathrm{E}-05$ & $6.31 \mathrm{E}-05$ & $6.03 \mathrm{E}-05$ & $7.51 \mathrm{E}-05$ & $8.15 \mathrm{E}-05$ \\
\hline $\mathrm{Cu}$ & $5.62 \mathrm{E}-05$ & $5.82 \mathrm{E}-05$ & $4.25 \mathrm{E}-05$ & $1.01 \mathrm{E}-05$ & $2.88 \mathrm{E}-05$ & $6.17 \mathrm{E}-06$ & $=-05$ & $5.91 \mathrm{E}-05$ & $3.91 \mathrm{E}-05$ & $8.57 \mathrm{E}-06$ & $2.15 \mathrm{E}-05$ & $7.23 \mathrm{E}-05$ & $8.05 \mathrm{E}-05$ & $3.84 \mathrm{E}-05$ & $2.21 \mathrm{E}-05$ \\
\hline $\mathrm{Fe}$ & $4.73 \mathrm{E}-07$ & $4.73 \mathrm{E}-07$ & $3.68 \mathrm{E}-07$ & $2.82 \mathrm{E}-07$ & $1.31 \mathrm{E}-06$ & $3.16 \mathrm{E}-07$ & E-06 & $2.65 \mathrm{E}-06$ & $1.76 \mathrm{E}-06$ & $6.51 \mathrm{E}-07$ & $9.88 \mathrm{E}-07$ & $3.24 \mathrm{E}-06$ & $3.59 \mathrm{E}-06$ & $1.74 \mathrm{E}-06$ & $1.02 \mathrm{E}-06$ \\
\hline $\bar{F}$ & $7.12 \mathrm{E}-02$ & $7.11 \mathrm{E}-02$ & $7.12 \mathrm{E}-02$ & $3.62 \mathrm{E}-02$ & $3.60 \mathrm{E}-02$ & & & E-02 & $3.60 \mathrm{E}-02$ & E-02 & $3.60 \mathrm{E}-02$ & $7.11 \mathrm{E}-02$ & $7.11 \mathrm{E}-02$ & E-02 & $7.15 \mathrm{E}-02$ \\
\hline $\mathrm{Hg}$ & $66 \mathrm{E}-07$ & -07 & E-07 & E-07 & $4.16 \mathrm{E}-06$ & $4.32 \mathrm{E}-06$ & & $=-06$ & $4.25 \mathrm{E}-06$ & E-07 & E-06 & E-06 & $4.94 \mathrm{E}-06$ & $4.55 \mathrm{E}-06$ & 4.44E-06 \\
\hline $\mathrm{K}$ & $5.74 \mathrm{E}-03$ & $5.81 \mathrm{E}-03$ & $5.74 \mathrm{E}-03$ & $4.74 \mathrm{E}-03$ & $4.94 \mathrm{E}-03$ & $\mathrm{E}-03$ & -03 & $5.57 \mathrm{E}-03$ & $5.01 \mathrm{E}-03$ & $6 \mathrm{E}-03$ & $4.68 \mathrm{E}-03$ & $5.95 \mathrm{E}-03$ & $6.10 \mathrm{E}-03$ & $5.40 \mathrm{E}-03$ & $5.20 \mathrm{E}-03$ \\
\hline$\overline{M g}$ & --- & --- & $3.45 \mathrm{E}-09$ & $4.40 \mathrm{E}-08$ & $1.77 \mathrm{E}-08$ & $5.43 \mathrm{E}-08$ & $1.11 \mathrm{E}-08$ & $-\cdots$ & $3.51 \mathrm{E}-09$ & $4.63 \mathrm{E}-08$ & $2.79 \mathrm{E}-08$ & --- & --- & $9.03 \mathrm{E}-09$ & $3.19 \mathrm{E}-08$ \\
\hline$M n$ & $2.01 \mathrm{E}-08$ & $2.40 \mathrm{E}-08$ & $3.21 \mathrm{E}-08$ & $3.73 \mathrm{E}-08$ & $6.77 \mathrm{E}-08$ & $3.51 \mathrm{E}-08$ & $8.26 \mathrm{E}-08$ & $1.15 \mathrm{E}-07$ & $8.36 \mathrm{E}-08$ & $2.00 \mathrm{E}-08$ & $5.66 \mathrm{E}-08$ & 1.37E-07 & $1.49 \mathrm{E}-07$ & $8.44 \mathrm{E}-08$ & $5.95 \mathrm{E}-08$ \\
\hline Nd & --- & --- & - & $1.46 \mathrm{E}-06$ & -- & E-06 & --- & - & - & $E-06$ & E-07 & --- & ---- & - & $6.81 \mathrm{E}-07$ \\
\hline$\overline{\mathrm{Ni}}$ & $3.75 \mathrm{E}-07$ & 3.84E-07 & $3.09 \mathrm{E}-07$ & $1.45 \mathrm{E}-07$ & $2.34 \mathrm{E}-07$ & 1.34E-07 & $2.83 \mathrm{E}-07$ & $3.81 \mathrm{E}-07$ & $2.84 \mathrm{E}-07$ & $1.38 \mathrm{E}-07$ & $2.00 \mathrm{E}-07$ & $4.52 \mathrm{E}-07$ & $4.92 \mathrm{E}-07$ & $2.88 \mathrm{E}-07$ & $2.11 \mathrm{E}-07$ \\
\hline $\mathrm{Pb}$ & $1.03 \mathrm{E}-06$ & $8.50 \mathrm{E}-07$ & $1.17 \mathrm{E}-06$ & $1.68 \mathrm{E}-06$ & -- & $-\overline{-}_{-1}$ & 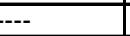 & -- & --- & $9.93 \mathrm{E}-07$ & --- & --- & --- & --- & -- \\
\hline $\mathbf{R u}$ & $5.94 \mathrm{E}-06$ & $6.12 \mathrm{E}-06$ & $4.60 \mathrm{E}-06$ & $1.40 \mathrm{E}-06$ & $3.22 \mathrm{E}-06$ & $1.06 \mathrm{E}-06$ & $4.07 \mathrm{E}-06$ & $6.18 \mathrm{E}-06$ & $4.23 \mathrm{E}-06$ & $1.26 \mathrm{E}-06$ & $2.51 \mathrm{E}-06$ & $7.51 \mathrm{E}-06$ & $8.30 \mathrm{E}-06$ & $4.19 \mathrm{E}-06$ & $2.61 \mathrm{E}-06$ \\
\hline Se & $7.63 \mathrm{E}-06$ & $5.85 \mathrm{E}-06$ & $9.85 \mathrm{E}-06$ & $2.03 \mathrm{E}-05$ & $5.53 \mathrm{E}-05$ & $7.32 \mathrm{E}-05$ & $5.70 \mathrm{E}-05$ & $4.00 \mathrm{E}-05$ & $5.01 \mathrm{E}-05$ & $1.96 \mathrm{E}-05$ & $5.91 \mathrm{E}-05$ & $3.90 \mathrm{E}-05$ & $3.50 \mathrm{E}-05$ & $5.60 \mathrm{E}-05$ & $6.47 \mathrm{E}-05$ \\
\hline$\overline{\mathrm{Si}}$ & $3.64 \mathrm{E}-05$ & $3.19 \mathrm{E}-05$ & $1.86 \mathrm{E}-04$ & $6.09 \mathrm{E}-06$ & -- & -.- & $-\cdots$ & -.- & \begin{tabular}{l|l}
-- & \\
\end{tabular} & $4.76 \mathrm{E}-05$ & -- & --- & - & --- & - \\
\hline $\mathrm{Sr}$ & --- & --- & \begin{tabular}{l|l}
-- & \\
\end{tabular} & $9.83 \mathrm{E}-07$ & -- & $1.36 \mathrm{E}-06$ & -- & -- & -- & $1.08 \mathrm{E}-06$ & $2.71 \mathrm{E}-07$ & --- & --- & --- & $3.67 \mathrm{E}-07$ \\
\hline $\mathbf{Z n}$ & $6.83 \mathrm{E}-05$ & $7.04 \mathrm{E}-05$ & $5.34 \mathrm{E}-05$ & $1.76 \mathrm{E}-05$ & $\begin{array}{l}3.79 \mathrm{E}-05 \\
\end{array}$ & $1.40 \mathrm{E}-05$ & $4.75 \mathrm{E}-05$ & $7.09 \mathrm{E}-05$ & $4.91 \mathrm{E}-05$ & $1.60 \mathrm{E}-05$ & $3.00 \mathrm{E}-05$ & $8.58 \mathrm{E}-05$ & $9.46 \mathrm{E}-05$ & $4.89 \mathrm{E}-05$ & $3.12 \mathrm{E}-05$ \\
\hline$Z \mathbf{Z r}$ & $1.80 \mathrm{E}-06$ & $1.86 \mathrm{E}-06$ & $1.37 \mathrm{E}-06$ & $3.56 \mathrm{E}-07$ & $9.39 \mathrm{E}-07$ & $2.39 \mathrm{E}-07$ & $1.20 \mathrm{E}-06$ & $1.88 \mathrm{E}-06$ & $1.26 \mathrm{E}-06$ & $3.10 \mathrm{E}-07$ & $7.12 \mathrm{E}-07$ & $2.30 \mathrm{E}-06$ & $2.55 \mathrm{E}-06$ & $1.24 \mathrm{E}-06$ & $7.35 \mathrm{E}-07$ \\
\hline Insol.Sol.mg/L & 0 & 0 & 0 & 0 & 0 & 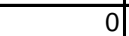 & 0 & 0 & 0 & 0 & 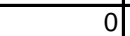 & & & & \\
\hline
\end{tabular}


Table 15: Projected Feed Composition After Dissolution and Dilution to 6.44 M Total Sodium Concentration no sludge ; $10 \%$ of interstitial supernate remaining (continued)

\begin{tabular}{|c|c|c|c|c|c|c|c|c|c|c|c|c|c|c|c|}
\hline \multicolumn{16}{|c|}{ Radionuclide Composition (Ci/gal) } \\
\hline & \begin{tabular}{|l|} 
Tank 25 \\
\end{tabular} & Tank 27 & Tank 28 & Tank 29 & Tank 31 & \begin{tabular}{|l|} 
Tank 33 \\
\end{tabular} & Tank 34 & \begin{tabular}{|l|} 
Tank 36 \\
\end{tabular} & Tank 37 & Tank 38 & \begin{tabular}{|l|} 
Tank 41 \\
\end{tabular} & Tank 44 & Tank 45 & Tank 46 & Tank 47 \\
\hline C-14 & \begin{tabular}{|l|}
--- \\
\end{tabular} & ---- & \begin{tabular}{|l|}
--- \\
\end{tabular} & \begin{tabular}{|l|}
--- \\
\end{tabular} & \begin{tabular}{|l|}
--- \\
\end{tabular} & \begin{tabular}{|l|}
--- \\
\end{tabular} & 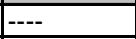 & $-{ }_{---}$ & $-{ }_{---}$ & ---- & ---- & ---- & 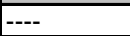 & $-\ldots$ & $-\ldots$ \\
\hline $\mathrm{Ni}-59$ & --- & --- & $-\cdots$ & --- & --- & ---- & --- & --- & ---- & --- & --- & --- & --- & --- & --- \\
\hline $\mathrm{Ni}-63$ & --- & --- & --- & --- & --- & ---- & --- & --- & --- & --- & --- & --- & --- & --- & --- \\
\hline Co-60 & ---- & --- & ---- & ---- & ---- & --- & --- & --- & --- & ---- & --- & ---- & --- & --- & ---- \\
\hline Se-79 & $-\cdots$ & -- & --- & --- & --- & --- & --- & --- & --- & --- & $-{ }_{---}$ & --- & - & -- & --- \\
\hline TC-99 & $-\cdots$ & --- & --- & --- & --- & --- & --- & --- & --- & --- & $-{ }_{---}$ & --- & - & --- & --- \\
\hline Ru-106 & --- & --- & --- & -- & - & --- & --- & --- & --- & --- & -- & --- & - & --- & --- \\
\hline Rh-106 & --- & --- & --- & --- & - & --- & --- & --- & --- & --- & --- & - & - & --- & --- \\
\hline Sb-125 & --- & --- & --- & --- & --- & --- & --- & --- & --- & --- & --- & - & - & --- & --- \\
\hline Sn-126 & --- & --- & --- & ---- & --- & --- & --- & --- & --- & --- & --- & --- & - & --- & ---- \\
\hline I-129 & $-{ }_{-}$ & --- & $-{ }_{-1}$ & $-{ }_{---}$ & - & --- & --- & --- & --- & $-{ }_{---}$ & $-{ }_{---}$ & \begin{tabular}{|l}
--- \\
\end{tabular} & - & --- & --- \\
\hline Cs-134 & $-\cdots$ & ---- & $-\cdots-$ & $-\cdots$ & $-\cdots-$ & ---- & ---- & $-\cdots$ & ---- & ---- & --- & $-\cdots$ & $-\cdots$ & ---- & \begin{tabular}{|c|}
--- \\
\end{tabular} \\
\hline Cs-135 & --- & ---- & --- & ---- & \begin{tabular}{|l|}
--- \\
\end{tabular} & --- & --- & $-\cdots$ & ---- & ---- & $-\mathrm{-}^{--}$ & $-{ }_{---}$ & --- & ---- & --- \\
\hline Cs-137 & $3.15 \mathrm{E}-02$ & $2.99 \mathrm{E}-02$ & $3.28 \mathrm{E}-02$ & $2.80 \mathrm{E}-02$ & \begin{tabular}{|l}
$7.70 \mathrm{E}-02$ \\
\end{tabular} & \begin{tabular}{|c|}
$7.37 \mathrm{E}-04$ \\
\end{tabular} & 2.56E-02 & 1.42E-01 & \begin{tabular}{|c|}
$1.03 \mathrm{E}-01$ \\
\end{tabular} & $1.86 \mathrm{E}-03$ & \begin{tabular}{|c|}
$1.72 \mathrm{E}-02$ \\
\end{tabular} & $3.88 \mathrm{E}-02$ & $3.68 \mathrm{E}-02$ & 4.74E-02 & $1.97 \mathrm{E}-02$ \\
\hline$B a-137 m$ & $2.98 \mathrm{E}-02$ & $2.83 \mathrm{E}-02$ & $3.10 \mathrm{E}-02$ & $2.65 \mathrm{E}-02$ & $7.29 \mathrm{E}-02$ & $6.97 \mathrm{E}-04$ & $2.42 \mathrm{E}-02$ & $1.34 \mathrm{E}-01$ & $9.74 \mathrm{E}-02$ & $1.76 \mathrm{E}-03$ & $1.62 \mathrm{E}-02$ & $3.67 \mathrm{E}-02$ & $3.48 \mathrm{E}-02$ & $4.48 \mathrm{E}-02$ & $1.86 \mathrm{E}-02$ \\
\hline Sr-90 & $2.13 \mathrm{E}-05$ & $2.13 \mathrm{E}-05$ & $2.13 \mathrm{E}-05$ & $7.52 \mathrm{E}-05$ & $7.46 \mathrm{E}-05$ & $8.16 \mathrm{E}-05$ & $8.09 \mathrm{E}-05$ & $7.46 \mathrm{E}-05$ & $7.46 \mathrm{E}-05$ & $1.99 \mathrm{E}-05$ & $1.97 \mathrm{E}-05$ & $2.12 \mathrm{E}-05$ & $2.13 \mathrm{E}-05$ & $2.12 \mathrm{E}-05$ & $2.14 \mathrm{E}-05$ \\
\hline $\mathrm{Y}-90$ & $2.13 \mathrm{E}-05$ & $2.13 \mathrm{E}-05$ & $2.13 \mathrm{E}-05$ & $7.52 \mathrm{E}-05$ & \begin{tabular}{|l}
$7.46 \mathrm{E}-05$ \\
\end{tabular} & $8.16 \mathrm{E}-05$ & $8.09 \mathrm{E}-05$ & $7.46 \mathrm{E}-05$ & $7.46 \mathrm{E}-05$ & $1.99 \mathrm{E}-05$ & 1.97E-05 & 2.12E-05 & $2.13 \mathrm{E}-05$ & $2.12 \mathrm{E}-05$ & $2.14 \mathrm{E}-05$ \\
\hline $\mathrm{H}-3$ & \begin{tabular}{|l|}
---- \\
\end{tabular} & \begin{tabular}{|l|}
---- \\
\end{tabular} & \begin{tabular}{|l|}
--- \\
\end{tabular} & \begin{tabular}{|l|}
---- \\
\end{tabular} & \begin{tabular}{|l|}
--- \\
\end{tabular} & \begin{tabular}{|l|}
---- \\
\end{tabular} & ---- & \begin{tabular}{|l|}
--- \\
\end{tabular} & ---- & $\mid---$ & ---- & ---- & ---- & ---- & ---- \\
\hline Ce-144 & --- & --- & ---- & --- & --- & --- & --- & --- & ---- & --- & --- & --- & - & --- & --- \\
\hline Pr-144 & $-{ }_{-1}$ & --- & --- & $-{ }_{-1}$ & --- & --- & $-{ }_{---}$ & --- & ---- & --- & $-{ }_{---}$ & $-{ }_{---}$ & --- & --- & --- \\
\hline Pm-147 & --- & --- & --- & --- & --- & --- & --- & --- & --- & --- & -- & - & - & --- & --- \\
\hline Eu-154 & --- & --- & --- & ---- & \begin{tabular}{|l|}
--- \\
\end{tabular} & $-{ }_{---}$ & --- & \begin{tabular}{|l|}
--- \\
\end{tabular} & \begin{tabular}{|l|}
---- \\
\end{tabular} & --- & $-{ }_{---}$ & - & - & --- & --- \\
\hline Th-232 & --- & --- & \begin{tabular}{|l|}
--- \\
\end{tabular} & $3.68 \mathrm{E}-12$ & $3.65 \mathrm{E}-12$ & --- & --- & \begin{tabular}{|c|}
$3.65 \mathrm{E}-12$ \\
\end{tabular} & $3.65 \mathrm{E}-12$ & --- & --- & \begin{tabular}{|l|}
--- \\
\end{tabular} & --- & \begin{tabular}{|l|}
--- \\
\end{tabular} & --- \\
\hline $\mathrm{U}-232$ & \begin{tabular}{|c|}
$2.56 \mathrm{E}-11$ \\
\end{tabular} & $2.55 \mathrm{E}-11$ & $2.56 \mathrm{E}-11$ & $1.05 \mathrm{E}-12$ & $1.04 \mathrm{E}-12$ & $3.41 \mathrm{E}-10$ & $3.43 \mathrm{E}-10$ & $1.04 \mathrm{E}-12$ & $1.04 \mathrm{E}-12$ & --- & --- & $2.55 \mathrm{E}-11$ & 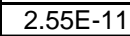 & $2.55 \mathrm{E}-11$ & $2.57 \mathrm{E}-11$ \\
\hline U-233 & \begin{tabular}{|l|}
--- \\
\end{tabular} & $\mid---$ & $\mid---$ & $7.55 \mathrm{E}-10$ & $7.49 \mathrm{E}-10$ & \begin{tabular}{|l|}
---- \\
\end{tabular} & $\mid---$ & $7.49 \mathrm{E}-10$ & $7.49 \mathrm{E}-10$ & 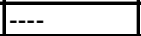 & $-{ }_{---}$ & $\mid---$ & \begin{tabular}{|l|}
--- \\
\end{tabular} & --- & ---- \\
\hline U-234 & --- & $-\cdots$ & $-{ }_{---}$ & $1.07 \mathrm{E}-10$ & $1.06 \mathrm{E}-10$ & --- & $\begin{array}{ll}--- \\
\end{array}$ & $1.06 \mathrm{E}-10$ & $1.06 \mathrm{E}-10$ & 1.75E-09 & \begin{tabular}{|c|}
$1.73 E-09$ \\
\end{tabular} & \begin{tabular}{|l|}
--- \\
\end{tabular} & \begin{tabular}{|l|}
--- \\
\end{tabular} & ---- & \begin{tabular}{|l|}
--- \\
\end{tabular} \\
\hline U-235 & $5.72 \mathrm{E}-11$ & $5.72 \mathrm{E}-11$ & 5.72E-11 & $2.91 \mathrm{E}-12$ & $2.89 \mathrm{E}-12$ & $4.04 \mathrm{E}-10$ & $3.21 \mathrm{E}-10$ & $2.89 \mathrm{E}-12$ & $2.89 \mathrm{E}-12$ & $1.76 \mathrm{E}-11$ & $1.74 \mathrm{E}-11$ & $5.71 \mathrm{E}-11$ & $5.71 \mathrm{E}-11$ & $5.70 \mathrm{E}-11$ & $5.74 \mathrm{E}-11$ \\
\hline U-236 & $\begin{array}{ll}--- \\
\end{array}$ & $\mid---$ & $\mid---$ & $1.10 \mathrm{E}-11$ & $1.09 \mathrm{E}-11$ & \begin{tabular}{|l|}
---- \\
\end{tabular} & \begin{tabular}{|l|}
--- \\
\end{tabular} & $1.09 \mathrm{E}-11$ & $1.09 \mathrm{E}-11$ & $2.66 \mathrm{E}-10$ & $2.64 \mathrm{E}-10$ & $\mid----$ & ---- & $\mid---$ & $\mid----$ \\
\hline $\mathrm{U}-238$ & $5.23 \mathrm{E}-09$ & 5.22E-09 & 5.22E-09 & $3.41 \mathrm{E}-11$ & $3.38 \mathrm{E}-11$ & $3.69 \mathrm{E}-08$ & 2.27E-08 & $3.38 \mathrm{E}-11$ & $3.38 \mathrm{E}-11$ & $2.47 \mathrm{E}-12$ & $2.45 \mathrm{E}-12$ & $5.22 \mathrm{E}-09$ & $5.22 \mathrm{E}-09$ & $5.21 \mathrm{E}-09$ & $5.25 \mathrm{E}-09$ \\
\hline Np-237 & \begin{tabular}{|l|}
--- \\
\end{tabular} & $\mid---$ & \begin{tabular}{|l|}
--- \\
\end{tabular} & $2.74 \mathrm{E}-10$ & $2.72 \mathrm{E}-10$ & $3.04 \mathrm{E}-08$ & $1.75 \mathrm{E}-08$ & $2.72 \mathrm{E}-10$ & $2.72 \mathrm{E}-10$ & $1.52 \mathrm{E}-09$ & $1.51 \mathrm{E}-09$ & \begin{tabular}{|l|}
--- \\
\end{tabular} & \begin{tabular}{|l}
--- \\
\end{tabular} & \begin{tabular}{|l|}
--- \\
\end{tabular} & ---- \\
\hline Pu-238 & $4.33 \mathrm{E}-05$ & $4.33 \mathrm{E}-05$ & \begin{tabular}{|l}
$4.33 \mathrm{E}-05$ \\
\end{tabular} & $1.05 \mathrm{E}-06$ & $1.04 \mathrm{E}-06$ & - & $\mid---$ & $1.04 \mathrm{E}-06$ & $1.04 \mathrm{E}-06$ & $4.81 \mathrm{E}-05$ & $4.76 \mathrm{E}-05$ & $4.32 \mathrm{E}-05$ & $4.33 \mathrm{E}-05$ & \begin{tabular}{|l|}
$4.32 \mathrm{E}-05$ \\
\end{tabular} & $4.35 \mathrm{E}-05$ \\
\hline Pu-239 & $6.19 \mathrm{E}-06$ & $6.18 \mathrm{E}-06$ & $6.19 \mathrm{E}-06$ & $2.71 \mathrm{E}-08$ & $2.69 \mathrm{E}-08$ & $4.42 \mathrm{E}-06$ & $3.66 \mathrm{E}-06$ & $2.69 \mathrm{E}-08$ & $2.69 \mathrm{E}-08$ & $1.48 \mathrm{E}-07$ & $1.47 \mathrm{E}-07$ & $6.17 \mathrm{E}-06$ & $6.18 \mathrm{E}-06$ & $6.17 \mathrm{E}-06$ & $6.21 \mathrm{E}-06$ \\
\hline Pu-240 & $1.38 \mathrm{E}-06$ & $1.38 \mathrm{E}-06$ & $1.38 \mathrm{E}-06$ & $1.12 \mathrm{E}-08$ & $1.11 \mathrm{E}-08$ & 9.87E-07 & $8.18 \mathrm{E}-07$ & $1.11 \mathrm{E}-08$ & $1.11 \mathrm{E}-08$ & $9.79 \mathrm{E}-08$ & $9.70 \mathrm{E}-08$ & $1.38 \mathrm{E}-06$ & $1.38 \mathrm{E}-06$ & $1.38 \mathrm{E}-06$ & $1.39 \mathrm{E}-06$ \\
\hline Pu-241 & $3.71 \mathrm{E}-05$ & $3.71 \mathrm{E}-05$ & $3.71 \mathrm{E}-05$ & $2.43 \mathrm{E}-07$ & $2.41 \mathrm{E}-07$ & $2.57 \mathrm{E}-05$ & $2.08 \mathrm{E}-05$ & $2.41 \mathrm{E}-07$ & $2.41 \mathrm{E}-07$ & $8.18 \mathrm{E}-06$ & $8.11 \mathrm{E}-06$ & $3.70 \mathrm{E}-05$ & $3.71 \mathrm{E}-05$ & $3.70 \mathrm{E}-05$ & $3.73 \mathrm{E}-05$ \\
\hline Pu-242 & $2.85 \mathrm{E}-10$ & $2.84 \mathrm{E}-10$ & 2.84E-10 & $7.44 \mathrm{E}-12$ & $7.38 \mathrm{E}-12$ & $2.03 \mathrm{E}-10$ & $1.70 \mathrm{E}-10$ & $7.38 \mathrm{E}-12$ & $7.38 \mathrm{E}-12$ & $1.84 \mathrm{E}-09$ & $1.82 \mathrm{E}-09$ & $2.84 \mathrm{E}-10$ & $2.84 \mathrm{E}-10$ & $2.84 \mathrm{E}-10$ & $2.86 \mathrm{E}-10$ \\
\hline Am-241 & \begin{tabular}{|l|}
--- \\
\end{tabular} & $\mid---$ & $\mid---$ & $3.08 \mathrm{E}-07$ & $3.05 \mathrm{E}-07$ & $4.63 \mathrm{E}-05$ & $4.69 \mathrm{E}-05$ & $3.05 \mathrm{E}-07$ & $3.05 \mathrm{E}-07$ & $2.56 \mathrm{E}-07$ & $2.54 \mathrm{E}-07$ & \begin{tabular}{|l|}
--- \\
\end{tabular} & $\begin{array}{ll}--- \\
\end{array}$ & \begin{tabular}{|l|}
--- \\
\end{tabular} & ---- \\
\hline$A m-242 m$ & --- & --- & $-{ }_{---}$ & $3.76 \mathrm{E}-10$ & $3.73 \mathrm{E}-10$ & $6.32 \mathrm{E}-08$ & $6.39 \mathrm{E}-08$ & $3.73 \mathrm{E}-10$ & $3.73 \mathrm{E}-10$ & $3.19 \mathrm{E}-10$ & $3.16 \mathrm{E}-10$ & --- & --- & $-{ }_{-1}$ & - \\
\hline$\overline{C m-242}$ & \begin{tabular}{|l|}
--- \\
\end{tabular} & --- & \begin{tabular}{|l|}
--- \\
\end{tabular} & ---- & \begin{tabular}{|l|}
--- \\
\end{tabular} & \begin{tabular}{|l|}
---- \\
\end{tabular} & \begin{tabular}{|l|}
--- \\
\end{tabular} & $-\infty$ & \begin{tabular}{|l|}
--- \\
\end{tabular} & \begin{tabular}{|l|}
--- \\
\end{tabular} & \begin{tabular}{|l}
--- \\
\end{tabular} & \begin{tabular}{|l|}
--- \\
\end{tabular} & ---- & \begin{tabular}{|l|}
$-\cdots-$ \\
\end{tabular} & \begin{tabular}{|l|}
--- \\
\end{tabular} \\
\hline Cm-244 & $5.37 \mathrm{E}-10$ & 5.37E-10 & 5.37E-10 & $9.02 \mathrm{E}-10$ & $8.95 \mathrm{E}-10$ & $2.45 \mathrm{E}-08$ & $2.40 \mathrm{E}-08$ & $8.95 \mathrm{E}-10$ & $8.95 \mathrm{E}-10$ & 4.33E-09 & $4.29 \mathrm{E}-09$ & $5.36 \mathrm{E}-10$ & $5.37 \mathrm{E}-10$ & $5.36 \mathrm{E}-10$ & $5.40 \mathrm{E}-10$ \\
\hline$\overline{C m-245}$ & $2.12 \mathrm{E}-16$ & $2.12 \mathrm{E}-16$ & $2.12 \mathrm{E}-16$ & $1.11 \mathrm{E}-13$ & $1.10 \mathrm{E}-13$ & $9.66 \mathrm{E}-15$ & $9.81 \mathrm{E}-15$ & $1.10 \mathrm{E}-13$ & $1.10 \mathrm{E}-13$ & $2.67 \mathrm{E}-13$ & $2.64 \mathrm{E}-13$ & $2.11 \mathrm{E}-16$ & $2.12 \mathrm{E}-16$ & $2.11 \mathrm{E}-16$ & $2.13 \mathrm{E}-16$ \\
\hline Total Alpha & $5.09 \mathrm{E}-05$ & $5.08 \mathrm{E}-05$ & 5.09E-05 & $1.39 \mathrm{E}-06$ & $1.38 \mathrm{E}-06$ & $5.18 \mathrm{E}-05$ & $5.15 \mathrm{E}-05$ & $1.38 \mathrm{E}-06$ & $1.38 \mathrm{E}-06$ & $4.86 \mathrm{E}-05$ & $4.81 \mathrm{E}-05$ & $5.08 \mathrm{E}-05$ & $5.08 \mathrm{E}-05$ & $5.07 \mathrm{E}-05$ & $5.11 \mathrm{E}-05$ \\
\hline
\end{tabular}


Table 16: Projected Feed Volumes After Dissolution and Dilution to 6.44 M Total Sodium Concentration: 30\% interstitial supernate remaining

\begin{tabular}{|l|c|c|c|c|c|c|c|c|}
\hline \multicolumn{7}{|c|}{ Volume of Saltcake Components After Dilution To 6.44 M Na } \\
\hline & Tank 25 & Tank 27 & Tank 28 & Tank 29 & Tank 31 & Tank 33 & Tank 34 & Tank 36 \\
\hline Salt Volume (gal) & $1.11 \mathrm{E}+06$ & $4.63 \mathrm{E}+05$ & $1.03 \mathrm{E}+06$ & $1.00 \mathrm{E}+06$ & $1.01 \mathrm{E}+06$ & $2.27 \mathrm{E}+05$ & $2.12 \mathrm{E}+05$ & $1.09 \mathrm{E}+06$ \\
\hline Salt Mass (Ib) & $1.34 \mathrm{E}+07$ & $5.60 \mathrm{E}+06$ & $1.25 \mathrm{E}+07$ & $1.21 \mathrm{E}+07$ & $1.23 \mathrm{E}+07$ & $2.75 \mathrm{E}+06$ & $2.56 \mathrm{E}+06$ & $1.32 \mathrm{E}+07$ \\
\hline Salt Volume (L) & $4.19 \mathrm{E}+06$ & $1.75 \mathrm{E}+06$ & $3.91 \mathrm{E}+06$ & $3.79 \mathrm{E}+06$ & $3.84 \mathrm{E}+06$ & $8.59 \mathrm{E}+05$ & $8.03 \mathrm{E}+05$ & $4.14 \mathrm{E}+06$ \\
\hline Intst. Supr. Vol. (L) & $2.77 \mathrm{E}+05$ & $1.16 \mathrm{E}+05$ & $2.58 \mathrm{E}+05$ & $2.50 \mathrm{E}+05$ & $2.53 \mathrm{E}+05$ & $5.67 \mathrm{E}+04$ & $5.30 \mathrm{E}+04$ & $2.73 \mathrm{E}+05$ \\
\hline Dilution Volume (L) & $1.06 \mathrm{E}+07$ & $4.47 \mathrm{E}+06$ & $9.94 \mathrm{E}+06$ & $1.05 \mathrm{E}+07$ & $1.10 \mathrm{E}+07$ & $2.08 \mathrm{E}+06$ & $2.02 \mathrm{E}+06$ & $1.19 \mathrm{E}+07$ \\
\hline Dilution Mass (Ib) & $2.34 \mathrm{E}+07$ & $9.85 \mathrm{E}+06$ & $2.19 \mathrm{E}+07$ & $2.30 \mathrm{E}+07$ & $2.42 \mathrm{E}+07$ & $4.58 \mathrm{E}+06$ & $4.46 \mathrm{E}+06$ & $2.62 \mathrm{E}+07$ \\
\hline Total Volume (L) & $1.29 \mathrm{E}+07$ & $5.41 \mathrm{E}+06$ & $1.20 \mathrm{E}+07$ & $1.23 \mathrm{E}+07$ & $1.28 \mathrm{E}+07$ & $2.57 \mathrm{E}+06$ & $2.46 \mathrm{E}+06$ & $1.38 \mathrm{E}+07$ \\
\hline Total Volume (gal) & $3.41 \mathrm{E}+06$ & $1.43 \mathrm{E}+06$ & $3.18 \mathrm{E}+06$ & $3.25 \mathrm{E}+06$ & $3.38 \mathrm{E}+06$ & $6.78 \mathrm{E}+05$ & $6.50 \mathrm{E}+05$ & $3.64 \mathrm{E}+06$ \\
\hline Total Mass (lb) & $3.68 \mathrm{E}+07$ & $1.54 \mathrm{E}+07$ & $3.44 \mathrm{E}+07$ & $3.51 \mathrm{E}+07$ & $3.65 \mathrm{E}+07$ & $7.33 \mathrm{E}+06$ & $7.02 \mathrm{E}+06$ & $3.94 \mathrm{E}+07$ \\
\hline Solution Sp. G. & $1.30 \mathrm{E}+00$ & $1.30 \mathrm{E}+00$ & $1.30 \mathrm{E}+00$ & $1.30 \mathrm{E}+00$ & $1.30 \mathrm{E}+00$ & $1.30 \mathrm{E}+00$ & $1.30 \mathrm{E}+00$ & $1.30 \mathrm{E}+00$ \\
\hline
\end{tabular}

\begin{tabular}{|l|c|c|c|c|c|c|c|}
\hline \multicolumn{7}{|c|}{ Volume of Saltcake Components After Dilution To 6.44 M Na } \\
\hline & Tank 37 & Tank 38 & Tank 41 & Tank 44 & Tank 45 & Tank 46 & Tank 47 \\
\hline Salt Volume (gal) & $9.73 \mathrm{E}+05$ & $8.86 \mathrm{E}+05$ & $1.23 \mathrm{E}+06$ & $9.89 \mathrm{E}+05$ & $1.13 \mathrm{E}+06$ & $3.19 \mathrm{E}+05$ & $8.68 \mathrm{E}+05$ \\
\hline Salt Mass (Ib) & $1.18 \mathrm{E}+07$ & $1.07 \mathrm{E}+07$ & $1.49 \mathrm{E}+07$ & $1.20 \mathrm{E}+07$ & $1.37 \mathrm{E}+07$ & $3.86 \mathrm{E}+06$ & $1.05 \mathrm{E}+07$ \\
\hline Salt Volume (L) & $3.68 \mathrm{E}+06$ & $3.35 \mathrm{E}+06$ & $4.66 \mathrm{E}+06$ & $3.74 \mathrm{E}+06$ & $4.28 \mathrm{E}+06$ & $1.21 \mathrm{E}+06$ & $3.29 \mathrm{E}+06$ \\
\hline Intst. Supr. Vol. (L) & $2.43 \mathrm{E}+05$ & $2.21 \mathrm{E}+05$ & $3.08 \mathrm{E}+05$ & $2.47 \mathrm{E}+05$ & $2.82 \mathrm{E}+05$ & $7.97 \mathrm{E}+04$ & $2.17 \mathrm{E}+05$ \\
\hline Dilution Volume (L) & $1.06 \mathrm{E}+07$ & $9.12 \mathrm{E}+06$ & $1.32 \mathrm{E}+07$ & $9.61 \mathrm{E}+06$ & $1.09 \mathrm{E}+07$ & $3.11 \mathrm{E}+06$ & $8.19 \mathrm{E}+06$ \\
\hline Dilution Mass (Ib) & $2.33 \mathrm{E}+07$ & $2.01 \mathrm{E}+07$ & $2.91 \mathrm{E}+07$ & $2.12 \mathrm{E}+07$ & $2.41 \mathrm{E}+07$ & $6.86 \mathrm{E}+06$ & $1.80 \mathrm{E}+07$ \\
\hline Total Volume (L) & $1.23 \mathrm{E}+07$ & $1.08 \mathrm{E}+07$ & $1.54 \mathrm{E}+07$ & $1.16 \mathrm{E}+07$ & $1.32 \mathrm{E}+07$ & $3.75 \mathrm{E}+06$ & $1.00 \mathrm{E}+07$ \\
\hline Total Volume (gal) & $3.24 \mathrm{E}+06$ & $2.85 \mathrm{E}+06$ & $4.07 \mathrm{E}+06$ & $3.06 \mathrm{E}+06$ & $3.49 \mathrm{E}+06$ & $9.91 \mathrm{E}+05$ & $2.64 \mathrm{E}+06$ \\
\hline Total Mass (lb) & $3.50 \mathrm{E}+07$ & $3.08 \mathrm{E}+07$ & $4.39 \mathrm{E}+07$ & $3.31 \mathrm{E}+07$ & $3.77 \mathrm{E}+07$ & $1.07 \mathrm{E}+07$ & $2.85 \mathrm{E}+07$ \\
\hline Solution Sp. G. & $1.30 \mathrm{E}+00$ & $1.30 \mathrm{E}+00$ & $1.30 \mathrm{E}+00$ & $1.30 \mathrm{E}+00$ & $1.30 \mathrm{E}+00$ & $1.30 \mathrm{E}+00$ & $1.30 \mathrm{E}+00$ \\
\hline
\end{tabular}


Table 17: Projected Feed Volumes After Dissolution and Dilution to 6.44 M Total Sodium Concentration: 20\% interstitial supernate remaining

\begin{tabular}{|l|c|c|c|c|c|c|c|c|}
\hline \multicolumn{10}{|c|}{$\mathbf{6 0 0} \mathbf{~ m g / L ~ S l u d g e - ~ 2 0 \% ~ I n t e r s t i t i a l ~ S u p e r n a t e ~}$} \\
\hline & Volume of Saltcake Components After Dilution To 6.44 M Na \\
\hline & Tank 25 & Tank 27 & Tank 28 & Tank 29 & Tank 31 & Tank 33 & Tank 34 & Tank 36 \\
\hline Salt Volume (gal) & $1.11 \mathrm{E}+06$ & $4.63 \mathrm{E}+05$ & $1.03 \mathrm{E}+06$ & $1.00 \mathrm{E}+06$ & $1.01 \mathrm{E}+06$ & $2.27 \mathrm{E}+05$ & $2.12 \mathrm{E}+05$ & $1.09 \mathrm{E}+06$ \\
\hline Salt Mass (lb) & $1.34 \mathrm{E}+07$ & $5.60 \mathrm{E}+06$ & $1.25 \mathrm{E}+07$ & $1.21 \mathrm{E}+07$ & $1.23 \mathrm{E}+07$ & $2.75 \mathrm{E}+06$ & $2.56 \mathrm{E}+06$ & $1.32 \mathrm{E}+07$ \\
\hline Salt Volume (L) & $4.19 \mathrm{E}+06$ & $1.75 \mathrm{E}+06$ & $3.91 \mathrm{E}+06$ & $3.79 \mathrm{E}+06$ & $3.84 \mathrm{E}+06$ & $8.59 \mathrm{E}+05$ & $8.03 \mathrm{E}+05$ & $4.14 \mathrm{E}+06$ \\
\hline Intst. Supr. Vol. (L) & $1.85 \mathrm{E}+05$ & $7.71 \mathrm{E}+04$ & $1.72 \mathrm{E}+05$ & $1.67 \mathrm{E}+05$ & $1.69 \mathrm{E}+05$ & $3.78 \mathrm{E}+04$ & $3.53 \mathrm{E}+04$ & $1.82 \mathrm{E}+05$ \\
\hline Dilution Volume (L) & $1.04 \mathrm{E}+07$ & $4.37 \mathrm{E}+06$ & $9.72 \mathrm{E}+06$ & $1.04 \mathrm{E}+07$ & $1.08 \mathrm{E}+07$ & $2.07 \mathrm{E}+06$ & $1.99 \mathrm{E}+06$ & $1.16 \mathrm{E}+07$ \\
\hline Dilution Mass (Ib) & $2.30 \mathrm{E}+07$ & $9.63 \mathrm{E}+06$ & $2.14 \mathrm{E}+07$ & $2.29 \mathrm{E}+07$ & $2.38 \mathrm{E}+07$ & $4.55 \mathrm{E}+06$ & $4.37 \mathrm{E}+06$ & $2.56 \mathrm{E}+07$ \\
\hline Total Volume (L) & $1.27 \mathrm{E}+07$ & $5.33 \mathrm{E}+06$ & $1.19 \mathrm{E}+07$ & $1.22 \mathrm{E}+07$ & $1.26 \mathrm{E}+07$ & $2.56 \mathrm{E}+06$ & $2.43 \mathrm{E}+06$ & $1.36 \mathrm{E}+07$ \\
\hline Total Volume (gal) & $3.36 \mathrm{E}+06$ & $1.41 \mathrm{E}+06$ & $3.14 \mathrm{E}+06$ & $3.23 \mathrm{E}+06$ & $3.33 \mathrm{E}+06$ & $6.75 \mathrm{E}+05$ & $6.42 \mathrm{E}+05$ & $3.60 \mathrm{E}+06$ \\
\hline Total Mass (lb) & $3.64 \mathrm{E}+07$ & $1.52 \mathrm{E}+07$ & $3.39 \mathrm{E}+07$ & $3.50 \mathrm{E}+07$ & $3.60 \mathrm{E}+07$ & $7.30 \mathrm{E}+06$ & $6.94 \mathrm{E}+06$ & $3.89 \mathrm{E}+07$ \\
\hline Solution Sp. G. & $1.30 \mathrm{E}+00$ & $1.30 \mathrm{E}+00$ & $1.30 \mathrm{E}+00$ & $1.30 \mathrm{E}+00$ & $1.30 \mathrm{E}+00$ & $1.30 \mathrm{E}+00$ & $1.30 \mathrm{E}+00$ & $1.30 \mathrm{E}+00$ \\
\hline
\end{tabular}

\begin{tabular}{|l|c|c|c|c|c|c|c|}
\hline \multicolumn{7}{|c|}{ Volume of Saltcake Components After Dilution To 6.44 M Na } \\
\hline & Tank 37 & Tank 38 & Tank 41 & Tank 44 & Tank 45 & Tank 46 & Tank 47 \\
\hline Salt Volume (gal) & $9.73 \mathrm{E}+05$ & $8.86 \mathrm{E}+05$ & $1.23 \mathrm{E}+06$ & $9.89 \mathrm{E}+05$ & $1.13 \mathrm{E}+06$ & $3.19 \mathrm{E}+05$ & $8.68 \mathrm{E}+05$ \\
\hline Salt Mass (lb) & $1.18 \mathrm{E}+07$ & $1.07 \mathrm{E}+07$ & $1.49 \mathrm{E}+07$ & $1.20 \mathrm{E}+07$ & $1.37 \mathrm{E}+07$ & $3.86 \mathrm{E}+06$ & $1.05 \mathrm{E}+07$ \\
\hline Salt Volume (L) & $3.68 \mathrm{E}+06$ & $3.35 \mathrm{E}+06$ & $4.66 \mathrm{E}+06$ & $3.74 \mathrm{E}+06$ & $4.28 \mathrm{E}+06$ & $1.21 \mathrm{E}+06$ & $3.29 \mathrm{E}+06$ \\
\hline Intst. Supr. Vol. (L) & $1.62 \mathrm{E}+05$ & $1.48 \mathrm{E}+05$ & $2.05 \mathrm{E}+05$ & $1.65 \mathrm{E}+05$ & $1.88 \mathrm{E}+05$ & $5.31 \mathrm{E}+04$ & $1.45 \mathrm{E}+05$ \\
\hline Dilution Volume (L) & $1.04 \mathrm{E}+07$ & $9.10 \mathrm{E}+06$ & $1.30 \mathrm{E}+07$ & $9.37 \mathrm{E}+06$ & $1.07 \mathrm{E}+07$ & $3.03 \mathrm{E}+06$ & $8.06 \mathrm{E}+06$ \\
\hline Dilution Mass (lb) & $2.28 \mathrm{E}+07$ & $2.00 \mathrm{E}+07$ & $2.86 \mathrm{E}+07$ & $2.06 \mathrm{E}+07$ & $2.35 \mathrm{E}+07$ & $6.68 \mathrm{E}+06$ & $1.78 \mathrm{E}+07$ \\
\hline Total Volume (L) & $1.21 \mathrm{E}+07$ & $1.08 \mathrm{E}+07$ & $1.52 \mathrm{E}+07$ & $1.14 \mathrm{E}+07$ & $1.30 \mathrm{E}+07$ & $3.69 \mathrm{E}+06$ & $9.90 \mathrm{E}+06$ \\
\hline Total Volume (gal) & $3.20 \mathrm{E}+06$ & $2.85 \mathrm{E}+06$ & $4.02 \mathrm{E}+06$ & $3.02 \mathrm{E}+06$ & $3.44 \mathrm{E}+06$ & $9.75 \mathrm{E}+05$ & $2.62 \mathrm{E}+06$ \\
\hline Total Mass (lb) & $3.46 \mathrm{E}+07$ & $3.08 \mathrm{E}+07$ & $4.35 \mathrm{E}+07$ & $3.26 \mathrm{E}+07$ & $3.72 \mathrm{E}+07$ & $1.05 \mathrm{E}+07$ & $2.83 \mathrm{E}+07$ \\
\hline Solution Sp. G. & $1.30 \mathrm{E}+00$ & $1.30 \mathrm{E}+00$ & $1.30 \mathrm{E}+00$ & $1.30 \mathrm{E}+00$ & $1.30 \mathrm{E}+00$ & $1.30 \mathrm{E}+00$ & $1.30 \mathrm{E}+00$ \\
\hline
\end{tabular}


Table 18: Projected Feed Volumes After Dissolution and Dilution to 6.44 M Total Sodium Concentration: 10\% interstitial supernate remaining

\begin{tabular}{|c|c|c|c|c|c|c|c|c|}
\hline \multicolumn{9}{|c|}{$600 \mathrm{mg} / \mathrm{L}$ Sludge- $10 \%$ Interstitial Supernate } \\
\hline \multicolumn{9}{|c|}{ Volume of Saltcake Components After Dilution To $6.44 \mathrm{M} \mathrm{Na}$} \\
\hline & Tank 25 & Tank 27 & \begin{tabular}{|l|} 
Tank 28 \\
\end{tabular} & Tank 29 & Tank 31 & Tank 33 & Tank 34 & Tank 36 \\
\hline Salt Volume (gal) & $1.11 \mathrm{E}+06$ & $4.63 E+05$ & $1.03 \mathrm{E}+06$ & $1.00 \mathrm{E}+06$ & $1.01 \mathrm{E}+06$ & $2.27 E+05$ & $2.12 \mathrm{E}+05$ & $1.09 \mathrm{E}+06$ \\
\hline Salt Mass (Ib) & $1.34 \mathrm{E}+07$ & $5.60 \mathrm{E}+06$ & $1.25 \mathrm{E}+07$ & $1.21 \mathrm{E}+07$ & $1.23 \mathrm{E}+07$ & $2.75 \mathrm{E}+06$ & $2.56 \mathrm{E}+06$ & $1.32 \mathrm{E}+07$ \\
\hline Salt Volume (L) & $4.19 \mathrm{E}+06$ & $1.75 \mathrm{E}+06$ & $3.91 \mathrm{E}+06$ & $3.79 \mathrm{E}+06$ & $3.84 \mathrm{E}+06$ & $8.59 \mathrm{E}+05$ & $8.03 E+05$ & $4.14 \mathrm{E}+06$ \\
\hline Intst. Supr. Vol. (L) & $9.23 \mathrm{E}+04$ & $3.86 \mathrm{E}+04$ & $8.59 \mathrm{E}+04$ & $8.33 \mathrm{E}+04$ & $8.44 \mathrm{E}+04$ & $1.89 \mathrm{E}+04$ & $1.77 \mathrm{E}+04$ & $9.11 \mathrm{E}+04$ \\
\hline Dilution Volume (L) & $1.02 \mathrm{E}+07$ & $4.27 \mathrm{E}+06$ & $9.51 \mathrm{E}+06$ & $1.03 \mathrm{E}+07$ & $1.06 \mathrm{E}+07$ & $2.06 \mathrm{E}+06$ & $1.95 \mathrm{E}+06$ & $1.14 \mathrm{E}+07$ \\
\hline Dilution Mass (Ib) & $2.25 \mathrm{E}+07$ & $9.41 \mathrm{E}+06$ & $2.09 \mathrm{E}+07$ & $2.27 \mathrm{E}+07$ & $2.33 \mathrm{E}+07$ & $4.53 E+06$ & $4.29 \mathrm{E}+06$ & $2.51 \mathrm{E}+07$ \\
\hline Total Volume (L) & $1.26 \mathrm{E}+07$ & $5.26 \mathrm{E}+06$ & $1.17 \mathrm{E}+07$ & $1.22 \mathrm{E}+07$ & $1.25 \mathrm{E}+07$ & $2.55 E+06$ & $2.40 E+06$ & $1.34 \mathrm{E}+07$ \\
\hline Total Volume (gal) & $3.32 \mathrm{E}+06$ & $1.39 \mathrm{E}+06$ & $3.09 E+06$ & $3.22 \mathrm{E}+06$ & $3.29 \mathrm{E}+06$ & $6.73 E+05$ & $6.34 \mathrm{E}+05$ & $3.55 \mathrm{E}+06$ \\
\hline Total Mass (Ib) & $3.59 \mathrm{E}+07$ & $1.50 \mathrm{E}+07$ & $3.34 \mathrm{E}+07$ & $3.48 \mathrm{E}+07$ & $3.56 \mathrm{E}+07$ & $7.27 \mathrm{E}+06$ & $6.85 E+06$ & $3.84 \mathrm{E}+07$ \\
\hline Solution Sp. G. & $1.30 \mathrm{E}+00$ & $1.30 \mathrm{E}+00$ & $1.30 \mathrm{E}+00$ & $1.30 \mathrm{E}+00$ & $1.30 \mathrm{E}+00$ & $1.30 \mathrm{E}+00$ & $1.30 \mathrm{E}+00$ & $1.30 \mathrm{E}+00$ \\
\hline
\end{tabular}

\begin{tabular}{|l|c|c|c|c|c|c|c|}
\hline \multicolumn{7}{|c|}{ Volume of Saltcake Components After Dilution To 6.44 M Na } \\
\hline & Tank 37 & Tank 38 & Tank 41 & Tank 44 & Tank 45 & Tank 46 & Tank 47 \\
\hline Salt Volume (gal) & $9.73 \mathrm{E}+05$ & $8.86 \mathrm{E}+05$ & $1.23 \mathrm{E}+06$ & $9.89 \mathrm{E}+05$ & $1.13 \mathrm{E}+06$ & $3.19 \mathrm{E}+05$ & $8.68 \mathrm{E}+05$ \\
\hline Salt Mass (Ib) & $1.18 \mathrm{E}+07$ & $1.07 \mathrm{E}+07$ & $1.49 \mathrm{E}+07$ & $1.20 \mathrm{E}+07$ & $1.37 \mathrm{E}+07$ & $3.86 \mathrm{E}+06$ & $1.05 \mathrm{E}+07$ \\
\hline Salt Volume (L) & $3.68 \mathrm{E}+06$ & $3.35 \mathrm{E}+06$ & $4.66 \mathrm{E}+06$ & $3.74 \mathrm{E}+06$ & $4.28 \mathrm{E}+06$ & $1.21 \mathrm{E}+06$ & $3.29 \mathrm{E}+06$ \\
\hline Intst. Supr. Vol. (L) & $8.10 \mathrm{E}+04$ & $7.38 \mathrm{E}+04$ & $1.03 \mathrm{E}+05$ & $8.24 \mathrm{E}+04$ & $9.41 \mathrm{E}+04$ & $2.66 \mathrm{E}+04$ & $7.23 \mathrm{E}+04$ \\
\hline Dilution Volume (L) & $1.01 \mathrm{E}+07$ & $9.07 \mathrm{E}+06$ & $1.28 \mathrm{E}+07$ & $9.14 \mathrm{E}+06$ & $1.04 \mathrm{E}+07$ & $2.95 \mathrm{E}+06$ & $7.94 \mathrm{E}+06$ \\
\hline Dilution Mass (Ib) & $2.24 \mathrm{E}+07$ & $2.00 \mathrm{E}+07$ & $2.82 \mathrm{E}+07$ & $2.01 \mathrm{E}+07$ & $2.30 \mathrm{E}+07$ & $6.50 \mathrm{E}+06$ & $1.75 \mathrm{E}+07$ \\
\hline Total Volume (L) & $1.20 \mathrm{E}+07$ & $1.08 \mathrm{E}+07$ & $1.51 \mathrm{E}+07$ & $1.12 \mathrm{E}+07$ & $1.28 \mathrm{E}+07$ & $3.63 \mathrm{E}+06$ & $9.80 \mathrm{E}+06$ \\
\hline Total Volume (gal) & $3.16 \mathrm{E}+06$ & $2.84 \mathrm{E}+06$ & $3.98 \mathrm{E}+06$ & $2.97 \mathrm{E}+06$ & $3.39 \mathrm{E}+06$ & $9.59 \mathrm{E}+05$ & $2.59 \mathrm{E}+06$ \\
\hline Total Mass (Ib) & $3.41 \mathrm{E}+07$ & $3.07 \mathrm{E}+07$ & $4.30 \mathrm{E}+07$ & $3.21 \mathrm{E}+07$ & $3.66 \mathrm{E}+07$ & $1.04 \mathrm{E}+07$ & $2.80 \mathrm{E}+07$ \\
\hline Solution Sp. G. & $1.30 \mathrm{E}+00$ & $1.30 \mathrm{E}+00$ & $1.30 \mathrm{E}+00$ & $1.30 \mathrm{E}+00$ & $1.30 \mathrm{E}+00$ & $1.30 \mathrm{E}+00$ & $1.30 \mathrm{E}+00$ \\
\hline
\end{tabular}




\section{Real Waste Salt Cake Samples}

Since 1994, several salt cake samples were extracted from Tanks 37, 38, and 41. ${ }^{5,6,7}$ These samples were obtained using a sampling cup that is driven into the surface of the salt cake. The material is held in the cup primarily by friction, but is aided by a retaining ring in some cases. These samples are nominally about $50 \mathrm{ml}$ each. Though not necessarily representative of the entire tank contents, these samples offer significant insight to the composition of the salt cake. The results from these samples are presented as if the material is dissolved and diluted with water to 6.44 $\mathrm{M}$ total sodium concentration. The composition predicted by WCS is presented in the adjacent column for comparison.

The WCS predicted composition assumes $70 \%$ drainage of interstitial liquid. Additional projections are provided if better drainage can be achieved. However, the samples from all three tanks were not drained to a specified supernate level. The sample technique compresses the salt cake, which forces out interstitial liquid. As the sample is removed from the tank, the remaining interstitial liquid is allowed to drain from the sample before packaging and shipping to the laboratory. If possible, the void fraction is estimated for each sample along with an estimate of retained supernate.

The weight fraction of supernate can be calculated as follows:

$$
\begin{aligned}
\mathrm{w}_{\text {supr }}= & \mathrm{M}_{\text {supr }} / \mathrm{M}_{\text {bulk }}=\mathrm{M}_{\mathrm{H} 2 \mathrm{O}} /\left(\mathrm{w}_{\mathrm{H} 2 \mathrm{O}-\text { sup }} * \mathrm{M}_{\text {bulk }}\right) \\
= & \mathrm{w}_{\mathrm{H} 2 \mathrm{O}-\text { bulk }} / \mathrm{w}_{\mathrm{H} 2 \mathrm{O} \text {-sup }}
\end{aligned}
$$

where

$$
\begin{aligned}
& \mathrm{M}_{\text {supr }}=\text { mass of supernate in sample } \\
& \mathrm{M}_{\text {bulk }}=\text { mass of sample } \\
& \mathrm{M}_{\mathrm{H} 20}=\text { mass of water in sample } \\
& \mathrm{v}_{\text {supr }}=\text { the volume fraction of the supernate in the sample } \\
& \mathrm{w}_{\mathrm{H} 2 \mathrm{O}-\text { bulk }}=\text { the weight fraction of the water in the sample } \\
& \mathrm{w}_{\mathrm{H} 2 \mathrm{O}-\text { sup }}=\text { the weight fraction of the water in the supernate, and } \\
& \mathrm{w}_{\text {supr }}=\text { the weight fraction of supernate in the sample. }
\end{aligned}
$$

The volume fraction of supernate in the sample can be calculated as follows:

$$
\begin{aligned}
\mathrm{v}_{\text {supr }}= & \mathrm{V}_{\text {supr }} / \mathrm{V}_{\text {bulk }}=\left(\mathrm{M}_{\text {supr }} / \rho_{\text {supr }}\right) /\left(\mathrm{M}_{\text {bulk }} / \rho_{\text {bulk }}\right) \\
& =\left(\mathrm{M}_{\text {supr }} / \mathrm{M}_{\text {bulk }}\right) *\left(\rho_{\text {bulk }} / \rho_{\text {supr }}\right) \\
& =\mathrm{w}_{\text {supr }} *\left(\rho_{\text {bulk }} / \rho_{\text {supr }}\right)
\end{aligned}
$$

where

$$
\rho_{\text {supr }} \text { and } \rho_{\text {bulk }}=\text { the densities of the supernate and bulk sample respectively. }
$$

Equation 10 is combined with equation 11 to yield

$$
\mathrm{v}_{\text {supr }}=\left(\mathrm{w}_{\mathrm{H} 2 \mathrm{O}-\mathrm{bulk}} / \mathrm{w}_{\mathrm{H} 2 \mathrm{O}-\text { sup }}\right) *\left(\rho_{\text {bulk }} / \rho_{\text {supr }}\right)
$$

By analogy to equation 12 ,

$$
\mathrm{v}_{\text {salt }}=\mathrm{w}_{\text {salt }} *\left(\rho_{\text {bulk }} / \rho_{\text {salt }}\right)
$$


WSRC-TR-2001-00559, Rev. 1

where

$$
\begin{aligned}
& \mathrm{v}_{\text {salt }}=\text { the volume fraction of the salt in the sample, and } \\
& \rho_{\text {salt }}=\text { the density of the salt. }
\end{aligned}
$$

Since $\mathrm{w}_{\text {supr }}+\mathrm{w}_{\text {salt }}=1$, neglecting the contribution of air, then

$$
\mathrm{v}_{\text {salt }}=\left(1-\mathrm{w}_{\text {supr }}\right) *\left(\rho_{\text {bulk }} / \rho_{\text {salt }}\right)
$$

Combining equation 10 with equation 14 results in

$$
\mathrm{v}_{\text {salt }}=\left(1-\mathrm{w}_{\text {H2O-bulk }} / \mathrm{w}_{\text {H2O-sup }}\right) *\left(\rho_{\text {bulk }} / \rho_{\text {salt }}\right)
$$

The volume fraction of air in the bulk sample was assumed to be the balance of the sample volume. The void fraction is the fraction of sample volume not occupied by salt or $\mathrm{v}_{\text {supr }}+\mathrm{v}_{\text {air }}$. Using the sample density and weight fraction water data in Table 19 and assuming the crystalline solids density is $2.2,{ }^{3}$ the calculated sample void fractions are $0.59,0.41$, and 0.42 for the Tank 37,38 , and 41 samples respectively.

Samples from all three tanks show much higher total insoluble solids than is predicted. These high insoluble solids content may be due to solids settling from the free supernate over a long period. Waste is not routinely transferred from or to these tanks. After long periods of stagnation, fine particles may settle as well as dust and corrosion debris. Aluminum precipitation as gibbsite and sodium aluminosilicates is known to occur slowly. All these mechanisms can contribute to the unusually high insoluble content of these samples. 
Table 19: Comparison of Real Salt Cake Sample Results with Predicted Compositions

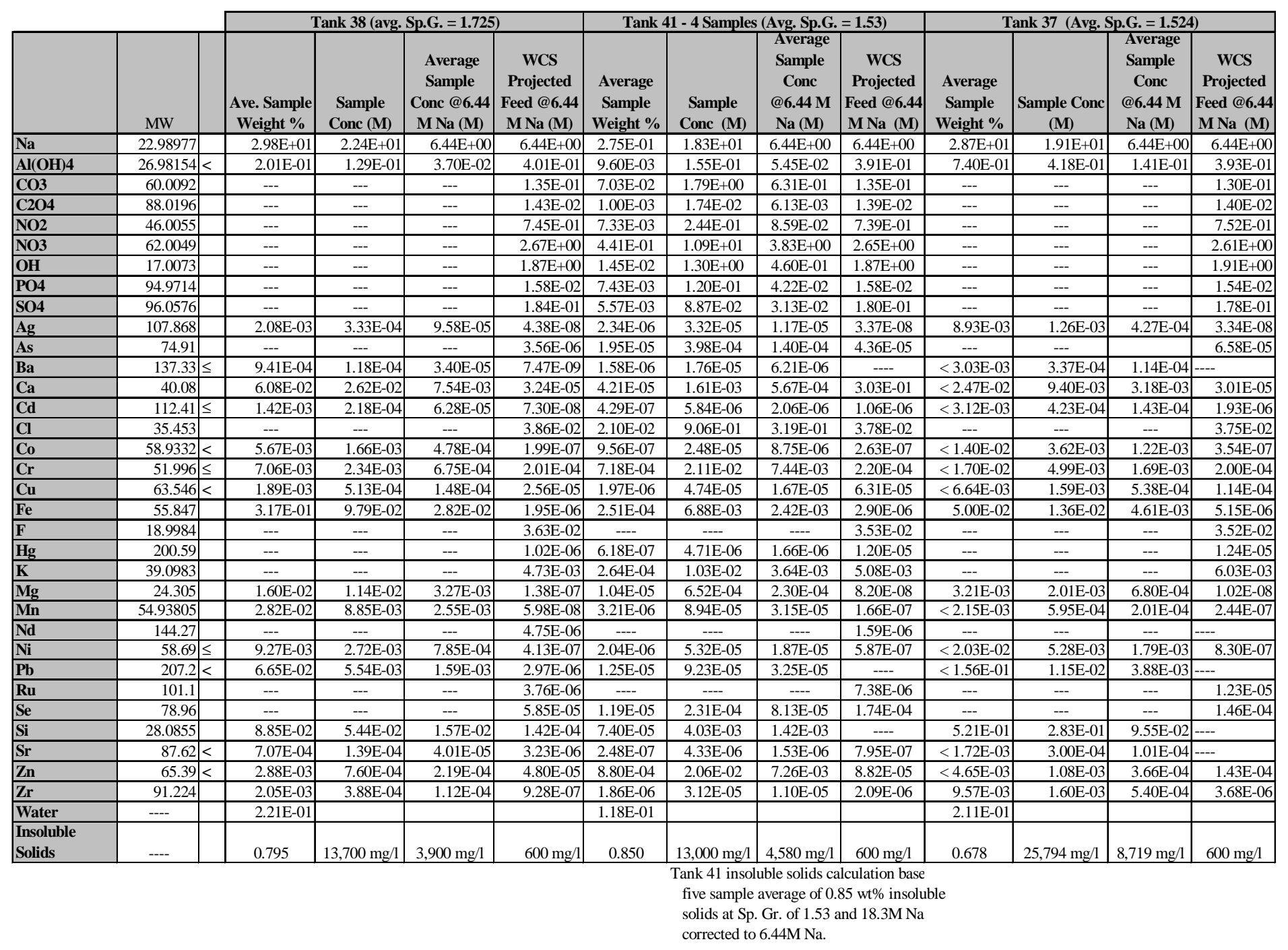


Table 19: Comparison of Real Salt Cake Sample Results with Predicted Compositions (Continued)

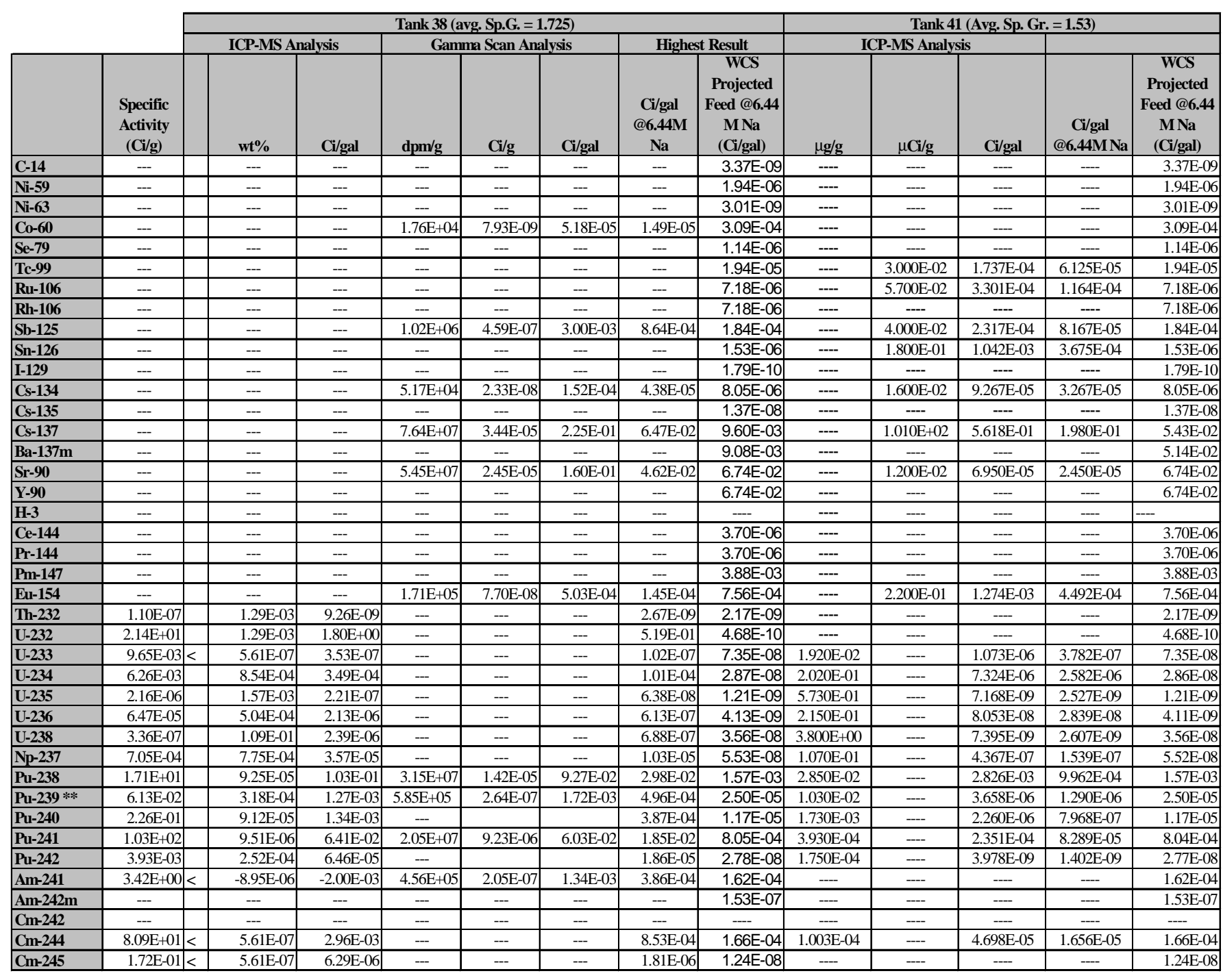


Table 19: Comparison of Real Salt Cake Sample Results with Predicted Compositions (continued)

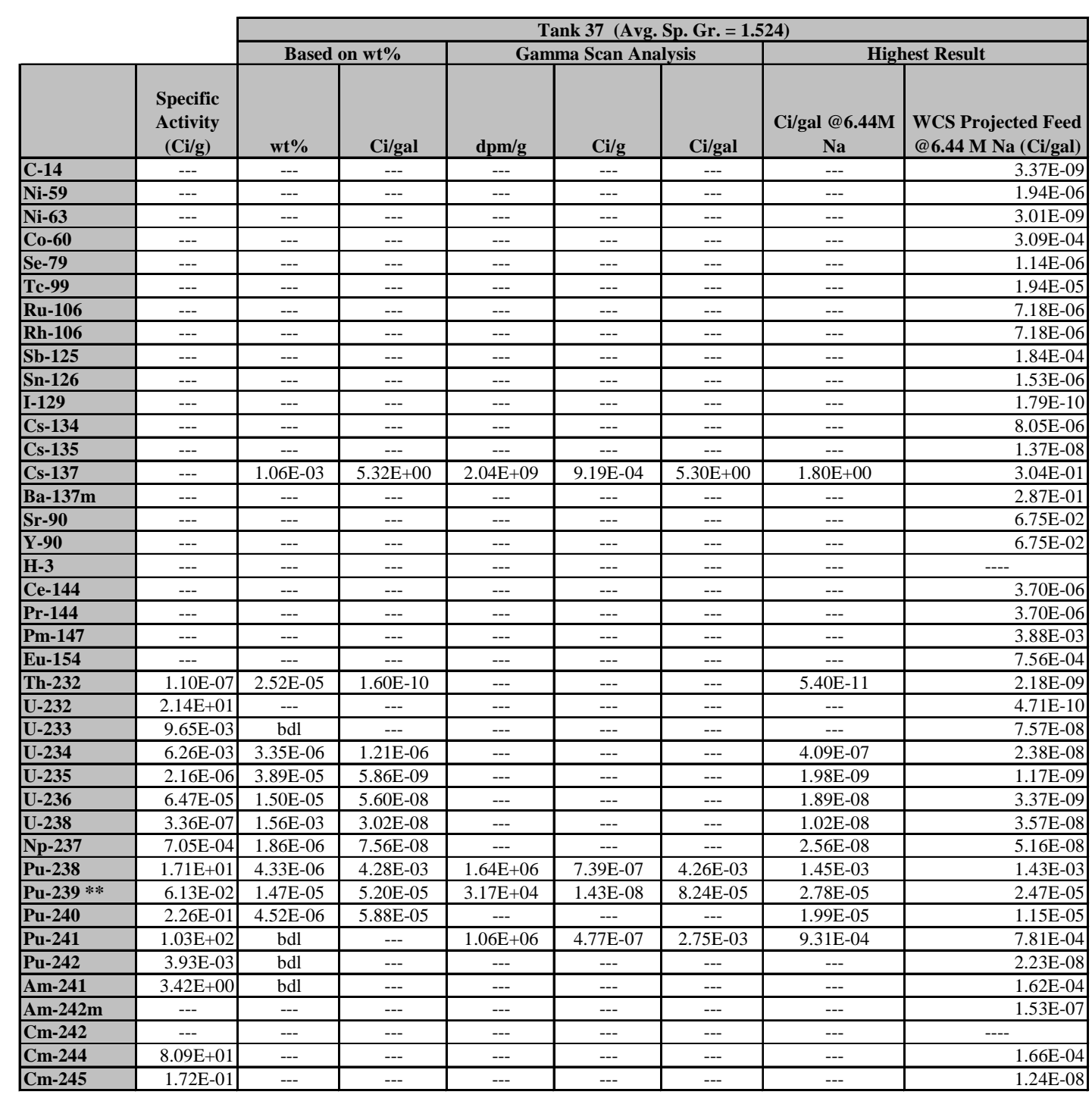




\section{References}

1 B. J. Wiersma, “An Investigation of Density Driven Salt Dissolution Techniques”, WSRC-TR-960160, August 1996.

2 J. N. Brooke, J. F. Peters, and K. Staheli, "Hydrological Methods can Separate Cesium from Nuclear Waste Salt Cake”, WSRC-TR-99-00358, October 1999.

3 J. R. Hester, "High Level Waste Characterization System (WCS)", WSRC-TR-96-0264, Rev. 0, December 1996.

4 F. A. Washburn, "SRS Soluble HLW Characterization”, HLW-SDT-2001-00310, Rev. 0, November, 2001.

5 L. N. Oji and R. F. Swingle, "Characterization of Tank 37H Salt Core Samples”, WSRC-TR-200100517, Rev. 0, November 12, 2001.

6 D. T. Hobbs and C. J. Coleman, "Final Report: Analysis of Tank 41H Saltcake Sample \#2 and Comparison to Sample \#1 (U)”, WSRC-TR-94-057, January 26, 1994.

$7 \quad$ R. F. Swingle, “Characterization of Tank 38H Variable Depth \& Core Samples”, WSRC-RP-200100574, Rev. 0, May 2001.

D. D. Walker, “Preparation of Simulated Waste Solutions”, WSRC-TR-99-00116, Rev. 0, April 1999. 
WSRC-TR-2001-00559, Rev. 1

\section{APPENDIX A}


WSRC-TR-2001-00559, Rev. 1

Tank 25

\begin{tabular}{|c|c|c|c|c|c|}
\hline \multirow{2}{*}{$\begin{array}{l}\text { Sample } \\
\text { Date }\end{array}$} & \multirow{2}{*}{$\begin{array}{l}\text { Samples DataBase } \\
\text { GROSS GAMMA }\end{array}$} & \multirow{2}{*}{$\begin{array}{l}\text { Samples DataBase } \\
\text { GROSS GAMMA (Ci/gal) }\end{array}$} & \multirow{2}{*}{$\begin{array}{l}\text { Samples Database } \\
\text { Cs-137 (d/m/ml) }\end{array}$} & \multirow{2}{*}{$\begin{array}{l}\text { Samples Database } \\
\text { Cs-137 (Ci/gal) }\end{array}$} & \multirow{2}{*}{$\begin{array}{c}\text { WCS } \\
\mathrm{Ci} / \mathrm{gal} \\
4.98\end{array}$} \\
\hline & & & & & \\
\hline 02/12/98 & $2.38 \mathrm{E}+09$ & $4.06 \mathrm{E}+00$ & & & \\
\hline 04/10/97 & & & $2.57 \mathrm{E}+09$ & $4.38 \mathrm{E}+00$ & \\
\hline 02/13/97 & $2.58 \mathrm{E}+09$ & $4.40 \mathrm{E}+00$ & $2.57 \mathrm{E}+09$ & $4.38 \mathrm{E}+00$ & Average (3) \\
\hline 01/13/97 & $2.73 E+09$ & $4.65 \mathrm{E}+00$ & & & 4.37 \\
\hline 12/01/95 & $2.68 \mathrm{E}+09$ & $4.57 \mathrm{E}+00$ & & & \\
\hline $11 / 30 / 95$ & & & & & \\
\hline 08/31/95 & $2.74 \mathrm{E}+09$ & $4.67 \mathrm{E}+00$ & & & Date Range \\
\hline 08/18/94 & $3.11 \mathrm{E}+09$ & $5.30 \mathrm{E}+00$ & & & of Average \\
\hline 08/05/93 & $2.76 \mathrm{E}+09$ & $4.71 \mathrm{E}+00$ & $2.76 \mathrm{E}+09$ & $4.71 \mathrm{E}+00$ & 1/13/97-2/12/98 \\
\hline 07/30/92 & $3.39 E+09$ & $5.78 \mathrm{E}+00$ & $2.50 \mathrm{E}+09$ & $4.26 \mathrm{E}+00$ & \\
\hline 07/30/92 & $3.39 E+09$ & $5.78 \mathrm{E}+00$ & $3.39 \mathrm{E}+09$ & $5.78 \mathrm{E}+00$ & \\
\hline 07/30/92 & & & $2.50 \mathrm{E}+09$ & $4.26 \mathrm{E}+00$ & \\
\hline 07/26/91 & $2.96 \mathrm{E}+09$ & $5.05 \mathrm{E}+00$ & & & \\
\hline 05/15/89 & $2.24 \mathrm{E}+09$ & $3.82 \mathrm{E}+00$ & & & \\
\hline $11 / 04 / 88$ & $1.78 \mathrm{E}+09$ & $3.04 \mathrm{E}+00$ & & & \\
\hline 04/21/88 & $3.74 \mathrm{E}+09$ & $6.38 \mathrm{E}+00$ & $5.52 \mathrm{E}+08$ & $9.41 \mathrm{E}-01$ & \\
\hline 10/07/87 & $2.48 \mathrm{E}+09$ & $4.23 \mathrm{E}+00$ & $4.23 \mathrm{E}+08$ & 7.21E-01 & \\
\hline 04/04/86 & & & & & \\
\hline 08/19/85 & & & $5.10 \mathrm{E}+08$ & $8.70 \mathrm{E}-01$ & \\
\hline 04/09/85 & & & $4.35 \mathrm{E}+08$ & 7.42E-01 & \\
\hline 03/19/85 & & & & & \\
\hline 02/09/84 & & & & & \\
\hline 03/18/83 & & & & & \\
\hline 06/30/82 & & & & & \\
\hline $11 / 10 / 81$ & & & & & \\
\hline $11 / 06 / 81$ & & & & & \\
\hline $10 / 16 / 81$ & & & & & \\
\hline 04/08/81 & & & & & \\
\hline $09 / 22 / 80$ & & & & & \\
\hline
\end{tabular}

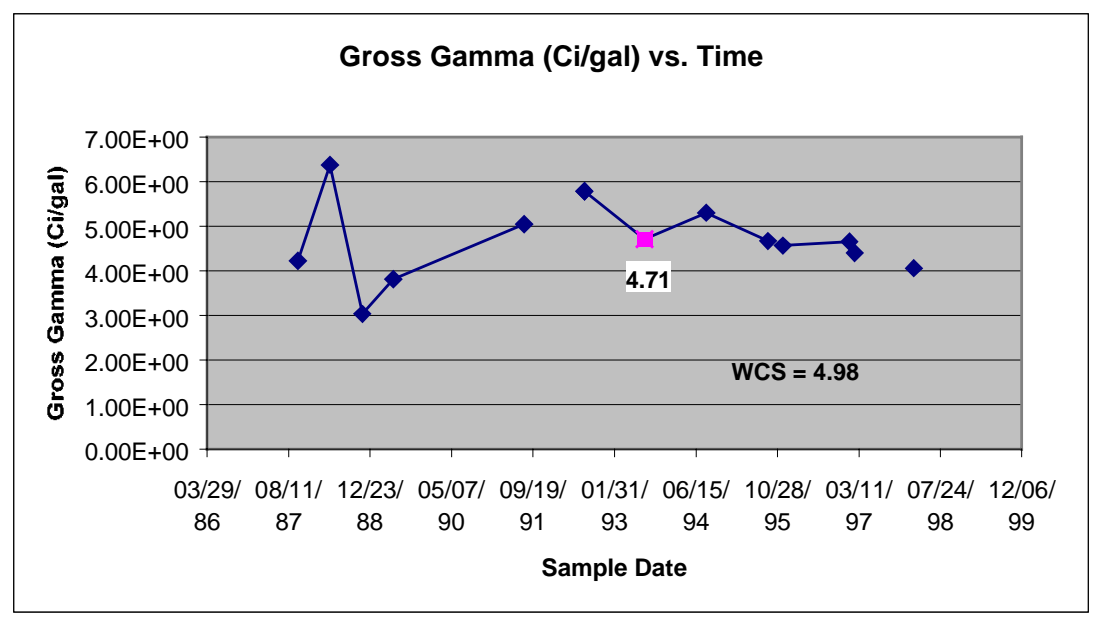


Tank 27

\begin{tabular}{|c|c|c|c|c|c|}
\hline $\begin{array}{l}\text { Sample } \\
\text { Date }\end{array}$ & $\begin{array}{l}\text { Samples DataBase } \\
\text { GROSS GAMMA }\end{array}$ & $\begin{array}{l}\text { Samples DataBase } \\
\text { GROSS GAMMA (Ci/gal) }\end{array}$ & $\begin{array}{l}\text { Samples Database } \\
\text { Cs-137 (d/m/ml) }\end{array}$ & $\begin{array}{l}\text { Samples Database } \\
\text { Cs-137 (Ci/gal) }\end{array}$ & $\begin{array}{c}\text { WCS } \\
\mathrm{Ci} / \mathrm{gal} \\
4.96\end{array}$ \\
\hline 06/24/99 & $2.26 \mathrm{E}+09$ & $3.85 \mathrm{E}+00$ & & & \\
\hline 07/08/97 & $1.37 \mathrm{E}+09$ & $2.34 \mathrm{E}+00$ & & & \\
\hline 07/09/96 & $2.55 \mathrm{E}+09$ & $4.35 \mathrm{E}+00$ & & & Average (3) \\
\hline 08/21/95 & $2.25 \mathrm{E}+09$ & $3.84 \mathrm{E}+00$ & & & 3.51 \\
\hline $08 / 18 / 94$ & $3.56 \mathrm{E}+09$ & $6.07 E+00$ & & & \\
\hline 08/05/93 & $2.75 \mathrm{E}+09$ & $4.69 \mathrm{E}+00$ & $2.75 \mathrm{E}+09$ & $4.69 \mathrm{E}+00$ & \\
\hline 07/21/92 & $3.16 \mathrm{E}+09$ & $5.39 \mathrm{E}+00$ & $3.16 \mathrm{E}+09$ & $5.39 \mathrm{E}+00$ & Date Range \\
\hline 07/21/92 & $3.16 \mathrm{E}+09$ & $5.39 \mathrm{E}+00$ & $3.16 \mathrm{E}+09$ & $5.39 \mathrm{E}+00$ & of Average \\
\hline 07/21/92 & & & $2.20 \mathrm{E}+09$ & $3.75 \mathrm{E}+00$ & 7/9/96-6/24/99 \\
\hline 08/02/91 & $2.96 \mathrm{E}+09$ & $5.05 \mathrm{E}+00$ & & & \\
\hline $05 / 24 / 90$ & $5.01 \mathrm{E}+09$ & $8.54 \mathrm{E}+00$ & & & \\
\hline 05/15/89 & $3.52 \mathrm{E}+09$ & $6.00 \mathrm{E}+00$ & & & \\
\hline $11 / 04 / 88$ & $2.00 \mathrm{E}+09$ & $3.41 \mathrm{E}+00$ & & & \\
\hline 05/18/88 & $4.67 \mathrm{E}+07$ & 7.96E-02 & $1.84 \mathrm{E}+06$ & $3.14 \mathrm{E}-03$ & \\
\hline $04 / 21 / 88$ & $4.67 \mathrm{E}+07$ & 7.96E-02 & & & \\
\hline $10 / 07 / 87$ & $2.29 \mathrm{E}+09$ & $3.90 \mathrm{E}+00$ & $3.80 \mathrm{E}+08$ & $6.48 \mathrm{E}-01$ & \\
\hline 04/03/87 & $2.60 \mathrm{E}+09$ & $4.43 \mathrm{E}+00$ & $4.48 \mathrm{E}+08$ & 7.64E-01 & \\
\hline $11 / 25 / 86$ & $3.11 \mathrm{E}+09$ & $5.30 \mathrm{E}+00$ & $4.11 \mathrm{E}+08$ & 7.01E-01 & \\
\hline \multicolumn{6}{|l|}{$10 / 20 / 84$} \\
\hline \multicolumn{6}{|l|}{ 02/09/84 } \\
\hline \multicolumn{6}{|l|}{ 08/23/83 } \\
\hline \multicolumn{6}{|l|}{$03 / 18 / 83$} \\
\hline \multicolumn{6}{|l|}{ 03/25/82 } \\
\hline \multicolumn{6}{|l|}{$10 / 16 / 81$} \\
\hline \multicolumn{6}{|l|}{ 08/19/80 } \\
\hline \multicolumn{6}{|l|}{$03 / 06 / 80$} \\
\hline \multicolumn{6}{|l|}{$02 / 01 / 80$} \\
\hline \multicolumn{6}{|l|}{$\begin{array}{l}01 / 25 / 80 \\
01 / 24 / 80\end{array}$} \\
\hline $01 / 24 / 80$ & & & & & \\
\hline
\end{tabular}

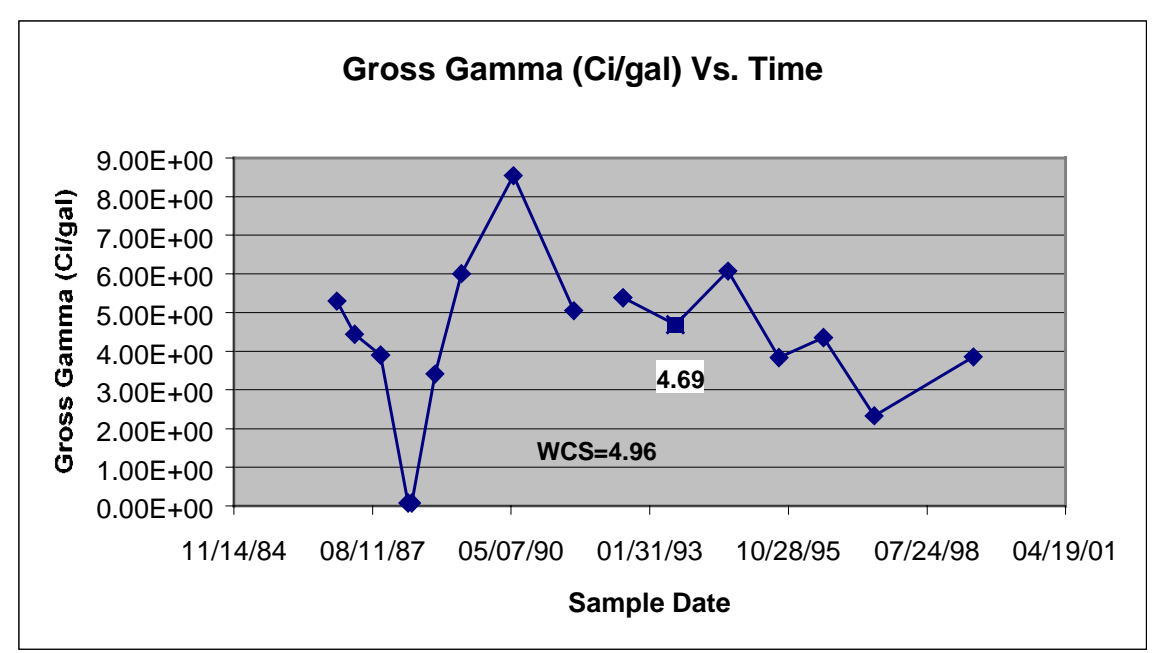


WSRC-TR-2001-00559, Rev. 1

Tank 28

\begin{tabular}{|c|c|c|c|c|c|}
\hline Sample & Samples DataBase & Samples DataBase & Samples Database & Samples Database & WCS \\
\hline Date & GROSS GAMMA & GROSS GAMMA (Ci/gal) & Cs-137 (d/m/ml) & Cs-137 (Ci/gal) & $\begin{array}{c}\mathrm{Ci} / \mathrm{gal} \\
4.12\end{array}$ \\
\hline 06/29/99 & $2.48 \mathrm{E}+09$ & $4.23 \mathrm{E}+00$ & & & \\
\hline 08/26/97 & $2.92 \mathrm{E}+09$ & $4.98 \mathrm{E}+00$ & & & \\
\hline 08/26/96 & $2.87 \mathrm{E}+09$ & $4.89 \mathrm{E}+00$ & & & Average (3) \\
\hline 08/31/95 & $3.30 \mathrm{E}+09$ & $5.63 \mathrm{E}+00$ & & & 4.70 \\
\hline 07/08/94 & $2.78 \mathrm{E}+09$ & $4.74 \mathrm{E}+00$ & & & \\
\hline 07/23/93 & $2.29 \mathrm{E}+09$ & $3.90 \mathrm{E}+00$ & $2.29 \mathrm{E}+09$ & $3.90 \mathrm{E}+00$ & \\
\hline 07/21/92 & $3.57 \mathrm{E}+09$ & $6.09 \mathrm{E}+00$ & 3.57E+09 & $6.09 \mathrm{E}+00$ & Date Range \\
\hline 07/21/92 & $3.57 \mathrm{E}+09$ & $6.09 \mathrm{E}+00$ & 3.57E+09 & $6.09 \mathrm{E}+00$ & of Average \\
\hline 07/21/92 & & & $2.60 \mathrm{E}+09$ & $4.43 \mathrm{E}+00$ & 8/26/96-6/29/99 \\
\hline 07/26/91 & $7.21 \mathrm{E}+08$ & $1.23 \mathrm{E}+00$ & & & \\
\hline 05/24/90 & $4.98 \mathrm{E}+09$ & $8.49 \mathrm{E}+00$ & & & \\
\hline 05/15/89 & $1.38 \mathrm{E}+09$ & $2.35 \mathrm{E}+00$ & & & \\
\hline $11 / 04 / 88$ & $3.13 E+09$ & $5.34 \mathrm{E}+00$ & & & \\
\hline $04 / 21 / 88$ & $3.46 \mathrm{E}+09$ & $5.90 \mathrm{E}+00$ & $4.52 \mathrm{E}+08$ & 7.71E-01 & \\
\hline $10 / 07 / 87$ & $2.54 \mathrm{E}+09$ & $4.33 \mathrm{E}+00$ & $4.12 \mathrm{E}+08$ & $7.02 \mathrm{E}-01$ & \\
\hline $11 / 26 / 86$ & $1.85 E+09$ & $3.15 \mathrm{E}+00$ & $2.39 \mathrm{E}+08$ & $4.08 \mathrm{E}-01$ & \\
\hline 04/04/86 & $4.76 \mathrm{E}+09$ & $8.12 \mathrm{E}+00$ & & & \\
\hline 08/19/85 & $3.40 \mathrm{E}+09$ & $5.80 \mathrm{E}+00$ & $4.61 \mathrm{E}+08$ & 7.86E-01 & \\
\hline 04/09/85 & & & $6.20 \mathrm{E}+08$ & $1.06 \mathrm{E}+00$ & \\
\hline \multicolumn{6}{|l|}{ 03/19/85 } \\
\hline \multicolumn{6}{|l|}{$12 / 01 / 83$} \\
\hline \multicolumn{6}{|l|}{ 03/18/83 } \\
\hline \multicolumn{6}{|l|}{ 04/22/82 } \\
\hline 02/03/81 & & & $2.38 \mathrm{E}+09$ & $4.06 \mathrm{E}+00$ & \\
\hline \multicolumn{6}{|l|}{$10 / 16 / 81$} \\
\hline \multicolumn{6}{|l|}{ 04/08/81 } \\
\hline \multicolumn{6}{|l|}{$11 / 10 / 80$} \\
\hline \multicolumn{6}{|l|}{ 09/08/80 } \\
\hline \multicolumn{6}{|l|}{$03 / 13 / 80$} \\
\hline $02 / 01 / 80$ & & & & & \\
\hline
\end{tabular}

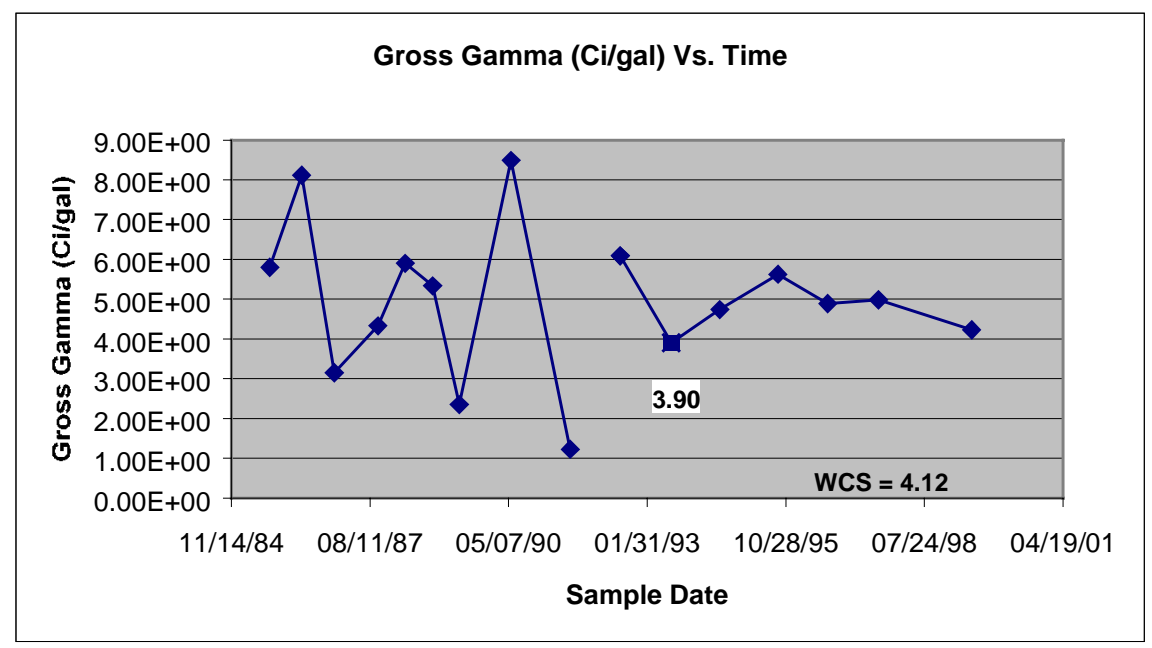


WSRC-TR-2001-00559, Rev. 1

Tank 29

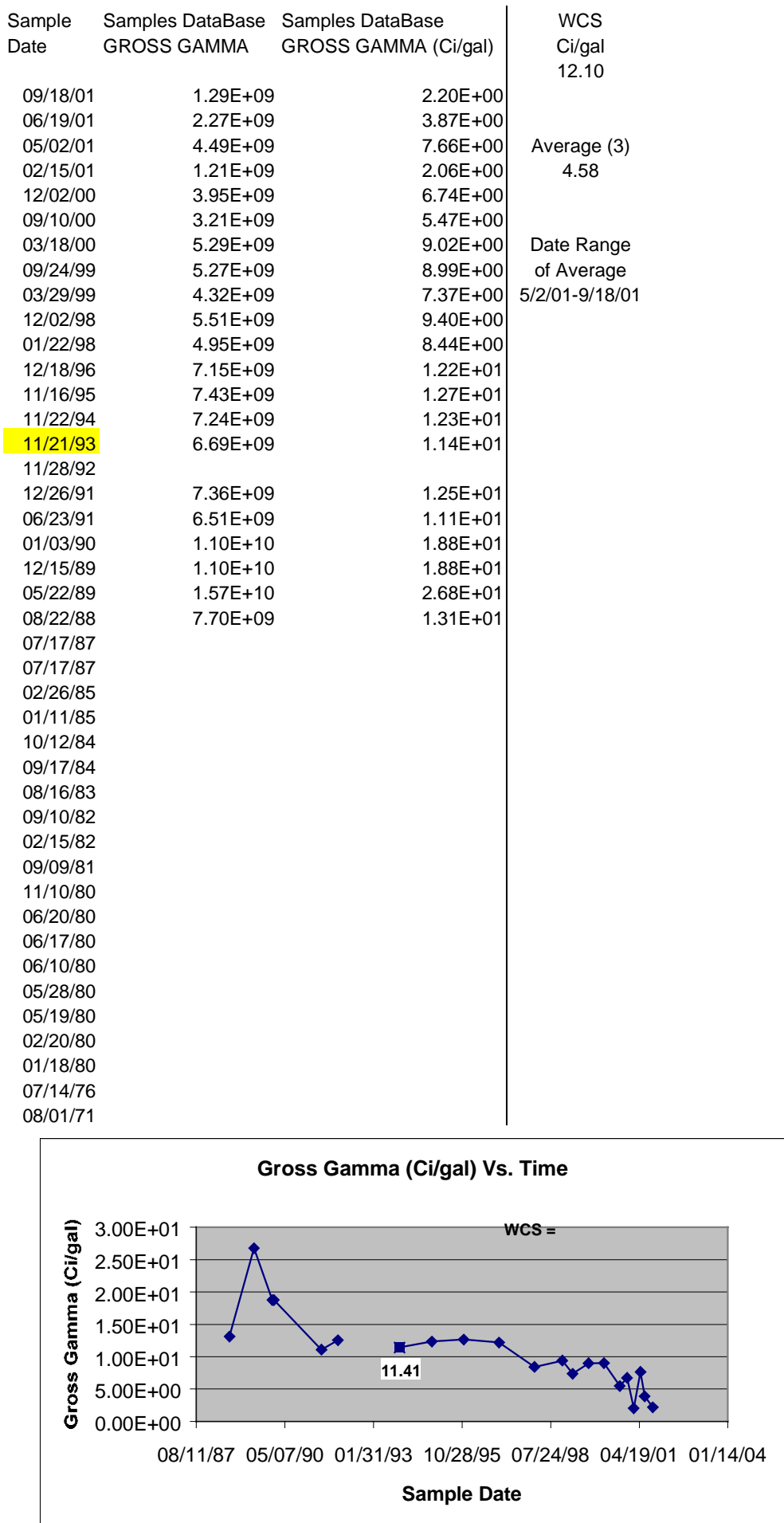


WSRC-TR-2001-00559, Rev. 1

Tank 31

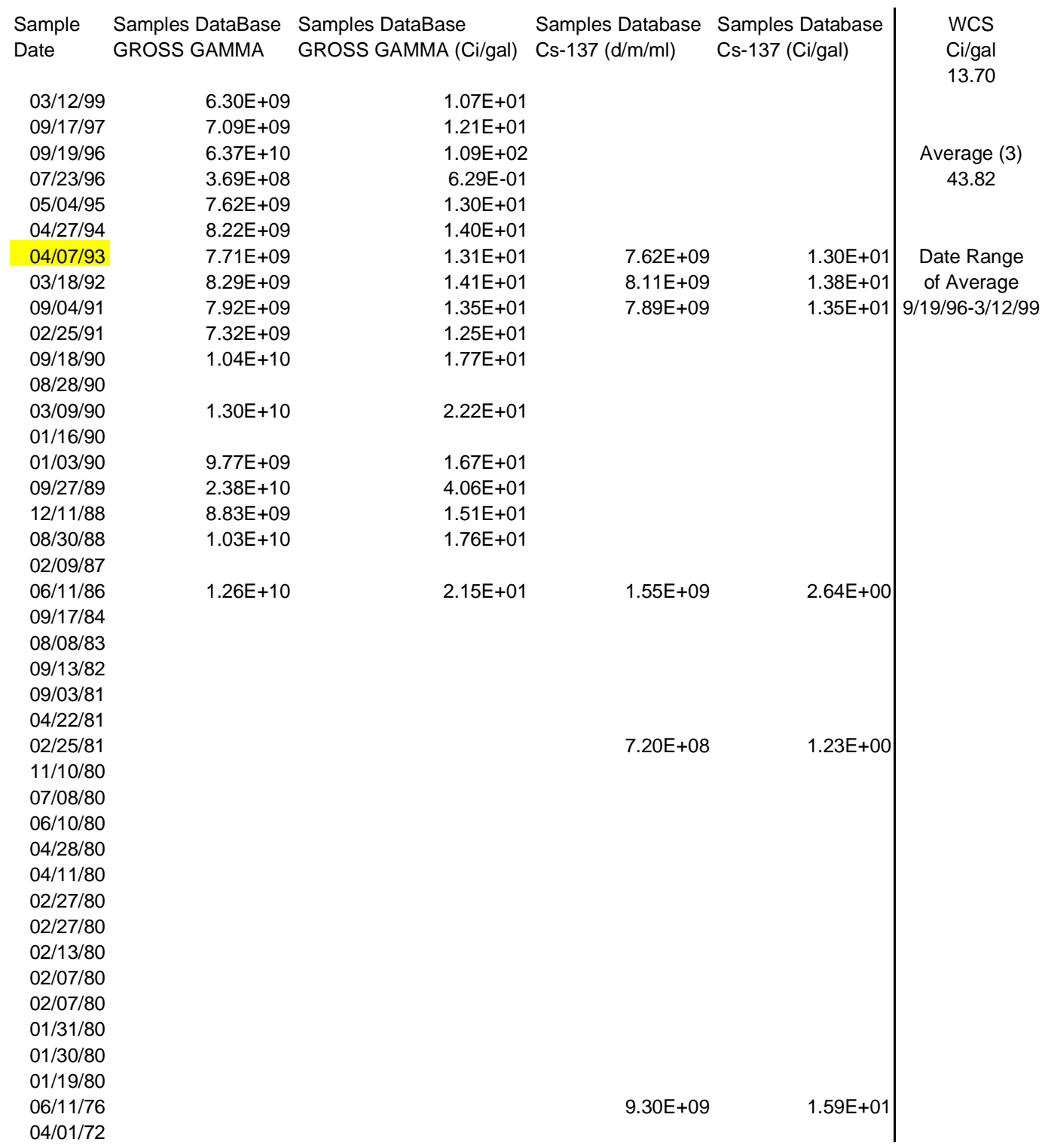

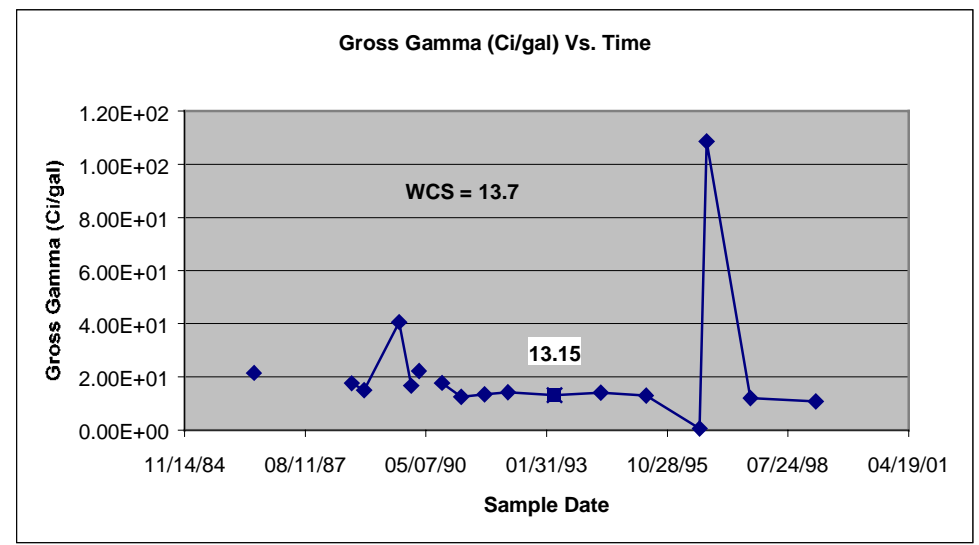


WSRC-TR-2001-00559, Rev. 1

Tank 33

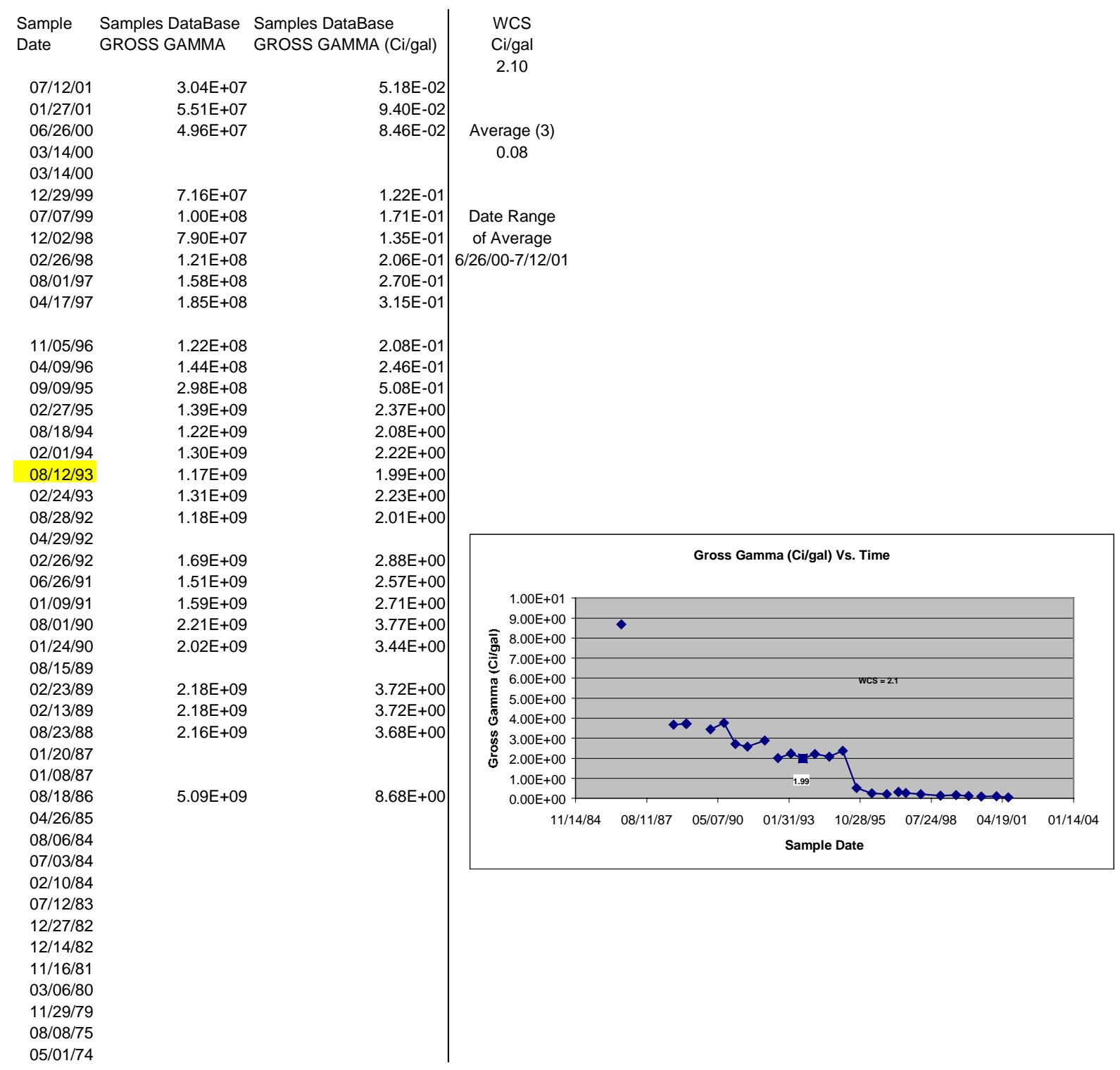


WSRC-TR-2001-00559, Rev. 1

Tank 34

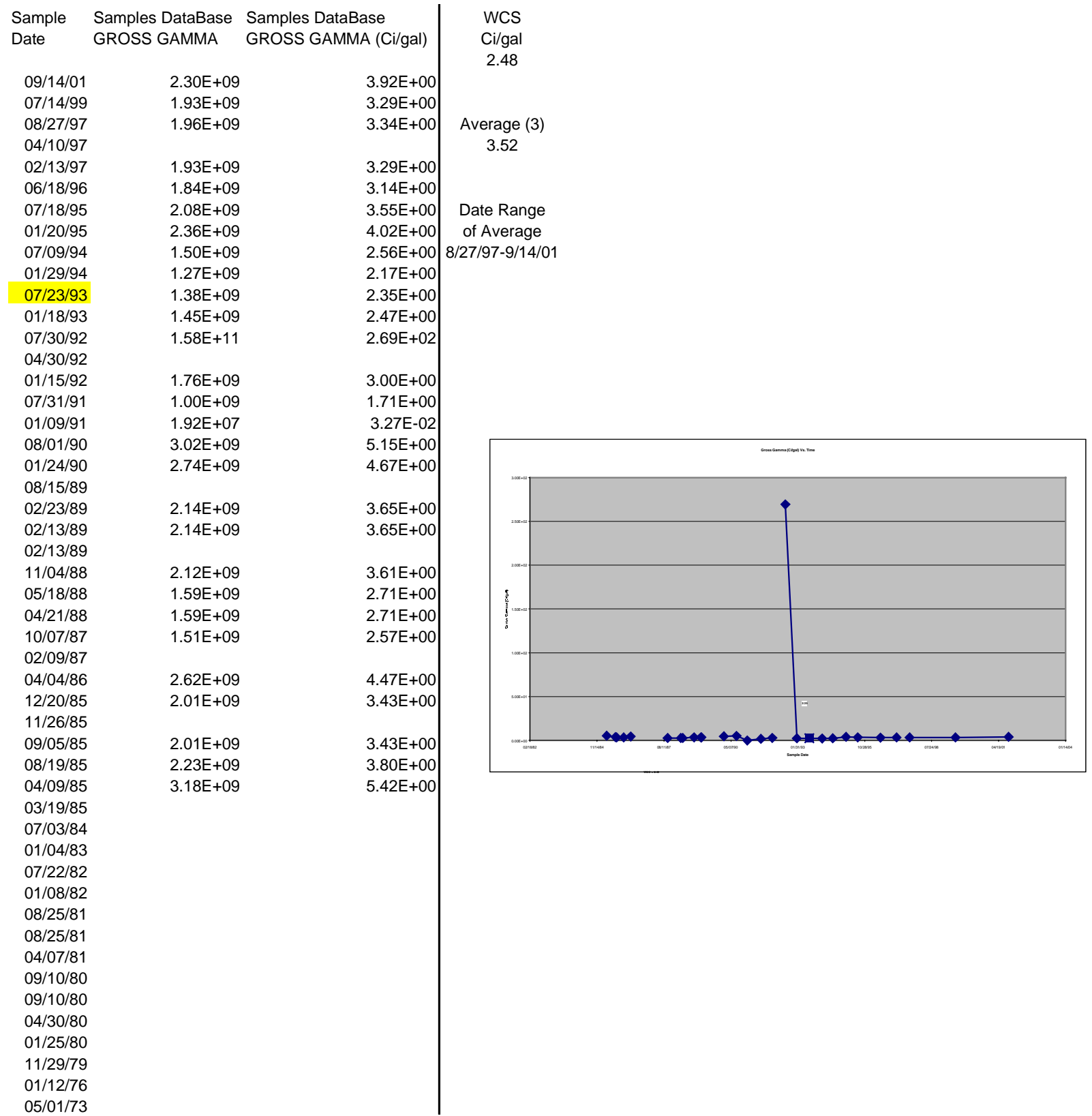


Tank 36

\begin{tabular}{|c|c|c|c|c|c|}
\hline Sample & Samples DataBase & Samples DataBase & Samples Database & Samples Database & WCS \\
\hline Date & GROSS GAMMA & GROSS GAMMA (Ci/gal) & Cs-137 (d/m/ml) & Cs-137 (Ci/gal) & $\begin{array}{c}\mathrm{Ci} / \mathrm{gal} \\
4.38\end{array}$ \\
\hline $12 / 09 / 98$ & $1.16 \mathrm{E}+10$ & $1.98 \mathrm{E}+01$ & & & \\
\hline 09/17/97 & $1.27 \mathrm{E}+10$ & $2.17 \mathrm{E}+01$ & & & \\
\hline 09/01/97 & $1.22 \mathrm{E}+10$ & $2.08 \mathrm{E}+01$ & & & Average (3) \\
\hline 08/22/96 & $1.23 \mathrm{E}+10$ & $2.10 \mathrm{E}+01$ & & & 20.75 \\
\hline 05/04/95 & $1.38 \mathrm{E}+10$ & $2.35 \mathrm{E}+01$ & & & \\
\hline $04 / 04 / 94$ & $1.37 \mathrm{E}+10$ & $2.34 \mathrm{E}+01$ & $1.37 \mathrm{E}+10$ & $2.34 \mathrm{E}+01$ & \\
\hline 03/28/93 & $2.43 \mathrm{E}+09$ & $4.14 \mathrm{E}+00$ & $2.43 \mathrm{E}+09$ & $4.14 \mathrm{E}+00$ & Date Range \\
\hline 03/28/93 & $9.06 \mathrm{E}+08$ & $1.54 \mathrm{E}+00$ & $8.89 \mathrm{E}+08$ & $1.52 \mathrm{E}+00$ & of Average \\
\hline 03/02/92 & $1.92 \mathrm{E}+10$ & $3.27 \mathrm{E}+01$ & & & 9/1/97-12/9/98 \\
\hline 03/09/90 & $2.56 \mathrm{E}+10$ & 4.37E+01 & & & \\
\hline 01/03/90 & $2.35 \mathrm{E}+10$ & $4.01 \mathrm{E}+01$ & & & \\
\hline 09/29/89 & $4.28 \mathrm{E}+10$ & $7.30 \mathrm{E}+01$ & & & \\
\hline 03/20/89 & $1.58 \mathrm{E}+10$ & $2.69 \mathrm{E}+01$ & & & \\
\hline $03 / 21 / 88$ & $9.64 \mathrm{E}+09$ & $1.64 \mathrm{E}+01$ & $1.41 \mathrm{E}+09$ & $2.40 \mathrm{E}+00$ & \\
\hline 09/28/87 & $1.08 \mathrm{E}+10$ & $1.84 \mathrm{E}+01$ & $1.64 \mathrm{E}+09$ & $2.80 \mathrm{E}+00$ & \\
\hline $04 / 02 / 87$ & $1.52 \mathrm{E}+10$ & $2.59 \mathrm{E}+01$ & $2.02 \mathrm{E}+09$ & $3.44 \mathrm{E}+00$ & \\
\hline 03/23/87 & & & & & \\
\hline $04 / 21 / 86$ & $4.90 \mathrm{E}+10$ & $8.35 \mathrm{E}+01$ & $1.47 \mathrm{E}+09$ & $2.51 \mathrm{E}+00$ & \\
\hline 08/05/83 & & & & & \\
\hline 07/17/82 & & & & & \\
\hline 07/17/82 & & & & & \\
\hline 09/03/81 & & & & & \\
\hline 09/03/81 & & & & & \\
\hline $02 / 14 / 81$ & & & & & \\
\hline 02/03/81 & & & & & \\
\hline $11 / 19 / 80$ & & & & & \\
\hline 02/25/80 & & & & & \\
\hline $05 / 17$ & & & & & \\
\hline
\end{tabular}

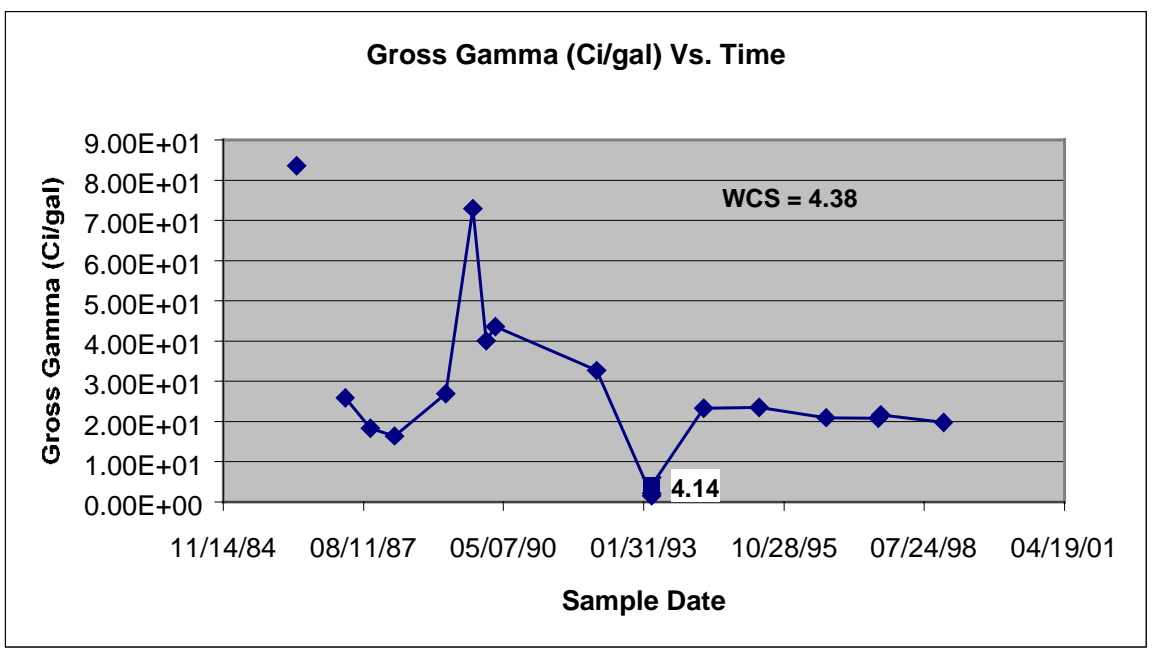


WSRC-TR-2001-00559, Rev. 1

Tank 37

\begin{tabular}{|c|c|c|c|c|c|}
\hline Sample & Samples DataBase & Samples DataBase & Samples Database & Samples Database & WCS \\
\hline Date & GROSS GAMMA & GROSS GAMMA (Ci/gal) & Cs-137 (d/m/ml) & Cs-137 (Ci/gal) & $\begin{array}{c}\mathrm{Ci} / \mathrm{gal} \\
1.91\end{array}$ \\
\hline 09/14/01 & 7.37E+09 & $1.26 \mathrm{E}+01$ & & & \\
\hline 03/12/99 & $8.42 \mathrm{E}+09$ & $1.44 \mathrm{E}+01$ & & & \\
\hline 06/29/97 & $8.53 E+09$ & $1.45 \mathrm{E}+01$ & & & Average (3) \\
\hline 04/24/96 & $6.96 \mathrm{E}+09$ & $1.19 \mathrm{E}+01$ & & & 13.82 \\
\hline 03/27/95 & 7.87E+09 & $1.34 \mathrm{E}+01$ & & & \\
\hline 03/24/94 & $5.55 \mathrm{E}+09$ & $9.46 \mathrm{E}+00$ & $5.55 \mathrm{E}+09$ & $9.46 \mathrm{E}+00$ & \\
\hline 03/29/93 & $1.06 \mathrm{E}+09$ & $1.81 \mathrm{E}+00$ & $1.06 \mathrm{E}+09$ & $1.81 \mathrm{E}+00$ & Date Range \\
\hline 03/29/93 & $1.63 \mathrm{E}+09$ & $2.78 \mathrm{E}+00$ & $1.62 \mathrm{E}+09$ & $2.76 \mathrm{E}+00$ & of Average \\
\hline 03/18/92 & $4.50 \mathrm{E}+09$ & 7.67E+00 & 4.32E+09 & 7.37E+00 & 6/29/97-9/14/01 \\
\hline 09/04/91 & & & $3.35 \mathrm{E}+09$ & $5.71 \mathrm{E}+00$ & \\
\hline 02/25/91 & $1.63 \mathrm{E}+10$ & $2.78 \mathrm{E}+01$ & & & \\
\hline $01 / 23 / 91$ & $1.38 \mathrm{E}+10$ & $2.35 \mathrm{E}+01$ & & & \\
\hline 08/28/90 & $1.97 \mathrm{E}+10$ & 3.36E+01 & & & \\
\hline 03/09/90 & $2.64 \mathrm{E}+10$ & $4.50 \mathrm{E}+01$ & & & \\
\hline 01/04/90 & $2.14 \mathrm{E}+10$ & $3.65 \mathrm{E}+01$ & & & \\
\hline 09/29/89 & $4.31 \mathrm{E}+10$ & $7.35 \mathrm{E}+01$ & & & \\
\hline 03/20/89 & $1.49 \mathrm{E}+10$ & $2.54 \mathrm{E}+01$ & & & \\
\hline 03/21/88 & $1.45 \mathrm{E}+10$ & $2.47 \mathrm{E}+01$ & $1.88 \mathrm{E}+09$ & $3.21 \mathrm{E}+00$ & \\
\hline $09 / 28 / 87$ & $1.02 \mathrm{E}+10$ & $1.74 \mathrm{E}+01$ & $1.49 \mathrm{E}+09$ & $2.54 \mathrm{E}+00$ & \\
\hline 04/01/87 & 1.37E+10 & $2.34 \mathrm{E}+01$ & $1.92 \mathrm{E}+09$ & $3.27 \mathrm{E}+00$ & \\
\hline $03 / 23 / 87$ & & & & & \\
\hline $06 / 11 / 86$ & $2.01 \mathrm{E}+10$ & $3.43 \mathrm{E}+01$ & $2.51 \mathrm{E}+09$ & $4.28 \mathrm{E}+00$ & \\
\hline $08 / 16 / 83$ & & & & & \\
\hline 07/17/82 & & & & & \\
\hline 09/09/81 & & & & & \\
\hline 02/27/81 & & & & & \\
\hline 02/14/81 & & & & & \\
\hline 02/03/81 & & & & & \\
\hline $12 / 05 / 80$ & & & & & \\
\hline $11 / 19 / 80$ & & & & & \\
\hline 06/10/80 & & & & & \\
\hline $02 / 25 / 80$ & & & & & \\
\hline $08 / 20 / 79$ & & & & & \\
\hline 05/18/79 & & & & & \\
\hline
\end{tabular}

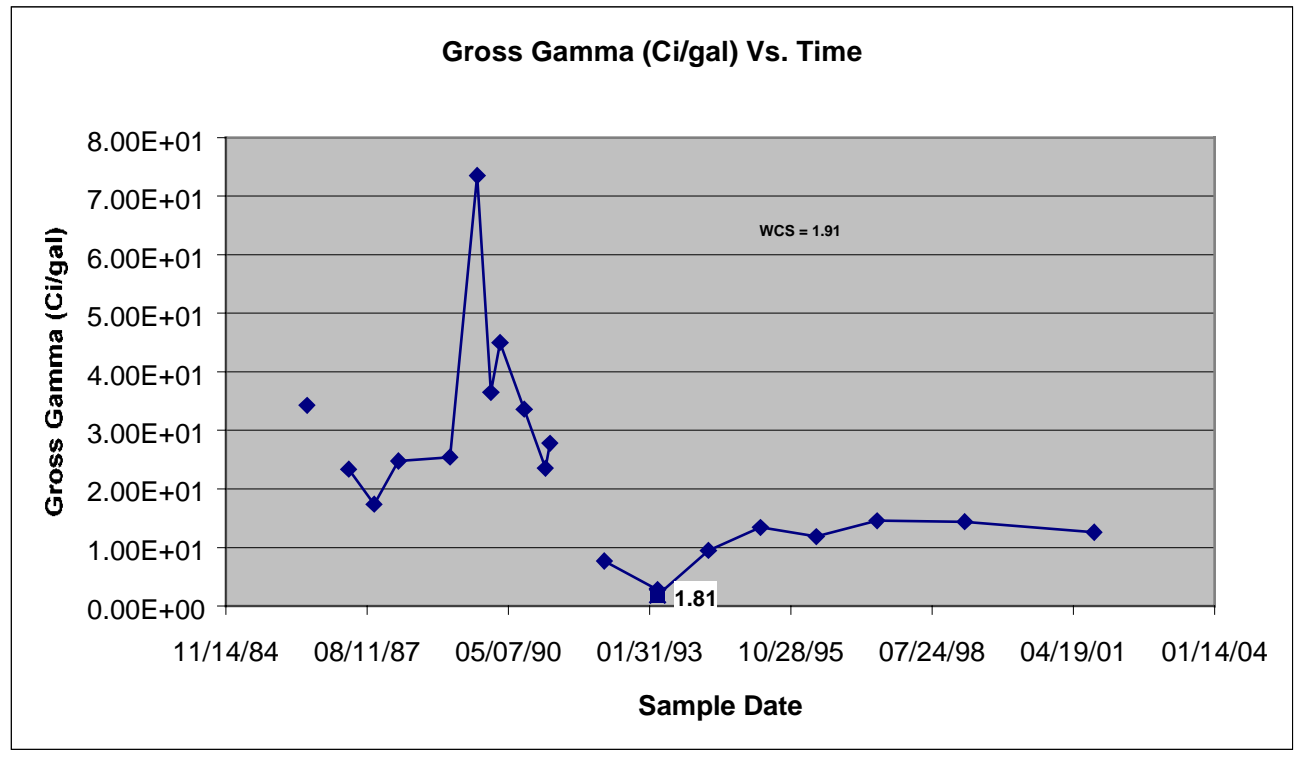


Tank 38

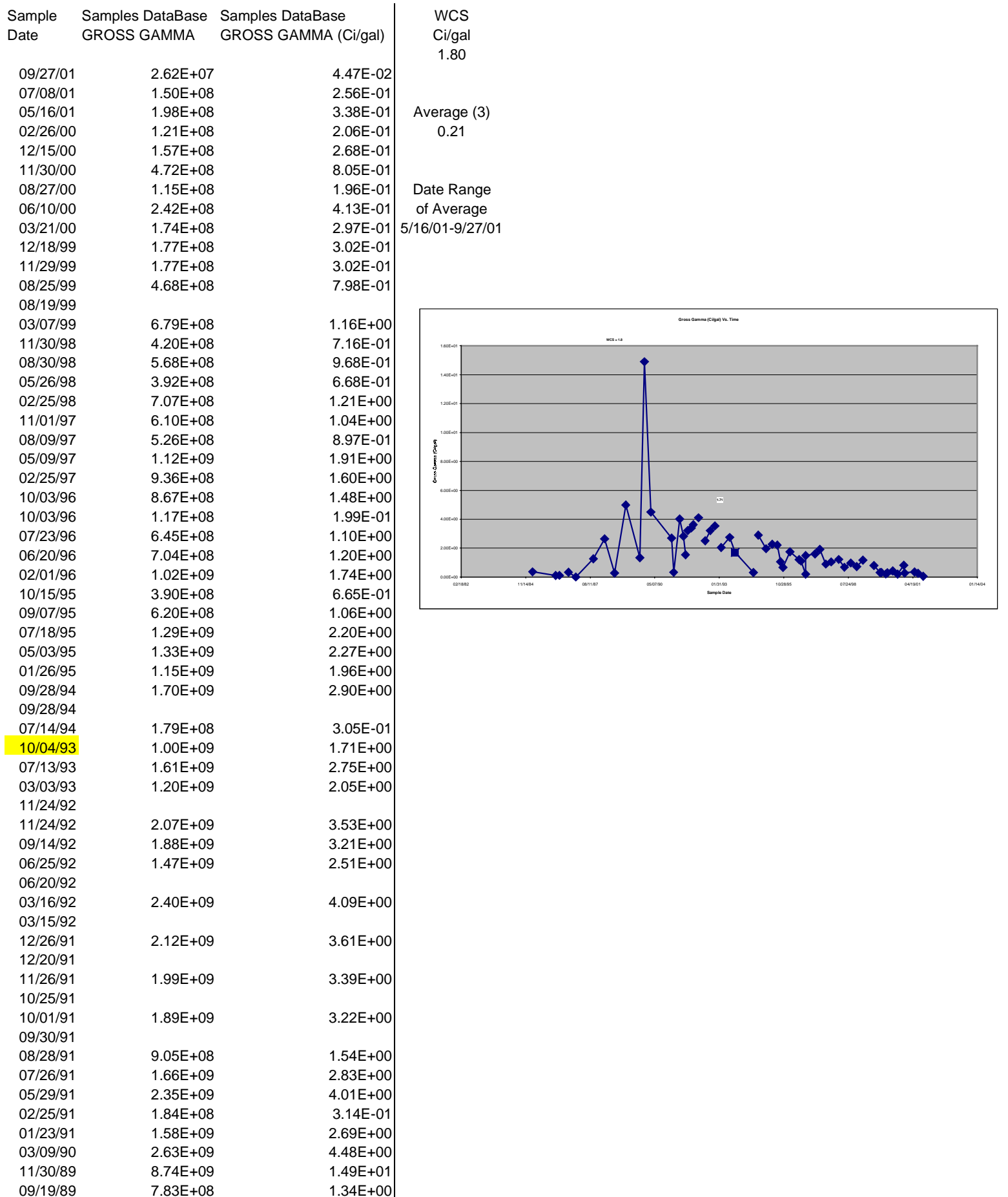


WSRC-TR-2001-00559, Rev. 1

Tank 41

\begin{tabular}{|c|c|c|c|c|c|}
\hline Sample & Samples DataBase & Samples DataBase & Samples Database & Samples Database & WCS \\
\hline Date & GROSS GAMMA & GROSS GAMMA (Ci/gal) & Cs-137 (d/m/ml) & Cs-137 (Ci/gal) & $\begin{array}{c}\mathrm{Ci} / \mathrm{gal} \\
3.01\end{array}$ \\
\hline $11 / 25 / 01$ & $1.22 \mathrm{E}+09$ & $2.08 \mathrm{E}+00$ & & & \\
\hline 06/19/01 & $1.40 \mathrm{E}+09$ & $2.39 \mathrm{E}+00$ & & & \\
\hline $01 / 09 / 00$ & $1.18 \mathrm{E}+09$ & $2.01 E+00$ & & & Average (3) \\
\hline $08 / 13 / 00$ & $1.25 \mathrm{E}+09$ & $2.13 E+00$ & & & 2.16 \\
\hline $02 / 17 / 00$ & $1.33 E+09$ & $2.27 E+00$ & & & \\
\hline 08/18/99 & $1.18 \mathrm{E}+09$ & $2.01 E+00$ & & & \\
\hline $02 / 18 / 99$ & $1.41 \mathrm{E}+09$ & $2.40 \mathrm{E}+00$ & & & Date Range \\
\hline 07/19/98 & $1.18 \mathrm{E}+09$ & $2.01 \mathrm{E}+00$ & & & of Average \\
\hline $01 / 19 / 98$ & $1.37 \mathrm{E}+09$ & $2.34 \mathrm{E}+00$ & & & $1 / 9 / 00-11 / 25 / 01$ \\
\hline $07 / 15 / 97$ & $1.23 E+09$ & $2.10 \mathrm{E}+00$ & & & \\
\hline $01 / 01 / 97$ & $1.06 \mathrm{E}+09$ & $1.81 \mathrm{E}+00$ & & & \\
\hline $07 / 26 / 96$ & & & & & \\
\hline $01 / 04 / 96$ & $2.01 \mathrm{E}+09$ & $3.43 \mathrm{E}+00$ & & & \\
\hline 07/06/95 & $1.78 \mathrm{E}+09$ & $3.04 \mathrm{E}+00$ & & & \\
\hline $02 / 26 / 95$ & $1.98 \mathrm{E}+09$ & $3.38 \mathrm{E}+00$ & & & \\
\hline 08/19/94 & $2.13 E+09$ & $3.63 E+00$ & & & \\
\hline $05 / 27 / 93$ & & & & & \\
\hline $10 / 27 / 92$ & $1.67 \mathrm{E}+09$ & $2.85 \mathrm{E}+00$ & $1.67 \mathrm{E}+09$ & $2.85 \mathrm{E}+00$ & \\
\hline $02 / 17 / 92$ & & & $1.86 \mathrm{E}+09$ & $3.17 E+00$ & \\
\hline $08 / 24 / 91$ & & & $5.38 \mathrm{E}+08$ & 9.17E-01 & \\
\hline $01 / 26 / 91$ & $2.30 \mathrm{E}+09$ & $3.92 \mathrm{E}+00$ & & & \\
\hline $03 / 01 / 90$ & $3.04 \mathrm{E}+09$ & $5.18 \mathrm{E}+00$ & & & \\
\hline $02 / 21 / 90$ & $3.04 \mathrm{E}+09$ & $5.18 \mathrm{E}+00$ & & & \\
\hline $08 / 23 / 89$ & $2.65 E+09$ & $4.52 E+00$ & & & \\
\hline $03 / 08 / 89$ & $1.65 \mathrm{E}+09$ & $2.81 \mathrm{E}+00$ & & & \\
\hline $04 / 01 / 87$ & $2.08 \mathrm{E}+09$ & $3.55 \mathrm{E}+00$ & $2.78 \mathrm{E}+08$ & $4.74 \mathrm{E}-01$ & \\
\hline $03 / 23 / 87$ & & & & & \\
\hline $03 / 20 / 87$ & & & & & \\
\hline 09/09/86 & $1.94 \mathrm{E}+09$ & $3.31 \mathrm{E}+00$ & $2.33 \mathrm{E}+08$ & 3.97E-01 & \\
\hline $04 / 21 / 86$ & $4.55 E+08$ & 7.76E-01 & & & \\
\hline 07/09/85 & & & & & \\
\hline $08 / 13 / 84$ & & & & & \\
\hline $02 / 26 / 84$ & & & & & \\
\hline $08 / 09 / 83$ & & & & & \\
\hline $05 / 17 / 83$ & & & & & \\
\hline $05 / 17 / 83$ & & & & & \\
\hline $04 / 22 / 83$ & & & & & \\
\hline $04 / 22 / 83$ & & & & & \\
\hline $04 / 22 / 83$ & & & & & \\
\hline $03 / 09 / 82$ & & & & & \\
\hline
\end{tabular}

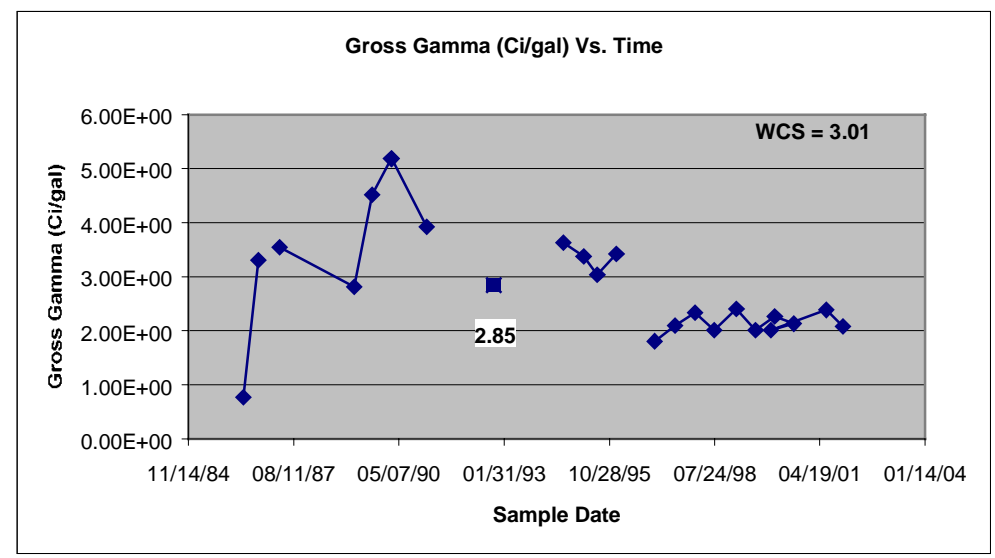


Tank 44

\begin{tabular}{|c|c|c|c|c|c|}
\hline Sample & Samples DataBase & Samples DataBase & Samples Database & Samples Database & WCS \\
\hline Date & GROSS GAMMA & GROSS GAMMA (Ci/gal) & Cs-137 (d/m/ml) & Cs-137 (Ci/gal) & $\begin{array}{l}\mathrm{Ci} / \mathrm{gal} \\
0.598\end{array}$ \\
\hline 02/03/99 & $2.94 \mathrm{E}+09$ & $5.01 \mathrm{E}+00$ & & & \\
\hline 05/01/97 & $3.58 \mathrm{E}+09$ & $6.10 \mathrm{E}+00$ & & & \\
\hline 04/23/96 & $3.21 E+09$ & $5.47 \mathrm{E}+00$ & & & Average (3) \\
\hline 04/27/95 & $3.22 \mathrm{E}+08$ & $5.49 \mathrm{E}-01$ & & & 5.53 \\
\hline 04/18/94 & $2.59 \mathrm{E}+09$ & $4.42 \mathrm{E}+00$ & $2.59 \mathrm{E}+09$ & $4.42 \mathrm{E}+00$ & \\
\hline 04/12/93 & $3.32 \mathrm{E}+08$ & $5.66 \mathrm{E}-01$ & $3.32 \mathrm{E}+08$ & $5.66 \mathrm{E}-01$ & \\
\hline 04/06/92 & $4.34 \mathrm{E}+09$ & $7.40 \mathrm{E}+00$ & $4.34 \mathrm{E}+09$ & $7.40 \mathrm{E}+00$ & Date Range \\
\hline $10 / 30 / 91$ & $3.12 \mathrm{E}+09$ & $5.32 \mathrm{E}+00$ & $3.12 \mathrm{E}+09$ & $5.32 \mathrm{E}+00$ & of Average \\
\hline 04/12/91 & $4.21 E+09$ & $7.18 \mathrm{E}+00$ & & & 4/23/96-2/3/99 \\
\hline $10 / 04 / 90$ & $6.54 \mathrm{E}+09$ & $1.12 \mathrm{E}+01$ & & & \\
\hline 08/08/90 & $6.70 \mathrm{E}+09$ & $1.14 \mathrm{E}+01$ & & & \\
\hline 03/21/90 & $2.66 \mathrm{E}+09$ & $4.54 \mathrm{E}+00$ & & & \\
\hline 08/15/89 & $4.70 \mathrm{E}+09$ & $8.01 E+00$ & & & \\
\hline 05/15/89 & $3.30 \mathrm{E}+09$ & $5.63 \mathrm{E}+00$ & & & \\
\hline $11 / 04 / 88$ & $2.33 \mathrm{E}+09$ & $3.97 \mathrm{E}+00$ & & & \\
\hline 05/18/88 & $4.50 \mathrm{E}+09$ & $7.67 \mathrm{E}+00$ & $5.69 \mathrm{E}+08$ & $9.70 \mathrm{E}-01$ & \\
\hline $04 / 21 / 88$ & $4.50 \mathrm{E}+09$ & $7.67 \mathrm{E}+00$ & & & \\
\hline \multicolumn{6}{|l|}{$08 / 06 / 87$} \\
\hline 04/03/87 & $1.14 \mathrm{E}+09$ & $1.94 \mathrm{E}+00$ & $1.69 \mathrm{E}+08$ & $2.88 \mathrm{E}-01$ & \\
\hline $11 / 25 / 86$ & $2.20 \mathrm{E}+09$ & $3.75 \mathrm{E}+00$ & $3.02 \mathrm{E}+08$ & $5.15 \mathrm{E}-01$ & \\
\hline \multicolumn{6}{|l|}{$04 / 26 / 85$} \\
\hline $01 / 22 / 85$ & $3.19 \mathrm{E}+09$ & $5.44 \mathrm{E}+00$ & $4.23 \mathrm{E}+08$ & 7.21E-01 & \\
\hline \multicolumn{6}{|l|}{ 02/09/84 } \\
\hline \multicolumn{6}{|l|}{ 08/23/83 } \\
\hline \multicolumn{6}{|l|}{$03 / 18 / 83$} \\
\hline 03/25/82 & & & & & \\
\hline
\end{tabular}

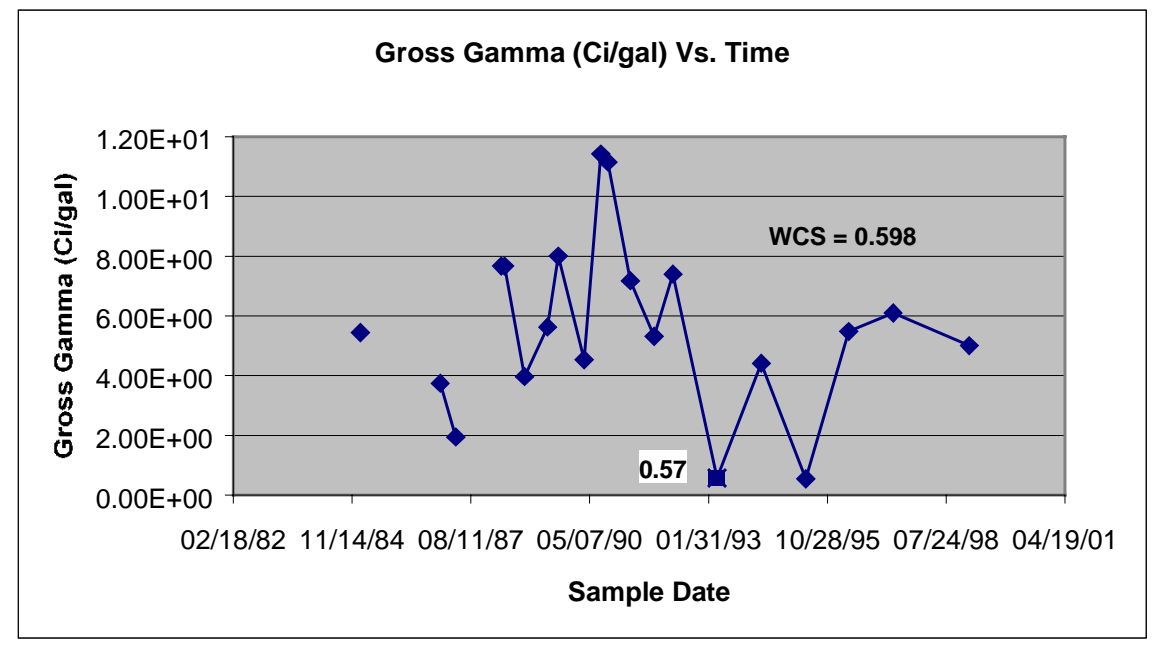


WSRC-TR-2001-00559, Rev. 1

Tank 45

\begin{tabular}{|c|c|c|c|c|c|}
\hline Sample & Samples DataBase & Samples DataBase & Samples Database & Samples Database & WCS \\
\hline Date & GROSS GAMMA & GROSS GAMMA (Ci/gal) & Cs-137 (d/m/ml) & Cs-137 (Ci/gal) & $\begin{array}{c}\mathrm{Ci} / \mathrm{gal} \\
3.82\end{array}$ \\
\hline $04 / 12 / 99$ & $2.78 E+09$ & $4.74 \mathrm{E}+00$ & & & \\
\hline 05/01/97 & $3.75 E+09$ & $6.39 \mathrm{E}+00$ & & & \\
\hline $04 / 10 / 96$ & $4.00 E+09$ & $6.82 \mathrm{E}+00$ & & & Average (3) \\
\hline 03/28/95 & $3.10 \mathrm{E}+09$ & $5.29 \mathrm{E}+00$ & & & 5.98 \\
\hline $02 / 12 / 94$ & $3.50 \mathrm{E}+09$ & $5.97 \mathrm{E}+00$ & $3.50 E+09$ & $5.97 \mathrm{E}+00$ & \\
\hline $02 / 24 / 93$ & $2.12 \mathrm{E}+09$ & $3.61 \mathrm{E}+00$ & $2.12 E+09$ & $3.61 \mathrm{E}+00$ & \\
\hline $11 / 13 / 92$ & $2.74 \mathrm{E}+09$ & $4.67 \mathrm{E}+00$ & & & Date Range \\
\hline $08 / 14 / 92$ & $3.65 \mathrm{E}+09$ & $6.22 \mathrm{E}+00$ & $3.65 E+09$ & $6.22 \mathrm{E}+00$ & of Average \\
\hline 05/19/92 & $3.43 E+09$ & $5.85 E+00$ & $3.43 E+09$ & $5.85 \mathrm{E}+00$ & 4/10/96-4/12/99 \\
\hline 05/19/92 & & & $7.40 \mathrm{E}+09$ & $1.26 \mathrm{E}+01$ & \\
\hline $02 / 26 / 92$ & $3.75 \mathrm{E}+09$ & $6.39 \mathrm{E}+00$ & $3.75 E+09$ & $6.39 E+00$ & \\
\hline 02/26/92 & & & $2.50 E+09$ & $4.26 \mathrm{E}+00$ & \\
\hline $01 / 17 / 92$ & $4.40 \mathrm{E}+09$ & $7.50 \mathrm{E}+00$ & $4.40 \mathrm{E}+09$ & $7.50 \mathrm{E}+00$ & \\
\hline $11 / 13 / 91$ & $3.95 E+09$ & $6.74 \mathrm{E}+00$ & $3.95 \mathrm{E}+09$ & $6.74 \mathrm{E}+00$ & \\
\hline $10 / 30 / 91$ & $3.44 \mathrm{E}+09$ & $5.87 \mathrm{E}+00$ & $3.44 \mathrm{E}+09$ & $5.87 \mathrm{E}+00$ & \\
\hline $09 / 15 / 91$ & $4.14 \mathrm{E}+09$ & $7.06 \mathrm{E}+00$ & $4.14 \mathrm{E}+09$ & $7.06 \mathrm{E}+00$ & \\
\hline 08/09/91 & $4.52 \mathrm{E}+09$ & $7.71 \mathrm{E}+00$ & & & \\
\hline $07 / 01 / 91$ & $1.56 E+09$ & $2.66 \mathrm{E}+00$ & & & \\
\hline $05 / 24 / 91$ & $2.60 E+09$ & $4.43 E+00$ & & & \\
\hline $04 / 12 / 91$ & $2.80 E+09$ & $4.77 \mathrm{E}+00$ & & & \\
\hline $10 / 12 / 90$ & $4.72 E+09$ & $8.05 E+00$ & & & \\
\hline $03 / 21 / 90$ & $4.91 E+09$ & $8.37 \mathrm{E}+00$ & & & \\
\hline $12 / 12 / 89$ & $6.96 \mathrm{E}+09$ & $1.19 \mathrm{E}+01$ & & & \\
\hline $11 / 17 / 89$ & $6.96 \mathrm{E}+09$ & 1.19E+01 & & & \\
\hline 05/15/89 & $1.96 \mathrm{E}+09$ & $3.34 \mathrm{E}+00$ & & & \\
\hline $11 / 04 / 88$ & $3.74 \mathrm{E}+09$ & $6.38 \mathrm{E}+00$ & & & \\
\hline 05/18/88 & $1.47 E+09$ & $2.51 E+00$ & $1.87 E+08$ & $3.19 \mathrm{E}-01$ & \\
\hline $04 / 21 / 88$ & $1.47 \mathrm{E}+09$ & $2.51 E+00$ & & & \\
\hline $10 / 07 / 87$ & $2.23 E+09$ & $3.80 \mathrm{E}+00$ & $3.72 \mathrm{E}+08$ & $6.34 \mathrm{E}-01$ & \\
\hline $04 / 03 / 87$ & $2.73 E+09$ & $4.65 E+00$ & $4.59 E+08$ & 7.83E-01 & \\
\hline $11 / 25 / 86$ & $4.80 \mathrm{E}+09$ & $8.18 \mathrm{E}+00$ & $6.66 \mathrm{E}+08$ & $1.14 \mathrm{E}+00$ & \\
\hline $04 / 04 / 86$ & $5.71 E+09$ & $9.74 \mathrm{E}+00$ & $8.50 E+08$ & $1.45 \mathrm{E}+00$ & \\
\hline $04 / 26 / 85$ & & & & & \\
\hline 02/09/84 & & & & & \\
\hline $11 / 04 / 83$ & & & & & \\
\hline $08 / 23 / 83$ & & & & & \\
\hline $03 / 18 / 83$ & & & & & \\
\hline
\end{tabular}

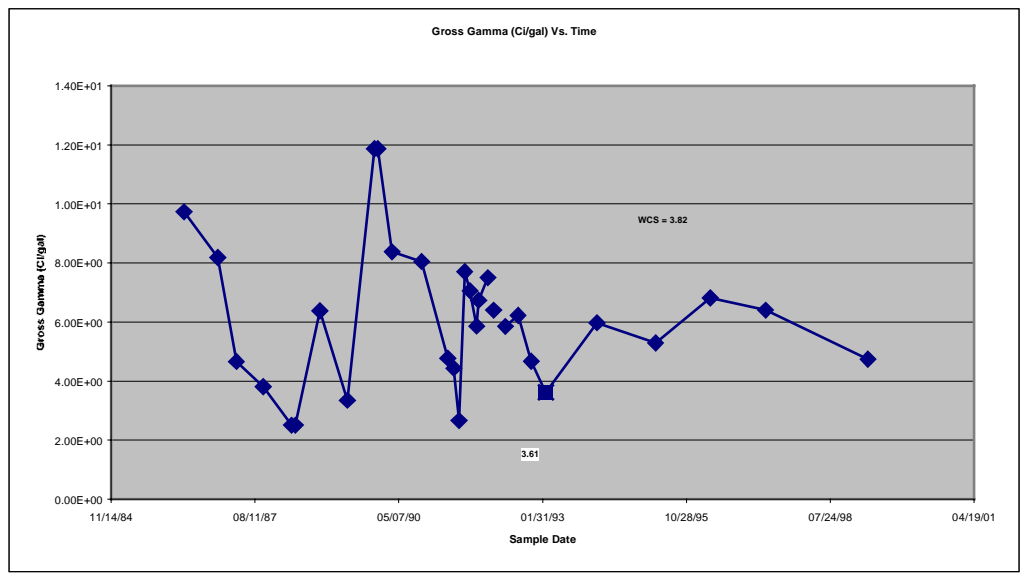


Tank 46

\begin{tabular}{|c|c|c|c|c|c|}
\hline $\begin{array}{l}\text { Sample } \\
\text { Date }\end{array}$ & $\begin{array}{l}\text { Samples DataBase } \\
\text { GROSS GAMMA }\end{array}$ & $\begin{array}{l}\text { Samples DataBase } \\
\text { GROSS GAMMA (Ci/aal) }\end{array}$ & $\begin{array}{l}\text { Samples Database } \\
\text { Cs-137 (d/m/ml) }\end{array}$ & $\begin{array}{l}\text { Samples Database } \\
\text { Cs-137 (Ci/gal) }\end{array}$ & $\begin{array}{l}\text { WCS } \\
\text { Ci/gal }\end{array}$ \\
\hline $11 / 01 / 01$ & $3.95 E+09$ & $6.74 \mathrm{E}+00$ & & & -- \\
\hline $08 / 15 / 01$ & $7.93 \mathrm{E}+08$ & $1.35 \mathrm{E}+00$ & & & \\
\hline 05/23/01 & $5.37 E+09$ & $9.16 \mathrm{E}+00$ & & & Average (3) \\
\hline 02/23/01 & 4.77E+09 & $8.13 E+00$ & & & 5.75 \\
\hline $12 / 05 / 00$ & $5.55 E+09$ & $9.46 \mathrm{E}+00$ & & & \\
\hline 09/07/00 & $5.94 \mathrm{E}+09$ & $1.01 \mathrm{E}+01$ & & & \\
\hline 06/30/00 & $5.91 E+09$ & $1.01 \mathrm{E}+01$ & & & Date Range \\
\hline 03/29/00 & $4.53 \mathrm{E}+09$ & $7.72 \mathrm{E}+00$ & & & of Average \\
\hline $12 / 08 / 99$ & $5.00 \mathrm{E}+08$ & 8.53E-01 & & & $5 / 23 / 01-11 / 01 / 01$ \\
\hline 05/20/99 & $3.55 E+09$ & $6.05 E+00$ & & & \\
\hline $12 / 07 / 98$ & $4.55 E+09$ & 7.76E +00 & $7.77 \mathrm{Ci} / \mathrm{gal}$ & 7.77 & \\
\hline 09/30/98 & $3.51 E+09$ & $5.98 \mathrm{E}+00$ & & & \\
\hline $04 / 21 / 98$ & $3.54 \mathrm{E}+09$ & $6.04 \mathrm{E}+00$ & & & \\
\hline $02 / 18 / 98$ & $2.89 \mathrm{E}+09$ & $4.93 \mathrm{E}+00$ & & & \\
\hline $11 / 14 / 97$ & $3.86 \mathrm{E}+09$ & $6.58 \mathrm{E}+00$ & & & \\
\hline $07 / 30 / 97$ & $3.23 E+09$ & $5.51 \mathrm{E}+00$ & & & \\
\hline 04/17/97 & $3.26 E+09$ & $5.56 \mathrm{E}+00$ & & & \\
\hline $01 / 13 / 97$ & $2.65 E+09$ & $4.52 \mathrm{E}+00$ & & & \\
\hline 08/01/96 & $2.55 E+09$ & $4.35 E+00$ & & & \\
\hline $04 / 25 / 96$ & $2.62 E+09$ & $4.47 \mathrm{E}+00$ & & & \\
\hline $11 / 21 / 95$ & $2.20 \mathrm{E}+09$ & $3.75 \mathrm{E}+00$ & & & \\
\hline 08/18/95 & $2.31 \mathrm{E}+09$ & $3.94 \mathrm{E}+00$ & & & \\
\hline $05 / 11 / 95$ & $1.96 \mathrm{E}+09$ & $3.34 \mathrm{E}+00$ & & & \\
\hline $03 / 06 / 95$ & $2.19 E+09$ & $3.73 \mathrm{E}+00$ & & & \\
\hline $11 / 23 / 94$ & $2.05 E+09$ & $3.50 \mathrm{E}+00$ & & & \\
\hline 08/20/94 & $2.42 E+09$ & $4.13 E+00$ & & & \\
\hline 05/13/94 & $2.36 \mathrm{E}+09$ & $4.02 E+00$ & 2.36E+09 & 4.02 & \\
\hline
\end{tabular}

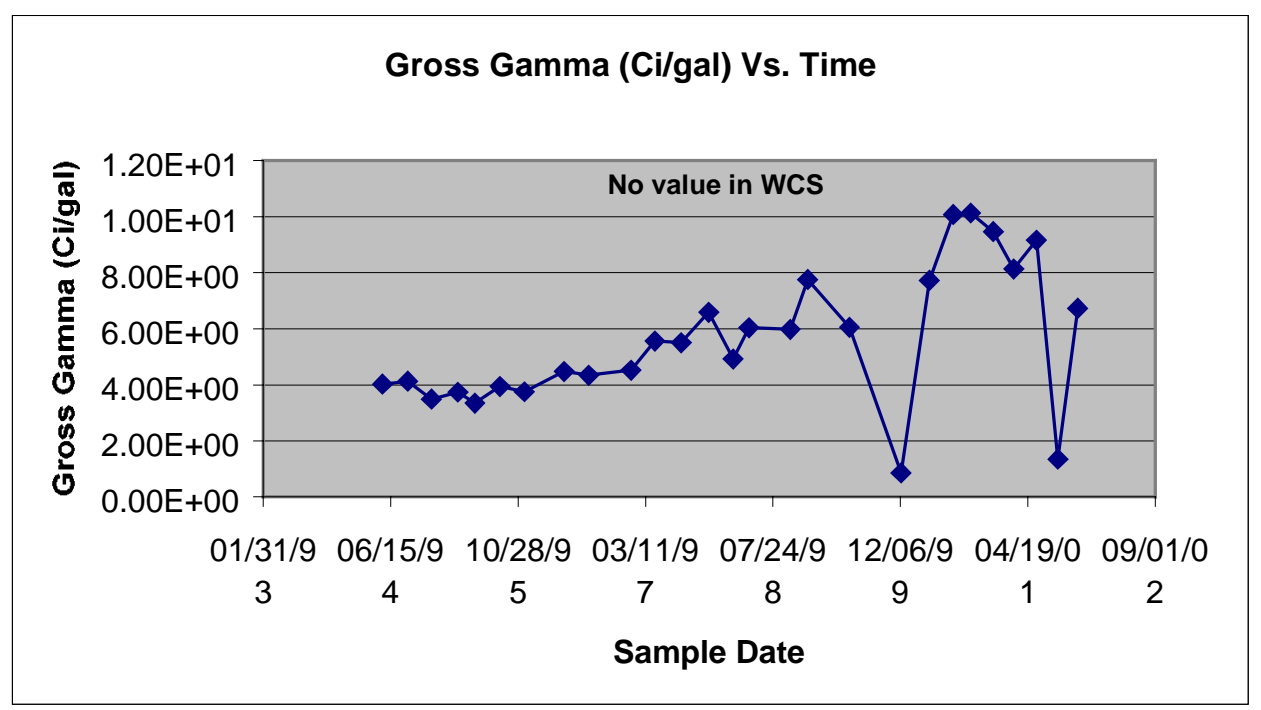


WSRC-TR-2001-00559, Rev. 1

Tank 47

\begin{tabular}{|c|c|c|c|c|c|}
\hline $\begin{array}{l}\text { Sample } \\
\text { Date }\end{array}$ & $\begin{array}{l}\text { Samples DataBase } \\
\text { GROSS GAMMA }\end{array}$ & $\begin{array}{l}\text { Samples DataBase } \\
\text { GROSS GAMMA (Ci/gal) }\end{array}$ & $\begin{array}{l}\text { Samples Database } \\
\text { Cs-137 (d/m/ml) }\end{array}$ & $\begin{array}{l}\text { Samples Database } \\
\text { Cs-137 (Ci/gal) }\end{array}$ & $\begin{array}{c}\text { WCS } \\
\mathrm{Ci} / \mathrm{gal} \\
4.16\end{array}$ \\
\hline $01 / 13 / 98$ & $1.48 \mathrm{E}+09$ & $2.52 \mathrm{E}+00$ & & & \\
\hline 01/13/97 & $1.50 \mathrm{E}+09$ & $2.56 \mathrm{E}+00$ & & & \\
\hline 05/22/96 & $1.72 E+09$ & $2.93 E+00$ & & & Average (3) \\
\hline 10/19/95 & $1.38 \mathrm{E}+09$ & $2.35 E+00$ & & & 2.67 \\
\hline 07/18/95 & $1.39 E+09$ & $2.37 \mathrm{E}+00$ & & & \\
\hline $04 / 27 / 95$ & $1.66 \mathrm{E}+08$ & 2.83E-01 & & & \\
\hline $01 / 20 / 95$ & $1.74 \mathrm{E}+09$ & $2.97 \mathrm{E}+00$ & & & Date Range \\
\hline $10 / 06 / 94$ & $1.76 \mathrm{E}+09$ & $3.00 \mathrm{E}+00$ & & & of Average \\
\hline 11/13/91 & $2.31 \mathrm{E}+09$ & $3.94 E+00$ & $2.31 \mathrm{E}+09$ & $3.94 \mathrm{E}+00$ & 5/22/96-1/13/98 \\
\hline 05/24/91 & $3.70 \mathrm{E}+09$ & $6.31 \mathrm{E}+00$ & & & \\
\hline $12 / 06 / 90$ & $5.42 \mathrm{E}+09$ & $9.24 \mathrm{E}+00$ & & & \\
\hline $05 / 24 / 90$ & $5.95 \mathrm{E}+09$ & $1.01 \mathrm{E}+01$ & & & \\
\hline $01 / 24 / 90$ & $4.81 \mathrm{E}+09$ & $8.20 E+00$ & & & \\
\hline $05 / 15 / 89$ & $5.12 \mathrm{E}+09$ & $8.73 E+00$ & & & \\
\hline $11 / 04 / 88$ & & & & & \\
\hline $04 / 21 / 88$ & $3.67 \mathrm{E}+09$ & $6.26 \mathrm{E}+00$ & $4.13 \mathrm{E}+08$ & 7.04E-01 & \\
\hline $11 / 25 / 86$ & $4.59 \mathrm{E}+00$ & 7.83E-09 & $6.40 \mathrm{E}+08$ & $1.09 \mathrm{E}+00$ & \\
\hline $04 / 04 / 86$ & $3.31 \mathrm{E}+09$ & $5.64 \mathrm{E}+00$ & $4.58 \mathrm{E}+08$ & 7.81E-01 & \\
\hline 08/19/85 & $2.57 \mathrm{E}+09$ & $4.38 \mathrm{E}+00$ & & & \\
\hline $04 / 09 / 85$ & $8.82 E+08$ & $1.50 \mathrm{E}+00$ & $1.08 \mathrm{E}+08$ & 1.84E-01 & \\
\hline 03/19/85 & & & & & \\
\hline 10/20/84 & & & & & \\
\hline $07 / 12 / 83$ & & & & & \\
\hline $01 / 14 / 83$ & & & & & \\
\hline 07/22/82 & & & & & \\
\hline 05/09/82 & & & & & \\
\hline $01 / 08 / 82$ & & & & & \\
\hline $10 / 16 / 81$ & & & & & \\
\hline $07 / 02 / 81$ & & & & & \\
\hline $07 / 02 / 81$ & & & & & \\
\hline $07 / 0$ & & & & & \\
\hline
\end{tabular}

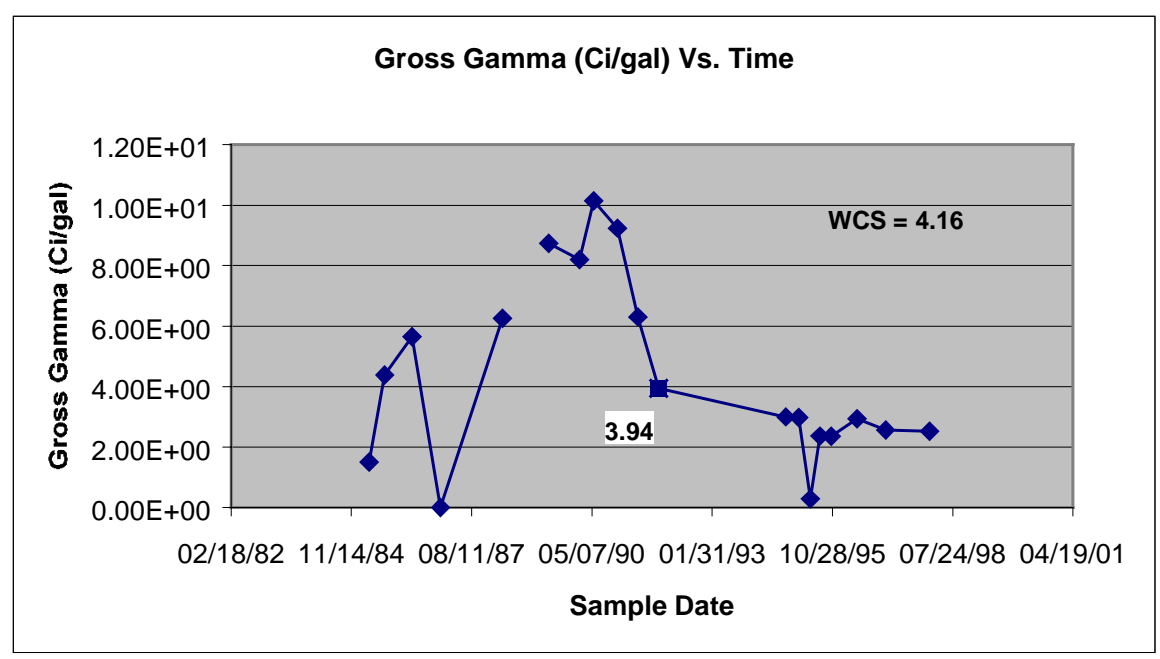

